\title{
THE L-HOMOLOGY FUNDAMENTAL CLASS FOR IP-SPACES AND THE STRATIFIED NOVIKOV CONJECTURE
}

\author{
MARKUS BANAGL, GERD LAURES, AND JAMES E. MCCLURE
}

\begin{abstract}
An IP-space is a pseudomanifold whose defining local properties imply that its middle perversity global intersection homology groups satisfy Poincaré duality integrally. We show that the symmetric signature induces a map of Quinn spectra from IP bordism to the symmetric $L$-spectrum of $\mathbb{Z}$, which is, up to weak equivalence, an $E_{\infty}$ ring map. Using this map, we construct a fundamental $L$-homology class for IP-spaces, and as a consequence we prove the stratified Novikov conjecture for IP-spaces whose fundamental group satisfies the Novikov conjecture.
\end{abstract}

\section{INTRODUCTION}

An intersection homology Poincaré space, or IP-space, is a piecewise linear pseudomanifold such that the middle dimensional, lower middle perversity integral intersection homology of even-dimensional links vanishes and the lower middle dimensional, lower middle perversity intersection homology of odd-dimensional links is torsion free. This class of spaces was introduced by Goresky and Siegel in GS83 as a natural solution, assuming the IP-space to be compact and oriented, to the question: For which class of spaces does intersection homology (with middle perversity) satisfy Poincaré duality over the integers?

If $X$ is a compact oriented IP-space whose dimension $n$ is a multiple of 4 , then the signature $\sigma(X)$ of $X$ is the signature of the intersection form

$$
I H_{n / 2}(X ; \mathbb{Z}) / \text { Tors } \times I H_{n / 2}(X ; \mathbb{Z}) / \text { Tors } \longrightarrow \mathbb{Z}
$$

where $I H_{*}$ denotes intersection homology with the lower middle perversity, GM80, GM83. This signature is a bordism invariant for bordisms of IPspaces. The IP-space bordism groups have been investigated by Pardon in [Par90], where it is shown that the signature (when $n=4 k$ ) together with

2010 Mathematics Subject Classification. 55N33, 57R67, 57R20, 57N80, 19G24.

Key words and phrases. Intersection homology, stratified spaces, pseudomanifolds, signature, characteristic classes, bordism, L-theory, Novikov conjecture.

The first author was supported in part by a research grant of the Deutsche Forschungsgemeinschaft. The third author was partially supported by a grant from the Simons Foundation (\#279092 to James McClure). He thanks the Lord for making his work possible. 
the de Rham invariant (when $n=4 k+1$ ) form a complete system of invariants.

Next we recall the theory of the $L$-homology fundamental class for manifolds. Let $M^{n}$ be a closed oriented $n$-dimensional manifold. The symmetric signature $\sigma^{*}(M)$ of [M71, Ran92, p. 47] is an element of the symmetric $L$ group $L^{n}(\mathbb{Z}[G])$, where $G$ denotes the fundamental group $\pi_{1} M$. It is a nonsimply-connected generalization of the signature $\sigma(M)$, since for $n=4 k$ the canonical homomorphism $L^{n}(\mathbb{Z}[G]) \rightarrow L^{n}(\mathbb{Z})=\mathbb{Z}$ maps $\sigma^{*}(M)$ to $\sigma(M)$. Moreover, $\sigma^{*}$ is homotopy invariant and bordism invariant for bordisms over the classifying space $B G$. Let $\mathbb{L} \bullet=\mathbb{L} \bullet\langle 0\rangle(\mathbb{Z})$ denote the symmetric $L$-spectrum with homotopy groups $\pi_{n}\left(\mathbb{L}^{\bullet}\right)=L^{n}(\mathbb{Z})$ and let $\mathrm{L}_{*}^{\bullet}(-)$ denote the homology theory determined by $\mathbb{L}^{\bullet}$. For an $n$-dimensional Poincaré space $M$ which is either a topological manifold or a combinatorial homology manifold (i.e. a polyhedron whose links of simplices are homology spheres), Ranicki defines a canonical $\mathrm{L}^{\bullet}$-homology fundamental class $[M]_{\mathbb{L}} \in \mathrm{L}_{n}^{\bullet}(M)$, see [Ran92, Prop. 16.16]. Its image under the assembly map

$$
\mathrm{L}_{n}^{\bullet}(M) \stackrel{\alpha}{\longrightarrow} L^{n}(\mathbb{Z}[G])
$$

is the symmetric signature $\sigma^{*}(M)$. The class $[M]_{\mathbb{L}}$ is a topological invariant, but, unlike the symmetric signature, not a homotopy invariant in general. The geometric meaning of the $\mathrm{L}^{\bullet}$-homology fundamental class is that its existence for a geometric Poincaré complex $X^{n}, n \geq 5$, assembling to the symmetric signature (which in fact any Poincaré complex possesses), implies up to 2-torsion that $X$ is homotopy equivalent to a compact topological manifold. (More precisely, $X$ is homotopy equivalent to a compact manifold if it has an $\mathrm{L}^{\bullet}$-homology fundamental class, which assembles to the so-called visible symmetric signature of $X$.) Smooth manifolds $M$ possess a Hirzebruch $L$-class in $H^{*}(M ; \mathbb{Q})$, whose Poincaré dual we denote by $L(M) \in H_{*}(M ; \mathbb{Q})$. Rationally, $[M]_{\mathbb{L}}$ is then given by $L(M)$,

$$
[M]_{\mathbb{L}} \otimes 1=L(M) \in \mathrm{L}_{n}^{\bullet}(M) \otimes \mathbb{Q} \cong \bigoplus_{j \geq 0} H_{n-4 j}(M ; \mathbb{Q}) .
$$

Thus, we may view $[M]_{\mathbb{L}}$ as an integral refinement of the $L$-class of $M$. The identity $\alpha[M]_{\mathbb{L}}=\sigma^{*}(M)$ may then be interpreted as a non-simply connected generalization of the Hirzebruch signature formula. These facts show that the $\mathrm{L}^{\bullet}$-homology fundamental class is much more powerful than $\sigma^{*}(M)$. For example, there exist infinitely many manifolds $M_{i}, i=1,2, \ldots$, in the homotopy type of $S^{2} \times S^{4}$, distinguished by the first Pontrjagin class of their tangent bundle $p_{1}\left(T M_{i}\right) \in H^{4}\left(S^{2} \times S^{4}\right) \cong \mathbb{Z}$, namely $p_{1}\left(T M_{i}\right)=K i, K$ a fixed nonzero integer. On the other hand, $\sigma^{*}\left(M_{i}\right)=\sigma^{*}\left(S^{2} \times S^{4}\right)=0 \in$ $L^{6}\left(\mathbb{Z}\left[\pi_{1}\left(S^{2} \times S^{4}\right)\right]\right)=L^{6}(\mathbb{Z})=0$.

We return to singular spaces. A Witt space is a piecewise linear pseudomanifold such that the middle dimensional, lower middle perversity rational 
intersection homology of even-dimensional links vanishes, [Sie83. The symmetric signature $\sigma^{*}(X) \in L^{n}(\mathbb{Q}[G])$ and the $L^{\bullet}$-homology fundamental class $[X]_{\mathbb{L}} \in(\mathrm{L} \bullet(\mathbb{Q}))_{n}(X)$ of an oriented Witt space $X^{n}$ appeared first in the work of Cappell, Shaneson and Weinberger, see CSW91 and W94, though a detailed construction is not provided there. Regarding the relation between $\mathrm{L}^{\bullet}(\mathbb{Z})$ and $\mathrm{L}^{\bullet}(\mathbb{Q})$, the functor $\operatorname{ad}_{\mathrm{sym}}$, described in $[\mathrm{LM} 13$, from the category of rings with involution to the category of ad theories associates to the localization map $\mathbb{Z} \rightarrow \mathbb{Q}$ a morphism ad $\mathrm{d}^{\mathbb{Z}} \rightarrow \mathrm{ad}^{\mathbb{Q}}$ of the associated symmetric Poincaré ad theories, which in turn induces a map of Quinn spectra $\mathrm{L}^{\bullet}(\mathbb{Z}) \rightarrow \mathrm{L}^{\bullet}(\mathbb{Q})$. On homotopy groups, this fits into Ranicki's localization sequence

$$
\cdots \longrightarrow L^{n}(\mathbb{Z}) \longrightarrow L^{n}(\mathbb{Q}) \longrightarrow L^{n}(\mathbb{Z}, \mathbb{Z}-\{0\}) \longrightarrow L^{n-1}(\mathbb{Z}) \longrightarrow \cdots
$$

where

$$
L^{n}(\mathbb{Q}) \cong \begin{cases}\mathbb{Z} \oplus(\mathbb{Z} / 2)^{\infty} \oplus(\mathbb{Z} / 4)^{\infty}, & n \equiv 0(4) \\ 0, & n \neq 0(4) .\end{cases}
$$

In [Ban11, the first author outlined a construction of $[X]_{\mathbb{L}}$ for IP-spaces $X$ based on ideas of Eppelmann [Epp07], and pointed out that the existence of this class implies in particular a definition of a symmetric signature $\sigma^{*}(X)$ as the image of $[X]_{\mathbb{L}}$ under assembly. In [ALMP12], it is shown that this symmetric signature, adapted to Witt spaces and pushed into $K_{*}\left(C_{r}^{*} G\right)$ via

$$
L^{*}(\mathbb{Q}[G]) \longrightarrow L^{*}\left(C_{r}^{*} G\right) \longrightarrow K_{*}\left(C_{r}^{*} G\right)
$$

agrees rationally with the Albin-Leichtnam-Mazzeo-Piazza signature index class. The first fully detailed construction of $\sigma^{*}(X)$ for Witt spaces $X$ has been provided in FM13b. That construction is closely parallel to the original construction of Miščenko, but using singular intersection chains on the universal cover instead of ordinary chains. The methods of [FM13b] carry over to IP-spaces and yield a symmetric signature over $\mathbb{Z}$ for such spaces, as we show in Section 8 .

In the present paper, we give the first detailed construction of an $\mathrm{L}^{\bullet}$ homology fundamental class $[X]_{\mathbb{L}} \in \mathrm{L}_{n}^{\bullet}(X)$ for IP-spaces $X$. While Eppelmann used complexes of sheaves, we are able to use the, for our purposes, more precise and geometric methods of [FM13b]. The main issue is to construct a map (at least in the derived category) on the spectrum level from IP bordism to $\mathbb{L}^{\bullet}$, for then $[X]_{\mathbb{L}}$ can readily be defined as the image of the identity map $\left[\operatorname{id}_{X}\right] \in\left(\Omega_{\mathrm{IP}}\right)_{n}(X)$ under $\left(\Omega_{\mathrm{IP}}\right)_{n}(X) \rightarrow \mathrm{L}_{n}^{\bullet}(X)$, see Definition 9.6. To obtain this map of spectra, we rely heavily on the technology of ad theories and their associated Quinn spectra as developed by the second and third author in [LM13], [LM]. Roughly, we construct first an ad theory of IP spaces, which automatically gives an associated Quinn spectrum $\mathbf{Q}_{\mathrm{IP}}$, whose homotopy groups are Pardon's IP bordism groups. Using the symmetric signature, we define a morphism of ad theories from the IP ad theory to the ad theory of symmetric algebraic Poincaré complexes over $\mathbb{Z}$. The spectrum 
of the latter ad theory is the symmetric $L$-spectrum $\mathbb{L}^{\bullet}$. The morphism of ad theories then induces the desired map of spectra. We prove that our $\mathrm{L}^{\bullet}$ homology fundamental class has all the expected properties (Theorem 9.3): It is an oriented PL homeomorphism invariant, its image under assembly is the symmetric signature and it agrees with Ranicki's $L^{\bullet}$-homology fundamental class when $X$ is a PL manifold 1

As an application of our $\mathrm{L}^{\bullet}$-homology fundamental class, we discuss the stratified homotopy invariance of the higher signatures of IP-spaces. Let $X$ be an $n$-dimensional compact oriented IP-space, whose fundamental group $G$ satisfies the strong Novikov conjecture, that is, the assembly map

$$
\mathrm{L}_{n}^{\bullet}(B G) \longrightarrow L^{n}(\mathbb{Z}[G])
$$

is rationally injective. Then we prove that the stratified Novikov conjecture holds for $X$, i.e. the higher signatures

$$
\left\langle a, r_{*} L(X)\right\rangle, a \in H^{*}(B G ; \mathbb{Q}),
$$

where $r: X \rightarrow B G$ is a classifying map for the universal cover of $X$ and $L(X) \in H_{*}(X ; \mathbb{Q})$ is the Goresky-MacPherson $L$-class of $X$, are stratified homotopy invariants, see Theorem 11.2, The stratified Novikov conjecture has been treated from the analytic viewpoint in [ALMP13].

Here is an outline of the paper. Sections 2 and 3 review the basic facts about IP-spaces and ad theories. Section 4 constructs an ad theory associated to IP-spaces. Section 5 reviews two (equivalent) ad theories associated to symmetric Poincaré complexes. Section 6 uses the symmetric signature (ignoring the fundamental group) to construct a map Sig of Quinn spectra from IP bordism to the symmetric $L$-spectrum of $\mathbb{Z}$. Section 7 recalls the definition of $L^{n}\left(\mathbb{Z}\left[\pi_{1} X\right]\right)$, where $\pi_{1} X$ denotes the fundamental groupoid of $X$. Section 8 constructs the symmetric signature of an IP-space $X$ as an element of $L^{n}\left(\mathbb{Z}\left[\pi_{1} X\right]\right)$. Section 9 constructs the $\mathrm{L}^{\bullet}$-theory fundamental class of an IP-space, and Section 10 shows that it assembles to the symmetric signature constructed in Section 8. Section 11 uses the results of Sections 9 and 10 to prove the stratified Novikov conjecture for IP-spaces. Section 12 shows that the map Sig constructed in Section 6 is, up to weak equivalence, an $E_{\infty}$ ring map; this is applied in Section 13 to prove that $[X \times Y]_{\mathbb{L}}=[X]_{\mathbb{L}} \times[Y]_{\mathbb{L}}$. Section 14 proves a result needed for Section 9, namely the fact that the assembly map for IP bordism is a weak equivalence. There are six appendices. Appendix $\mathrm{A}$ reviews the basic facts about the intrinsic filtration of a PL space. Appendix B gives background about modules over additive categories which is needed for Sections 8 10. Appendices $\mathrm{C}$, D] and E give generalizations of some technical results from [FM13b] which are needed in

\footnotetext{
${ }^{1}$ If $R$ is a PID then our methods would also give a fundamental class in $\left(\mathrm{L}^{\bullet}(R)\right)_{n}(X)$ with analogous properties.
} 
Sections 8 and 10. Appendix $\mathrm{F}$ proves a mutliplicative property of the assembly map which may be of independent interest. Appendix $\mathrm{G}$ corrects some signs in LM13.

Remark 1.1. Here is a comparison between our notation and that in Ran92]. The spectrum $\mathbb{L}^{\bullet}\left(\mathbb{Z}\left[\pi_{1}(X, x)\right]\right)$ of [Ran92, Definition 14.1] is identical with the spectrum we denote by $\mathbf{Q}^{\mathbb{Z}\left[\pi_{1}(X, x)\right]}$ (which was defined in [LM13, Sections 9 and 15]). The spectrum $\mathbb{L} \bullet\langle 0\rangle\left(\mathbb{Z}\left[\pi_{1}(X, x)\right]\right)$ of [Ran92, pages 151-152] is canonically weakly equivalent to the spectrum we denote by

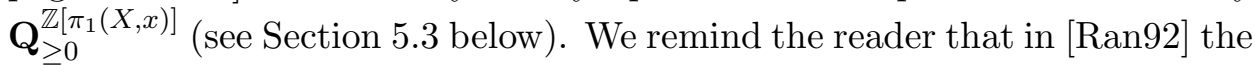
spectrum $\mathbb{L} \bullet\langle 0\rangle(\mathbb{Z})$ is denoted by $\mathbb{L}([\operatorname{Ran} 92$, page 173$])$ and $L^{n}\left(\mathbb{Z}\left[\pi_{1}(X, x)\right]\right)$ is defined to be $\pi_{n}$ of the connective spectrum $\mathbb{L} \bullet\langle 0\rangle\left(\mathbb{Z}\left[\pi_{1}(X, x)\right]\right)$ ([Ran92, page 60]).

Acknowledgements. We would like to thank Matthias Kreck, Wolfgang Lück, Shmuel Weinberger and (especially) Greg Friedman for their help. We are also grateful to the referee for a very careful revision.

\section{REVIEW OF IP BORDISM}

We use the term polyhedron as defined in [RS72, Definition 1.1].

Definition 2.1. An $n$-dimensional $P L$ pseudomanifold is a polyhedron $X$ for which some (and hence every) triangulation has the following properties.

(a) Every simplex is contained in an $n$-simplex.

(b) Every $(n-1)$-simplex is a face of exactly two $n$-simplices.

Definition 2.2. An $n$-dimensional $P L \partial$-pseudomanifold is a polyhedron $X$ with the property that some (and hence every) triangulation has the following properties.

(a) Every simplex is contained in an $n$-simplex.

(b) Every $(n-1)$-simplex is a face of either one or two $n$-simplices; the union of the $(n-1)$-simplices which are faces of one $n$-simplex is called the boundary of $X$ and denoted $\partial X$.

(c) The boundary $\partial X$ is an $(n-1)$-dimensional pseudomanifold.

(d) The boundary is collared, that is, there is a PL embedding $\partial X \times$ $[0,1) \rightarrow X$ with open image which is the identity on $\partial X$.

Remark 2.3. (i) The subspace $\partial X$ is independent of the triangulation.

(ii) The collaring condition is needed in order for Lefschetz duality to hold in intersection homology (see [FM13a, Section 7.3]).

Definition 2.4. An orientation of an $n$-dimensional PL pseudomanifold or PL $\partial$-pseudomanifold is a set of orientations of the $n$-simplices of some triangulation such that the sum of the $n$-simplices with these orientations is a cycle (a relative cycle in the case of a $\partial$-pseudomanifold). 
For some purposes we need a stratification. For a polyhedron $Y$, let $c^{\circ} Y$ denote the open cone $([0,1) \times Y) /(0 \times Y)$. We recall the inductive definition of stratified pseudomanifold:

Definition 2.5. A 0-dimensional stratified $P L$ pseudomanifold $X$ is a discrete set of points with the trivial filtration $X=X^{0} \supseteq X^{-1}=\emptyset$. An $n$ dimensional stratified $P L$ pseudomanifold $X$ is a polyhedron together with a filtration by closed polyhedra

$$
X=X^{n} \supseteq X^{n-1}=X^{n-2} \supseteq \cdots \supseteq X^{0} \supseteq X^{-1}=\emptyset
$$

such that

(a) $X-X^{n-1}$ is dense in $X$, and

(b) for each point $x \in X^{i}-X^{i-1}$, there exists a neighborhood $U$ of $x$ for which there is a compact $n-i-1$ dimensional stratified PL pseudomanifold $L$ and a PL homeomorphism

$$
\phi: \mathbb{R}^{i} \times c^{\circ} L \rightarrow U
$$

that takes $\mathbb{R}^{i} \times c^{\circ}\left(L^{j-1}\right)$ onto $X^{i+j} \cap U$.

The space $L$ in part (b) is determined up to PL homeomorphism by $x$ and the stratification ([F, Lemma 2.5.18]); it is called the link of $X$ at $x$ and denoted $L_{x}$. Since the cone on the empty set $L^{-1}$ is a point, taking $j=0$ in (b) shows that $X^{i}-X^{i-1}$ is a manifold for every $i$. A PL pseudomanifold always possesses a stratification in the sense of Definition 2.5. by Proposition A.1(iv). Conversely, if $X$ is an $n$-dimensional stratified PL pseudomanifold, then for any triangulation, every simplex is contained in an $n$-simplex and every $(n-1)$-simplex is a face of exactly two $n$-simplices, that is, the underlying polyhedron of $X$ is indeed a PL pseudomanifold in the sense of Definition 2.1.

Definition 2.6. An $n$-dimensional stratified $P L$-pseudomanifold is a PL $\partial$-pseudomanifold $X$ together with a filtration by closed polyhedra such that

(a) $X-\partial X$, with the induced filtration, is an $n$-dimensional stratified PL pseudomanifold,

(b) $\partial X$, with the induced filtration, is an $n-1$ dimensional stratified PL pseudomanifold, and

(c) there is a neighborhood $N$ of $\partial X$ with a homeomorphism of filtered spaces $N \rightarrow \partial X \times[0,1)$ (where $[0,1)$ is given the trivial filtration) which is the identity on $\partial X$.

A PL $\partial$-pseudomanifold always possesses a stratification in the sense of Definition 2.6, by Proposition A.2, Next recall the definition of intersection homology ([GM80], GM83], Bo84], [KW06], Ban07]). We will denote the lower middle perversity, as usual, by $\bar{m}$.

Definition 2.7. (GS83, Par90]) An $n$-dimensional IP-space is an $n$ dimensional PL pseudomanifold $X$ for which some stratification has the following properties. 
(a) $I H_{l}^{\bar{m}}\left(L_{x} ; \mathbb{Z}\right)=0$ for all $x \in X^{n-2 l-1}-X^{n-2 l-2}$, and

(b) $I H_{l-1}^{\bar{m}}\left(L_{x} ; \mathbb{Z}\right)$ is torsion free for all $x \in X^{n-2 l}-X^{n-2 l-1}$.

Remark 2.8. (i) IP stands for "intersection homology Poincaré".

(ii) Note that the stratification is not considered as part of the structure of an IP-space.

(iii) If conditions (a) and (b) hold for some stratification then they hold for every stratification (by the Proposition in [GM83, Section 2.4]).

Definition 2.9. ([Par90]) An $n$-dimensional $\partial$-IP-space is an $n$-dimensional PL $\partial$-pseudomanifold $X$ for which $X-\partial X$ is an IP-space.

Proposition 2.10. If $X$ is a $\partial$-IP-space then $\partial X$ is an IP-space.

Proof. Give $X$ the stratification of Proposition A.2. By Remark 2.8(iii), the restriction of this stratification to $X-\partial X$ has properties (a) and (b) of Definition 2.7. Part (c) of Definition 2.6 implies that the links of $\partial X$ are also links of $X-\partial X$, so $\partial X$ satisfies Definition 2.7.

Next we consider IP bordism groups. There are two ways to define them:

(1) The objects and bordisms are the compact oriented IP-spaces and $\partial$-IP-spaces.

(2) An object is a compact oriented IP-space with a given stratification, and similarly for the bordisms.

Pardon Par90 does not make it clear which definition he is using, but fortunately the two definitions give the same bordism groups by $\mathrm{Fa}$. We will use the first definition.

\section{REVIEW OF ad THEORIES}

We recall some definitions from LM13, Sections 2 and 3]. A category with involution is a category together with an endofunctor $i$ which satisfies $i^{2}=1$. The set of integers $\mathbb{Z}$ is a poset and therefore a category. We give it the trivial involution. A $\mathbb{Z}$-graded category is a category $\mathcal{A}$ with involution together with involution-preserving functors $d: \mathcal{A} \rightarrow \mathbb{Z}$ (called the dimension function) and $\varnothing: \mathbb{Z} \rightarrow \mathcal{A}$ such that $d \varnothing$ is equal to the identity functor. A $k$-morphism between $\mathbb{Z}$-graded categories is a functor which decreases the dimensions of objects by $k$ and strictly commutes with $\varnothing$ and $i$. We will write $\varnothing_{n}$ for $\varnothing(n)$. Note that the existence of $d$ implies that when $d(A)>d(B)$, there are no morphisms $A \rightarrow B$.

Let $K$ be a finite collection of PL balls in some $\mathbb{R}^{n}$, and write $|K|$ for the union $\bigcup_{\sigma \in K} \sigma$. We say that $K$ is a ball complex if the interiors of the balls of $K$ are disjoint and the boundary of each ball of $K$ is a union of balls of $K$ (thus the interiors of the balls of $K$ give $|K|$ the structure of a regular CW complex). The balls of $K$ will also be called closed cells of $K$. A subcomplex of a ball complex $K$ is a subset of $K$ which is a ball complex. A morphism of ball complexes is the composite of an isomorphism with an inclusion of a subcomplex. For a ball complex $K$ and a subcomplex $L$ we 
define $\mathcal{C}$ ell $(K, L)$ to be the category in which the objects are the oriented closed cells of $K$ which are not in $L$, together with an empty cell $\emptyset_{n}$ for each dimension $n$, and the non-identity morphisms are given by inclusions of cells (with no requirement on the orientations). The category $\mathcal{C} e l l(K, L)$ is $\mathbb{Z}$-graded ([LM13, Definition 3.3]), that is, it comes with an involution $i$ (which reverses the orientation), a dimension functor $d$ into the poset $\mathbb{Z}$ and a section functor given by $\emptyset_{n}$.

Given a $\mathbb{Z}$-graded category $\mathcal{A}$, a pre $(K, L)$-ad of degree $k$ is a functor $\mathcal{C}$ ell $(K, L) \rightarrow \mathcal{A}$ of $\mathbb{Z}$-graded categories which decreases dimensions by $k$. The set of these is denoted $\operatorname{pre}^{k}(K, L)$. Note that a pre $(K, L)$-ad defines a pre $K=(K, \emptyset)$-ad by precomposition with the functor from $\mathcal{C} e l l(K)$ to $\mathcal{C}$ ell $(K, L)$ which sends the $n$-dimensional cells of $L$ to the empty cell $\emptyset_{n}$.

An ad theory with values in $\mathcal{A}$ (called the target category of the ad theory) consists of a subset $\operatorname{ad}^{k}(K, L) \subset \operatorname{pre}^{k}(K, L)$ for each $(K, L)$ and each $k$, satisfying certain axioms ([LM13, Definition 3.10]). One of the axioms says that an element of $\operatorname{pre}^{k}(K, L)$ is in $\operatorname{ad}^{k}(K, L)$ if and only if its image in $\operatorname{pre}^{k}(K)$ is in $\operatorname{ad}^{k}(K)$, so to describe an ad theory it suffices to specify the sets $\operatorname{ad}^{k}(K)$.

An ad theory gives rise to bordism groups $\Omega_{*}$ ([LM13, Section 4]), a spectrum Q ([Q95, [LM13, Section 15]), and a weakly equivalent symmetric spectrum M ([LM13, Section 17]).

A morphism of ad theories is a functor of target categories which takes ads to ads. A morphism $\operatorname{ad}_{1} \rightarrow \operatorname{ad}_{2}$ of ad theories induces maps $\mathbf{Q}_{1} \rightarrow \mathbf{Q}_{2}$ and $\mathbf{M}_{1} \rightarrow \mathbf{M}_{2}$ of the associated spectra.

Remark 3.1. Later we will need to know that there is a canonical isomorphism $\pi_{j} \mathbf{Q} \cong \Omega_{j}$ for all $j$. This is a consequence of [LM13, Proposition 16.4(i), Remark 14.2(i), and Definitions 4.1 and 4.2]; for the convenience of the reader we describe the isomorphism more explicitly. By definition, $\pi_{j} \mathbf{Q}$ is $\operatorname{colim}_{n} \pi_{n+j} Q_{n}$, where the $Q_{n}$ are the spaces of the spectrum, and the maps $\pi_{n+j} Q_{n} \rightarrow \pi_{n+1+j} Q_{n+1}$ are induced by the suspension maps ([LM13, page 44] and Appendix G) and are isomorphisms because $\mathbf{Q}$ is an $\Omega$ spectrum ([LM13, Proposition 15.9]). It therefore suffices to give compatible maps

$$
\pi_{n+j} Q_{n} \rightarrow \Omega_{j}
$$

for all $n$. Next recall that $Q_{n}$ is the geometric realization of a based semisimplicial set $P_{n}$ ([LM13, Definition 15.4]). If $s$ is an $(n+j)$ simplex of $P_{n}$ which has all its faces at the basepoint then the induced map $|s / \partial s| \rightarrow Q_{n}$ represents an element $\bar{s}$ of $\pi_{n+j} Q_{n}$, and since $P_{n}$ is a Kan complex ([LM13, Lemma 15.12]) all elements of $\pi_{n+j} Q_{n}$ are obtained in this way ([RS71, Remark 6.5]). Also, by [LM13, Definitions 15.4 and 3.10(e)], there is an element $s_{0} \in \operatorname{ad}^{-j}(*)$ which corresponds to $s$ under the map induced by the $(n+j)$-isomorphism from $\mathcal{C} \operatorname{ell}\left(\Delta^{n+j}, \partial \Delta^{n+j}\right)$ to $\mathcal{C}$ ell $(*)$. Now we define the map (3.1) by letting $\bar{s}$ go to the bordism class of $s_{0}$ ([LM13, Definitions 4.1 
and 4.2]. [LM13, Proposition 16.4(i), Remark 14.2(i) and page 44] show that this is a well-defined isomorphism which is compatible with the suspension maps as $n$ varies.

3.1. The ad theory of oriented topological manifolds. As motivation for the ad theory of IP-spaces, we briefly recall the ad theory adsTop ([LM13, Example 3.5 and Section 6]; also see [LM, Section 2]). The target category $\mathcal{A}_{\mathrm{STop}}$ has as objects the compact oriented topological manifolds with boundary which are subsets of some $\mathbb{R}^{n}$. The morphisms between objects of the same dimension are the orientation-preserving homeomorphisms, and the other morphisms are the inclusions with image in the boundary.

To describe the set $\operatorname{ad}_{\text {STop }}^{k}(K)$ we need to recall two definitions from LM13, Section 5]. A $\mathbb{Z}$-graded category $\mathcal{A}$ is called balanced if it comes with a natural involutive bijection for all objects $A, B$ of different dimensions

$$
\eta: \mathcal{A}(A, B) \rightarrow \mathcal{A}(A, i(B))
$$

which commutes with the involution $i$; examples are $\mathcal{C}$ ell $(K)$ and $\mathcal{A}_{\mathrm{STop}}$. Functors between balanced categories are called balanced if they commute with $\eta$.

Let $\mathcal{C}$ ell $l^{b}(K)$ be the category whose objects are the (unoriented) cells of $K$ and whose morphisms are the inclusions. Let $\mathcal{A}_{\text {STop }}^{b}$ be the category whose objects are compact orientable topological manifolds, whose morphisms between objects of the same dimension are homeomorphisms, and whose other morphisms are the inclusions with image in the boundary. A balanced functor

induces a functor

$$
F: \mathcal{C} e l l(K) \rightarrow \mathcal{A}_{\mathrm{STop}}
$$

$$
F^{b}: \mathcal{C} e l l^{b}(K) \longrightarrow \mathcal{A}_{\mathrm{STop}}^{b} .
$$

We define $\operatorname{ad}_{\text {STop }}^{k}(K) \subset \operatorname{pre}_{\text {STop }}^{k}(K)$ to be the set of functors $F$ with the following properties.

(a) $F$ is balanced.

(b) If $\left(\sigma^{\prime}, o^{\prime}\right)$ and $(\sigma, o)$ are oriented cells with $\operatorname{dim} \sigma^{\prime}=\operatorname{dim} \sigma-1$, and if the incidence number $\left[o, o^{\prime}\right]$ is equal to $(-1)^{k}$, then the map

$$
F\left(\sigma^{\prime}, o^{\prime}\right) \rightarrow \partial F(\sigma, o)
$$

is orientation preserving.

(c) For each $\sigma, \partial F^{b}(\sigma)$ is the colimit in Top of $\left.F^{b}\right|_{\mathcal{C} e l l^{b}(\partial \sigma)}$.

It is shown in [LM13, Appendix B] and [LM, Section 11] that the spectrum $\mathbf{Q}_{\mathrm{STop}}$ (resp., the symmetric spectrum $\mathbf{M}_{\mathrm{STop}}$ ) obtained from this ad theory is weakly equivalent to the usual Thom spectrum MSTop (resp., as a symmetric spectrum).

Remark 3.2. There are many variations of classical bordism spectra which can be represented by Quinn spectra as well. For example, one can consult [BL16] for the case of bordism with singularities of Baas-Sullivan type. 


\section{The ad THEORY of IP-SPACES}

Recall Proposition A.2.

Definition 4.1. Let $X$ and $X^{\prime}$ be PL $\partial$-pseudomanifolds of dimensions $n, n^{\prime}$. A strong embedding $f: X \rightarrow X^{\prime}$ is a PL embedding for which $X[n-i]=$ $f^{-1}\left(\left(X^{\prime}\right)\left[n^{\prime}-i\right]\right)$ for $0 \leq i \leq n$.

Let $\mathcal{A}_{\mathrm{IP}}$ be the $\mathbb{Z}$-graded category whose objects are compact oriented $\partial$ IP-spaces which are subsets of some $\mathbb{R}^{n}$, whose morphisms between objects of the same dimension are the orientation-preserving PL homeomorphisms and whose other morphisms are the strong embeddings with image in the boundary. The involution $i$ reverses the orientation. Then $\mathcal{A}_{\mathrm{IP}}$ is a balanced $\mathbb{Z}$-graded category. (The requirement that the morphisms between objects of different dimensions are strong embeddings will not actually be used until the proof of Lemma 6.5(ii)). Before defining $\operatorname{ad}_{\mathrm{IP}}^{k}(K)$, we need a fact about PL topology which will be proved at the end of this section.

Lemma 4.2. Let $\mathcal{P}$ be the category whose objects are compact polyhedra and whose morphisms are $P L$ embeddings. Let $K$ be a ball complex and $G: \mathcal{C}_{\text {ell }}(K) \rightarrow \mathcal{P}$ a covariant functor such that, for every $\sigma$, the map

$$
\operatorname{colim}_{\tau \in \partial \sigma} G(\tau) \rightarrow G(\sigma)
$$

is a monomorphism. Then for every subcomplex $L$ of $K$

(i) the space $\operatorname{colim}_{\sigma \in L} G(\sigma)$ has a PL structure for which the maps $G(\sigma) \rightarrow$ colim $_{\sigma \in L} G(\sigma)$ for $\sigma \in L$ are $P L$ embeddings, and

(ii) the map

is a PL embedding.

$$
\underset{\sigma \in L}{\operatorname{colim}} G(\sigma) \rightarrow \operatorname{colim}_{\sigma \in K} G(\sigma)
$$

(iii) Suppose that $v, v^{\prime}$ are cells of $K$, that $x$ is a point of $G(v)$, that $y$ is a point of $G\left(v^{\prime}\right)$ which is not in the image of $\operatorname{colim}_{\tau \in \partial v^{\prime}} G(\tau) \rightarrow G\left(v^{\prime}\right)$, and that $x$ and $y$ map to the same point of $\operatorname{colim}_{\sigma \in K} G(\sigma)$. Then $v^{\prime} \subset v$.

Now let $\mathcal{A}_{\mathrm{IP}}^{b}$ be the category whose objects are compact orientable IPspaces with boundary, whose morphisms between objects of the same dimension are PL homeomorphisms, and whose other morphisms are the strong embeddings with image in the boundary. A balanced functor

$$
F: \mathcal{C} \text { ell }(K) \longrightarrow \mathcal{A}_{\mathrm{IP}}
$$

induces a functor

$$
F^{b}: \mathcal{C} e l l^{b}(K) \longrightarrow \mathcal{A}_{\mathrm{IP}}^{b}
$$

Definition 4.3. Let $K$ be a ball complex. Define $\operatorname{ad}_{\mathrm{IP}}^{k}(K) \subset \operatorname{pre}_{\mathrm{IP}}^{k}(K)$ to be the set of functors $F$ with the following properties:

(a) $F$ is balanced.

(b) If $\left(\sigma^{\prime}, o^{\prime}\right)$ and $(\sigma, o)$ are oriented cells with $\operatorname{dim} \sigma^{\prime}=\operatorname{dim} \sigma-1$, and if the incidence number $\left[o, o^{\prime}\right]$ is equal to $(-1)^{k}$, then the map

$$
F\left(\sigma^{\prime}, o^{\prime}\right) \longrightarrow \partial F(\sigma, o)
$$


is orientation preserving.

(c) For each $\sigma \in K$, the map

$$
\operatorname{colim}_{\tau \in \partial \sigma} F^{b}(\tau) \rightarrow \partial F^{b}(\sigma)
$$

is a bijection.

Theorem 4.4. $\operatorname{ad}_{I P}$ is an ad theory.

Remark 4.5. By [F, Lemma 2.11.7], the Cartesian product of $\partial$-IP-spaces is a $\partial$-IP-space, and the product of an element of $\operatorname{ad}_{\mathrm{IP}}^{k}(K)$ with an element of $\operatorname{ad}_{\mathrm{IP}}^{l}(L)$ is an element of $\operatorname{ad}_{\mathrm{IP}}^{k+l}(K \times L)$. Thus ad IP is a multiplicative ad theory ([LM13, Definition 18.4]) and the associated symmetric spectrum $\mathbf{M}_{\mathrm{IP}}$ is a symmetric ring spectrum ([LM13, Theorem 18.5]).

Moreover, $\operatorname{ad}_{\mathrm{IP}}$ is a commutative ad theory ([LM, Definition 3.3]), so by Theorem 1.1 of $[\mathrm{LM}]$ there is a commutative symmetric ring spectrum $\mathbf{M}_{\mathrm{IP}}^{\text {comm }}$ which is weakly equivalent as a symmetric ring spectrum to $\mathbf{M}_{\mathrm{IP}}$. Specifically, there is a symmetric ring spectrum $\mathbf{A}$ and ring maps

$$
\mathbf{M}_{\mathrm{IP}} \leftarrow \mathbf{A} \rightarrow \mathbf{M}_{\mathrm{IP}}^{\text {comm }}
$$

which are weak equivalences.

Proof of Theorem 4.4. The only parts of [LM13, Definition 3.10] which are not obvious are (f) (the gluing axiom) and (g) (the cylinder axiom).

For part (g), let $F$ be a $K$-ad; we need to define $J(F): \mathcal{C}$ ell $(K \times I) \rightarrow \mathcal{A}_{\mathrm{IP}}$. First note that the statement of part $(\mathrm{g})$ specifies what $J(F)$ has to be on the subcategories $\mathcal{C}$ ell $(K \times 0)$ and $\mathcal{C}$ ell $(K \times 1)$. The remaining objects have the form $\left(\sigma \times I, o \times o^{\prime}\right)$ and we define $J(F)$ for such an object to be $F(\sigma, o) \times\left(I, o^{\prime}\right)$, where $\left(I, o^{\prime}\right)$ denotes the PL $\partial$-manifold $I$ with orientation $o^{\prime} . F(\sigma, o) \times I$ is a $\partial$-IP-space because the link at a point $(x, t)$ is the link in $F(\sigma, o)$ at $x$. The inclusions of $F(\sigma, o) \times\{0\}$ and $F(\sigma, o) \times\{1\}$ in $F(\sigma, o) \times I$ are strong embeddings (see Definition 4.1) by the definition of the stratification in Proposition A.2.

For part (f), let $K$ be a ball complex and $K^{\prime}$ a subdivision of $K$. Let $F$ be a $K^{\prime}$-ad. We need to show that there is a $K$-ad $E$ which agrees with $F$ on each residual subcomplex of $K$. We may assume (by induction over the lowest dimensional cell of $K$ that is not a cell of $K^{\prime}$ ) that $|K|$ is a PL $n$-ball, that $K$ has exactly one $n$-cell, and that $K^{\prime}$ is a subdivision of $K$ which agrees with $K$ on the boundary of $|K|$. Let $L$ be the subcomplex of $K^{\prime}$ consisting of cells in the boundary of $|K|$.

Now let $\tau$ denote the $n$-cell of $K$, and choose an orientation $o$ of $\tau$. To specify the $K$-ad $E$, we only need to define $E(\tau, o)$.

Let $X$ denote colim ${ }_{\sigma \in K^{\prime}} F^{b}(\sigma)$ and give $X$ the PL structure provided by Lemma 4.2(i). By Lemma 4.6 below, $X$ is a PL $\partial$-pseudomanifold.

We will write $\left[F^{b}(\sigma)\right]$ for the image of $F^{b}(\sigma)$ in $X$; by Lemma 4.2(i) this is a $\mathrm{PL} \partial$-pseudomanifold.

We claim that $X$ is a $\partial$-IP-space. By Definition 2.9, we need to show that $X-\partial X$ is an IP-space. We give $X-\partial X$ the intrinsic stratification (see 
Proposition A.1(iv)). Let $x \in X-\partial X$. By Lemma 4.2(iii) there is a unique $\sigma \in K^{\prime}-L$ for which $x$ is in the interior of $\left[F^{b}(\sigma)\right]$; give $\left[F^{b}(\sigma)\right]$ the intrinsic stratification and let $U$ be a distinguished neighborhood of $x$ in $\left[F^{b}(\sigma)\right]$. The proof of [LM13, Proposition 6.6] shows that $x$ has a neighborhood $V$ in $X$ such that there is a PL homeomorphism

$$
f: V \rightarrow U \times A,
$$

where $A$ is a Euclidean space. The filtration of $V$ inherited from $X$ is the same as the intrinsic stratification of $V$ by Proposition A.1(i), and (by Proposition A.1(ii) and (iii)) $f$ takes this filtration to the Cartesian product of the intrinsic stratification of $U$ with the trivial stratification of $A$. This implies that the link of $x$ in $X$ is the same as the link of $x$ in $\left[F^{b}(\sigma)\right]$, and so conditions (a) and (b) of Definition 2.7 are satisfied.

Now give the $n$-cells $\sigma$ of $K^{\prime}$ the orientations $o_{\sigma}$ which agree with $o$. Then $X$ has an orientation which agrees with the orientations of the $F\left(\sigma, o_{\sigma}\right)$, and we define $E(\tau, o)$ to be $X$ with this orientation. We claim that $E$ is a pre $K$-ad with values in $\mathcal{A}_{\mathrm{IP}}$. For this it only remains to show that $\left[F^{b}(\sigma)\right] \rightarrow X$ satisfies Definition 4.1 when $\sigma$ is a cell of $L$. We denote the stratification on a PL pseudomanifold (resp., PL $\partial$-pseudomanifold) $Y$ provided by Proposition A.1(iv) (resp., Proposition A.2) by $Y^{*}$ (resp., $Y[*]$ ). By its definition, the filtration $X[*]$ agrees (up to a dimension shift) with $(\partial X)^{*}$, so it suffices to show that $(\partial X)^{*}$ agrees with $\left[F^{b}(\sigma)\right][*]$. Next we observe that (by the proof of [LM13, Proposition 6.6]) each point of $\partial\left[F^{b}(\sigma)\right]$ has a neighborhood $U$ in $\partial\left[F^{b}(\sigma)\right]$ and a neighborhood $V$ in $\partial X$ with $V \approx U \times A$, where $A$ is a Euclidean space. Then $(\partial X)^{*}$ agrees with $V^{*}$ by Proposition A.1(i), and (by Proposition A.1(ii) and (iii)) the latter agrees with $U^{*} \times A$ (where $A$ is given the trivial filtration). This implies that $(\partial X)^{*}$ agrees with the restriction of $\left[F^{b}(\sigma)\right][*]$ to $\partial\left[F^{b}(\sigma)\right]$. Moreover, $(\partial X)^{*}$ also agrees with the restriction of $\left[F^{b}(\sigma)\right][*]$ to $\left[F^{b}(\sigma)\right]-\partial\left[F^{b}(\sigma)\right]$ by Proposition A.1(i), so the two filtrations agree on all of $\left[F^{b}(\sigma)\right]$.

Finally, we need to check that $E$ satisfies Definition 4.3. Parts (a) and (b) are obvious from the way $E$ was constructed, and part (c) is given by Lemma 4.6.

Lemma 4.6. With the PL structure given by Lemma 4.2(i),

(i) $X$ is a $P L$ d-pseudomanifold, and

(ii) the map

$$
\operatorname{colim}_{\sigma \in L} F^{b}(\sigma) \rightarrow \operatorname{colim}_{\sigma \in K^{\prime}} F^{b}(\sigma)=X
$$

is a $P L$ embedding with image $\partial X$.

Proof of Lemma 4.6. It follows from Lemma 4.2(i) that there is a triangulation of $X$ for which each $\left[F^{b}(\sigma)\right]$ is a subcomplex. The top-dimensional simplices have dimension $n-k$, where $k$ is the degree of $F$.

The proof of [Wh78, II.6.2] shows that $K^{\prime}$ has the following properties (where "cell" means closed cell): 
(1) Every cell is contained in an $n$-cell.

(2) Every $(n-1)$-cell in $L$ is contained in exactly one $n$-cell of $K^{\prime}$.

(3) Every $(n-1)$-cell not in $L$ is contained in exactly two $n$-cells.

To see that $X$ satisfies part (a) of Definition 2.2, let $s$ be any simplex of $X$. Then $s$ is contained in $\left[F^{b}(\sigma)\right]$ for some $\sigma$, and (1) implies that $\sigma \subset \sigma^{\prime}$ for some $n$-dimensional $\sigma^{\prime}$. Since $\left[F^{b}\left(\sigma^{\prime}\right)\right]$ is a PL $\partial$-pseudomanifold of dimension $n-k$, it has an $(n-k)$-dimensional simplex which contains $s$ as required.

For part (b), let $s$ be an $n-k-1$ simplex of $X$.

First suppose that $s$ is not contained in any $\left[F^{b}(\sigma)\right]$ with $\operatorname{dim}(\sigma)=n-1$. Then by (1) and Lemma 4.2(iii), there is a unique $\sigma$ of dimension $n$ for which $s$ is contained in $\left[F^{b}(\sigma)\right]$, and since $\left[F^{b}(\sigma)\right]$ is a PL $\partial$-pseudomanifold of dimension $n-k$ it has exactly two $(n-k)$-dimensional simplices which contain $s$.

Next suppose that $s \subset\left[F^{b}(\sigma)\right]$ with $\operatorname{dim}(\sigma)=n-1$. By Lemma 4.2(iii) there can only be one such $\sigma$. If $\sigma \in L$, then by (2) there is exactly one $n$ cell $\sigma^{\prime}$ containing $\sigma .\left[F^{b}\left(\sigma^{\prime}\right)\right]$ has exactly one $(n-k)$ simplex containing $s$, and Lemma 4.2 (iii) implies that this is the only $(n-k)$ simplex of $X$ which contains $s$. If $\sigma \notin L$, then by (3) there are exactly two $n$ cells $\sigma^{\prime}$ and $\sigma^{\prime \prime}$ which contain $s$. Each of $\left[F^{b}\left(\sigma^{\prime}\right]\right.$ and $\left[F^{b}\left(\sigma^{\prime \prime}\right)\right]$ has exactly one $(n-k)$ simplex which contains $s$, and Lemma 4.2(iii) implies that these are the only $(n-k)$ simplices of $X$ which contain $s$.

For part (c), we first observe that the proof of part (b) shows that

$$
\partial X=\bigcup_{\sigma \in L}\left[F^{b}(\sigma)\right]
$$

The rest of the proof for part (c) is similar to that for parts (a) and (b), but using [Wh78, II.6.2] instead of (1), (2) and (3).

For part (d), we first observe that the proof of [LM13, Proposition 6.6] shows that $\partial X$ is locally collared (in the sense of [RS72, page 24]); now [RS72, Theorem 2.25] shows that $\partial X$ is collared.

Finally part (ii) of the lemma follows from Lemma 4.2(ii) and Equation (4.1).

It remains to prove Lemma 4.2. The main ingredient is the following, which is Exercise 2.27(2) in RS72.

Lemma 4.7. Let $P, Q$ and $R$ be polyhedra and let $f: R \hookrightarrow P, g: R \hookrightarrow Q$ be $P L$ embeddings. Then the pushout of

$$
P \hookleftarrow R \hookrightarrow Q
$$

formed in the category of topological spaces, has a PL structure for which the inclusions of $P$ and $Q$ are $P L$ embeddings.

Proof of Lemma 4.2. First we prove (i) and (ii). Assume inductively that (i) and (ii) hold for any ball complex with at most $k$ cells. Let $K$ be a 
ball complex with $k+1$ cells and let $\sigma \in K$ be a top-dimensional cell. Let $K_{0}=K-\{\sigma\}$. Let $P=\operatorname{colim}_{\tau \in K_{0}} G(\tau)$ and $R=\operatorname{colim}_{\tau \subset \partial \sigma} G(\tau)$; the inductive hypothesis implies that $P$ and $R$ have PL structures for which all maps $G(\tau) \rightarrow P$ and $G(\tau) \rightarrow R$ are PL embeddings, and it also implies that $R \rightarrow P$ is a $\mathrm{PL}$ embedding. We are given that the map $R \rightarrow G(\sigma)$ is a monomorphism, and it is PL since its restriction to each $G(\tau)$ is PL. Now let $S$ denote $\operatorname{colim}_{\tau \in K} G(\tau)$. Then $S$ is the pushout of

$$
P \hookleftarrow R \hookrightarrow G(\sigma),
$$

so by Lemma 4.7 it has a PL structure for which $P \rightarrow S$ and $G(\sigma) \rightarrow S$ are PL maps; it follows that $G(\tau) \rightarrow S$ is a PL map for every $\tau$. It remains to check that part (ii) of Lemma 4.2 holds, so let $L$ be a subcomplex of $K$. The map

$$
i: \operatorname{colim}_{\tau \in L} G(\tau) \rightarrow S
$$

is $\mathrm{PL}$, since its restriction to each $G(\tau)$ is $\mathrm{PL}$, so we only need to check that $i$ is a monomorphism. If $\sigma \notin L$ this follows from the inductive hypothesis and the fact that $P \rightarrow S$ is a monomorphism. If $\sigma \in L$ then $\operatorname{colim}_{\tau \in L} G(\tau)$ is the pushout of

$$
\operatorname{colim}_{\tau \in L-\{\sigma\}} G(\tau) \hookleftarrow R \hookrightarrow G(\sigma),
$$

and this pushout maps by a monomorphism to the pushout of

$$
P \hookleftarrow R \hookrightarrow G(\sigma)
$$

which is $S$.

It remains to prove (iii). Suppose that $v^{\prime} \not \subset v$ and let $L$ be the minimal subcomplex of $K$ that contains $v$ and $v^{\prime}$. By (ii), $x$ and $y$ map to the same point of $\operatorname{colim}_{\sigma \in L} G(\sigma)$. But this implies that $x$ and $y$ are related by a sequence of elementary relations of the form $a \sim G\left(i_{\sigma, \sigma^{\prime}}\right)(b)$, where $\sigma \subset \sigma^{\prime} \in L$ and $i_{\sigma, \sigma^{\prime}}$ is the inclusion map. This is a contradiction, since $y$ cannot be part of such an elementary relation.

\section{5. ad THEORIES OF SyMmetric Poincaré COMPleXeS}

The ad theory constructed in the previous section gives a spectrum $\mathbf{Q}_{\mathrm{IP}}$ and a symmetric spectrum $\mathbf{M}_{\mathrm{IP}}$. Our next goal is to use the symmetric signature to construct maps (in the derived category of spectra and the derived category of symmetric spectra) from $\mathbf{Q}_{\mathrm{IP}}$ and $\mathbf{M}_{\mathrm{IP}}$ to suitable versions of the symmetric L-theory spectrum of $\mathbb{Z}$. In order to do this we need an ad theory for symmetric Poincaré complexes over $\mathbb{Z}$. In [LM13, Section 9] we gave an ad theory (denoted $\mathrm{ad}^{\mathbb{Z}}$ ) which was suggested by definitions from WW89. and [Ran92]; in particular this leads to a spectrum $\mathbf{Q}^{\mathbb{Z}}$ which is identical to Ranicki's spectrum $\mathbb{L}^{\bullet}(\mathbb{Z})$. But this turns out not to be well-adapted to questions of commutativity (see the beginning of [LM, Section 12]) or to intersection homology (see the introduction to [FM13b]), so in LM, Section $12]$ the second and third authors introduced a modification $\mathrm{ad}_{\mathrm{rel}}^{\mathbb{Z}}$ (rel stands for "relaxed") which gives a spectrum $\mathbf{Q}_{\text {rel }}^{\mathbb{Z}}$ weakly equivalent to Ranicki's 
$\mathbb{L}^{\bullet}(\mathbb{Z})$. In this section we review this material; we should mention that everything extends from $\mathbb{Z}$ to an arbitrary ring-with-involution $R$ and that [LM13] and [LM] develop the theory in this generality.

5.1. The ad theory $\operatorname{ad}^{\mathbb{Z}}$. As motivation we begin with the theory ad ${ }^{\mathbb{Z}}$. A chain complex over $\mathbb{Z}$ is called finite if it is free abelian and finitely generated in each degree and nonzero in only finitely many degrees; it is called homotopy finite if it is free abelian in each degree and chain homotopic to a finite complex. Let $\mathcal{D}$ be the category of homotopy finite chain complexes and chain maps. Let $W$ be the standard free resolution of $\mathbb{Z}$ by $\mathbb{Z}[\mathbb{Z} / 2]$ modules. The $n$-dimensional objects of the target category $\mathcal{A}^{\mathbb{Z}}$ are pairs $(C, \varphi)$, where $C$ is an object of $\mathcal{D}$ and

$$
\varphi: W \rightarrow C \otimes C
$$

is a $\mathbb{Z} / 2$-equivariant chain map which raises degrees by $n$. Here, the $\mathbb{Z} / 2$ action on $C \otimes C$ switches the factors. The morphisms $(C, \varphi) \rightarrow\left(C^{\prime}, \varphi^{\prime}\right)$ are the chain maps $f: C \rightarrow C^{\prime}$, with the additional requirement that $(f \otimes f) \circ \varphi=$ $\varphi^{\prime}$ when the dimensions are equal. The involution reverses the sign of $\varphi$. Next, $\left(\operatorname{ad}^{\mathbb{Z}}\right)^{k}(K) \subset\left(\text { pre }^{\mathbb{Z}}\right)^{k}(K)$ is defined to be the set of functors $F$ with the following properties:

(a) $F$ is balanced. This allows us to write $F(\sigma, o)$ as $\left(C_{\sigma}, \varphi_{\sigma, o}\right)$.

(b) $F$ is well-behaved, that is, each map $C_{\tau} \rightarrow C_{\sigma}$ is a split monomorphism in each dimension, and (writing $C_{\partial \sigma}$ for $\operatorname{colim}_{\tau \subset \partial \sigma} C_{\tau}$ ) each map

$$
C_{\partial \sigma} \rightarrow C_{\sigma}
$$

is a split monomorphism in each dimension.

(c) $F$ is closed, that is, for each cell $\sigma$ of $K$ the graded homomorphism from the cellular chain complex $\operatorname{cl}(\sigma)$ to $\operatorname{Hom}\left(W, C_{\sigma} \otimes C_{\sigma}\right)$ which takes $(\tau, o)$ to the composite

$$
W \stackrel{\varphi_{(\tau, o)}}{\longrightarrow} C_{\tau} \otimes C_{\tau} \rightarrow C_{\sigma} \otimes C_{\sigma}
$$

is a chain map. This implies that $\varphi_{\sigma, o}$ represents a class $\left[\varphi_{\sigma, o}\right]$ in $H_{n}\left(\operatorname{Hom}\left(W,\left(C_{\sigma} / C_{\partial \sigma}\right) \otimes C_{\sigma}\right)\right)$; the augmentation $\varepsilon: W \rightarrow \mathbb{Z}$ is a quasiisomorphism and hence induces an isomorphism

$$
\varepsilon^{*}: H_{n}\left(\left(C_{\sigma} / C_{\partial \sigma}\right) \otimes C_{\sigma}\right) \rightarrow H_{n}\left(\operatorname{Hom}\left(W,\left(C_{\sigma} / C_{\partial \sigma}\right) \otimes C_{\sigma}\right)\right) ;
$$

we denote $\left(\varepsilon^{*}\right)^{-1}\left[\varphi_{\sigma, o}\right]$ by $\left.\mathbf{c}\right]^{2}$

(d) $F$ is nondegenerate, that is, for each $\sigma$ the slant product with $\mathbf{c}$ gives an isomorphism

$$
H^{*}\left(\operatorname{Hom}\left(C_{\sigma}, \mathbb{Z}\right)\right) \rightarrow H_{\operatorname{dim} \sigma-k-*}\left(C_{\sigma} / C_{\partial \sigma}\right) .
$$

\footnotetext{
${ }^{2}$ Since different notation was used in LM13], we should explain how $\mathbf{c}$ is related to the element denoted by $\bar{\phi}_{*}(\mathbf{i})$ in [LM13, Section 9]. First note that, if $\iota$ is the map $\mathbb{Z} \rightarrow W$ which takes 1 to 1 , then $\mathbf{i}$ can be taken to be $\iota_{*}(1)$. Now the isomorphism $H_{n}\left(\left(C_{\sigma} / C_{\partial \sigma}\right) \otimes C_{\sigma}\right) \cong H_{n}\left(\operatorname{Hom}\left(\mathbb{Z},\left(C_{\sigma} / C_{\partial \sigma}\right) \otimes C_{\sigma}\right)\right) \stackrel{\varepsilon^{*}}{\longrightarrow} H_{n}\left(\operatorname{Hom}\left(W,\left(C_{\sigma} / C_{\partial \sigma}\right) \otimes C_{\sigma}\right)\right)$ takes $\bar{\phi}_{*}(\mathbf{i})$ to the class represented by $\bar{\phi} \circ \iota \circ \varepsilon$, which is the same as the class represented by $\bar{\phi}$ (since $\iota \circ \varepsilon$ is chain homotopic to the identity $W \rightarrow W$ ), and this is the same as the class we have denoted by $\left[\varphi_{\sigma, o}\right]$. Thus $\bar{\phi}_{*}(\mathbf{i})=\left(\varepsilon^{*}\right)^{-1}\left[\varphi_{\sigma, o}\right]=\mathbf{c}$.
} 
Remark 5.1. In [LM13, the second and third authors give a construction of a symmetric signature map $\mathbf{Q}_{\mathrm{STop}} \rightarrow \mathbf{Q}^{\mathbb{Z}}$ in the derived category of spectra, using ideas from Ran92 (see [LM13, Section 10 and the end of Section 8]). The starting point for this construction is the observation that, if $M$ is a compact oriented $\partial$-manifold and $\xi \in S_{n}(M)$, where $S_{*}(-)$ denotes singular chains, represents the fundamental class of $M$ then the composite

$$
W \cong W \otimes \mathbb{Z} \stackrel{1 \otimes \xi}{\longrightarrow} W \otimes S_{*} M \stackrel{E A W}{\longrightarrow} S_{*} M \otimes S_{*} M
$$

(where $E A W$ is the extended Alexander-Whitney map, which can be constructed using acyclic models) is an object of $\mathcal{A}^{\mathbb{Z}}$. The Alexander-Whitney map (and a fortiori the extended Alexander-Whitney map) does not exist for intersection chains, which is one reason we need the modification of $\mathcal{A}^{\mathbb{Z}}$ given in the next subsection.

Remark 5.2. The ad theory $\mathrm{ad}^{\mathbb{Z}}$ is multiplicative ([LM13, Definitions 18.1 and 9.12]) but not commutative (see the beginning of Section 12 of [LM]).

5.2. The ad theory $\mathrm{ad}_{\mathrm{rel}}^{\mathbb{Z}}$. An object of the category $\mathcal{A}_{\text {rel }}^{\mathbb{Z}}$ is a quadruple $(C, D, \beta, \varphi)$, where $C$ is an object of $\mathcal{D}, D$ is a chain complex with a $\mathbb{Z} / 2$-action, $\beta$ is a quasi-isomorphism $C \otimes C \rightarrow D$ which is also a $\mathbb{Z} / 2$ equivariant map, and $\varphi$ is an element of the fixed point set $D_{n}^{\mathbb{Z} / 2}$. A morphism $(C, D, \beta, \varphi) \rightarrow\left(C^{\prime}, D^{\prime}, \beta^{\prime}, \varphi^{\prime}\right)$ is a pair $\left(f: C \rightarrow C^{\prime}, g: D \rightarrow D^{\prime}\right)$, where $f$ and $g$ are chain maps, $g$ is $\mathbb{Z} / 2$-equivariant, $g \beta=\beta^{\prime}(f \otimes f)$, and (if the dimensions are equal) $g(\varphi)=\varphi^{\prime}$.

Example 5.3. If $(C, \varphi)$ is an object of $\mathcal{A}^{\mathbb{Z}}$ then the quadruple $(C,(C \otimes$ $\left.C)^{W}, \beta, \varphi\right)$ is a relaxed quasi-symmetric complex, where

$$
(C \otimes C)^{W}=\operatorname{Hom}(W, C \otimes C)
$$

is the $\mathbb{Z} / 2$-chain complex with fixed points the equivariant chain maps and $\beta: C \otimes C \rightarrow(C \otimes C)^{W}$ is induced by the augmentation $W \rightarrow \mathbb{Z}$. This construction gives a functor $\mathcal{A}^{\mathbb{Z}} \rightarrow \mathcal{A}_{\text {rel }}^{\mathbb{Z}}$.

Example 5.4. In the situation of Remark 5.1, we obtain an object of $\mathcal{A}_{\text {rel }}^{\mathbb{Z}}$ by letting $C$ be $S_{*} M, D$ be $S_{*}(M \times M), \beta$ be the cross product, and $\varphi$ be the image of $\xi \in S_{n}(M)$ under the diagonal map.

Now $\left(\operatorname{ad}_{\mathrm{rel}}^{\mathbb{Z}}\right)^{k}(K) \subset\left(\operatorname{pre}_{\mathrm{rel}}^{\mathbb{Z}}\right)^{k}(K)$ is defined to be the set of functors $F$ with the following properties:

(a) $F$ is balanced. This allows us to write $F(\sigma, o)$ as $\left(C_{\sigma}, D_{\sigma}, \beta_{\sigma}, \varphi_{\sigma, o}\right)$.

(b) $F$ is well-behaved, that is, all maps $C_{\tau} \rightarrow C_{\sigma}, D_{\tau} \rightarrow D_{\sigma}, C_{\partial \sigma} \rightarrow C_{\sigma}$ and $D_{\partial \sigma} \rightarrow D_{\sigma}$ are split monomorphisms in each dimension. This implies that the map $\beta_{*}: H_{*}\left(C_{\sigma} \otimes C_{\sigma},(C \otimes C)_{\partial \sigma}\right) \rightarrow H_{*}\left(D_{\sigma}, D_{\partial \sigma}\right)$ is an isomorphism ([LM, Lemma 12.7(ii)]).

(c) $F$ is closed, that is, for each $\sigma$ the map

$$
\operatorname{cl}(\sigma) \rightarrow D_{\sigma}
$$


which takes $\langle\tau, o\rangle$ to $\varphi_{\tau, o}$ is a chain map. This implies that $\varphi_{\sigma, o}$ represents a class $\left[\varphi_{\sigma, o}\right]$ in $H_{n}\left(D_{\sigma}, D_{\partial \sigma}\right)$.

(d) $F$ is nondegenerate, that is, for each $\sigma$ the slant product with $\left(\beta_{*}\right)^{-1}\left(\left[\varphi_{\sigma, o}\right]\right)$ is an isomorphism

$$
H^{*}\left(\operatorname{Hom}\left(C_{\sigma}, \mathbb{Z}\right)\right) \rightarrow H_{\operatorname{dim} \sigma-k-*}\left(C_{\sigma} / C_{\partial \sigma}\right) .
$$

Remark 5.5. (i) The functor $\mathcal{A}^{\mathbb{Z}} \rightarrow \mathcal{A}_{\text {rel }}^{\mathbb{Z}}$ in Example 5.3 gives a map of spectra $\mathbf{Q}^{\mathbb{Z}} \rightarrow \mathbf{Q}_{\text {rel }}^{\mathbb{Z}}$ which is a weak equivalence ([LM, Section 13]).

(ii) The ad theory ad $\mathrm{rel}_{\mathrm{rel}}^{\mathbb{Z}}$ is commutative ([LM, Definition 3.3 and Remark 12.13]) so Theorem 1.1 of [LM] shows that there is a commutative symmetric ring spectrum $\left(\mathbf{M}_{\mathrm{rel}}^{\mathbb{Z}}\right)^{\text {comm }}$ which is weakly equivalent as a symmetric ring spectrum to $\mathbf{M}_{\text {rel }}^{\mathbb{Z}}$.

5.3. Connective versions. In the sequel, we will mostly deal with connective versions of $L$-theory rather than with periodic ones. There is a general procedure which takes an ad theory to another ad theory and which makes the associated Quinn spectrum connective: define the sub functor $\mathrm{ad}_{\geq 0}$ of an ad theory by

$$
\operatorname{ad}_{\geq 0}^{k}(K, L)=\operatorname{ad}^{k}\left(K, L \cup K^{(k-1)}\right),
$$

where $K^{(n)}$ denotes the $n$-skeleton of $K$. We leave it to the reader to check the properties of an ad theory for $\operatorname{ad}_{\geq 0}$.

In order to see that this has the desired effect on homotopy groups, we recall from [LM13, Theorem 16.1] that the homotopy group $\pi_{k} \mathbf{Q}$ of a Quinn spectrum coincides with the $k$-th bordism group. The bordism group is obtained from the set of $(-k)$-dimensional $*$-ads by identifying two elements if there is an $I$-ad which restricts to the given ones at the ends. For $K=*$ or $I$ we have $\operatorname{ad}_{\geq 0}^{-k}(K)=\operatorname{ad}^{-k}(K)$ for $k \geq 0$ and $\operatorname{ad}_{\geq 0}^{-k}(*)=\left\{\emptyset_{-k}\right\}$ for $k<0$ and the claim follows.

If the nonempty objects of the category $\mathcal{A}$ are concentrated in nonnegative dimensions then all ad theories with values in $\mathcal{A}$ are connective; this is the case for $\mathcal{A}_{S T \text { To }}$ and $\mathcal{A}_{I P}$. For the theory ad ${ }^{\mathbb{Z}}$, there is a map from the Quinn spectrum $\mathbf{Q}_{>0}^{\mathbb{Z}}$ to the connective spectrum $\mathbb{L}=\mathbf{L} \bullet\langle 0\rangle(\mathbb{Z})$ which is a weak equivalence ( the two spectra are not identical because in Ran92, Definition 15.2] the restriction to the $(k-1)$ skeleton is required to be acyclic but not zero).

A morphism of ad theories induces a morphism of their connective versions. Any morphism from a connective ad theory to another ad theory takes values in the connective version.

If the theory ad is multiplicative (resp. commutative) then so is $\mathrm{ad}_{>0}$. There is a map of Quinn spectra $\mathbf{Q}_{>0} \rightarrow \mathbf{Q}$ and a map of symmetric spectra $\mathbf{M}_{\geq 0} \rightarrow \mathbf{M}$ which is a ring map if ad is multiplicative and weakly equivalent to an $E_{\infty}$ map if ad is commutative. 


\section{The Symmetric Signature AS A MAP OF SPECTRA}

In this section we construct symmetric signature maps

$$
\text { Sig : } \mathbf{Q}_{\mathrm{IP}} \rightarrow \mathbf{Q}_{\mathrm{rel}, \geq 0}^{\mathbb{Z}}
$$

(in the derived category of spectra) and

$$
\text { Sig }: \mathbf{M}_{\mathrm{IP}} \rightarrow \mathbf{M}_{\mathrm{rel}, \geq 0}^{\mathbb{Z}}
$$

(in the derived category of symmetric spectra). The first step is to give a variant of the ad theory $\operatorname{ad}_{\mathrm{IP}}$. As we have seen in Remark 5.1] and Example 5.4, in order for a compact oriented $\partial$-manifold to give rise to an object of $\mathcal{A}_{\text {rel }}^{\mathbb{Z}}$ we must choose a chain representative for the fundamental class. The same is true for $\partial$-IP-spaces, so in Subsection 6.1 we construct a suitable ad theory $\operatorname{ad}_{\text {IPFun }}$ and we show that the forgetful maps $\mathbf{Q}_{\text {IPFun }} \rightarrow \mathbf{Q}_{\text {IP }}$ and $\mathbf{M}_{\mathrm{IPFun}} \rightarrow \mathbf{M}_{\mathrm{IP}}$ are weak equivalences. Next, in Subsection 6.3 we construct a functor

$$
\operatorname{sig}: \mathcal{A}_{\text {IPFun }} \rightarrow \mathcal{A}_{\text {rel }}^{\mathbb{Z}}
$$

which induces a natural transformation

$$
\text { sig : } \operatorname{ad}_{\text {IPFun }}(K) \rightarrow \operatorname{ad}_{\mathrm{rel}, \geq 0}^{\mathbb{Z}}(K)
$$

for strict ball complexes $K$ (see [LM, third paragraph of Section 14 and Remark 14.1] for the meaning and significance of strictness). These results allow us to make the following definition.

Definition 6.1. The symmetric signature map

$$
\text { Sig : } \mathbf{Q}_{\mathrm{IP}} \rightarrow \mathbf{Q}_{\mathrm{rel}, \geq 0}^{\mathbb{Z}}
$$

is the composite

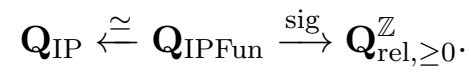

The symmetric signature map

$$
\text { Sig }: \mathbf{M}_{\mathrm{IP}} \rightarrow \mathbf{M}_{\mathrm{rel}, \geq 0}^{\mathbb{Z}}
$$

is the composite

$$
\mathbf{M}_{\mathrm{IP}} \stackrel{\simeq}{\stackrel{\sim}{\longleftarrow}} \mathbf{M}_{\mathrm{IPFun}} \stackrel{\text { sig }}{\longrightarrow} \mathbf{M}_{\mathrm{rel}, \geq 0}^{\mathbb{Z}} .
$$

6.1. The ad theory ad $\mathrm{IPFun}_{\mathrm{P}}$. We denote singular intersection chains with perversity $\bar{p}$ by $I S_{*}^{\bar{p}}$. By [FM13a, Proposition 7.7], an orientation of a compact $n$-dimensional $\partial$-IP-space $X$ determines a fundamental class $\Gamma_{X} \in I H_{n}^{\overline{0}}(X, \partial X ; \mathbb{Z})$, where $\overline{0}$ denotes the 0 perversity.

We define a category $\mathcal{A}_{\text {IPFun }}$ as follows. The objects are pairs $(X, \xi)$, where $X$ is a compact oriented $\partial$-IP-space and $\xi \in I S_{n}^{\overline{0}}(X ; \mathbb{Z})$ is a chain whose image in $I S_{n}^{\overline{0}}(X, \partial X ; \mathbb{Z})$ it represents the fundamental class $\Gamma_{X}$; there is also an empty object of dimension $n$ for each $n$. The morphisms $(X, \xi) \rightarrow\left(X^{\prime}, \xi^{\prime}\right)$ between objects of the same dimension are PL homeomorphisms which take $\xi$ to $\xi^{\prime}$, and the other morphisms are strong embeddings with image in the boundary. There is a forgetful functor $\mathcal{A}_{\mathrm{IPFun}} \rightarrow \mathcal{A}_{\mathrm{IP}}$, and we define $\operatorname{ad}_{\mathrm{IPFun}}^{k}(K) \subset \operatorname{pre}_{\mathrm{IPFun}}^{k}(K)$ to be the set of functors $F$ such that 
(a) the composite of $F$ with the forgetful functor is an element of $\operatorname{ad}_{\mathrm{IP}}^{k}(K)$, and

(b) for each oriented cell $(\sigma, o)$ of $K$, the equation

$$
\partial \xi_{\sigma, o}=\sum \xi_{\sigma^{\prime}, o^{\prime}}
$$

holds, where $\sigma^{\prime}$ runs through the cells of $\partial \sigma$ and $o^{\prime}$ is the orientation for which the incidence number $\left[o, o^{\prime}\right]$ is $(-1)^{k}$.

Proposition 6.2. $\operatorname{ad}_{\mathrm{IPFun}}$ is a connective ad theory.

Proof. We only need to check parts (f) and (g) of [LM13, Definition 3.10]. For the proof of (f) we use the gluing construction in the proof of Theorem 4.4 (and the notation there) and we define $\xi_{\tau, o}$ to be $\sum \xi_{\sigma, o_{\sigma}}$, where $\sigma$ runs throught the $n$-cells of $K^{\prime}$; then $\xi$ maps to a representative of $\Gamma_{X}$ by [FM13a, Corollary 5.16].

For $(\mathrm{g})$, let $F$ be a $K$-ad, and write $F(\sigma, o)=\left(X_{\sigma}, \xi_{\sigma, o}\right)$. As in the proof of Theorem 4.4, we only need to specify $J(F)$ on oriented cells of the form $\left(\sigma \times I, o \times o^{\prime}\right)$, and moreover we can assume that $o^{\prime}$ is the standard orientation. Let $s: \Delta^{1} \rightarrow I$ be the standard oriented homeomorphism, and define $J(F)\left(\sigma \times I, o \times o^{\prime}\right)=\left(X_{\sigma} \times I, \xi \times s\right)$.

The forgetful functor $\mathcal{A}_{\text {IPFun }} \rightarrow \mathcal{A}_{\text {IP }}$ gives rise to a morphism ad IPFun $\rightarrow$ $\operatorname{ad}_{I P}$ of ad theories.

Proposition 6.3. The maps

$$
\text { Q }
$$

and

$$
\mathrm{M}_{\mathrm{IPFun}} \rightarrow \mathrm{M}_{\mathrm{IP}}
$$

induced by the forgetful functor $\mathcal{A}_{\mathrm{IPFun}} \rightarrow \mathcal{A}_{\mathrm{IP}}$ are weak equivalences.

Proof. Recall the definition of the bordism groups of an ad theory ([LM13, Definitions 4.1 and 4.2]). By Remark 3.1 and [LM13, Proposition 17.7], it suffices to show that the map of bordism groups

$$
\left(\Omega_{\mathrm{IPFun}}\right)_{*} \rightarrow\left(\Omega_{\mathrm{IP}}\right)_{*}
$$

is an isomorphism. This map is obviously onto, and it is a monomorphism by the proof of [LM13, Lemma 8.2].

Remark 6.4. $\operatorname{ad}_{\text {IPFun }}$ is a commutative ad theory, so by Theorem 1.1 of [LM] there is a commutative symmetric ring spectrum $\mathbf{M}_{\mathrm{IPFun}}^{\mathrm{comm}}$ which is weakly equivalent as a symmetric ring spectrum to $\mathbf{M}_{\mathrm{IPFun}}$. Moreover, the forgetful map $\operatorname{ad}_{\text {IPFun }} \rightarrow \operatorname{ad}_{\text {IP }}$ is strictly multiplicative, so the proof of [LM, Theorem 1.1] gives a commutative diagram

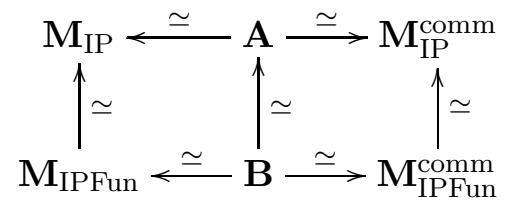


in which $\mathbf{A}$ and $\mathbf{B}$ are symmetric ring spectra and all arrows are ring maps.

6.2. Background. Before proceeding we need to recall some information about generalized perversities. For a stratified $n$-dimensional $\partial$ pseudomanifold $Y$ the components of $Y^{i}-Y^{i-1}$ are called $i$-dimensional strata; the $n$-dimensional strata are called regular and the others singular. Recall ([F, Definition 3.1.1]) that a generalized perversity ${ }^{3}$ on $Y$ is a function $\bar{p}$ from the set of strata of $Y$ to $\mathbb{Z}$ which is 0 on the regular strata; an ordinary perversity $\bar{q}$ can be thought of as a generalized perversity taking a stratum $S$ to $\bar{q}(\operatorname{codim}(S))$. We use the definition of intersection homology for general perversities given in [F, Definition 6.2.2]. Let $\bar{n}$ be the upper middle perversity.

Let $X$ be a $\partial$-IP-space, and give $X$ the stratification of Proposition A.2, Give $X \times X$ the product stratification. Define a generalized perversity $Q_{\bar{n}, \bar{n}}$ on $X \times X$ as follows.

$$
Q_{\bar{n}, \bar{n}}\left(S_{1} \times S_{2}\right)= \begin{cases}\bar{n}\left(S_{1}\right)+\bar{n}\left(S_{2}\right)+2, & S_{1}, S_{2} \text { both singular strata, } \\ \bar{n}\left(S_{1}\right), & S_{2} \text { a regular stratum and } S_{1} \text { singular, } \\ \bar{n}\left(S_{2}\right), & S_{1} \text { a regular stratum and } S_{2} \text { singular, } \\ 0, & S_{1}, S_{2} \text { both regular strata. }\end{cases}
$$

By [FM13a, Subsection 4.1], the diagonal map induces a chain map

$$
d: I S_{*}^{\overline{0}}(X ; \mathbb{Z}) \rightarrow I S_{*}^{Q \bar{n}, \bar{n}}(X \times X ; \mathbb{Z})
$$

(this is the reason we need generalized perversities). By $[\mathrm{F}$, Theorem 6.4.6 and Remark 6.4.7], the cross product induces an equivalence

$$
I S_{*}^{\bar{n}}(X ; \mathbb{Z}) \otimes I S_{*}^{\bar{n}}(X ; \mathbb{Z}) \rightarrow I S_{*}^{Q_{\bar{n}}, \bar{n}}(X \times X ; \mathbb{Z}) .
$$

\subsection{The functor sig : $\mathcal{A}_{\text {IPFun }} \rightarrow \mathcal{A}_{\text {rel }}^{\mathbb{Z}}$.}

Lemma 6.5. (i) Let $(X, \xi)$ be an object of $\mathcal{A}_{\text {IPFun. }}$. Give $X$ the stratification of Proposition $A .2$ and give $X \times X$ the product stratification. Then $(C, D, \beta, \varphi)$ is an object of $\mathcal{A}_{\mathrm{rel}}^{\mathbb{Z}}$, where

$$
\begin{aligned}
& C=I S_{*}^{\bar{n}}(X ; \mathbb{Z}), \\
& D=I S_{*}^{Q \bar{n}, \bar{n}}(X \times X ; \mathbb{Z}), \\
& \beta \text { is the cross product, and } \\
& \varphi \text { is the image of } \xi \text { under the diagonal map (6.1). }
\end{aligned}
$$

(ii) Let $f:(X, \xi) \rightarrow\left(X^{\prime}, \xi^{\prime}\right)$ be a morphism in $\mathcal{A}_{\mathrm{IPFun}}$ and let $(C, D, \beta, \varphi)$ and $\left(C^{\prime}, D^{\prime}, \beta^{\prime}, \varphi^{\prime}\right)$ be the objects of $\mathcal{A}_{\mathrm{rel}}^{\mathbb{Z}}$ corresponding to $(X, \xi)$ and $\left(X^{\prime}, \xi^{\prime}\right)$. Then $f$ induces a morphism $(C, D, \beta, \varphi) \rightarrow\left(C^{\prime}, D^{\prime}, \beta^{\prime}, \varphi^{\prime}\right)$.

Proof. (i) We only need to show that $I S_{*}^{\bar{n}}(X ; \mathbb{Z})$ is homotopy finite. The complex $I S_{*}^{\bar{n}}(X ; \mathbb{Z})$ is free because it is a subcomplex of the singular chain complex $S_{*}(X ; \mathbb{Z})$. Next let $T$ be a triangulation of $X$ which is compatible

\footnotetext{
${ }^{3}$ These are simply called perversities in $[\mathrm{F}]$.
} 
with the stratification of $X$. Let $I C_{*}^{T, \bar{n}}(X ; \mathbb{Z})$ denote the complex of PL intersection chains which are simplicial with respect to $T$. This is free, finitely generated in each degree, and nonzero in only finitely many degrees. By [F, Corollary 5.4.6], the inclusion

$$
I C_{*}^{T, \bar{n}}(X ; \mathbb{Z}) \rightarrow I S_{*}^{\bar{n}}(X ; \mathbb{Z})
$$

is a quasi-isomorphism. Since the domain and range are free, it is a chain homotopy equivalence, and thus $I S_{*}^{\bar{n}}(X ; \mathbb{Z})$ is homotopy finite.

(ii) If the dimensions are not equal then the definition of $\mathcal{A}_{\text {IPFun }}$ shows that $f$, and hence also $f \times f$, is a strong embedding, so they induce maps of intersection chains and the result follows. If the dimensions are equal then $f$ is a PL homeomorphism so, by Propositions A.1(iii) and A.2, $f$ and $f \times f$ preserve the filtrations and therefore induce maps of intersection chains.

Lemma 6.5 gives a functor

$$
\operatorname{sig}: \mathcal{A}_{\text {IPFun }} \rightarrow \mathcal{A}_{\text {rel }}^{\mathbb{Z}} \text {. }
$$

Proposition 6.6. If $K$ is strict and $F \in \operatorname{ad}_{\mathrm{IPFun}}^{k}(K)$ then $\operatorname{sig} \circ F \in$ $\left(\operatorname{ad}_{\mathrm{rel}, \geq 0}^{\mathbb{Z}}\right)^{k}(K)$.

The proposition gives the maps

$$
\operatorname{sig}: \mathbf{Q}_{\mathrm{IPFun}} \rightarrow \mathbf{Q}_{\mathrm{rel}, \geq 0}^{\mathbb{Z}}
$$

and

$$
\operatorname{sig}: \mathbf{M}_{\text {IPFun }} \rightarrow \mathbf{M}_{\mathrm{rel}, \geq 0}^{\mathbb{Z}}
$$

which are needed for Definition 6.1.

Proof of Proposition 6.6. Since sig $\circ F$ is closed by property (b) of the definition in Subsection 6.1, we only need to check that $\operatorname{sig} \circ F$ is well-behaved and nondegenerate. Write $F(\sigma, o)=\left(X_{\sigma, o}, \xi_{\sigma, o}\right)$. Let $Y_{\sigma}$ be the underlying $\partial$-IP-space of $X_{\sigma, o}$ (forgetting the orientation).

To show that sig $\circ F$ is well-behaved, we need to show that the functors $I S_{i}^{\bar{n}}\left(X_{\sigma} ; \mathbb{Z}\right)$ and $I S_{*}^{Q_{\bar{n}}, \bar{n}}\left(X_{\sigma} \times X_{\sigma} ; \mathbb{Z}\right)$ are well-behaved. We give the proof for the first of these functors; the proof for the second is similar.

First we need to know that for cells $\sigma, \tau$ of $K$ with $\sigma \subset \tau$ the monomorphism

$$
I S_{i}^{\bar{n}}\left(Y_{\sigma} ; \mathbb{Z}\right) \rightarrow I S_{i}^{\bar{n}}\left(Y_{\tau} ; \mathbb{Z}\right)
$$

is split for each $i$. For this it suffices to show that the quotient $I S_{i}^{\bar{n}}\left(Y_{\tau} ; \mathbb{Z}\right) / I S_{i}^{\bar{n}}\left(Y_{\sigma} ; \mathbb{Z}\right)$ is free, and this in turn follows from the fact that the map from this quotient to the free abelian group $S_{i}\left(Y_{\tau} ; \mathbb{Z}\right) / S_{i}\left(Y_{\sigma} ; \mathbb{Z}\right)$ is a monomorphism.

Next we need to know that for each cell $\sigma$ the map

$$
\underset{\sigma \subset \partial \tau}{\operatorname{colim}} I S_{i}^{\bar{n}}\left(Y_{\sigma} ; \mathbb{Z}\right) \rightarrow I S_{i}^{\bar{n}}\left(Y_{\tau} ; \mathbb{Z}\right)
$$


is a split monomorphism. It is a monomorphism by Lemma 6.7(iii). To see that it is split it suffices to show that the quotient

$$
I S_{i}^{\bar{n}}\left(Y_{\tau} ; \mathbb{Z}\right) / \operatorname{colim}_{\sigma \subset \partial \tau} I S_{i}^{\bar{n}}\left(Y_{\sigma} ; \mathbb{Z}\right)
$$

is free. By Lemma 6.7(iii) this is the same as the quotient

$$
I S_{i}^{\bar{n}}\left(Y_{\tau} ; \mathbb{Z}\right) / \sum_{\sigma \subset \partial \tau} I S_{i}^{\bar{n}}\left(Y_{\sigma} ; \mathbb{Z}\right)
$$

and by Lemma 6.7(ii) the map from this quotient to the free abelian group $S_{i}\left(Y_{\tau} ; \mathbb{Z}\right) / \sum_{\sigma \subset \partial \tau} S_{i}\left(Y_{\sigma} ; \mathbb{Z}\right)$ is a monomorphism. This concludes the proof that $I S_{i}^{\bar{n}}\left(Y_{\sigma} ; \mathbb{Z}\right)$ is well-behaved.

For nondegeneracy, we first observe that, by Lemma 6.7 below, the map

$$
\underset{\tau \in \partial \sigma}{\operatorname{colim}} I S_{*}^{\bar{n}}\left(Y_{\tau} ; \mathbb{Z}\right) \rightarrow I S_{*}^{\bar{n}}\left(\partial Y_{\sigma} ; \mathbb{Z}\right)
$$

is a quasi-isomorphism for every simplex $\sigma$ of $K$.

Now it suffices to show that the horizontal map in the following diagram is an isomorphism for each oriented simplex $(\sigma, o)$.

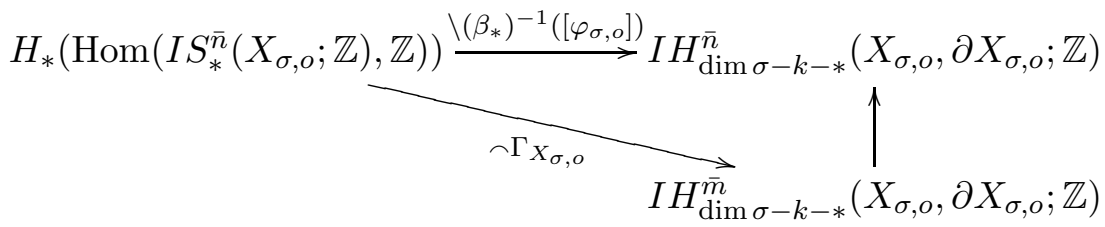

The construction of the cap product is given in Appendix D, and the fundamental class $\Gamma_{X_{\sigma, o}}$ is given by [FM13a, Proposition 7.7]. Inspection of the definitions shows that the diagram commutes, and the slanted arrow is an isomorphism by TheoremE.5, so we only need to show that the vertical arrow is an isomorphism. For this it suffices to show that the maps

$$
I H_{*}^{\bar{m}}\left(\partial X_{\sigma, o} ; \mathbb{Z}\right) \rightarrow I H_{*}^{\bar{n}}\left(\partial X_{\sigma, o} ; \mathbb{Z}\right)
$$

and

$$
I H_{*}^{\bar{m}}\left(X_{\sigma, o} ; \mathbb{Z}\right) \rightarrow I H_{*}^{\bar{n}}\left(X_{\sigma, o} ; \mathbb{Z}\right)
$$

are isomorphisms. The first of these is an isomorphism by Proposition 2.10 and the argument in GM83, Subsection 5.6.1]. To see that the second map is an isomorphism we oberve that if $W$ denotes $X_{\sigma, o}$ with a collar of the boundary removed then the maps $W \rightarrow X_{\sigma, o}$ and $W \rightarrow X_{\sigma, o}-\partial X_{\sigma, o}$ are stratified homotopy equivalences and therefore induce isomorphisms of intersection homology (see [FM13a, Appendix A], so it suffices to observe that the map

$$
I H_{*}^{\bar{m}}\left(X_{\sigma, o}-\partial X_{\sigma, o} ; \mathbb{Z}\right) \rightarrow I H_{*}^{\bar{n}}\left(X_{\sigma, o}-\partial X_{\sigma, o} ; \mathbb{Z}\right)
$$

is an isomorphism by the argument in GM83, Subsection 5.6.1]. 
Before stating the lemma let us recall that intersection homology and the notion of stratified homotopy can be defined for any filtered space, and that a stratified homotopy equivalence induces an isomorphism of intersection homology ([F03, Section 2]). Also recall that if $X$ is a functor from $\operatorname{Cell}(K)$ to PL spaces and $L$ is a subcomplex of $K$ then we write $X_{L}$ for $\operatorname{colim}_{\sigma \in L} X_{\sigma}$.

Note: part (i) of the following lemma is stated in more generality than is needed in this section (the case $U=X_{L^{\prime}}$ would suffice for that); the extra generality is needed for Subsection 10.9 .

Lemma 6.7. Let $F \in \operatorname{ad}_{\mathrm{IPFun}}^{k}(K)$, and write $F(\sigma, o)=\left(X_{\sigma}, \xi_{\sigma, o}\right)$. For every subcomplex $L$ of $K$, give $X_{L}$ the filtration which restricts to the filtration of Proposition A.2 on each $X_{\sigma}$. Then

(i) for every pair of subcomplexes $L^{\prime} \subset L$, and for every open set $U$ of $L^{\prime}$, there is a neighborhood $V$ of $U$ in $X_{L}$ such that $V \cap X_{L^{\prime}}=U$ and the inclusion $U \rightarrow V$ is a stratified deformation retract where the retraction $r: V \rightarrow U$ has the property that $r(x) \in X_{\sigma}$ whenever $x \in X_{\sigma}$, and

(ii) for every subcomplex $L$ and every $i \in \mathbb{Z}$, the intersection of $I S_{i}^{\bar{n}}\left(X_{K} ; \mathbb{Z}\right)$ and $\sum_{\sigma \in L} S_{i}\left(X_{\sigma} ; \mathbb{Z}\right)$ (considered as subgroups of $S_{i}\left(X_{K} ; \mathbb{Z}\right)$ ) is $\sum_{\sigma \in L} I S_{i}^{\bar{n}}\left(X_{\sigma}\right)$, and

(iii) if $K$ is strict then for every subcomplex $L$ the map

$$
\underset{\sigma \in L}{\operatorname{colim}} I S_{*}^{\bar{n}}\left(X_{\sigma} ; \mathbb{Z}\right) \rightarrow \sum_{\sigma \in L} I S_{*}^{\bar{n}}\left(X_{\sigma} ; \mathbb{Z}\right)
$$

is an isomorphism, and

(iv) for every subcomplex $L$ the map

$$
\underset{\sigma \in L}{\operatorname{colim}} I S_{*}^{\bar{n}}\left(X_{\sigma} ; \mathbb{Z}\right) \rightarrow I S_{*}^{\bar{n}}\left(X_{L}\right)
$$

is a quasi-isomorphism.

Remark 6.8. For the proof of part (iv) we will use the following fact: given a commutative diagram

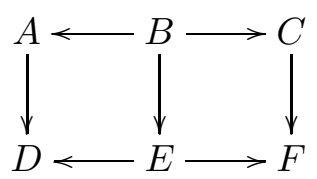

in the category of chain complexes, where all horizontal maps are monomorphisms and all vertical maps are quasi-isomorphisms, then the induced map from the pushout of the top row to the pushout of the bottom row is a quasi-isomorphism.

Proof of Lemma 6.7. Give the set of subcomplexes $L$ a total ordering such that if $L^{\prime} \subset L$ then $L^{\prime}<L$. We will prove each part by induction over this total order. So let $L$ be a subcomplex and suppose that all parts have been proved for all subcomplexes $<L$.

For (i), let $L^{\prime}$ be a subcomplex of $L$ and let $U$ be an open set of $L^{\prime}$. Let $\tau$ be a cell of $L$ of maximal dimension which is not in $L^{\prime}$, and let $M$ be the 
subcomplex of $L$ consisting of all cells except $\tau$. By inductive hypothesis, $U$ has a neighborhood $W$ in $X_{M}$ with the properties given in the statement of part (i). By Definition 2.6(c), $W \cap X_{\partial \tau}$ has a collar neighborhood $W_{1}$ for which the inclusion $W \cap X_{\partial \tau} \rightarrow W_{1}$ is a stratified deformation retract. Now let $V=W \cup W_{1}$; then $V$ is the desired neighborhood of $U$ in $X_{L}$.

For parts (ii), (iii) and (iv), let $\tau$ be a cell of $L$ of maximal dimension and let $L^{\prime}$ be the subcomplex of $L$ consisting of all cells except $\tau$.

For (ii), let $\xi \in I S_{i}^{\bar{n}}\left(X_{K} ; \mathbb{Z}\right) \cap \sum_{\sigma \in L} S_{i}\left(X_{\sigma} ; \mathbb{Z}\right)$. Write

$$
\xi=\sum a_{j} s_{j}
$$

where $a_{j} \in \mathbb{Z}$ and the $s_{j}$ are singular simplices. Let $U$ be the neighborhood of $X_{\tau}$ in $X_{L}$ given by part (i) and let $r: U \rightarrow X_{\tau}$ be the stratified retraction. Applying Proposition C.1 with the open set $U$ gives an intersection chain

$$
\bar{\xi}=\sum a_{j} \overline{s_{j}} .
$$

If we write

$$
\eta=\sum_{\operatorname{supp}\left(s_{j}\right) \subset X_{\tau}} a_{j} s_{j}
$$

then $\xi-\eta, \bar{\xi}-\eta$ and $r_{*}(\bar{\xi}-\eta)$ are all in in $\sum_{\sigma \in L^{\prime}} S_{*}\left(X_{\sigma} ; \mathbb{Z}\right)$. Now

$$
\xi=\left(\eta+r_{*}(\bar{\xi}-\eta)\right)+\left(\xi-\eta-r_{*}(\bar{\xi}-\eta)\right) .
$$

The first summand is in $I S_{*}^{\bar{n}}\left(X_{\tau} ; \mathbb{Z}\right)$ (because it is equal to $r_{*}(\bar{\xi})$ ), and the second is in $I S_{*}^{\bar{n}}\left(X_{K} ; \mathbb{Z}\right)$ (because $\xi$ and the first summand are) and in $\sum_{\sigma \in L^{\prime}} S_{*}\left(X_{\sigma} ; \mathbb{Z}\right)$, so by the inductive hypothesis $\xi$ is in $\sum_{\sigma \in L} I S_{i}^{\bar{n}}\left(X_{\sigma}, \mathbb{Z}\right)$ as required.

For (iii) and (iv), we use the fact that $\operatorname{colim}_{\sigma \in L} I S_{*}^{\bar{n}}\left(X_{\sigma} ; \mathbb{Z}\right)$ is the pushout of the diagram

$$
\underset{\sigma \in L^{\prime}}{\operatorname{colim}} I S_{*}^{\bar{n}}\left(X_{\sigma} ; \mathbb{Z}\right) \leftarrow \operatorname{colim}_{\sigma \in \partial \tau} I S_{*}^{\bar{n}}\left(X_{\sigma} ; \mathbb{Z}\right) \rightarrow I S_{*}^{\bar{n}}\left(X_{\tau} ; \mathbb{Z}\right) .
$$

For (iii), the map in question is obviously an epimorphism, so we only need to show that the map from the pushout of (6.2) to $I S_{*}^{\bar{n}}\left(X_{L} ; \mathbb{Z}\right)$ is a monomorphism. Using the inductive hypothesis, the pushout of (6.2) is the same as the pushout of

$$
\sum_{\sigma \in L^{\prime}} I S_{*}^{\bar{n}}\left(X_{\sigma} ; \mathbb{Z}\right) \leftarrow \sum_{\sigma \in \partial \tau} I S_{*}^{\bar{n}}\left(X_{\sigma} ; \mathbb{Z}\right) \rightarrow I S_{*}^{\bar{n}}\left(X_{\tau} ; \mathbb{Z}\right) .
$$

Suppose that $\xi \in \sum_{\sigma \in L^{\prime}} I S_{*}^{\bar{n}}\left(X_{\sigma} ; \mathbb{Z}\right)$ and $\eta \in I S_{*}^{\bar{n}}\left(X_{\tau} ; \mathbb{Z}\right)$ with

$$
\xi+\eta=0
$$

we need to show that $\xi \in \sum_{\sigma \in \partial \tau} I S_{*}^{\bar{n}}\left(X_{\sigma} ; \mathbb{Z}\right)$; by part (ii) it suffices to show that $\xi \in \sum_{\sigma \in \partial \tau} S_{*}\left(X_{\sigma} ; \mathbb{Z}\right)$. Equation (6.3) implies that each simplex $s$ of $\xi$ has its support in both $\tau$ and a cell of $L^{\prime}$; since $K$ is strict this implies that it has its support in a cell of $\partial \tau$ as required. 
Proof of (iv). By the inductive hypothesis and Remark 6.8, the map from the pushout of (6.2) to the pushout of

$$
I S_{*}^{\bar{n}}\left(X_{L^{\prime}} ; \mathbb{Z}\right) \leftarrow I S_{*}^{\bar{n}}\left(X_{\partial \tau} ; \mathbb{Z}\right) \rightarrow I S_{*}^{\bar{n}}\left(X_{\tau} ; \mathbb{Z}\right)
$$

is a quasi-isomorphism. By part (i) there is a neighborhood $U$ of $X_{L^{\prime}}$ in $X_{L}$ and a neighborhood $V$ of $X_{\tau}$ in $X_{L}$ such that the inclusions $X_{L^{\prime}} \rightarrow U$ and $X_{\tau} \rightarrow V$ are stratified deformation retractions. Then $X_{\partial \tau} \rightarrow U \cap V$ is a stratified deformation retraction, and hence the map from the pushout of (6.4) to the pushout of

$$
I S_{*}^{\bar{n}}(U ; \mathbb{Z}) \leftarrow I S_{*}^{\bar{n}}(U \cap V ; \mathbb{Z}) \rightarrow I S_{*}^{\bar{n}}(V ; \mathbb{Z})
$$

is a quasi-isomorphism. Finally, the map from the pushout of (6.5) to $I S_{*}^{\bar{n}}\left(X_{L} ; \mathbb{Z}\right)$ is a quasi-isomorphism by [FM13b, Proposition 6.3] and [F07, Proposition 2.9].

\section{L-THEORY OF THE FUNDAMENTAL GROUPOID.}

Let $Z$ be a path-connected space.

For our further work, we need to use the fundamental groupoid $\pi_{1} Z$ rather than the fundamental group $\pi_{1}\left(Z, z_{0}\right)$. The main reason is that we will be using the results of [WW95], and these require a functor defined on unbased spaces; cf. lines 9-12 of [WW95, Subsection 2.1]. An additional benefit is that we won't need to choose basepoints.

In this section we define $L$-spectra and $L$-groups over $\pi_{1} Z$. We will make use of the definitions in Appendix B.

First we define an additive category $\mathbb{Z}\left[\pi_{1} Z\right]$ by letting the objects be the points of $Z$ and letting the abelian group of morphisms from $z$ to $z^{\prime}$ be the free abelian group generated by the morphisms from $z$ to $z^{\prime}$ in the groupoid. The composition of morphisms is similar to the multiplication in a group ring.

For a module $\mathcal{M}$ we write $\mathcal{M}_{z}$ for the restriction of $\mathcal{M}$ to the full subcategory $\mathbb{Z}\left[\pi_{1}(Z, z)\right]$ with only one object $z$; this is a left module (in the usual sense) over the ring $\mathbb{Z}\left[\pi_{1}(Z, z)\right]$, and $\mathcal{M}$ is determined up to unique isomorphism by $\mathcal{M}_{z}$.

Remark 7.1. The evident map $\mathcal{M}_{z} \otimes_{\mathbb{Z}\left[\pi_{1}(Z, z)\right]} \mathcal{N}_{z} \rightarrow \mathcal{M} \otimes_{\mathbb{Z}\left[\pi_{1} Z\right]} \mathcal{N}$ is an isomorphism for any $z$ (because there is a map in the other direction which is inverse to it).

For left modules $\mathcal{M}$ and $\mathcal{M}^{\prime}$, we define $\operatorname{Hom}_{\mathbb{Z}\left[\pi_{1} Z\right]}\left(\mathcal{M}, \mathcal{M}^{\prime}\right)$ to be the abelian group of natural transformations.

Remark 7.2. The restriction map

$$
\operatorname{Hom}_{\mathbb{Z}\left[\pi_{1} Z\right]}\left(\mathcal{M}, \mathcal{M}^{\prime}\right) \rightarrow \operatorname{Hom}_{\mathbb{Z}\left[\pi_{1}(Z, z)\right]}\left(\mathcal{M}_{z}, \mathcal{M}_{z}^{\prime}\right)
$$

is an isomorphism for all $z$ (because there is a map in the other direction which is inverse to it). 
$\mathcal{M}$ is free if $\mathcal{M}_{z}$ is a free $\mathbb{Z}\left[\pi_{1}(Z, z)\right]$-module for some (and hence for all) $z$.

The category of left $\mathbb{Z}\left[\pi_{1} Z\right]$ modules is an abelian category, so the concepts of chain complex, chain homotopy, and quasi-isomorphism can be defined in the usual way.

Remark 7.3. Note that two chain complexes are chain homotopy equivalent (resp., quasi-isomorphic) over $\mathbb{Z}\left[\pi_{1} Z\right]$ if and only if their restrictions to $\mathbb{Z}\left[\pi_{1}(Z, z)\right]$ are chain homotopy equivalent (resp., quasi-isomorphic) over $\mathbb{Z}\left[\pi_{1}(Z, z)\right]$ for some $z$.

The involution of $\mathbb{Z}\left[\pi_{1} Z\right]$ is the additive functor

$$
\mathbb{Z}\left[\pi_{1} Z\right] \rightarrow \mathbb{Z}\left[\pi_{1} Z\right]^{\text {op }}
$$

which takes a morphism $g: z \rightarrow z^{\prime}$ in the groupoid to $g^{-1}: z^{\prime} \rightarrow z$. This restricts to the usual involution of $\mathbb{Z}\left[\pi_{1}(Z, z)\right]$.

With these definitions, it is straightforward to generalize the definitions and results of [LM13, Section 9] and [LM, Sections 12-14] with the ring $R$ replaced by $\mathbb{Z}\left[\pi_{1} Z\right]$, with these modifications:

- For the analogue of [LM13, Definition 9.9(b)], the left $R$-module $R$ should be replaced by any left $\mathbb{Z}\left[\pi_{1} Z\right]$ module of the form $\operatorname{Hom}(z,-)$.

- For the proof of the analogue of [LM13, Theorem 9.11], note that a pre $K$-ad $G$ with values in $\mathcal{A}^{\mathbb{Z}\left[\pi_{1} Z\right]}$ determines, for each $z \in Z$, a pre $K$-ad $G_{z}$ with values in $\mathcal{A}^{\mathbb{Z}\left[\pi_{1}(Z, z)\right]}$, and that (using Remarks 7.1 and 7.2) $G$ is an ad if and only if each $G_{z}$ is.

In particular, we obtain an ad theory $\operatorname{ad}^{\mathbb{Z}\left[\pi_{1} Z\right]}$, bordism groups $\Omega_{*}^{\mathbb{Z}\left[\pi_{1} Z\right]}$, and spectra $\mathbf{Q}^{\mathbb{Z}\left[\pi_{1} Z\right]}$ and $\mathbf{Q}_{\geq 0}^{\mathbb{Z}\left[\pi_{1} Z\right]}$. In analogy with [Ran92, page 60] we define $L^{n}\left(\mathbb{Z}\left[\pi_{1} Z\right]\right)$ to be $\Omega_{n}^{\mathbb{Z}\left[\pi_{1} Z\right]}$ for $n \geq 0$ and 0 for $n<0$; by Remark 3.1. $L^{n}\left(\mathbb{Z}\left[\pi_{1} Z\right]\right) \cong \pi_{n} \mathbf{Q}_{\geq 0}^{\mathbb{Z}\left[\pi_{1} Z\right]}$ (cf. Remark 1.1) .

Remark 7.4. Let $z \in Z$. From what has been said it is clear that the forgetful functor from $\mathbb{Z}\left[\pi_{1} Z\right]$ modules to $\mathbb{Z}\left[\pi_{1}(Z, z)\right]$ modules induces an isomorphism

$$
L^{n}\left(\mathbb{Z}\left[\pi_{1} Z\right]\right) \rightarrow L^{n}\left(\mathbb{Z}\left[\pi_{1}(Z, z)\right]\right) .
$$

Remark 7.5. For use in the next section we observe that if $\mathcal{M}$ and $\mathcal{M}^{\prime}$ are left $\mathbb{Z}\left[\pi_{1} Z\right]$ modules we can define a tensor product $\mathcal{M} \otimes \mathcal{M}^{\prime}$ to be the left $\mathbb{Z}\left[\pi_{1} Z\right]$ module which takes $z$ to $\mathcal{M}_{z} \otimes \mathcal{M}_{z}^{\prime}$, with the evident action of the morphisms. If $\mathcal{Z}$ is the constant right module with value $\mathbb{Z}$ then the isomorphism of left $Z\left[\pi_{1} Z\right]$ modules

$$
\mathcal{Z}^{t} \otimes \mathcal{M} \otimes \mathcal{M}^{\prime} \cong \mathcal{M} \otimes \mathcal{M}^{\prime}
$$

induces an isomorphism

$$
\mathcal{Z} \otimes_{\mathbb{Z}\left[\pi_{1} Z\right]}\left(\mathcal{M} \otimes \mathcal{M}^{\prime}\right) \cong \mathcal{M}^{t} \otimes_{\mathbb{Z}\left[\pi_{1} Z\right]} \mathcal{M}^{\prime}
$$


Remark 7.6. For use in Section $\left[10\right.$, we give a variant of $\operatorname{ad}^{\mathbb{Z}\left[\pi_{1} Z\right]}$. Let $\mathcal{A}_{\text {fin }}^{\mathbb{Z}\left[\pi_{1} Z\right]}$ be the full subcategory of $\mathcal{A}^{\mathbb{Z}\left[\pi_{1} Z\right]}$ consisting of objects $(C, \varphi)$ for which $C$ is finite (not just homotopy finite). For a ball complex $K$, let $\operatorname{ad}_{\text {fin }}^{\mathbb{Z}\left[\pi_{1} Z\right]}(K)$ be the set of pre $K$-ads with values in $\mathcal{A}_{\text {fin }}^{\mathbb{Z}\left[\pi_{1} Z\right]}$ for which the composite with the forgetful functor to $\mathcal{A}^{\mathbb{Z}\left[\pi_{1} Z\right]}$ is an ad. The proof of [LM13, Theorem 9.11] generalizes to show that $\operatorname{ad}_{\text {fin }}^{\mathbb{Z}\left[\pi_{1} Z\right]}$ is an ad theory, and the proof of [LM, Proposition B.17] (specifically, the proof that $\Omega_{*}^{c}$ is an isomorphism) shows that the map $\operatorname{ad}_{\mathrm{fin}}^{\mathbb{Z}\left[\pi_{1} Z\right]} \rightarrow \operatorname{ad}^{\mathbb{Z}\left[\pi_{1} Z\right]}$ induces an isomorphism of bordism groups.

\section{The SYMMETRIC SIGNATURE OF AN IP-SPACE}

For a compact oriented $n$-manifold $M$ (and more generally for a Poincaré duality space) the symmetric signature $\sigma^{*}(M)$ is an element of the symmetric $L$-group $L^{n}\left(\mathbb{Z}\left[\pi_{1} M\right]\right)$. The symmetric signature was introduced by Miščenkd 4 as a tool for studying the Novikov conjecture, and since then it has become an important part of surgery theory (see [Ran92], for example). The symmetric signature has many useful properties, such as homotopy invariance, bordism invariance, and a product formula.

The paper [CSW91] has a brief description of a construction (using controlled topology) which assigns to a compact oriented Witt space $X$ and a point $x \in X$ a symmetric signature in $L^{n}\left(\mathbb{Q}\left[\pi_{1}(X, x)\right]\right)$, with properties analogous to those of Miščenko's symmetric signature (further information about this construction is given in [W94, pages 209-210]). A simpler construction with the same properties was given in [FM13b, Subsection 5.4]. The two constructions are known to agree rationally by an argument due to Weinberger (cf. [ALMP12, Proposition 11.1]) and, independently, BanaglCappell-Shaneson [BCS03, Proposition 2].

In this section we show that when $X$ is a connected compact oriented IP-space of dimension $n$ the method used in FM13b gives a symmetric signature

$$
\sigma_{\mathrm{IP}}^{*}(X) \in L^{n}\left(\mathbb{Z}\left[\pi_{1} X\right]\right)
$$

with the usual properties.

Remark 8.1. Recall ([LM, Section 12]) that the relaxed symmetric Poincaré ad theory $\operatorname{ad}_{\text {rel }}^{\mathbb{Z}}$ described in Subsection 5.2 has an analog $\operatorname{ad}_{\text {rel }}^{R}$ when $R$ is any ring with involution. The same construction gives an ad theory $\operatorname{ad}_{\mathrm{rel}}^{\mathbb{Z}\left[\pi_{1} X\right]}$ 齿

\footnotetext{
${ }^{4}$ Miščenko's construction gives an element of $L^{n}\left(\mathbb{Z}\left[\pi_{1}(X, x)\right]\right)$ for each $x$, and these are consistent as $x$ varies, so by Remark 7.4 one obtains a well-defined element of $L^{n}\left(\mathbb{Z}\left[\pi_{1} X\right]\right)$.

${ }^{5}$ In [LM13, Section 9] it was assumed that for any object $(C, \varphi)$ the chain complex $C$ is free over $R$ (this assumption was built into the definition of the category $\mathcal{D}$ LM13, Definition $9.2(\mathrm{v})]$ ). This was for two reasons: to ensure that the $L$ groups would be the same as in [Ran92, but it is not needed for this, see [LM Remark B.18]; and for functoriality ([LM13, Section 13]), but it is not needed for this, see [LM, Subsection B.7]. The same category $\mathcal{D}$ was used in [LM, Definition 12.1], but freeness is not needed there
} 
The construction in LM13, Example 12.2(i)] generalizes to give a map

$$
\operatorname{ad}^{\mathbb{Z}\left[\pi_{1} X\right]} \rightarrow \operatorname{ad}_{\mathrm{rel}}^{\mathbb{Z}\left[\pi_{1} X\right]}
$$

and the proof of [LM, Proposition 13.3] generalizes to show that this induces an isomorphism of bordism groups. Thus we have an isomorphism

$$
L^{n}\left(\mathbb{Z}\left[\pi_{1} X\right]\right)=\left(\Omega^{\mathbb{Z}\left[\pi_{1} X\right]}\right)_{n} \cong\left(\Omega_{\mathrm{rel}}^{\mathbb{Z}\left[\pi_{1} X\right]}\right)_{n}
$$

for $n \geq 0$,

Because of the isomorphism (8.1), we can construct $\sigma_{\mathrm{IP}}^{*}(X)$ by giving a suitable element of $\left(\Omega_{\mathrm{rel}}^{\mathbb{Z}\left[\pi_{1} X\right]}\right)_{n}$. For this in turn it suffices (by [LM13, Definition 4.2]) to construct an element of $\operatorname{ad}_{\text {rel }}^{\mathbb{Z}\left[\pi_{1} X\right]}(*)$, which we do as follows. For each $x \in X$ let $\tilde{X}_{x}$ be the universal cover constructed in the usual way as equivalence classes of paths starting at $x$. Let $A_{X}$ be the chain complex of left $\mathbb{Z}\left[\pi_{1} X\right]$ modules with

$$
\left(A_{X}\right)_{x}=I S_{*}^{\overline{0}}\left(\tilde{X}_{x} ; \mathbb{Z}\right),
$$

and let $\mathcal{Z}$ be the constant left $\mathbb{Z}\left[\pi_{1} X\right]$ module with value $\mathbb{Z}$. Choose a cycle $\xi \in \mathcal{Z} \otimes_{\mathbb{Z}\left[\pi_{1} X\right]} A_{X}$ which maps to a representative for the fundamental class $\Gamma_{X} \in I H_{n}^{\overline{0}}(X ; \mathbb{Z})$; this is always possible by [FM13b, Proposition 6.1.3].

Definition 8.2. Let $\left(C_{X}, D_{X}, \beta_{X}, \varphi\right)$ be defined as follows.

- $C_{X}$ is the chain complex of $\mathbb{Z}\left[\pi_{1} X\right]$ modules with $\left(C_{X}\right)_{x}=I S_{*}^{\bar{n}}\left(\tilde{X}_{x} ; \mathbb{Z}\right)$.

- Let $E_{X}$ be the chain complex of left $\mathbb{Z}\left[\pi_{1} X\right]$ modules with

$$
\left(E_{X}\right)_{x}=I S_{*}^{Q \bar{n}, \bar{n}}\left(\tilde{X}_{x} \times \tilde{X}_{x} ; \mathbb{Z}\right),
$$

where $\mathbb{Z}\left[\pi_{1}(X, x)\right]$ acts diagonally; then $D_{X}$ is the chain complex of abelian groups

$$
\mathcal{Z} \otimes_{\mathbb{Z}\left[\pi_{1} X\right]} E_{X}
$$

with the evident $\mathbb{Z} / 2$ action.

- $\beta_{X}$ is the map

$$
\left(C_{X}\right)^{t} \otimes_{\mathbb{Z}\left[\pi_{1} X\right]} C_{X} \rightarrow D_{X}
$$

determined by the composites

$$
\begin{gathered}
I S_{*}^{\bar{n}}\left(\tilde{X}_{x} ; \mathbb{Z}\right)^{t} \otimes_{\mathbb{Z}\left[\pi_{1}(X, x)\right]} I S_{*}^{\bar{n}}\left(\tilde{X}_{x} ; \mathbb{Z}\right) \cong \mathbb{Z} \otimes_{\mathbb{Z}\left[\pi_{1}(X, x)\right]}\left(I S_{*}^{\bar{n}}\left(\tilde{X}_{x} ; \mathbb{Z}\right) \otimes I S_{*}^{\bar{n}}\left(\tilde{X}_{x} ; \mathbb{Z}\right)\right) \\
\left.\stackrel{1 \otimes \times}{\longrightarrow} \mathbb{Z} \otimes_{\mathbb{Z}\left[\pi_{1}(X, x)\right]} I S_{*}^{Q \bar{n}, \bar{n}}\left(\tilde{X}_{x} \times \tilde{X}_{x}\right) ; \mathbb{Z}\right),
\end{gathered}
$$

where the first isomorphism is given by Remark 7.5.

- $\varphi \in\left(D_{X}\right)^{\mathbb{Z} / 2}$ is the image of $\xi$ under the map $\mathcal{Z} \otimes_{\mathbb{Z}\left[\pi_{1} X\right]} A_{X} \rightarrow \mathcal{Z} \otimes_{\mathbb{Z}\left[\pi_{1} X\right]}$ $E_{X}$ induced by the diagonal maps

$$
I S_{n}^{\overline{0}}\left(\tilde{X}_{x} ; \mathbb{Z}\right) \rightarrow I S_{n}^{Q \bar{n}, \bar{n}}\left(\tilde{X}_{x} \times \tilde{X}_{x} ; \mathbb{Z}\right) .
$$

Lemma 8.3. $\left(C_{X}, D_{X}, \beta_{X}, \varphi\right)$ is an element of $\operatorname{ad}_{\mathrm{rel}}^{\mathbb{Z}\left[\pi_{1} X\right]}(*)$.

either. We shall therefore assume that for objects $(C, D, \beta, \varphi)$ in $\mathcal{A}_{\mathrm{rel}}^{\mathbb{Z}\left[\pi_{1} X\right]}$ the chain complex $C$ is homotopy finite but not necessarily free. 
Proof. First we need to show that $C_{X}$ is homotopy finite. Fix $x \in X$. Proposition D.8 gives a quasi-isomorphism over $\mathbb{Z}\left[\pi_{1}(X, x)\right]$ from $\left(C_{X}\right)_{x}$ to a finite chain complex $A$ over $\mathbb{Z}\left[\pi_{1}(X, x)\right] .\left(C_{X}\right)_{x}$ is chain homotopy equivalent to a free complex over $\mathbb{Z}\left[\pi_{1}(X, x)\right]$ by Proposition D.3(i), and HS71, Exercise IV.4.2] says that a quasi-isomorphism between free complexes is a chain homotopy equivalence, so the quasi-isomorphism $\left(C_{X}\right)_{x} \rightarrow A$ is a chain homotopy equivalence over $\mathbb{Z}\left[\pi_{1}(X, x)\right]$. Now $A$ extends to a finite chain complex $B$ over $\mathbb{Z}\left[\pi_{1} X\right]$, and the chain homotopy equivalence $\left(C_{X}\right)_{x} \rightarrow A$ extends to a chain homotopy equivalence $C_{X} \rightarrow B$ over $\mathbb{Z}\left[\pi_{1} X\right]$.

$\beta_{X}$ is a quasi-isomorphism by Proposition D.6 and Remark D.5.

It remains to show that the slant product with $\left(\beta_{*}\right)^{-1}([\varphi])$ is an isomorphism. For this we observe that the proof of [FM13b, Proposition 5.17] goes through with $\mathbb{Z}$ coefficients instead of $F$ coefficients if we use Theorem E.3 in place of [FM13b, Theorem 4.1]. Inspection of the definitions in [FM13b, Subsection 5.4] (using the fact that the map denoted $\Upsilon$ there is the same as $\beta_{*}$ ) shows that (after replacing $F$ coefficients by $\mathbb{Z}$ coefficients) the element denoted in the proof of FM13b, Proposition 5.17] by $\psi_{*}(\iota)$ is the same as $\left(\beta_{*}\right)^{-1}([\varphi])$, so Lemma 8.3 follows from the $\mathbb{Z}$-version of [FM13b, Proposition 5.17].

Next observe that the bordism class of $\left(C_{X}, D_{X}, \beta_{X}, \varphi\right)$ is independent of the choice of $\xi$ by [FM13b, Remark 5.6].

Definition 8.4. Let $X$ be a compact oriented IP-space of dimension $n$. Then $\sigma_{\mathrm{IP}}^{*}(X) \in L^{n}\left(\mathbb{Z}\left[\pi_{1} X\right]\right)$ is the element which maps to the bordism class of $\left(C_{X}, D_{X}, \beta_{X}, \varphi\right)$ under the isomorphism (8.1).

Remark 8.5. The properties of the symmetric signature given in FM13b, Subsection 5.5] remain valid (with $\mathbb{Z}$ coefficients instead of $F$ coefficients) for $\sigma_{\text {IP }}^{*}$, with the same proofs, provided that Proposition D.3(i), Theorem E.3. and Theorem E.5 are used instead of FM13b, Proposition 5.15, Theorem 4.1, Theorem 4.5]. For example, Prop. 5.20 of [FM13b] asserts that if $n$ is divisible by 4 , then the composition

$$
L^{n}(\mathbb{Q}[G]) \longrightarrow L^{n}(\mathbb{Q}) \stackrel{\cong}{\longrightarrow} W(\mathbb{Q}),
$$

where $W(\mathbb{Q})$ is the Witt group of $\mathbb{Q}$, takes the symmetric signature of a Witt space $X$ with $\pi_{1}(X)=G$ to the Witt class of the intersection form on the middle dimensional, middle perversity intersection homology of $X$. For IP-spaces $X$, the analogous statement is: If $n$ is divisible by 4 , then the composition

$$
L^{n}(\mathbb{Z}[G]) \longrightarrow L^{n}(\mathbb{Z}) \stackrel{\cong}{\longrightarrow} \mathbb{Z}
$$

takes $\sigma_{\mathrm{IP}}^{*}(X)$ to the ordinary signature of the intersection form of $X$.

Remark 8.6. We have only described the universal symmetric signature of IP-spaces. More generally, one can similarly construct the symmetric signature $\sigma_{\mathrm{IP}}^{*}(r) \in L^{n}(\mathbb{Z}[G])$ of any reference map $r: X \rightarrow B G$ with $X$ IP and $G$ any discrete group. Instead of using the intersection chains of the universal 
cover of $X$, one uses the intersection chains of the cover of $X$ induced by $r$. This is done in [FM13b] for global Witt maps $r$. For such maps, [FM13b, Thm. 5.23] asserts that $\sigma_{\mathrm{Witt}}^{*}(r \circ f)=\sigma_{\mathrm{Witt}}^{*}(r)$ for orientation preserving stratified homotopy equivalences $f: X^{\prime} \rightarrow X$ between Witt spaces. Similarly for orientation preserving stratified homotopy equivalences $f$ between IP-spaces,

$$
\sigma_{\mathrm{IP}}^{*}(r \circ f)=\sigma_{\mathrm{IP}}^{*}(r) .
$$

\section{The $L$-Theory Fundamental Class}

For an $n$-dimensional compact oriented topological manifold $M$, Ranicki constructs an $L$-theory fundamental class $[M]_{\mathbb{L}} \in \mathrm{L}_{n}^{\bullet}(M)$ ([Ran92, Section 16]) which plays an important role in surgery theory. It is an oriented homeomorphism invariant whose image under the assembly map

$$
\mathrm{L}_{n}^{\bullet}(M) \rightarrow L^{n}\left(\mathbb{Z}\left[\pi_{1} M\right]\right)
$$

is the symmetric signature $\sigma^{*}(M) .[M]_{\mathbb{L}}$ can be constructed in the following way. There is an equivalence

$$
M \text { STop } \rightarrow \mathbf{Q}_{\mathrm{STop}}
$$

in the stable category, where $M \mathrm{ST}$ Top is the Thom spectrum and $\mathbf{Q}_{\mathrm{STop}}$ is the Quinn spectrum; see [LM13, Appendix B] for details. There is an ad theory $\operatorname{ad}_{\text {STopFun }}$ which is related to $\operatorname{ad}_{\text {STop }}$ in the same way that $\operatorname{ad}_{\text {IPFun }}$ is related to $\operatorname{ad}_{\mathrm{IP}}$ (see the end of [LM13, Section 8]), and the map given by forgetting the chain representative is an equivalence

$$
\mathbf{Q}_{\text {STopFun }} \stackrel{\simeq}{\rightarrow} \mathbf{Q}_{\text {STop }} \text {. }
$$

The symmetric signature gives a map 6

$$
\operatorname{sig}: \mathbf{Q}_{\mathrm{STopFun}} \rightarrow \mathbf{Q}_{\geq 0}^{\mathbb{Z}}=\mathbb{L} \bullet(\mathbb{Z}),
$$

so we have a map in the stable category

$$
\text { Sig : } \mathbf{Q}_{\text {STop }} \rightarrow \mathbb{L} \bullet(\mathbb{Z}) \text {. }
$$

Now if $M$ is an $n$-dimensional compact oriented topological manifold then the identity map $M \rightarrow M$ represents an element in $\left(\Omega_{\mathrm{STop}}\right)_{n}(M)$. Let $[M]_{\mathrm{STop}}$ denote the image of this element under the isomorphism $\left(\Omega_{\mathrm{STop}}\right)_{n}(M) \cong \operatorname{MSTop}_{n}(M)$. We have

Lemma 9.1. The image of $[M]_{\mathrm{STop}}$ under the composite

$$
M \operatorname{STop}_{n}(M) \rightarrow\left(\mathbf{Q}_{\mathrm{STop}}\right)_{n}(M) \stackrel{\operatorname{Sig}}{\longrightarrow} \mathbb{L} \bullet(\mathbb{Z})_{n}(M)=\mathrm{L}_{n}^{\bullet}(M)
$$

is $[M]_{\mathbb{L}}$.

\footnotetext{
${ }^{6}$ Specifically, the end of [LM13, Section 8] gives a morphism $\mathcal{A}_{\text {StopFun }} \rightarrow \mathcal{A}_{e, *, 1}$, LM13, Section 10] gives a morphism sig : $\mathcal{A}_{e, *, 1} \rightarrow \mathcal{A}^{\mathbb{Z}}$, (which was denoted Sig in [LM13]) and the map (9.1) is, by definition, induced by the the composite of these two morphisms.
} 
Proof. The construction of $[M]_{\mathbb{L}}$ is given in $[$ Ran92, Propositions 16.15 and 16.16(ii)]. The class $[M]_{\mathbb{L}}$ is denoted by $\operatorname{sign}_{M}^{\mathbf{L}^{\bullet}}(M)$ in KMM13, Definition 8.11], and [KMM13, Proposition 13.3] says that it is $S$-dual to the orientation class $u_{\mathbf{L}} \cdot(\nu) \in \mathbb{L} \bullet(\mathbb{Z})^{k}(T(\nu))$ (where $T(\nu)$ is the Thom complex of the normal bundle and $k$ is the dimension of $\nu$ ). By the proof of [KMM13, Proposition 13.2], $u_{\mathbf{L}} \cdot(\nu)$ is obtained by applying the composite

$$
M \text { STop } \rightarrow \mathbf{Q}_{\text {STop }} \stackrel{\text { Sig }}{\longrightarrow} \mathbb{L}^{\bullet}(\mathbb{Z})
$$

to the orientation class $u_{M \mathrm{STop}}(\nu) \in M \mathrm{STop}^{k}(T(\nu))$ represented by the map

$$
T(\nu) \rightarrow \mathrm{STop}_{k}
$$

Thus it suffices to show that the $S$-dual of $u_{M \text { STop }}(\nu)$ is the element we have called $[M]_{\mathrm{STop}}$. The $S$-dual of $u_{M \mathrm{STop}}(\nu)$ is represented by the composite

$$
S^{n+k} \stackrel{p}{\rightarrow} T(\nu) \stackrel{\delta}{\rightarrow} T(\nu) \wedge M_{+} \rightarrow \operatorname{STop}_{k} \wedge M_{+},
$$

where $p$ is the Pontrjagin-Thom collapse and $\delta$ is the Thom diagonal (see for example [Rezk13, Proposition 2.2], with the maps $\eta$ and $\phi$ given on page 7 of [Rezk13]) and by [St68, page 19 and Example 6 on page 43] this composite represents $[M]_{\text {STop }}$.

Remark 9.2. The composite

$$
\text { MSTop } \rightarrow \mathbf{Q}_{\text {STop }} \stackrel{\text { Sig }}{\longrightarrow} \mathbb{L}^{\bullet}(\mathbb{Z})
$$

is the Sullivan-Ranicki orientation (compare [LM, Remark 1.4]).

Our goal in this section is to prove

Theorem 9.3. For an $n$-dimensional compact oriented IP-space $X$ there is a fundamental class $[X]_{\mathbb{L}} \in \mathrm{L}_{n}^{\bullet}(X)$ with the following properties:

(i) $[X]_{\mathbb{L}}$ is an oriented $P L$ homeomorphism invariant.

(ii) If $X$ is a PL manifold then $[X]_{\mathbb{L}}$ is the same as the fundamental class constructed by Ranicki.

(We will show in the next section that $[X]_{\mathbb{L}}$ assembles to the symmetric signature $\sigma_{\mathrm{IP}}^{*}(X)$ given by Definition 8.4 .)

Remark 9.4. For Witt spaces, a different method for constructing a fundamental class is described in CSW91.

The rest of the section is devoted to the proof of Theorem 9.3. We begin with the construction of $[X]_{\mathbb{L}}$.

Define a category $\mathcal{A}_{\mathrm{IP}, Z}$ as follows. An object of $\mathcal{A}_{\mathrm{IP}, Z}$ is an object $X$ of $\mathcal{A}_{\text {IP }}$ together with a map of topological spaces $X \rightarrow Z$. A morphism from $X \rightarrow Z$ to $X^{\prime} \rightarrow Z$ is a morphism $X \rightarrow X^{\prime}$ in $\mathcal{A}_{\text {IP }}$ for which the diagram

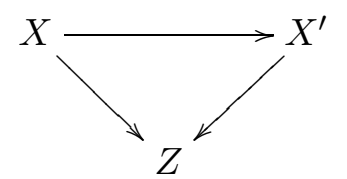


commutes. There is a forgetful functor

$$
\Upsilon: \mathcal{A}_{\mathrm{IP}, Z} \rightarrow \mathcal{A}_{\mathrm{IP}}
$$

and we define $\operatorname{ad}_{\mathrm{IP}, Z}^{k}(K) \subset \operatorname{pre}_{\mathrm{IP}, Z}^{k}(K)$ to be the set of functors $F$ for which the composite $\Upsilon \circ F$ is in $\operatorname{ad}_{\mathrm{IP}}^{k}(K)$. The proof of Theorem 4.4 shows that this is an ad theory.

Now we have a functor $\Phi$ from spaces to spectra with

$$
\Phi(Z)=\mathrm{Q}_{\mathrm{IP}, Z}
$$

By Remark 3.1, the homotopy groups of $\mathbf{Q}_{\mathrm{IP}, Z}$ are the same as the IP bordism groups $\left(\Omega_{\mathrm{IP}}\right)_{*}(Z)$ defined by Pardon ([Par90, Section 5$\left.]\right)$. Pardon proves that $\left(\Omega_{\mathrm{IP}}\right)_{*}$ is a homology theory, and in particular this shows that $\Phi$ is homotopy invariant in the sense of [WW95, Section 1]. We therefore have an assembly map

$$
\alpha: Z_{+} \wedge \Phi(*) \rightarrow \Phi(Z)
$$

by [WW95, Theorem 1.1 and Observation 1.2] for all spaces $Z$ which have the homotopy type of a CW complex.

Theorem 9.5. $\alpha$ is a weak equivalence.

The proof is deferred to Section 14 , Now we can define $[X]_{\mathbb{L}}$.

Definition 9.6. Let $X$ be an $n$-dimensional compact oriented IP-space.

(i) Let $[X]_{\mathrm{IP}} \in \pi_{n}\left(\mathbf{Q}_{\mathrm{IP}, X}\right)$ be the image of the class of the identity map $X \rightarrow X$ in $\left(\Omega_{\mathrm{IP}}\right)_{n}(X)$ under the isomorphism $\left(\Omega_{\mathrm{IP}}\right)_{n}(X) \cong \pi_{n}\left(\mathbf{Q}_{\mathrm{IP}, X}\right)$.

(ii) Let $[X]_{\mathbb{L}}$ be the image of $[X]_{\mathrm{IP}}$ under the composite

$$
\pi_{n}\left(\mathbf{Q}_{\mathrm{IP}, X}\right) \stackrel{\alpha}{\stackrel{\alpha}{\cong}}\left(\mathbf{Q}_{\mathrm{IP}}\right)_{n}(X) \stackrel{\operatorname{Sig}}{\longrightarrow}\left(\mathbf{Q}_{\mathrm{rel}, \geq 0}^{\mathbb{Z}}\right)_{n}(X) \stackrel{\cong}{\bullet} \mathrm{L}_{n}^{\bullet}(X),
$$

where the last map is the isomorphism (8.1).

It remains to prove parts (i) and (ii) of Theorem 9.3. For part (i) it suffices to show that if $f: X \rightarrow X^{\prime}$ is an oriented PL homeomorphism then $f_{*}\left([X]_{\mathrm{IP}}\right)=\left[X^{\prime}\right]_{\mathrm{IP}}$, and this in turn follows from the fact that the map

$$
(I \times X) \cup_{1 \times X} \quad X^{\prime} \rightarrow X^{\prime},
$$

which is the identity on $X^{\prime}$ and takes $(t, x)$ to $f(x)$, is a bordism between $f$ and the identity map of $X^{\prime}$.

For part (ii), we need to compare the composites (9.2) and (9.3) for $X$ a PL manifold $M$. [LM, Section 14] gives a morphism

$$
\operatorname{sig}_{\text {rel }}: \mathcal{A}_{e, *, 1} \rightarrow \mathcal{A}_{\text {rel }}^{\mathbb{Z}}
$$

and as in Footnote 6 (with $\mathcal{A}_{\text {rel }}^{\mathbb{Z}}$ instead of $\mathcal{A}^{\mathbb{Z}}$ ) this gives a map

$$
\operatorname{sig}: \mathbf{Q}_{\mathrm{STopFun}} \rightarrow \mathbf{Q}_{\mathrm{rel}, \geq 0}^{\mathbb{Z}}
$$

which in turn gives

$$
\operatorname{Sig}: \mathbf{Q}_{\mathrm{STop}} \rightarrow \mathbf{Q}_{\mathrm{rel}, \geq 0}^{\mathbb{Z}}
$$


By [LM, Proposition 14.4], (9.2) is equal to the composite

$$
M \operatorname{STop}_{n}(M) \rightarrow\left(\mathbf{Q}_{\mathrm{STop}}\right)_{n}(M) \stackrel{\mathrm{Sig}}{\longrightarrow}\left(\mathbf{Q}_{\mathrm{rel}, \geq 0}^{\mathbb{Z}}\right)_{n}(M) \stackrel{\cong}{\bullet} \mathrm{L}_{n}^{\bullet}(M) .
$$

Next we observe that for each space $Z$ there is an ad theory $\operatorname{ad}_{\mathrm{STop}, Z}$ defined analogously to $\operatorname{ad}_{\mathrm{IP}, Z}$. We get a functor $\Xi$ from spaces to spectra by letting

$$
\Xi(Z)=\mathbf{Q}_{\mathrm{STop}, Z},
$$

and we have

$$
\pi_{*} \Xi(Z) \cong\left(\Omega_{\mathrm{STop}}\right)_{*}(Z) .
$$

$\Xi$ is homotopy invariant, because $\pi_{*} \Xi(Z)$ is a homology theory by C79, Chapter 4], and the proof of Theorem 9.5 shows that the assembly map for $\Xi$ is a weak equivalence.

Lemma 9.7. The composite (9.4) is equal to the composite

$$
\pi_{n} \mathbf{Q}_{\mathrm{STop}, M} \stackrel{\alpha}{\leftarrow}\left(\mathbf{Q}_{\mathrm{STop}}\right)_{n}(M) \stackrel{\operatorname{Sig}}{\longrightarrow}\left(\mathbf{Q}_{\mathrm{rel}, \geq 0}^{\mathbb{Z}}\right)_{n}(M) \stackrel{\cong}{\longleftarrow} \mathbf{L}_{n}^{\bullet}(M)
$$

where $\alpha$ is the assembly map for the functor $\Xi$.

We defer the proof for a moment. Next we observe that everything we have said about topological manifolds and STop is also valid for PL manifolds and SPL. We write $\Xi^{\prime}$ for the PL analog of $\Xi$. Now consider the following diagram.

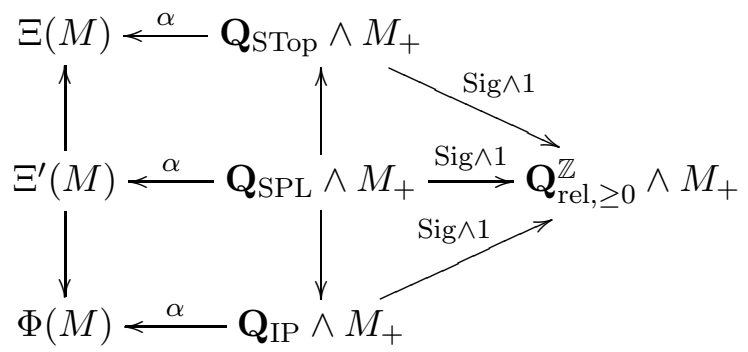

The squares commute up to homotopy by the naturality of the assembly map, and the triangles commute by the definition of the maps Sig. The upper left-hand vertical map takes $[M]_{\mathrm{PL}}$ to $[M]_{\mathrm{STop}}$, and (by Lemma 9.7) the upper composite takes $[M]_{\text {STop }}$ to the fundamental class constructed by Ranicki. The lower left-hand vertical map takes $[M]_{\mathrm{PL}}$ to $[M]_{\mathrm{IP}}$, and the lower composite takes $[M]_{\mathrm{STop}}$ to the fundamental class given by Definition 9.6(ii).

Proof of Lemma 9.7. It suffices to show that the diagram

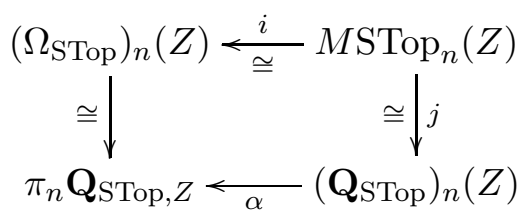


commutes, where $Z$ is a space, $i$ is the standard isomorphism and $j$ is given by [LM13, Appendix B] 7

First we recall the definition of $i$ (cf. DK01, pages 224-5]). The $k$-th space of the spectrum $M \mathrm{STop} \wedge Z_{+}$is $T \mathrm{STop}_{k} \wedge Z_{+}$, where $T \mathrm{STop}_{k}$ is the Thom space. The inclusion of the 0 -section gives an embedding

$$
B \mathrm{STop}_{k} \rightarrow \mathrm{TSTop}_{k} \text {. }
$$

Given a map $f: S^{n+k} \rightarrow T \operatorname{STop}_{k} \wedge Z_{+}$, there is a homotopic map $f^{\prime}$ for which the composite

$$
S^{n+k} \stackrel{f^{\prime}}{\rightarrow} T \mathrm{STop}_{k} \wedge Z_{+} \stackrel{p_{1}}{\longrightarrow} T \mathrm{STop}_{k}
$$

(where $p_{1}$ is the projection) is transverse to the 0 -section. Then the oriented topological manifold $\left(p_{1} \circ f^{\prime}\right)^{-1}\left(B \mathrm{STop}_{k}\right)$ is equal to $\left(f^{\prime}\right)^{-1}\left(B \mathrm{STop}_{k} \times Z\right)$, and $i$ takes the homotopy class of $f$ to the bordism class of the composite

$$
\left(f^{\prime}\right)^{-1}\left(B \operatorname{STop}_{k} \times Z\right) \stackrel{f^{\prime}}{\rightarrow} B \operatorname{STop}_{k} \times Z \stackrel{p_{2}}{\rightarrow} Z .
$$

Next we observe that $i$ can be described using maps of spectra. Let $S_{\bullet}\left(T \operatorname{STop}_{k} \wedge Z_{+}\right)$be the singular complex, and let $S_{\bullet}^{\pitchfork}\left(T \operatorname{STop}_{k} \wedge Z_{+}\right)$be the sub-semisimplicial set consisting of maps $g: \Delta^{n} \rightarrow T \operatorname{STop}_{k} \wedge Z_{+}$for which the restriction of $p_{1} \circ g$ to each face is transverse to the 0 -section (cf. [LM13, Appendix B]). Let

$$
\left(M \mathrm{STop} \wedge Z_{+}\right)^{\pitchfork}
$$

be the spectrum whose $k$-th space is the geometric realization $\mid S_{\bullet}^{\pitchfork}\left(T \operatorname{STop}_{k} \wedge\right.$ $\left.Z_{+}\right) \mid$. Given a simplex $g$ of $S_{\bullet}^{\pitchfork}\left(\operatorname{TSTop}_{k} \wedge Z_{+}\right)$, we obtain an element of $\operatorname{ad}_{\text {STop }, Z}^{k}\left(\Delta^{n}\right)$ by taking each oriented simplex $(\sigma, o)$ to $\left(\left.g\right|_{\sigma}\right)^{-1}(B$ STop $\times Z)$; this gives a natural transformation

$$
I:\left(M \operatorname{STop} \wedge Z_{+}\right)^{\pitchfork} \rightarrow \mathbf{Q S T o p}, Z_{\text {. }}
$$

Next, transversality implies that the map

$$
\left|S_{\bullet}^{\pitchfork}\left(T \operatorname{STop}_{k} \wedge Z_{+}\right)\right| \rightarrow\left|S_{\bullet}\left(\operatorname{TSTop}_{k} \wedge Z_{+}\right)\right|
$$

is a weak equivalence (because by May67, Lemma 16.3, Definition 3.6, Example 1.5 and Lemma 1.5] each element of $\pi_{n}$ of the target is represented by an $n$-simplex whose faces are at the basepoint, and such a simplex can be deformed to one that is transverse, and similarly for homotopies), and so the map

$$
\left(M \operatorname{STop} \wedge Z_{+}\right)^{\pitchfork} \rightarrow M \text { STop } \wedge Z_{+}
$$

is a weak equivalence. The isomorphism $i$ is induced by the composite

$$
M \text { STop } \wedge Z_{+} \stackrel{\simeq}{\longleftarrow}\left(M \operatorname{STop} \wedge Z_{+}\right)^{\pitchfork} \stackrel{I}{\rightarrow} \mathbf{Q}_{\mathrm{STop}, Z}
$$

together with the isomorphism $\pi_{n} \mathbf{Q}_{\mathrm{STop}, Z} \cong\left(\Omega_{\mathrm{STop}}\right)_{n}(Z)$.

\footnotetext{
7 The referee has asked us to remind the reader that [LM13, Appendix B] uses the folk theorem that that there is an isomorphism $\Omega_{*}$ (STop) $\rightarrow \pi_{*}$ (MSTop) whose construction is similar to that on pages 19-20 of St68.
} 
Now consider the diagram

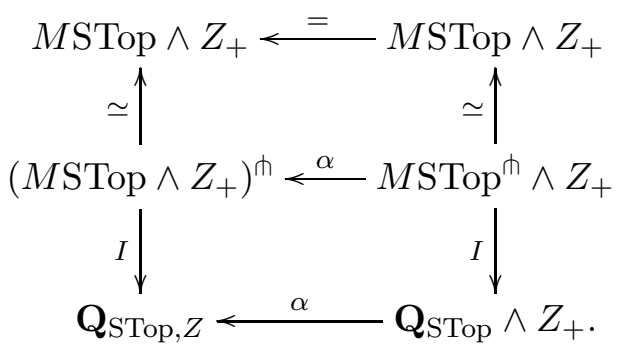

This homotopy commutes by naturality of the assembly map (since the assembly map for the functor $M S \operatorname{Sop} \wedge Z_{+}$is the identity map). On passage to homotopy groups, the right-hand vertical composite induces the map $j$ of diagram (9.5), and this shows that diagram (9.5) commutes as required.

In Par90, Pardon computes the IP bordism groups of a point to be

$$
\Omega_{n}^{\mathrm{IP}}(\mathrm{pt}) \cong \begin{cases}\mathbb{Z}, & n \equiv 0(4) \\ \mathbb{Z} / 2, & n \equiv 1(4), n>1 \\ 0, & \text { otherwise. }\end{cases}
$$

The isomorphisms are given by the signature (when $n \equiv 0(4)$ ) and the de Rham invariant (when $n \equiv 1(4)$ ). These groups are very close to

$$
L^{n}(\mathbb{Z}) \cong \begin{cases}\mathbb{Z}, & n \equiv 0(4) \\ \mathbb{Z} / 2, & n \equiv 1(4) \\ 0, & \text { otherwise. }\end{cases}
$$

Theorem 9.8. The map $\Omega_{n}^{\mathrm{IP}}(\mathrm{pt}) \rightarrow \mathrm{L}_{n}^{\bullet}(\mathrm{pt})=L^{n}(\mathbb{Z})$, given by (9.3) on a point, is an isomorphism for all $n \neq 1$.

Proof. If $n \equiv 2,3(4)$, then both $\Omega_{n}^{\mathrm{IP}}(\mathrm{pt})$ and $L^{n}(\mathbb{Z})$ vanish and the claim holds.

Suppose that $n \equiv 0(4)$. Then by (9.6), the signature is an isomorphism $\Omega_{n}^{\mathrm{IP}}(\mathrm{pt}) \cong \mathbb{Z}$. The signature of a symmetric Poincaré chain complex is an isomorphism $L^{n}(\mathbb{Z}) \cong \mathbb{Z}$. We shall show that our map $\Omega_{n}^{\mathrm{IP}}(\mathrm{pt}) \rightarrow L^{n}(\mathbb{Z})$ sends a generator to a generator. The complex projective space $\mathbb{C P}^{n / 2}$ represents a generator $\left[\mathbb{C P}^{n / 2}\right] \in \Omega_{n}^{\mathrm{IP}}(\mathrm{pt})$ since it is an IP-space and has signature 1 . Let $f$ be the map $f: \mathbb{C P}^{n / 2} \rightarrow$ pt. The diagram

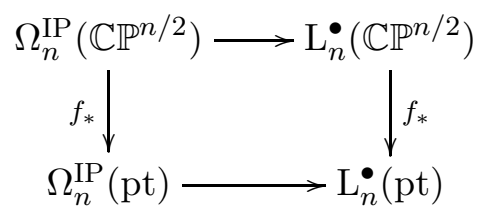

commutes, since $\Omega_{n}^{\mathrm{IP}}(-) \rightarrow \mathrm{L}_{n}^{\bullet}(-)$ is a natural transformation of homology theories (being induced by a spectrum level map). Since the assembly map 
$\alpha$ is a natural transformation of functors and $\mathbb{C P}^{n / 2}$ is simply connected, the diagram

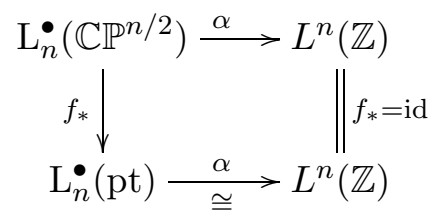

commutes as well. By construction, the top horizontal arrow of diagram (9.7) maps $\left[\mathrm{id}_{\mathbb{C P}^{n / 2}}\right] \in \Omega_{n}^{\mathrm{IP}}\left(\mathbb{C P}^{n / 2}\right)$ to the fundamental class $\left[\mathbb{C P}^{n / 2}\right]_{\mathbb{L}} \in$ $\mathrm{L}_{n}^{\bullet}\left(\mathbb{C P}^{n / 2}\right)$. By our Theorem 9.3 (iii), this class agrees with Ranicki's fundamental $\mathrm{L}^{\bullet}$-homology class for manifolds. The latter class is known to assemble to the Mischenko-Ranicki symmetric signature $\sigma^{*}\left(\mathbb{C P}^{n / 2}\right)$, which is a generator of $L^{n}(\mathbb{Z})$, since the ordinary signature of $\mathbb{C P}^{n / 2}$ is 1 . Now $f_{*}\left[\mathrm{id}_{\mathbb{C P}^{n / 2}}\right]=\left[\mathbb{C P}^{n / 2}\right] \in \Omega_{n}^{\mathrm{IP}}(\mathrm{pt})$ and thus by commutativity, the bottom arrows of the two diagrams must also map $\left[\mathbb{C P}^{n / 2}\right]$ to a generator and the claim is proved.

Suppose that $n \equiv 1(4)$ and $n>1$. Then according to (9.6), the de Rham invariant is an isomorphism $\Omega_{n}^{\mathrm{IP}}(\mathrm{pt}) \cong \mathbb{Z} / 2$. The de Rham invariant of a symmetric Poincaré chain complex is an isomorphism $L^{n}(\mathbb{Z}) \cong \mathbb{Z} / 2$. To show that our map $\Omega_{n}^{\mathrm{IP}}(\mathrm{pt}) \rightarrow L^{n}(\mathbb{Z})$ sends the generator to the generator, we can use the same argument as in the above case, replacing the signature by the de Rham invariant and replacing the complex projective spaces by simply connected smooth manifolds $M^{n}$ with nontrivial de Rham invariant. In dimension 5 , such a manifold is given by $M^{5}=\mathrm{SU}(3) / \mathrm{SO}(3)$. If $A$ is a manifold of dimension congruent $1 \bmod 4$ and $B$ a manifold of dimension congruent $0 \bmod 4$, then the de Rham invariant of the product $A \times B$ is the de Rham invariant of $A$ multiplied by the signature of $B$. Thus, the manifolds $M^{n}=M^{5} \times \mathbb{C P}^{(n-5) / 2}$, all have de Rham invariant 1 and are simply connected. The Mischenko-Ranicki symmetric signature $\sigma^{*}\left(M^{n}\right)$ is then the generator of $L^{n}(\mathbb{Z})$, since $M^{n}$ has nontrivial de Rham invariant.

Remark 9.9. In degree $n=1$, there is a discrepancy: $\Omega_{1}^{\mathrm{IP}}(\mathrm{pt})=0$, while $L^{1}(\mathbb{Z}) \cong \mathbb{Z} / 2$.

\section{The ASSEMbly of the FUndamental Class}

The goal of this section is to prove that $[X]_{\mathbb{L}}$ assembles to the symmetric signature $\sigma_{\mathrm{IP}}^{*}(X)$.

Before giving the precise statement (Theorem 10.12) we need some preliminary work. In order to construct the relevant assembly map, we first need a functorial model for ad ${ }^{\mathbb{Z}\left[\pi_{1} Z\right]} ; \operatorname{ad}^{\mathbb{Z}\left[\pi_{1} Z\right]}$ is not a functor of $Z$ because the category of modules over $\mathbb{Z}\left[\pi_{1} Z\right]$ is not a functor of $Z$ (see [LM13, Section 13] 
for an explanation of this issue in the context of $R$ modules) 8 Accordingly, in Subsection 10.1 we give a functorial model for the category of modules over $\mathbb{Z}\left[\pi_{1} Z\right]$, and in Subsection 10.2 we use this to construct an ad theory $\operatorname{ad}_{\mathbb{F}}^{\mathbb{Z}\left[\pi_{1} Z\right]}$ which is a functor of $Z$ and has a canonical map to $\operatorname{ad}^{\mathbb{Z}\left[\pi_{1} Z\right]}$ which is an isomorphism on bordism groups. Subsection 10.3 gives the statement of the main theorem and an outline of the proof; the details of the proof are given in Subsections 10.4 10.13.

Convention 10.1. Throughout this section, $Z$ will denote a path-connected space.

10.1. A functorial model for the category of modules over $\mathbb{Z}\left[\pi_{1} Z\right]$. We use the terminology and notation of [LM, Subsection B.5] (which the reader should consult before continuing) $\sqrt{9}$ together with:

Notation 10.2. When possible we denote a schematic $R$ module by a single letter $\mathbb{A}$ rather than by a triple $(\mathbb{M}, \mathbb{N}, T)$. (Then $R\langle\mathbb{A}\rangle$ denotes the quotient of the free $R$ module $R\langle\mathbb{M}\rangle$ by the image of the map $T: R\langle\mathbb{N}\rangle \rightarrow R\langle\mathbb{M}\rangle$.)

To lighten the notation, we will denote $\mathbb{Z}\left[\pi_{1} Z\right]$ by $\mathcal{R}(Z)$ and $\mathbb{Z}\left[\pi_{1}(Z, z)\right]$ by $\mathcal{R}(Z)_{z}$ throughout this subsection.

Let us define a schematic $\mathcal{R}(Z)$ module to be a pair $(z, \mathbb{A})$ where $z$ is an element of $Z$ and $\mathbb{A}$ is a schematic $\mathcal{R}(Z)_{z}$ module. If we think of $\mathcal{R}(Z)_{z}$ as a subcategory of $\mathcal{R}(Z)$ and of $\mathcal{R}(Z)_{z}\langle\mathbb{A}\rangle$ as a module over this subcategory, then the Kan extension 10 of $\mathcal{R}(Z)_{z}\langle\mathbb{A}\rangle$ is an $\mathcal{R}(Z)$ module which will be denoted $\mathcal{R}(Z)\langle z, \mathbb{A}\rangle$.

Here is an explicit description of $\mathcal{R}(Z)\langle z, \mathbb{A}\rangle$. If we denote the set of path homotopy classes of paths from $z$ to $z^{\prime}$ by $\mathfrak{p}_{z, z^{\prime}}$, then $\mathcal{R}(Z)\langle z, \mathbb{A}\rangle$ is defined on objects by letting $\mathcal{R}(Z)\langle z, \mathbb{A}\rangle_{z^{\prime}}$ be the quotient of

$$
\operatorname{Mor}_{\mathcal{R}(Z)}\left(z, z^{\prime}\right) \otimes \mathcal{R}(Z)_{z}\langle\mathbb{A}\rangle \cong \bigoplus_{\delta \in \mathfrak{p}_{z, z^{\prime}}} \mathcal{R}(Z)_{z}\langle\mathbb{A}\rangle
$$

by the following equivalence relation: if $(\delta, a)$ denotes the copy of an element $a \in \mathcal{R}(Z)_{z}\langle\mathbb{A}\rangle$ in the $\delta$ summand and $\gamma$ is the class of a loop at $z$ then

\footnotetext{
${ }^{8}$ Briefly, the point is that the obvious way of trying to make the category of $R$ modules a functor of $R$ doesn't give a functor because $R_{3} \otimes_{R_{2}}\left(R_{2} \otimes_{R_{1}} M\right)$ is canonically isomorphic to, but not the same module as, $R_{3} \otimes_{R_{1}} M$.

${ }^{9}$ For the benefit of the reader who doesn't want to consult [LM, Subsection B.5], here are the key definitions:

We define the category of schematic free $R$ modules as follows. An object is a set $\mathbb{M}$. This should be thought of as representing the free $R$ module generated by $\mathbb{M}$, which we denote by $R\langle\mathbb{M}\rangle$. We define a map $\mathbb{M} \rightarrow \mathbb{M}^{\prime}$ to be a map of $R$-modules $R\langle\mathbb{M}\rangle \rightarrow R\left\langle\mathbb{M}^{\prime}\right\rangle$.

We define the category of schematic $R$ modules as follows. An object of this category is a triple $(\mathbb{M}, \mathbb{N}, T)$, where $\mathbb{M}$ and $\mathbb{N}$ are schematic free $R$ modules and $T$ is a map $\mathbb{N} \rightarrow \mathbb{M}$. Such a triple should be thought of as representing the quotient of $R\langle\mathbb{M}\rangle$ by the image of $T$; we write $R\langle(\mathbb{M}, \mathbb{N}, T)\rangle$ for this quotient.

${ }^{10}$ See Appendix B.
} 
$(\delta \gamma, a) \sim(\delta, \gamma a) ; 11$ it follows that for any $\delta \in \mathfrak{p}_{z, z^{\prime}}$ the map which takes $a$ to $(\delta, a)$ is an isomorphism $\mathcal{R}(Z)_{z}\langle\mathbb{A}\rangle \rightarrow \mathcal{R}(Z)\langle z, \mathbb{A}\rangle_{z^{\prime}}$. The action of the morphisms is given as follows: if $\epsilon \in \mathfrak{p}_{z^{\prime}, z^{\prime \prime}}$ is thought of as a morphism from $z^{\prime}$ to $z^{\prime \prime}$ then $\epsilon$ takes $(\delta, a)$ to $(\epsilon \delta, a)$.

We define a map from $(z, \mathbb{A})$ to $\left(z^{\prime}, \mathbb{A}^{\prime}\right)$ to be a homomorphism of $\mathcal{R}(Z)$ modules $\mathcal{R}(Z)\langle z, \mathbb{A}\rangle \rightarrow \mathcal{R}(Z)\left\langle z^{\prime}, \mathbb{A}^{\prime}\right\rangle$.

Lemma 10.3. The functor from schematic $\mathcal{R}(Z)$ modules to $\mathcal{R}(Z)$ modules which takes $(z, \mathbb{A})$ to $\mathcal{R}(Z)\langle z, \mathbb{A}\rangle$ is an equivalence of categories.

Proof. The functor is the identity on morphism sets, so it's only necessary to show that every $\mathcal{R}(Z)$ module $\mathcal{M}$ is isomorphic to one of the form $\mathcal{R}(Z)\langle z, \mathbb{A}\rangle$. Fix $z \in Z$. By [LM, Lemma B.19], there is an $\mathbb{A}$ with an isomorphism $\mathcal{R}(Z)_{z}\langle\mathbb{A}\rangle \rightarrow \mathcal{M}_{z}$, and the explicit description of $\mathcal{R}(Z)\langle z, \mathbb{A}\rangle$ given above shows that this isomorphism induces an isomorphism $\mathcal{R}(Z)\langle z, \mathbb{A}\rangle \rightarrow \mathcal{M}$.

A schematic chain complex $\mathbb{C}$ over $\mathbb{Z}\left[\pi_{1} Z\right]$ is a sequence of schematic $\mathbb{Z}\left[\pi_{1} Z\right]$ modules and maps. We write $\mathbb{Z}\left[\pi_{1} Z\right]\langle\mathbb{C}\rangle$ for the corresponding sequence of $\mathbb{Z}\left[\pi_{1} Z\right]$ modules and maps. A map $\mathbb{C} \rightarrow \mathbb{C}^{\prime}$ of schematic chain complexes is a map of $\mathbb{Z}\left[\pi_{1} Z\right]$ chain complexes $\mathbb{Z}\left[\pi_{1} Z\right]\langle\mathbb{C}\rangle \rightarrow \mathbb{Z}\left[\pi_{1} Z\right]\left\langle\mathbb{C}^{\prime}\right\rangle$.

Now let $g: Z_{1} \rightarrow Z_{2}$ be a continuous function.

Notation 10.4. For each $z \in Z$ let

$$
g_{z}: \mathcal{R}\left(Z_{1}\right)_{z} \rightarrow \mathcal{R}\left(Z_{2}\right)_{g(z)}
$$

be the induced homomorphism. Let

$$
g_{*}: \mathcal{R}\left(Z_{1}\right) \rightarrow \mathcal{R}\left(Z_{2}\right)
$$

be the induced functor.

We want to show that $g$ induces a functor $g_{\text {sch }}$ from the category of schematic $\mathcal{R}\left(Z_{1}\right)$ modules to the category of schematic $\mathcal{R}\left(Z_{2}\right)$ modules. First we define $g_{\text {sch }}$ on objects: for a schematic $\mathcal{R}\left(Z_{1}\right)$ module $(z, \mathbb{A})$ we define $g_{\text {sch }}(z, \mathbb{A})$ to be the schematic $\mathcal{R}\left(Z_{2}\right)$ module $\left(g(z),\left(g_{z}\right)_{\text {sch }} \mathbb{A}\right)$. To define $g_{\text {sch }}$ on maps we need a lemma.

Lemma 10.5. There is a canonical isomorphism

$$
\mathcal{R}\left(Z_{2}\right)\left\langle g_{\mathrm{sch}}(z, \mathbb{A})\right\rangle \cong \operatorname{Kan}_{g_{*}} \mathcal{R}\left(Z_{1}\right)\langle z, \mathbb{A}\rangle
$$

Proof. Let $i: \mathcal{R}\left(Z_{1}\right)_{z} \rightarrow \mathcal{R}\left(Z_{1}\right)$ and $j: \mathcal{R}\left(Z_{2}\right)_{g(z)} \rightarrow \mathcal{R}\left(Z_{2}\right)$ be the inclusions. We have

$$
\begin{array}{r}
\left.\mathcal{R}\left(Z_{2}\right)\left\langle g_{\mathrm{sch}}(z, \mathbb{A})\right\rangle=\mathcal{R}\left(Z_{2}\right)\left\langle g(z),\left(g_{z}\right)_{\operatorname{sch}} \mathbb{A}\right\rangle=\operatorname{Kan}_{j}\left(\mathcal{R}\left(Z_{2}\right)_{g(z)}\left\langle\left(g_{z}\right)_{\operatorname{sch}} \mathbb{A}\right)\right\rangle\right) \\
\cong \operatorname{Kan}_{j}\left(\operatorname{Kan}_{g_{z}}\left(\mathcal{R}\left(Z_{1}\right)_{z}\langle\mathbb{A}\rangle\right)\right) \cong \operatorname{Kan}_{j \circ g_{z}}\left(\mathcal{R}\left(Z_{1}\right)_{z}\langle\mathbb{A}\rangle\right) \\
=\operatorname{Kan}_{g_{*} \circ i}\left(\mathcal{R}\left(Z_{1}\right)_{z}\langle\mathbb{A}\rangle\right) \cong \operatorname{Kan}_{g_{*}}\left(\operatorname{Kan}_{i}\left(\mathcal{R}\left(Z_{1}\right)_{z}\langle\mathbb{A}\rangle\right)\right) \\
=\operatorname{Kan}_{g_{*}} \mathcal{R}\left(Z_{1}\right)\langle z, \mathbb{A}\rangle
\end{array}
$$

\footnotetext{
${ }^{11}$ Note that we denote composition of path homotopy classes by letting $\delta \gamma$ be "first $\gamma$, then $\delta$ ", analogously to composition of functions.
} 
where the equalities in the first line are definitions, the first isomorphism in the second line is [LM, Equation (B.2) in Subsection B.5] and Example B.2 and the second is Equation (B.1), the first equality in the third line is obvious and the isomorphism is Equation (B.1), and the last equality follows from the definition of $\mathcal{R}\left(Z_{1}\right)\langle z, \mathbb{A}\rangle$.

Now for a map

$$
t:(z, \mathbb{A}) \rightarrow\left(z^{\prime}, \mathbb{A}^{\prime}\right)
$$

we define

$$
g_{\mathrm{sch}} t: g_{\mathrm{sch}}(z, \mathbb{A}) \rightarrow g_{\mathrm{sch}}\left(z^{\prime}, \mathbb{A}^{\prime}\right)
$$

to be the map determined by the diagram

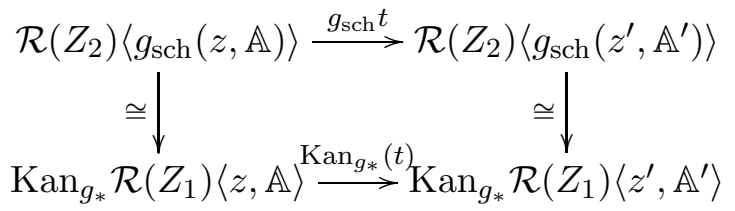

where the bottom arrow is induced by $t$.

With these definitions, $g_{\text {sch }}$ is a functor from schematic $\mathcal{R}\left(Z_{1}\right)$ modules to schematic $\mathcal{R}\left(Z_{2}\right)$ modules. If $h$ is a continuous function $Z_{2} \rightarrow Z_{3}$ we have $(h \circ g)_{\text {sch }}=h_{\text {sch }} \circ g_{\text {sch }}$, so the category of schematic $\mathcal{R}(Z)$ modules is a functor of $Z$.

10.2. A functorial model for $\operatorname{ad}^{\mathbb{Z}\left[\pi_{1} Z\right]}$.

Notation 10.6. As usual, let $W$ be the standard resolution of $\mathbb{Z}$ by $\mathbb{Z}[\mathbb{Z} / 2]$ modules.

Definition 10.7. (i) A schematic quasi-symmetric complex of dimension $n$ over $\mathbb{Z}\left[\pi_{1} Z\right]$ is a pair $(\mathbb{C}, \varphi)$, where $\mathbb{C}$ is a schematic chain complex over $\mathbb{Z}\left[\pi_{1} Z\right]$ for which $\mathbb{Z}\left[\pi_{1} Z\right]\langle\mathbb{C}\rangle$ is homotopy finite and $\varphi$ is a $\mathbb{Z} / 2$-equivariant map

$$
W \rightarrow\left(\mathbb{Z}\left[\pi_{1} Z\right]\langle\mathbb{C}\rangle\right)^{t} \otimes_{\mathbb{Z}\left[\pi_{1} Z\right]} \mathbb{Z}\left[\pi_{1} Z\right]\langle\mathbb{C}\rangle
$$

of graded abelian groups which raises degrees by $n$.

(ii) We define a category $\mathcal{A}_{\mathrm{sch}}^{\mathbb{Z}\left[\pi_{1} Z\right]}$ as follows. The objects are the schematic quasi-symmetric complexes over $\mathbb{Z}\left[\pi_{1} Z\right]$. A morphism $(\mathbb{C}, \varphi) \rightarrow\left(\mathbb{C}^{\prime}, \varphi^{\prime}\right)$ is a map of $\mathbb{Z}\left[\pi_{1} Z\right]$ chain complexes $f: \mathbb{Z}\left[\pi_{1} Z\right]\langle\mathbb{C}\rangle \rightarrow \mathbb{Z}\left[\pi_{1} Z\right]\left\langle\mathbb{C}^{\prime}\right\rangle$ such that if $\operatorname{dim} \varphi=\operatorname{dim} \varphi^{\prime}$ then $\left(f^{t} \otimes f\right) \circ \varphi=\varphi^{\prime}$.

There is a morphism

$$
\Theta: \mathcal{A}_{\mathrm{sch}}^{\mathbb{Z}\left[\pi_{1} Z\right]} \rightarrow \mathcal{A}^{\mathbb{Z}\left[\pi_{1} Z\right]}
$$

of $\mathbb{Z}$-graded categories which takes $(\mathbb{C}, \varphi)$ to $\left(\mathbb{Z}\left[\pi_{1} Z\right]\langle\mathbb{C}\rangle, \varphi\right)$; this is an equivalence of categories.

Definition 10.8. A $K$-ad with values in $\mathcal{A}_{\text {sch }}^{\mathbb{Z}\left[\pi_{1} Z\right]}$ is a pre $K$ ad $F$ for which $\Theta \circ F$ is a $K$-ad. 
We write $\operatorname{ad}_{\text {sch }}^{\mathbb{Z}\left[\pi_{1} Z\right]}(K)$ for the set of $K$-ads with values in $\mathcal{A}_{\text {sch }}^{\mathbb{Z}\left[\pi_{1} Z\right]}$.

Proposition 10.9. (i) $\operatorname{ad}_{\mathrm{sch}}^{\mathbb{Z}\left[\pi_{1} Z\right]}$ is an ad theory.

(ii) $\Theta$ induces a morphism of ad theories which is an isomorphism of bordism groups.

This is an easy consequence of the fact that $\operatorname{ad}^{\mathbb{Z}\left[\pi_{1} Z\right]}$ is an ad theory and LM, Lemma B.23].

Next we consider functoriality. Now let $g: Z \rightarrow Z^{\prime}$ be a continuous function. Define a functor

$$
g_{\mathrm{sch}}^{\mathrm{sym}}: \mathcal{A}_{\mathrm{sch}}^{\mathbb{Z}\left[\pi_{1} Z\right]} \rightarrow \mathcal{A}_{\mathrm{sch}}^{\mathbb{Z}\left[\pi_{1} Z^{\prime}\right]}
$$

by

$$
g_{\mathrm{sch}}^{\mathrm{sym}}(\mathbb{C}, \varphi)=\left(g_{\mathrm{sch}}(\mathbb{C}), \psi\right),
$$

where $\psi$ is the composite

$$
\begin{aligned}
W \stackrel{\varphi}{\rightarrow}\left(\mathbb{Z}\left[\pi_{1} Z\right]\langle\mathbb{C}\rangle\right)^{t} \otimes_{\mathbb{Z}\left[\pi_{1} Z\right]} \mathbb{Z}\left[\pi_{1} Z\right]\langle\mathbb{C}\rangle \stackrel{\iota \otimes \iota}{\longrightarrow} \\
\left(\operatorname{Kan}_{g_{*}}\left(\mathbb{Z}\left[\pi_{1} Z\right]\langle\mathbb{C}\rangle\right)\right)^{t} \otimes_{\mathbb{Z}\left[\pi_{1} Z^{\prime}\right]} \operatorname{Kan}_{g_{*}}\left(\mathbb{Z}\left[\pi_{1} Z\right]\langle\mathbb{C}\rangle\right) \cong \\
\left(\mathbb{Z}\left[\pi_{1} Z^{\prime}\right]\left\langle g_{\text {sch }}(\mathbb{C})\right\rangle\right)^{t} \otimes_{\mathbb{Z}\left[\pi_{1} Z^{\prime}\right]} \mathbb{Z}\left[\pi_{1} Z^{\prime}\right]\left\langle g_{\text {sch }}(\mathbb{C})\right\rangle,
\end{aligned}
$$

where $\iota$ is given in Appendix $\mathrm{B}$ and the isomorphism is given by Lemma 10.5 .

Proposition 10.10. Let $g: Z \rightarrow Z^{\prime}$ and $g^{\prime}: Z^{\prime} \rightarrow Z^{\prime \prime}$ be continuous functions. Then

$$
\left(g^{\prime} g\right)_{\mathrm{sch}}^{\mathrm{sym}}=\left(g^{\prime}\right)_{\mathrm{sch}}^{\mathrm{sym}} g_{\mathrm{sch}}^{\mathrm{sym}} .
$$

Proof. First we show that $\left(g^{\prime} g\right)_{\mathrm{sch}}^{\mathrm{sym}}=\left(g^{\prime}\right)_{\mathrm{sch}}^{\mathrm{sym}} g_{\mathrm{sch}}^{\mathrm{sym}}$ on objects. Let $(\mathbb{C}, \varphi)$

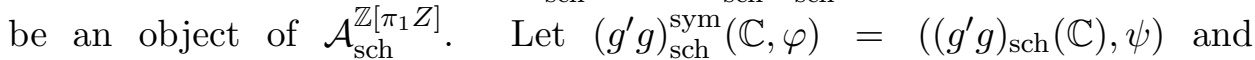
let $\left(g^{\prime}\right)_{\mathrm{sch}}^{\mathrm{sym}} g_{\mathrm{sch}}^{\mathrm{sym}}(\mathbb{C}, \varphi)=\left(g_{\mathrm{sch}}^{\prime} g_{\mathrm{sch}}(\mathbb{C}), \psi^{\prime}\right)$. We have already seen that $\left(g^{\prime} g\right)_{\mathrm{sch}}(\mathbb{C})=g_{\mathrm{sch}}^{\prime} g_{\mathrm{sch}}(\mathbb{C})$. For the proof that $\psi=\psi^{\prime}$, first let $(z, \mathbb{A})$ be a schematic $\mathbb{Z}\left[\pi_{1} Z\right]$ module and consider the diagram

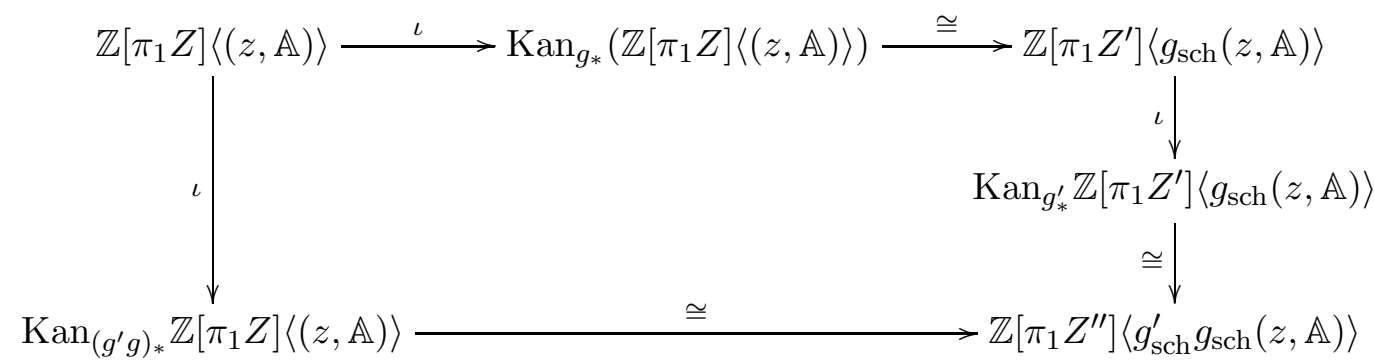

By definition, the upper left corner is a Kan extension, and by the universal property of the Kan extension (in Appendix (B) it suffices to show that the clockwise and counterclockwise composites in the diagram agree when restricted to $\mathbb{Z}\left[\pi_{1}(Z, z)\right]\langle\mathbb{A}\rangle$, and this is a routine verification. The proof that $\psi=\psi^{\prime}$ is a straightforward consequence of commutativity of the diagram. 
Next we show that $\left(g^{\prime} g\right)_{\text {sch }}^{\mathrm{sym}}=\left(g^{\prime}\right)_{\text {sch }}^{\mathrm{sym}} g_{\text {sch }}^{\mathrm{sym}}$ on maps. Let $f:(\mathbb{C}, \varphi) \rightarrow$ $\left(\mathbb{C}^{\prime}, \varphi^{\prime}\right)$ be a map, which simply means that $f$ is a map $\mathbb{Z}\left[\pi_{1} Z\right]\langle\mathbb{C}\rangle \rightarrow$ $\mathbb{Z}\left[\pi_{1} Z\right]\left\langle\mathbb{C}^{\prime}\right\rangle$ (with $\left(f^{t} \otimes f\right) \circ \varphi=\varphi^{\prime}$ if $\left.\operatorname{dim} \varphi=\operatorname{dim} \varphi^{\prime}\right)$. First we observe that, for any schematic $\mathbb{Z}\left[\pi_{1} Z\right]$ module $(z, \mathbb{A})$, the diagram

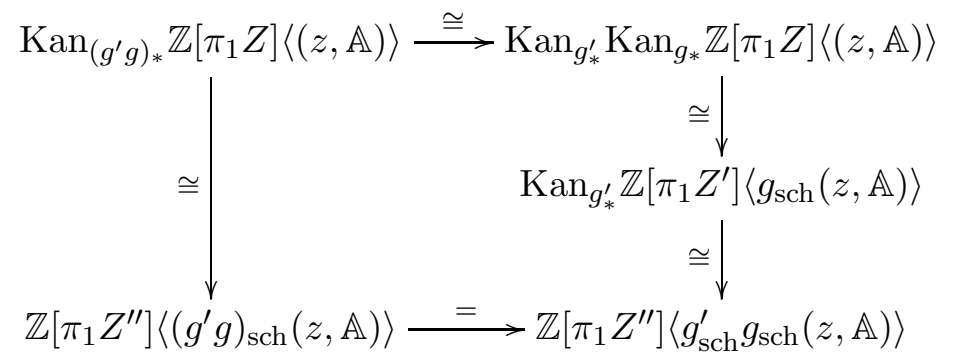

commutes, as the reader can check; the fact that $\left(g^{\prime} g\right)_{\mathrm{sch}}^{\mathrm{sym}}(f)=$ $\left(g^{\prime}\right)_{\text {sch }}^{\mathrm{sym}} g_{\mathrm{sch}}^{\mathrm{sym}}(f)$ is an easy consequence of this and the definitions.

Proposition 10.11. $g_{\text {sch }}^{\text {sym }}$ takes ads to ads.

Proof. Let $F \in \operatorname{ad}_{\mathrm{sch}}^{\mathbb{Z}\left[\pi_{1} Z\right]}(K)$, and write

$$
F(\sigma, o)=\left(\mathbb{C}_{\sigma}, \varphi_{\sigma, o}\right)
$$

Next let $z \in Z$ and observe that $\Theta \circ F$ determines a pre $K$-ad $(\Theta \circ F)_{z}$ with

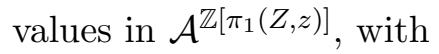

$$
(\Theta \circ F)_{z}(\sigma, o)=\left(\mathbb{Z}\left[\pi_{1} Z\right]\left\langle\mathbb{C}_{\sigma}\right\rangle_{z},\left(\varphi_{\sigma, o}\right)_{z}\right)
$$

where $\left(\varphi_{\sigma, o}\right)_{z}$ is the composite

$$
\begin{aligned}
W \stackrel{\varphi_{\sigma, o}}{\longrightarrow}\left(\mathbb{Z}\left[\pi_{1} Z\right]\left\langle\mathbb{C}_{\sigma}\right\rangle\right)^{t} \otimes_{\mathbb{Z}\left[\pi_{1} Z\right]} \mathbb{Z}\left[\pi_{1} Z\right]\left\langle\mathbb{C}_{\sigma}\right\rangle \\
\left.\left.\cong\left(\mathbb{Z}\left[\pi_{1} Z\right]\left\langle\mathbb{C}_{\sigma}\right\rangle\right)_{z}\right)^{t} \otimes_{\mathbb{Z}\left[\pi_{1}(Z, z)\right]} \mathbb{Z}\left[\pi_{1} Z\right]\left\langle\mathbb{C}_{\sigma}\right\rangle\right)_{z} .
\end{aligned}
$$

Using Definition 10.8 and Remarks 7.1 and 7.2 , we see that $(\Theta \circ F)_{z}$ is an ad.

Now let $G=\Theta \circ g_{\mathrm{sch}}^{\mathrm{sym}} \circ F$ and write

$$
G(\sigma, o)=\left(\mathbb{Z}\left[\pi_{1} Z^{\prime}\right]\left\langle g_{\mathrm{sch}}\left(\mathbb{C}_{\sigma}\right)\right\rangle, \psi_{\sigma, o}\right)
$$

we need to show that $G \in \operatorname{ad}^{\mathbb{Z}\left[\pi_{1} Z^{\prime}\right]}(K)$. First we observe that, by Lemma 10.5 and the definition of $g_{\mathrm{sch}}^{\mathrm{sym}}, G$ is isomorphic to the pre $K$-ad $G^{\prime}$ with

$$
G^{\prime}(\sigma, o)=\left(\operatorname{Kan}_{g_{*}}\left(\mathbb{Z}\left[\pi_{1} Z\right]\left\langle\mathbb{C}_{\sigma}\right\rangle, \psi_{\sigma, o}^{\prime}\right),\right.
$$

where $\psi_{\sigma, o}^{\prime}$ is the composite

$$
\begin{aligned}
W \stackrel{\varphi_{\sigma, o}}{\longrightarrow}\left(\mathbb{Z}\left[\pi_{1} Z\right]\left\langle\mathbb{C}_{\sigma}\right\rangle\right)^{t} \otimes_{\mathbb{Z}\left[\pi_{1} Z\right]} \mathbb{Z}\left[\pi_{1} Z\right]\left\langle\mathbb{C}_{\sigma}\right\rangle \stackrel{\iota \otimes \iota}{\longrightarrow} \\
\quad\left(\operatorname{Kan}_{g_{*}}\left(\mathbb{Z}\left[\pi_{1} Z\right]\left\langle\mathbb{C}_{\sigma}\right\rangle\right)\right)^{t} \otimes_{\mathbb{Z}\left[\pi_{1} Z^{\prime}\right]} \operatorname{Kan}_{g_{*}}\left(\mathbb{Z}\left[\pi_{1} Z\right]\left\langle\mathbb{C}_{\sigma}\right\rangle\right),
\end{aligned}
$$

so it suffices to show $G^{\prime}$ is an ad. Now (recalling that we chose $z \in Z$ ), $G^{\prime}$ determines a pre $K$-ad $G_{g(z)}^{\prime}$ with

$$
G_{g(z)}^{\prime}(\sigma, o)=\left(\operatorname{Kan}_{g_{*}}\left(\mathbb{Z}\left[\pi_{1} Z\right]\left\langle\mathbb{C}_{\sigma}\right\rangle\right)_{g(z)},\left(\psi_{\sigma, o}^{\prime}\right)_{g(z)}\right)
$$


where $\left(\psi_{\sigma, o}^{\prime}\right)_{g(z)}$ is the composite

$$
\begin{aligned}
W \stackrel{\psi_{\sigma, o}^{\prime}}{\longrightarrow} & \left(\operatorname{Kan}_{g_{*}}\left(\mathbb{Z}\left[\pi_{1} Z\right]\left\langle\mathbb{C}_{\sigma}\right\rangle\right)\right)^{t} \otimes_{\mathbb{Z}\left[\pi_{1} Z^{\prime}\right]} \operatorname{Kan}_{g_{*}}\left(\mathbb{Z}\left[\pi_{1} Z\right]\left\langle\mathbb{C}_{\sigma}\right\rangle\right) \\
& \cong\left(\operatorname{Kan}_{g_{*}}\left(\mathbb{Z}\left[\pi_{1} Z\right]\left\langle\mathbb{C}_{\sigma}\right\rangle\right)\right)_{g(z)}^{t} \otimes_{\mathbb{Z}\left[\pi_{1}\left(Z^{\prime}, g(z)\right)\right]} \operatorname{Kan}_{g_{*}}\left(\mathbb{Z}\left[\pi_{1} Z\right]\left\langle\mathbb{C}_{\sigma}\right\rangle\right)_{g(z)}
\end{aligned}
$$

and it suffices (using Remarks 7.1 and 7.2 ) to show that $G_{g(z)}^{\prime}$ is an ad.

Finally, we observe that, by Remark B.3, $G_{g(z)}^{\prime}$ is isomorphic to the pre $K$-ad $H$ with

$$
H(\sigma, o)=\left(\mathbb{Z}\left[\pi_{1}\left(Z^{\prime}, g(z)\right] \otimes_{\mathbb{Z}\left[\pi_{1}(Z, z)\right]}\left(\mathbb{Z}\left[\pi_{1} Z\right]\left\langle\mathbb{C}_{\sigma}\right\rangle\right)_{z}, \chi_{\sigma, o}\right)\right.
$$

where $\chi_{\sigma, o}$ is the composite

$$
\begin{aligned}
& W \stackrel{\left(\varphi_{\sigma, o}\right)_{z}}{\longrightarrow}(\mathbb{Z}[\left.\left.\left.\left.\pi_{1} Z\right]\left\langle\mathbb{C}_{\sigma}\right\rangle\right)_{z}\right)^{t} \otimes_{\mathbb{Z}\left[\pi_{1}(Z, z)\right]} \mathbb{Z}\left[\pi_{1} Z\right]\left\langle\mathbb{C}_{\sigma}\right\rangle\right)_{z} \\
& \rightarrow\left(\mathbb{Z}\left[\pi_{1}\left(Z^{\prime}, g(z)\right] \otimes_{\mathbb{Z}\left[\pi_{1}(Z, z)\right]}\left(\mathbb{Z}\left[\pi_{1} Z\right]\left\langle\mathbb{C}_{\sigma}\right\rangle\right)_{z}\right)^{t}\right. \\
& \otimes_{\mathbb{Z}\left[\pi_{1}\left(Z^{\prime}, g(z)\right]\right.} \mathbb{Z}\left[\pi_{1}\left(Z^{\prime}, g(z)\right] \otimes_{\mathbb{Z}\left[\pi_{1}(Z, z)\right]}\left(\mathbb{Z}\left[\pi_{1} Z\right]\left\langle\mathbb{C}_{\sigma}\right\rangle\right)_{z},\right.
\end{aligned}
$$

and $H$ is an ad by $\left[\mathrm{LM}\right.$, Lemma B.15], using the fact that $(\Theta \circ F)_{z}$ is an ad.

Combining Propositions 10.10 and 10.11 we see that that $\operatorname{ad}_{\mathrm{sch}}^{\mathbb{Z}\left[\pi_{1} Z\right]}$ is a functor of $Z$.

10.3. Statement of the main theorem of this section, and outline of the proof. Since $\operatorname{ad}_{\mathrm{sch}, \geq 0}^{\mathbb{Z}\left[\pi_{1} Z\right]}$ is a homotopy invariant functor of $Z$, there is an assembly map

$$
\alpha: Z_{+} \wedge \mathbf{Q}_{\mathrm{sch}, \geq 0}^{\mathbb{Z}} \rightarrow \mathbf{Q}_{\mathrm{sch}, \geq 0}^{\mathbb{Z}\left[\pi_{1} Z\right]}
$$

Our main theorem in this section is

Theorem 10.12. The image of $[X]_{\mathbb{L}}$ under the composite

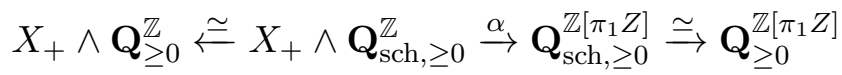

is the symmetric signature $\sigma_{\mathrm{IP}}^{*}(X)$ given by Definition 8.4 .

For the proof, we will use the following functors and natural transformations:

$$
\Phi \stackrel{\simeq}{\longleftarrow} \Phi^{\prime \operatorname{sig}} \rightarrow \Psi^{\prime \prime} \cong \Psi^{\prime} \rightarrow \Psi
$$

Here

- $\Phi$ is the functor

$$
\Phi(Z)=\mathbf{Q}_{\mathrm{IP}, Z}
$$

defined in Section 9,

- $\Phi^{\prime}$, which will be defined in Subsection 10.10, is a version of $\Phi$ which incorporates a choice of fundamental class (cf. the ad theory ad IPFun defined in Subsection 6.1),

- $\Psi$ is the functor

$$
\Psi(Z)=\mathbf{Q}_{\mathrm{sch}, \geq 0}^{\mathbb{Z}\left[\pi_{1} Z\right]}
$$


- $\Psi^{\prime}$ will be defined in Subsection 10.7, it is a functorial version of the ad theory $\operatorname{ad}_{\mathrm{fin}}^{\mathbb{Z}\left[\pi_{1} Z\right]}$ defined in Remark 7.6. It is needed because there is no reasonable map from $\Psi$ to $\Psi^{\prime \prime}$ or from $\Psi^{\prime \prime}$ to $\Psi$,

- the natural transformation $\Psi^{\prime} \rightarrow \Psi$ will be defined in Subsection 10.7, and the natural transformation $\Psi^{\prime} \rightarrow \Psi^{\prime \prime}$ will be defined in Subsection 10.8 .

- $\Psi^{\prime \prime}$, which will be defined in Subsection 10.5, is a functorial version of $\mathbf{Q}_{\mathrm{rel}, \geq 0}^{\mathbb{Z}\left[\pi_{1} Z\right]}$ (which was defined in Section 8 ),

- the natural transformation sig, which is a version of the symmectric signature, will be defined in Subsection 10.11.

Now consider the diagram

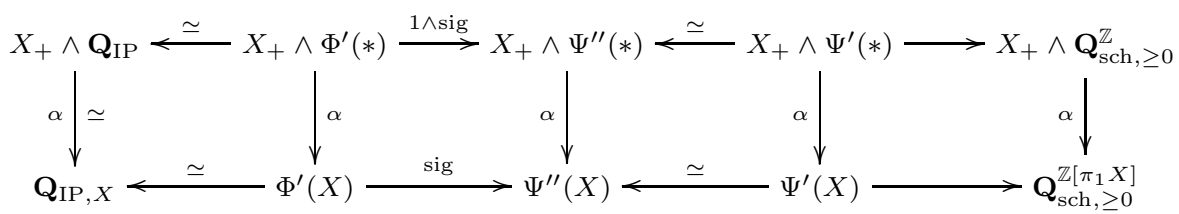

This diagram commutes up to homotopy by the naturality of the assembly map ([WW95, Theorem 1.1]). Now Theorem 10.12 is immediate from the following two lemmas, which will be proved in Subsections 10.12 and 10.13 respectively.

Lemma 10.13. The image of $\alpha^{-1}[X]_{\mathrm{IP}}$ along the top row of the diagram above followed by the map $X_{+} \wedge \mathbf{Q}_{\mathrm{sch}, \geq 0}^{\mathbb{Z}} \rightarrow X_{+} \wedge \mathbf{Q}_{\geq 0}^{\mathbb{Z}}$ is $[X]_{\mathbb{L}}$.

Lemma 10.14. The image of $[X]_{\mathrm{IP}}$ along the bottom row followed by the $\operatorname{map} \mathbf{Q}_{\mathrm{sch}, \geq 0}^{\mathbb{Z}\left[\pi_{1} X\right]} \rightarrow \mathbf{Q}_{\geq 0}^{\mathbb{Z}\left[\pi_{1} X\right]}$ is $\sigma_{\mathrm{IP}}^{*}(X)$.

10.4. Background for the functor $\Psi^{\prime \prime}$. In this subsection and the next our goal is to give a functorial version of $\operatorname{ad}_{\mathrm{rel}}^{\mathbb{Z}\left[\pi_{1} Z\right]}$. The second and third authors have done this for rings with involution in [LM, Appendix B] and we only need to adapt that to the present context.

We need to make one important change in the framework of [LM, Appendix B]. Expressions of the form $R \otimes_{R^{\mathrm{op}} \otimes R} N$ frequently occur there, where $N$ is an $R^{\mathrm{op}} \otimes R$ module. In corresponding situations in the present paper we will have a $\mathbb{Z}\left[\pi_{1} Z\right]^{\text {op }} \otimes \mathbb{Z}\left[\pi_{1} Z\right]$ module $\mathcal{N}$ and we will use the expression

$$
\mathcal{Z} \otimes_{\mathbb{Z}\left[\pi_{1} Z\right]} \mathcal{N}
$$

where $\mathcal{Z}$ is the constant right $\mathbb{Z}\left[\pi_{1} Z\right]$ module with value $\mathbb{Z}$ and we think of $\mathcal{N}$ as a left $\mathbb{Z}\left[\pi_{1} Z\right]$ module via the map

$$
\mathbb{Z}\left[\pi_{1} Z\right] \rightarrow \mathbb{Z}\left[\pi_{1} Z\right]^{\text {op }} \otimes \mathbb{Z}\left[\pi_{1} Z\right]
$$

which takes an object $z$ to $(z, z)$ and a path homotopy class $\delta$ to $\left(\delta^{-1}, \delta\right)$. (We could give a precise analogue of $R \otimes_{R^{\mathrm{op}} \otimes R} N$, but it would be isomorphic to $\mathcal{Z} \otimes_{\mathbb{Z}\left[\pi_{1} Z\right]} \mathcal{N}$ and the latter is easier to work with.) 
The first issue that must be addressed in creating $\Psi^{\prime \prime}$ is that, unfortunately, $\operatorname{ad}_{\mathrm{rel}}^{\mathbb{Z}\left[\pi_{1} Z\right]}$ isn't even "approximately" functorial, that is, given a map $g: Z \rightarrow Z^{\prime}$ and an object $(C, D, \beta, \phi)$ of $\mathcal{A}_{\mathrm{rel}}^{\mathbb{Z}\left[\pi_{1} Z\right]}$, there doesn't seem to be a reasonable way to create an object $\left(C^{\prime}, D^{\prime}, \beta^{\prime}, \phi^{\prime}\right)$ of $\mathcal{A}_{\mathrm{rel}}^{\mathbb{Z}\left[\pi_{1} Z^{\prime}\right]}$ (we could let $C^{\prime}=\operatorname{Kan}_{g_{*}} C$, but there's no reasonable candidate for $D^{\prime}$ ). [LM, Subsections B.1-B.4] construct an ad theory $\operatorname{ad}_{\mathrm{Rel}}^{R}$ which is approximately functorial (that is, functorial up to isomorphism) and has a map to $\mathrm{ad}_{\mathrm{rel}}^{R}$ which induces an isomorphism of bordism groups; in this subsection we generalize this.

[LM, Definition B.3 and Lemma B.4] generalize to our context in a straighforward way. The analogue of [LM, Definition B.5] is

Definition 10.15. A Relaxed quasi-symmetric complex of dimension $n$ over $\mathbb{Z}\left[\pi_{1} Z\right]$ is a quadruple $(C, E, \gamma, \phi)$, where $C$ is a homotopy finite chain complex over $\mathbb{Z}\left[\pi_{1} Z\right], E$ is a homotopy finite chain complex over $\mathbb{Z}\left[\pi_{1} Z\right]^{\text {op }} \otimes \mathbb{Z}\left[\pi_{1} Z\right]$ with a $\mathbb{Z} / 2$ action for which the generator acts quasilinearly, $\gamma$ is a $\mathbb{Z} / 2$ equivariant $\mathbb{Z}\left[\pi_{1} Z\right]^{\text {op }} \otimes \mathbb{Z}\left[\pi_{1} Z\right]$ linear quasi-isomorphism $C^{t} \otimes C \rightarrow E$, and $\phi$ is an $n$-dimensional element of $\left(\mathcal{Z} \otimes_{\mathbb{Z}\left[\pi_{1} Z\right]} E\right)^{\mathbb{Z} / 2}$.

Remark 10.16. $\left[\mathrm{LM}\right.$, Definition B.7] generalizes to give a category $\mathcal{A}_{\mathrm{Rel}}^{\mathbb{Z}\left[\pi_{1} Z\right]}$. Analogously to [LM, Remark B.8] we have a map

$$
\Lambda: \mathcal{A}_{\mathrm{Rel}}^{\mathbb{Z}\left[\pi_{1} Z\right]} \rightarrow \mathcal{A}_{\mathrm{rel}}^{\mathbb{Z}\left[\pi_{1} Z\right]}
$$

which takes $(C, E, \gamma, \phi)$ to $\left(C, \mathcal{Z} \otimes_{\mathbb{Z}\left[\pi_{1} Z\right]} E, \beta, \phi\right)$, where $\beta$ is the following composite (in which the isomorphism is Remark 7.5)

$$
C^{t} \otimes_{\mathbb{Z}\left[\pi_{1} Z\right]} C \cong \mathcal{Z} \otimes_{\mathbb{Z}\left[\pi_{1} Z\right]}\left(C^{t} \otimes C\right) \stackrel{1 \otimes \gamma}{\longrightarrow} \mathcal{Z} \otimes_{\mathbb{Z}\left[\pi_{1} Z\right]} E .
$$

[LM, Definitions B.10 and B.11] generalize to give a set $\operatorname{ad}_{\operatorname{Rel}}^{\mathbb{Z}\left[\pi_{1} Z\right]}(K)$ for each $K$; note that (as in [LM, Definition B.11(ii)]) every ad is isomorphic to a balanced ad. [LM, Theorem B.12] generalizes to the statement that this is an ad theory. The proof of [LM, Proposition B.17] generalizes to show that the map

$$
\Lambda: \operatorname{ad}_{\mathrm{Rel}}^{\mathbb{Z}\left[\pi_{1} Z\right]} \rightarrow \operatorname{ad}_{\mathrm{rel}}^{\mathbb{Z}\left[\pi_{1} Z\right]}
$$

induces an isomorphism of bordism groups.

Next we show that $\operatorname{ad}_{\operatorname{Rel}}^{\mathbb{Z}\left[\pi_{1} Z\right]}$ is approximately functorial.

Definition 10.17. Let $g: Z \rightarrow Z^{\prime}$ be a continuous function. Define a functor

$$
g_{\mathrm{Rel}}: \mathcal{A}_{\mathrm{Rel}}^{\mathbb{Z}\left[\pi_{1} Z\right]} \rightarrow \mathcal{A}_{\mathrm{Rel}}^{\mathbb{Z}\left[\pi_{1} Z^{\prime}\right]}
$$

as follows. For an object $(C, E, \gamma, \phi)$ of $\mathcal{A}_{\mathrm{Rel}}^{\mathbb{Z}\left[\pi_{1} Z\right]}$, let

$$
g_{\text {Rel }}\left((C, E, \gamma, \phi)=\left(\operatorname{Kan}_{g_{*}}(C), \operatorname{Kan}_{g_{*} \otimes g_{*}}(E), \gamma^{\prime}, \phi^{\prime}\right),\right.
$$


where $\gamma^{\prime}$ is the composite

$$
\left(\operatorname{Kan}_{g_{*}}(C)\right)^{t} \otimes \operatorname{Kan}_{g_{*}}(C) \cong \operatorname{Kan}_{g_{*} \otimes g_{*}}\left(C^{t} \otimes C\right) \stackrel{\operatorname{Kan}_{g_{*} \otimes g_{*} \gamma}}{\longrightarrow} \operatorname{Kan}_{g_{*} \otimes g_{*}}(E)
$$

(which is a quasi-isomorphism by Remarks 7.3 and B.3 and the Künneth spectral sequence [Weib94, Theorem 5.6.4]) and $\phi^{\prime}$ is the image of $\phi$ under the map

$$
\left(\mathcal{Z} \otimes_{\mathbb{Z}\left[\pi_{1} Z\right]} E\right)^{\mathbb{Z} / 2} \rightarrow\left(\mathcal{Z} \otimes_{\mathbb{Z}\left[\pi_{1} Z^{\prime}\right]} \operatorname{Kan}_{g_{*} \otimes g_{*}}(E)\right)^{\mathbb{Z} / 2}
$$

(where $\mathcal{Z}$ denotes the $\mathbb{Z}\left[\pi_{1} Z\right]$ module in the first expression and the $\mathbb{Z}\left[\pi_{1} Z^{\prime}\right]$ module in the second) which takes the class of $1 \otimes e$ (where $e \in E_{(z, z)}$ ) to the class of $1 \otimes \mathrm{id}_{(z, z)} \otimes e$.

The proof of [LM, Proposition B.15] generalizes (using Remarks 7.1, 7.2, 7.3, and B.3) to show that $g_{\text {Rel }}$ takes ads to ads.

10.5. The functor $\Psi^{\prime \prime}$. In this section we give a schematic version of

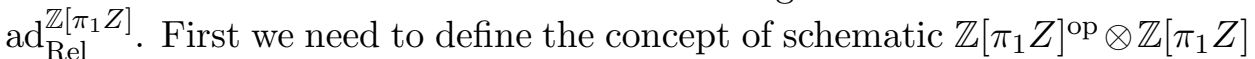
module.

There is an isomorphism

$$
\kappa: \mathbb{Z}\left[\pi_{1} Z\right]^{\text {op }} \otimes \mathbb{Z}\left[\pi_{1} Z\right] \cong \mathbb{Z}\left[\pi_{1}(Z \times Z)\right]
$$

which takes $\delta \otimes \delta^{\prime}$ to $\delta^{-1} \times \delta^{\prime}$, where $\delta$ and $\delta^{\prime}$ are path homotopy classes in $Z$.

Definition 10.18. (i) A schematic module over $\mathbb{Z}\left[\pi_{1} Z\right]^{\text {op }} \otimes \mathbb{Z}\left[\pi_{1} Z\right]$ is a schematic module, in the sense already defined, over $\mathbb{Z}\left[\pi_{1}(Z \times Z)\right]$.

(iii) For a schematic $\mathbb{Z}\left[\pi_{1} Z\right]^{\text {op }} \otimes \mathbb{Z}\left[\pi_{1} Z\right]$ module $\left(\left(z_{1}, z_{2}\right)\right.$, $\left.\mathbb{A}\right)$, define

$$
\left(\mathbb{Z}\left[\pi_{1} Z\right]^{\mathrm{op}} \otimes \mathbb{Z}\left[\pi_{1} Z\right]\right)\left\langle\left(z_{1}, z_{2}\right), \mathbb{A}\right\rangle
$$

to be the module induced from $\mathbb{Z}\left[\pi_{1}(Z \times Z)\right]\left\langle\left(z_{1}, z_{2}\right), \mathbb{A}\right\rangle$ by the isomorphism $\kappa$.

Definition 10.19. (i) A schematic Relaxed quasi-symmetric complex of dimension $n$ is a quadruple $(\mathbb{C}, \mathbb{E}, \gamma, \phi)$, where $\mathbb{C}$ is a schematic $\mathbb{Z}\left[\pi_{1} Z\right]$ chain complex, $\mathbb{E}$ is a schematic $\mathbb{Z}\left[\pi_{1} Z\right]^{\text {op }} \otimes \mathbb{Z}\left[\pi_{1} Z\right]$ chain complex, $\mathbb{Z}\left[\pi_{1} Z\right]\langle\mathbb{C}\rangle$ is homotopy finite, $\left(\mathbb{Z}\left[\pi_{1} Z\right]^{\text {op }} \otimes \mathbb{Z}\left[\pi_{1} Z\right]\right)\langle\mathbb{E}\rangle$ is a homotopy finite chain complex over $\mathbb{Z}\left[\pi_{1} Z\right]^{\text {op }} \otimes \mathbb{Z}\left[\pi_{1} Z\right]$ with a $\mathbb{Z} / 2$ action for which the generator acts quasi-linearly, $\gamma$ is a $\mathbb{Z} / 2$ equivariant quasi-isomorphism $\left(\mathbb{Z}\left[\pi_{1} Z\right]\langle\mathbb{C}\rangle\right)^{t} \otimes$ $\mathbb{Z}\left[\pi_{1} Z\right]\langle\mathbb{C}\rangle \rightarrow\left(\mathbb{Z}\left[\pi_{1} Z\right]^{\text {op }} \otimes \mathbb{Z}\left[\pi_{1} Z\right]\right)\langle\mathbb{E}\rangle$ of $\mathbb{Z}\left[\pi_{1} Z\right]^{\text {op }} \otimes \mathbb{Z}\left[\pi_{1} Z\right]$ chain complexes, and $\phi$ is an $n$-dimensional element of $\left(\mathcal{Z} \otimes_{\mathbb{Z}\left[\pi_{1} Z\right]}\left(\mathbb{Z}\left[\pi_{1} Z\right]^{\text {op }} \otimes \mathbb{Z}\left[\pi_{1} Z\right]\right)\langle\mathbb{E}\rangle\right)^{\mathbb{Z} / 2}$.

(ii) We define a category $\mathcal{A}_{\mathrm{Rel}, \text { sch }}^{\mathbb{Z}\left[\pi_{1} Z\right]}$ as follows. The objects of $\mathcal{A}_{\mathrm{Rel}, \mathrm{sch}}^{\mathbb{Z}\left[\pi_{1} Z\right]}$ are the schematic Relaxed quasi-symmetric complexes. A morphism $(\mathbb{C}, \mathbb{E}, \gamma, \phi) \rightarrow$ $\left(\mathbb{C}^{\prime}, \mathbb{E}^{\prime}, \gamma^{\prime}, \phi^{\prime}\right)$ is a pair $\left(f_{1}: \mathbb{Z}\left[\pi_{1} Z\right]\langle\mathbb{C}\rangle \rightarrow \mathbb{Z}\left[\pi_{1} Z\right]\left\langle\mathbb{C}^{\prime}\right\rangle, f_{2}:\left(\mathbb{Z}\left[\pi_{1} Z\right]^{\text {op }} \otimes\right.\right.$ $\left.\left.\mathbb{Z}\left[\pi_{1} Z\right]\right)\langle\mathbb{E}\rangle \rightarrow\left(\mathbb{Z}\left[\pi_{1} Z\right]^{\text {op }} \otimes \mathbb{Z}\left[\pi_{1} Z\right]\right)\left\langle\mathbb{E}^{\prime}\right\rangle\right)$, where $f_{1}$ is a map of $\mathbb{Z}\left[\pi_{1} Z\right]$ chain complexes and $f_{2}$ is a $\mathbb{Z} / 2$ equivariant map of $\mathbb{Z}\left[\pi_{1} Z\right]^{\text {op }} \otimes \mathbb{Z}\left[\pi_{1} Z\right]$ chain complexes, such that $f_{2} \gamma=\gamma^{\prime}\left(f_{1} \otimes f_{1}\right)$, and (if $\left.\operatorname{dim} \phi=\operatorname{dim} \phi^{\prime}\right)\left(1 \otimes f_{2}\right)_{*}(\phi)=\phi^{\prime}$. 
There is a functor

$$
\Theta_{\mathrm{Rel}}: \mathcal{A}_{\mathrm{Rel}, \mathrm{sch}}^{\mathbb{Z}\left[\pi_{1} Z\right]} \rightarrow \mathcal{A}_{\mathrm{Rel}}^{\mathbb{Z}\left[\pi_{1} Z\right]}
$$

which takes $(\mathbb{C}, \mathbb{E}, \gamma, \phi)$ to $\left(\mathbb{Z}\left[\pi_{1} Z\right]\langle\mathbb{C}\rangle,\left(\mathbb{Z}\left[\pi_{1} Z\right]^{\text {op }} \otimes \mathbb{Z}\left[\pi_{1} Z\right]\right)\langle\mathbb{E}\rangle, \gamma, \phi\right)$; this is an equivalence of categories.

Definition 10.20. A $K$-ad with values in $\mathcal{A}_{\mathrm{Rel}, \mathrm{sch}}^{\mathbb{Z}\left[\pi_{1} Z\right]}$ is a pre $K$-ad $F$ for which $\Theta_{\text {Rel }} \circ F$ is an ad.

The proof of [LM, Proposition B.22] shows that $\operatorname{ad}_{\mathrm{Rel}, \text { sch }}^{\mathbb{Z}\left[\pi_{1} Z\right]}$ is an ad theory and that $\Theta_{\text {Rel }}$ induces an isomorphism of bordism groups.

Next we consider functoriality. Let $g: Z \rightarrow Z^{\prime}$ be a continuous function.

Definition 10.21. Define a functor

$$
g_{\mathrm{Rel}, \mathrm{sch}}: \mathcal{A}_{\mathrm{Rel}, \mathrm{sch}}^{\mathbb{Z}\left[\pi_{1} Z\right]} \rightarrow \mathcal{A}_{\mathrm{Rel}, \mathrm{sch}}^{\mathbb{Z}\left[\pi_{1} Z^{\prime}\right]}
$$

as follows. For an object $(\mathbb{C}, \mathbb{E}, \gamma, \phi)$ of $\mathcal{A}_{\text {Rel,sch }}^{\mathbb{Z}\left[\pi_{1} Z\right]}$, let

$$
g_{\mathrm{Rel}, \mathrm{sch}}(\mathbb{C}, \mathbb{E}, \gamma, \phi)=\left(g_{\mathrm{sch}} \mathbb{C},(g \times g)_{\mathrm{sch}} \mathbb{E}, \delta, \psi\right),
$$

where $g_{\text {sch }}$ and $(g \times g)_{\text {sch }}$ are defined in Subsection 10.1 and (letting $C=\mathbb{Z}\left[\pi_{1} Z\right]\langle\mathbb{C}\rangle$ and $E=\left(\mathbb{Z}\left[\pi_{1} Z\right]^{\text {op }} \otimes \mathbb{Z}\left[\pi_{1} Z\right]\right)\langle\mathbb{E}\rangle$, and using the notation of Definitions 10.17) and 10.18(ii) and the isomorphism of Lemma 10.5) $\delta$ is defined by the diagram

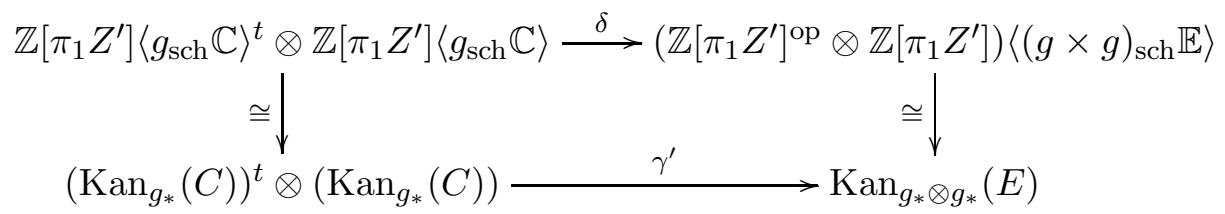

and $\psi$ is the image of $\phi^{\prime}$ under the isomorphism

$$
\begin{aligned}
\left(\mathcal{Z} \otimes_{\mathbb{Z}\left[\pi_{1} Z^{\prime}\right]} \operatorname{Kan}_{g_{*} \otimes g_{*}} E\right)^{\mathbb{Z} / 2} & \\
& \cong\left(\mathcal{Z} \otimes_{\mathbb{Z}\left[\pi_{1} Z^{\prime}\right]}\left(\mathbb{Z}\left[\pi_{1} Z^{\prime}\right]^{\text {op }} \otimes \mathbb{Z}\left[\pi_{1} Z^{\prime}\right]\right)\left\langle(g \times g)_{\text {sch }} \mathbb{E}\right\rangle\right)^{\mathbb{Z} / 2} .
\end{aligned}
$$

The proof of [LM, Proposition B.25] shows that $g_{\text {Rel,sch }}$ takes ads to ads. The reader can check that if $g^{\prime}: Z^{\prime} \rightarrow Z^{\prime \prime}$ is another continuous function then $\left(g^{\prime} g\right)_{\text {Rel,sch }}=g_{\text {Rel,sch }}^{\prime} g_{\text {Rel,sch }}$, so ad $\operatorname{Rel}_{\text {Rel,sch }}^{\mathbb{Z}\left[\pi_{1} Z\right]}$ is a functor of $Z$ as required.

We can now define $\Psi^{\prime \prime}$ by

$$
\Psi^{\prime \prime}(Z)=\mathbf{Q}_{\mathrm{Rel}, \mathrm{sch}, \geq 0}^{\mathbb{Z}\left[\pi_{1} Z\right]} .
$$

10.6. Background for the map $\Psi^{\prime} \rightarrow \Psi^{\prime \prime}$. Recall Remark 7.6. In the next subsection we will define $\Psi^{\prime}$ to be a certain schematic version of $\mathbf{Q}_{\mathrm{fin}}^{\mathbb{Z}\left[\pi_{1} Z\right]}$. In this subsection, as preparation for the construction of the map $\Psi^{\prime} \rightarrow \Psi^{\prime \prime}$, we give the nonschematic version of this map.

To do this, we will give a map

$$
\Pi: \mathcal{A}_{\mathrm{fin}}^{\mathbb{Z}\left[\pi_{1} Z\right]} \rightarrow \mathcal{A}_{\mathrm{Rel}}^{\mathbb{Z}\left[\pi_{1} Z\right]}
$$


(following [LM, Remarks B.6 and B.9]).

Recall Notation [10.6. For a chain complex $C$ over $\mathbb{Z}\left[\pi_{1} Z\right]$, let $\left(C^{t} \otimes C\right)^{W}$ be the chain complex over $\mathbb{Z}\left[\pi_{1} Z\right]^{\text {op }} \otimes \mathbb{Z}\left[\pi_{1} Z\right]$ with

$$
\left(\left(C^{t} \otimes C\right)^{W}\right)_{\left(z_{1}, z_{2}\right)}=\left(C_{z_{1}}^{t} \otimes C_{z_{2}}\right)^{W}
$$

When $C$ is finite, $\left(C^{t} \otimes C\right)^{W}$ is (additively) a direct sum of copies of $C^{t} \otimes C$, and hence the natural map

$$
\mathcal{Z} \otimes_{\mathbb{Z}\left[\pi_{1} Z\right]}\left(\left(C^{t} \otimes C\right)^{W}\right) \rightarrow\left(\mathcal{Z} \otimes_{\mathbb{Z}\left[\pi_{1} Z\right]}\left(C^{t} \otimes C\right)\right)^{W}
$$

is an isomorphism because tensor product preserves direct sums.

Now define $\Pi$ by

$$
\Pi(C, \varphi)=\left(C,\left(C^{t} \otimes C\right)^{W}, \gamma, \phi\right),
$$

where $\gamma: C^{t} \otimes C \rightarrow\left(C^{t} \otimes C\right)^{W}$ is induced by the augmentation $W \rightarrow \mathbb{Z}$ and $\phi$ is the image of $\varphi$ under the composite

$$
\begin{aligned}
\left(\left(C^{t} \otimes C\right)^{W}\right)^{\mathbb{Z} / 2} \cong\left(\left(\mathcal{Z} \otimes_{\mathbb{Z}\left[\pi_{1} Z\right]}\left(C^{t} \otimes C\right)\right)^{W}\right)^{\mathbb{Z} / 2} & \\
& \cong\left(\mathcal{Z} \otimes_{\mathbb{Z}\left[\pi_{1} Z\right]}\left(\left(C^{t} \otimes C\right)^{W}\right)\right)^{\mathbb{Z} / 2}
\end{aligned}
$$

(where the first isomorphism is Remark 7.5).

$\Pi$ takes ads to ads, and the proof of [LM, Proposition B.17] shows that it induces an isomorphism of bordism groups.

Remark 10.22. Consider the following diagram, where the left vertical arrow was given in Remark 7.6, the map $\Lambda$ in Remark 10.16, and the lower horizontal map in Remark 8.1;

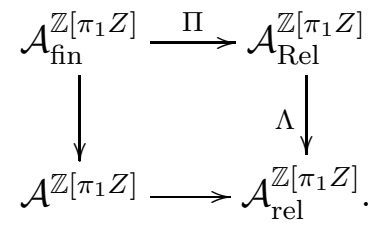

The diagram commutes up to natural isomorphism, so the following diagram homotopy commutes, by the analog of [LM, Proposition 14.5].

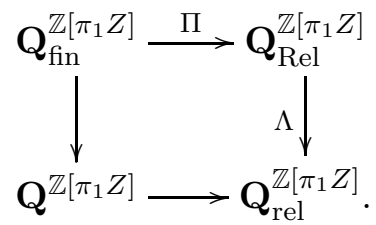

10.7. The functor $\Psi^{\prime}$ and the map $\Psi^{\prime} \rightarrow \Psi$. Our first task is to give a suitable schematic version $\mathcal{A}_{\mathrm{fin}, \mathrm{sch}}^{\mathbb{Z}\left[\pi_{1} Z\right]}$ of $\mathcal{A}_{\mathrm{fin}}^{\mathbb{Z}\left[\pi_{1} Z\right]}$. The obvious choice would be the subcategory of $\mathcal{A}_{\mathrm{sch}}^{\mathbb{Z}\left[\pi_{1} Z\right]}$ consisting of objects $(\mathbb{C}, \varphi)$ for which $\mathbb{Z}\left[\pi_{1} Z\right]\langle\mathbb{C}\rangle$ is finite, but there is no reasonable way to give a map from this to $\mathcal{A}_{\mathrm{Rel}, \mathrm{sch}}^{\mathbb{Z}\left[\pi_{1} Z\right.}$; the difficulty is that given an object $(\mathbb{C}, \varphi)$ there is no reasonable choice for 
$\mathbb{E}$. We will therefore build a choice for $\mathbb{E}$ into the definition of the category $\mathcal{A}_{\text {fin,sch }}^{\mathbb{Z}\left[\pi_{1} Z\right]}$.

Definition 10.23. Let $C$ be a chain complex over $\mathbb{Z}\left[\pi_{1} Z\right]$ (resp., $\mathbb{Z}\left[\pi_{1} Z\right]^{\text {op }} \otimes$ $\left.\mathbb{Z}\left[\pi_{1} Z\right]\right)$. A schematic model for $C$ is a pair $(\mathbb{C}, \lambda)$, where $\mathbb{C}$ is a schematic chain complex over $\mathbb{Z}\left[\pi_{1} Z\right]$ (resp., $\mathbb{Z}\left[\pi_{1} Z\right]^{\text {op }} \otimes \mathbb{Z}\left[\pi_{1} Z\right]$ ) and $\lambda$ is an isomorphism $\mathbb{Z}\left[\pi_{1} Z\right]\langle\mathbb{C}\rangle \rightarrow C$ (resp., $\left.\left(\mathbb{Z}\left[\pi_{1} Z\right]^{\text {op }} \otimes \mathbb{Z}\left[\pi_{1} Z\right]\right)\langle\mathbb{C}\rangle \rightarrow C\right)$.

Definition 10.24. Define a category $\mathcal{A}_{\text {fin,sch }}^{\mathbb{Z}\left[\pi_{1} Z\right]}$ as follows. An object is a quadruple

$$
(\mathbb{C}, \varphi, \mathbb{E}, \lambda)
$$

where $(\mathbb{C}, \varphi)$ is an object of $\mathcal{A}_{\mathrm{sch}}^{\mathbb{Z}\left[\pi_{1} Z\right]}$ with $\mathbb{Z}\left[\pi_{1} Z\right]\langle\mathbb{C}\rangle$ finite and $(\mathbb{E}, \lambda)$ is a schematic model for $\left(\left(\mathbb{Z}\left[\pi_{1} Z\right]\langle\mathbb{C}\rangle\right)^{t} \otimes \mathbb{Z}\left[\pi_{1} Z\right]\langle\mathbb{C}\rangle\right)^{W}$. The morphisms $(\mathbb{C}, \varphi, \mathbb{E}, \lambda) \rightarrow\left(\mathbb{C}^{\prime}, \varphi^{\prime}, \mathbb{E}^{\prime}, \lambda^{\prime}\right)$ are the morphisms $(\mathbb{C}, \varphi) \rightarrow\left(\mathbb{C}^{\prime}, \varphi^{\prime}\right)$ in $\mathcal{A}_{\mathrm{sch}}^{\mathbb{Z}\left[\pi_{1} Z\right]}$.

Note that the definition of morphism does not involve the schematic model $(\mathbb{E}, \lambda)$. Because of this, the functor

$$
\Theta_{\text {fin }}: \mathcal{A}_{\text {fin,sch }}^{\mathbb{Z}\left[\pi_{1} Z\right]} \rightarrow \mathcal{A}_{\text {fin }}^{\mathbb{Z}\left[\pi_{1} Z\right]}
$$

which takes $(\mathbb{C}, \varphi, \mathbb{E}, \lambda)$ to $\left(\mathbb{Z}\left[\pi_{1} Z\right]\langle\mathbb{C}\rangle, \varphi\right)$ is an equivalence of categories.

Definition 10.25. An ad with values in $\mathcal{A}_{\text {fin,sch }}^{\mathbb{Z}\left[\pi_{1} Z\right]}$ is a pread whose composite with $\Theta_{\text {fin }}$ is an ad.

The proof of Proposition 10.9 shows that this gives an ad theory and that the map $\operatorname{ad}_{\mathrm{fin}, \mathrm{sch}}^{\mathbb{Z}\left[\pi_{1} Z\right]} \rightarrow \operatorname{ad}_{\mathrm{fin}}^{\mathbb{Z}\left[\pi_{1} Z\right]}$ induces an isomorphism of bordism groups.

Next we show that $\operatorname{ad}_{\mathrm{fin}, \mathrm{sch}}^{\mathbb{Z}\left[\pi_{1} Z\right]}$ is a functor of $Z$. Let $g: Z \rightarrow Z^{\prime}$ be a continuous map, and define a functor

$$
g_{\text {fin }, \text { sch }}: \mathcal{A}_{\text {fin }, \mathrm{sch}}^{\mathbb{Z}\left[\pi_{1} Z\right]} \rightarrow \mathcal{A}_{\mathrm{fin}, \mathrm{sch}}^{\mathbb{Z}\left[\pi_{1} Z^{\prime}\right]}
$$

by

$$
g_{\text {fin }, \text { sch }}(\mathbb{C}, \varphi, \mathbb{E}, \lambda)=\left(g_{\text {sch }}^{\text {sym }}(\mathbb{C}, \varphi),(g \times g)_{\text {sch }} \mathbb{E}, \lambda^{\prime}\right)
$$

where $g_{\text {sch }}^{\text {sym }}$ was defined in Subsection 10.2 and $\lambda^{\prime}$ is the following composite (where the first isomorphism is given by Lemma 10.5)

$$
\begin{gathered}
\left(\mathbb{Z}\left[\pi_{1} Z^{\prime}\right]^{\text {op }} \otimes \mathbb{Z}\left[\pi_{1} Z^{\prime}\right]\right)\left\langle(g \times g)_{\text {sch }} \mathbb{E}\right\rangle \cong \operatorname{Kan}_{g_{*} \otimes g_{*}}\left(\left(\mathbb{Z}\left[\pi_{1} Z\right]^{\text {op }} \otimes \mathbb{Z}\left[\pi_{1} Z\right]\right)\langle\mathbb{E}\rangle\right) \\
\stackrel{\operatorname{Kan}_{g_{*} \otimes g_{*}}(\lambda)}{\longrightarrow} \operatorname{Kan}_{g_{*} \otimes g_{*}}\left(\left(\left(\mathbb{Z}\left[\pi_{1} Z\right]\langle\mathbb{C}\rangle\right)^{t} \otimes \mathbb{Z}\left[\pi_{1} Z\right]\langle\mathbb{C}\rangle\right)^{W}\right) \\
\cong\left(\operatorname{Kan}_{g_{*} \otimes g_{*}}\left(\left(\mathbb{Z}\left[\pi_{1} Z\right]\langle\mathbb{C}\rangle\right)^{t} \otimes \mathbb{Z}\left[\pi_{1} Z\right]\langle\mathbb{C}\rangle\right)\right)^{W} \\
\cong\left(\left(\mathbb{Z}\left[\pi_{1} Z^{\prime}\right]\left\langle g_{\text {sch }} \mathbb{C}\right\rangle\right)^{t} \otimes \mathbb{Z}\left[\pi_{1} Z^{\prime}\right]\left\langle g_{\text {sch }} \mathbb{C}\right\rangle\right)^{W}
\end{gathered}
$$

$g_{\mathrm{fin}, \mathrm{sch}}$ takes ads to ads by Proposition 10.11, We leave it to the reader to check that if $g^{\prime}: Z^{\prime} \rightarrow Z^{\prime \prime}$ then $\left(g^{\prime} g\right)_{\text {fin }, \text { sch }}=g_{\text {fin }, \text { sch }}^{\prime} g_{\text {fin }, \text { sch }}$.

Now we can define

$$
\Psi^{\prime}(Z)=\mathbf{Q}_{\mathrm{fin}, \mathrm{sch}, \geq 0}^{\mathbb{Z}\left[\pi_{1} Z\right]}
$$


The functor

$$
\Psi^{\prime} \rightarrow \Psi
$$

is induced by the map

$$
\mathcal{A}_{\mathrm{fin}, \mathrm{sch}}^{\mathbb{Z}\left[\pi_{1} Z\right]} \rightarrow \mathcal{A}_{\mathrm{sch}}^{\mathbb{Z}\left[\pi_{1} Z\right]}
$$

which takes $(\mathbb{C}, \varphi, \mathbb{E}, \lambda)$ to $(\mathbb{C}, \varphi)$.

10.8. The map $\Psi^{\prime} \rightarrow \Psi^{\prime \prime}$. In order to define the map $\Psi^{\prime} \rightarrow \Psi^{\prime \prime}$, we first define

as follows.

$$
\Pi_{\mathrm{sch}}: \mathcal{A}_{\mathrm{fin}, \mathrm{sch}}^{\mathbb{Z}\left[\pi_{1} Z\right]} \rightarrow \mathcal{A}_{\mathrm{Rel}, \mathrm{sch}}^{\mathbb{Z}\left[\pi_{1} Z\right]}
$$

On objects, $\Pi_{\text {sch }}$ is defined by

$$
\Pi_{\mathrm{sch}}(\mathbb{C}, \varphi, \mathbb{E}, \lambda)=(\mathbb{C}, \mathbb{E}, \gamma, \phi) ;
$$

here $\gamma$ is the composite

$$
\begin{aligned}
&\left(\mathbb{Z}\left[\pi_{1} Z\right]\langle\mathbb{C}\rangle\right)^{t} \otimes \mathbb{Z}\left[\pi_{1} Z\right]\langle\mathbb{C}\rangle \rightarrow\left(\left(\mathbb{Z}\left[\pi_{1} Z\right]\langle\mathbb{C}\rangle\right)^{t} \otimes \mathbb{Z}\left[\pi_{1} Z\right]\langle\mathbb{C}\rangle\right)^{W} \\
& \stackrel{\lambda^{-1}}{\longrightarrow}\left(Z\left[\pi_{1} Z\right]^{\text {op }} \otimes \mathbb{Z}\left[\pi_{1} Z\right]\right)\langle\mathbb{E}\rangle,
\end{aligned}
$$

(where the first map is induced by the augmentation $W \rightarrow \mathbb{Z}$ ), and (writing $C$ for $\mathbb{Z}\left[\pi_{1} Z\right]\langle\mathbb{C}\rangle$ and $E$ for $\left.\left(Z\left[\pi_{1} Z\right]^{\text {op }} \otimes \mathbb{Z}\left[\pi_{1} Z\right]\right)\langle\mathbb{E}\rangle\right) \phi$ is the image of $\varphi$ under the composite

$$
\begin{aligned}
& \left(\left(C^{t} \otimes C\right)^{W}\right)^{\mathbb{Z} / 2} \cong\left(\left(\mathcal{Z} \otimes_{\mathbb{Z}\left[\pi_{1} Z\right]}\left(C^{t} \otimes C\right)\right)^{W}\right)^{\mathbb{Z} / 2} \\
& \cong\left(\mathcal{Z} \otimes_{\mathbb{Z}\left[\pi_{1} Z\right]}\left(\left(C^{t} \otimes C\right)^{W}\right)\right)^{\mathbb{Z} / 2} \stackrel{1 \otimes \lambda^{-1}}{\longrightarrow}\left(\mathcal{Z} \otimes_{\mathbb{Z}\left[\pi_{1} Z\right]} E\right)^{\mathbb{Z} / 2}
\end{aligned}
$$

(where the first isomorphism is Remark 7.5).

To define $\Pi_{\text {sch }}$ on morphisms, recall that a morphism $(\mathbb{C}, \varphi, \mathbb{E}, \lambda) \rightarrow$ $\left(\mathbb{C}^{\prime}, \varphi^{\prime}, \mathbb{E}^{\prime}, \lambda^{\prime}\right)$ is just a map of chain complexes $f: \mathbb{Z}\left[\pi_{1} Z\right]\langle\mathbb{C}\rangle \rightarrow \mathbb{Z}\left[\pi_{1} Z\right]\left\langle\mathbb{C}^{\prime}\right\rangle$ (such that $(f \otimes f) \circ \varphi=\varphi^{\prime}$ if $\left.\operatorname{dim} \varphi=\operatorname{dim} \varphi^{\prime}\right)$. We define $\Pi_{\text {sch }}$ of such a morphism to be the morphism $\left(f, f_{1}\right)$, where $f_{1}$ is the composite

$$
\begin{aligned}
\left(Z\left[\pi_{1} Z\right]^{\mathrm{op}} \otimes \mathbb{Z}\left[\pi_{1} Z\right]\right)\langle\mathbb{E}\rangle \stackrel{\lambda}{\rightarrow}\left(\left(Z\left[\pi_{1} Z\right]\langle\mathbb{C}\rangle\right)^{t} \otimes Z\left[\pi_{1} Z\right]\langle\mathbb{C}\rangle\right)^{W} \\
\stackrel{(f \otimes f)^{W}}{\longrightarrow}\left(\left(Z\left[\pi_{1} Z\right]\left\langle\mathbb{C}^{\prime}\right\rangle\right)^{t} \otimes Z\left[\pi_{1} Z\right]\left\langle\mathbb{C}^{\prime}\right\rangle\right)^{W} \\
\stackrel{\left(\lambda^{\prime}\right)^{-1}}{\longrightarrow}\left(Z\left[\pi_{1} Z\right]^{\text {op }} \otimes \mathbb{Z}\left[\pi_{1} Z\right]\right)\left\langle\mathbb{E}^{\prime}\right\rangle .
\end{aligned}
$$

To see that $\Pi_{\text {sch }}$ takes ads to ads, consider the diagram

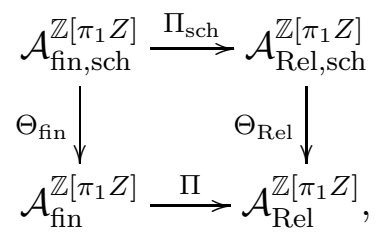

where $\Theta_{\text {fin }}$ was defined in Subsection 10.7, $\Theta_{\text {Rel }}$ in Subsection 10.5, and $\Pi$ in Subsection 10.6. The diagram commutes up to natural isomorphism. We 
need to show that if $F$ is an ad with values in $\mathcal{A}_{\text {fin,sch }}^{\mathbb{Z}\left[\pi_{1} Z\right]}$ then $\Pi_{\text {sch }} \circ F$ is an ad, and for this it suffices by Definition 10.20 to show that $\Theta_{\operatorname{Rel}} \circ \Pi_{\text {sch }} \circ F$ is an ad, and for this in turn it suffices to show that $\Pi \circ \Theta_{\text {fin }} \circ F$ is an ad. But $\Theta_{\text {fin }} \circ F$ is an ad by Definition 10.25 , and $\Pi$ takes ads to ads.

Now we can define the map

$$
\Psi^{\prime} \rightarrow \Psi^{\prime \prime}
$$

to be the map

induced by $\Pi_{\text {sch }}$.

$$
\mathbf{Q}_{\mathrm{fin}, \mathrm{sch}, \geq 0}^{\mathbb{Z}\left[\pi_{1} Z\right]} \rightarrow \mathbf{Q}_{\mathrm{Rel}, \mathrm{sch}, \geq 0}^{\mathbb{Z}\left[\pi_{1} Z\right]}
$$

To see that this map is an equivalence, we only need to observe that in the diagram above the two vertical maps and the lower horizontal map all induce isomorphisms of bordism groups.

10.9. Background for $\Phi^{\prime}$. Recall that $\Phi(Z)=\mathbf{Q}_{\mathrm{IP}, Z}$. We need to create a functor $\Phi^{\prime}$ which is equivalent to $\Phi$ and has a symmetric signature map to $\Psi^{\prime \prime}$. For this we need a suitable ad theory for each $Z$, and for this in turn we need a category $\mathcal{A}$ for each $Z$. We have seen in Subsection 6.1 that $\mathbf{Q}_{\text {IPFun }}$ is equivalent to $\mathbf{Q}_{\mathrm{IP}}$ and has a symmetric signature map to $\mathbf{Q}_{\mathrm{rel}, \geq 0}^{\mathbb{Z}}$, which suggests that we could let the objects of $\mathcal{A}$ be the pairs $(f: X \rightarrow Z, \xi)$, where $\xi$ is a representative for the fundamental class. However, this does not map to $\mathcal{A}_{\text {Rel,sch }}^{\mathbb{Z}\left[\pi_{1} Z\right]}$, because an object of $\mathcal{A}_{\text {Rel,sch }}^{\mathbb{Z}\left[\pi_{1} Z\right]}$ has the form $(\mathbb{C}, \mathbb{E}, \gamma, \phi)$ and a pair $(f: X \rightarrow Z, \xi)$ does not give a canonical choice for $\mathbb{C}$ and $\mathbb{E}$. This suggests that, as in Subsection 10.7, we should include schematic models $\left(\mathbb{C}, \lambda_{1}\right)$ and $\left(\mathbb{E}, \lambda_{2}\right)$ as part of the structure of an object of $\mathcal{A}$ (we'll say later what these are models of). Unfortunately, this still isn't functorial (cf. the proof of Lemma 10.33(i)). For functoriality, we need to add another ingredient, namely the intersection chains that are compatible with a certain open cover of $X$.

In this subsection we work out the theory of this additional ingredient, and in the next subsection we combine this with the other ingredients to create $\Phi^{\prime}$.

First we need some terminology and notation.

Let $\mathcal{U}$ be an open covering of a space $X$. For each perversity $\bar{p}$ we write $I S_{*}^{\bar{p}, \mathcal{U}}(X ; \mathbb{Z})$ for the subcomplex of $I S_{*}^{\bar{p}}(X ; \mathbb{Z})$ generated by the subcomplexes $I S_{*}^{\bar{p}}(U ; \mathbb{Z})$ with $U \in \mathcal{U}$ (this is somewhat different from the notation in [FM13b, Section 6]).

Remark 10.26. [F07, Proposition 2.9] shows that the inclusion

$$
I S_{*}^{\bar{p}, \mathcal{U}}(X ; \mathbb{Z}) \rightarrow I S_{*}^{\bar{p}}(X ; \mathbb{Z})
$$

is a quasi-isomorphism.

Definition 10.27. Let $f: X \rightarrow Z$ be an object of $\mathcal{A}_{\mathrm{IP}, Z}$. The open cover $\mathcal{U}_{X}$ of $X$ consists of the contractible open sets. 
Define a category $\mathcal{A}_{\mathrm{IP}, Z \text {,Fun }}$ as follows. The objects are pairs $(f: X \rightarrow$ $Z, \xi)$, where $f$ is an object of $\mathcal{A}_{\mathrm{IP}, Z}$ and $\xi$ is an element of $I S_{*}^{\overline{0}} \mathcal{U}_{X}(X ; \mathbb{Z})$ whose image in $I S_{*}^{\overline{0}}(X, \partial X ; \mathbb{Z})$ represents the fundamental class $\Gamma_{X} \in$ $I H_{n}^{\overline{0}}(X, \partial X ; \mathbb{Z})$; there is also an empty object of dimension $n$ for each $n$. The morphisms $(f: X \rightarrow Z, \xi) \rightarrow\left(f^{\prime}: X^{\prime} \rightarrow Z, \xi^{\prime}\right)$ are morphisms $g: X \rightarrow X^{\prime}$ in $\mathcal{A}_{\mathrm{IP}, Z}$ which, if the dimensions are equal, have the property that $g_{*} \xi=\xi^{\prime}$.

Definition 10.28. Define $\operatorname{ad}_{\mathrm{IP}, Z, F u n}(K) \subset \operatorname{pre}_{\mathrm{IP}, Z, F u n}^{k}(K)$ to be the set of functors $F$ such that

(a) $F$ is balanced,

(b) the composite of $F$ with the forgetful functor $\mathcal{A}_{\mathrm{IP}, Z \text {,Fun }} \rightarrow \mathcal{A}_{\mathrm{IP}}$ is an element of $\operatorname{ad}_{\mathrm{IP}}^{k}(K)$, and

(c) for each oriented cell $(\sigma, o)$ of $K$, the equation

$$
\partial \xi_{\sigma, o}=\sum \xi_{\sigma^{\prime}, o^{\prime}}
$$

holds, where $\sigma^{\prime}$ runs through the cells of $\partial \sigma$ and $o^{\prime}$ is the orientation for which the incidence number $\left[o, o^{\prime}\right]$ is $(-1)^{k}$.

Proposition 10.29. $\operatorname{ad}_{\mathrm{IP}, Z, F u n}$ is a connective ad theory.

Proof. We only need to check parts (f) and (g) of [LM13, Definition 3.10].

For the proof of (f), we may assume that $K$ and $K^{\prime}$ are as in the corresponding part of the proof of Theorem 4.4. Let $F$ be a $K^{\prime}$-ad and write

$$
F(\sigma, o)=\left(f_{\sigma}: X_{\sigma, o} \rightarrow Z, \xi_{(\sigma, o)}\right) .
$$

Let $Y_{\sigma}$ be the underlying $\partial$-IP-space of $X_{\sigma, o}$ (forgetting the orientation). Let

$$
Y_{\tau}=\operatorname{colim}_{\sigma \in K^{\prime}} Y_{\sigma}
$$

The proof of Theorem 4.4 shows that $Y_{\tau}$ is a $\partial$-IP-space and that an orientation $o$ of $\tau$ determines an orientation of $Y_{\tau}$; we let $X_{\tau, o}$ be $Y_{\tau}$ with this orientation. Let $f_{\tau}: X_{\tau, o} \rightarrow Z$ be the map induced by the $f_{\sigma}$. As in the proof of Proposition 6.2, let

$$
\xi_{\tau, o}=\sum_{\sigma \in K^{\prime}} \xi_{\sigma, o_{\sigma}}
$$

$\xi_{\tau, o}$ is in $I S_{*}^{\overline{0}, \mathcal{U}_{X_{\tau, o}}}\left(X_{\tau, o} ; \mathbb{Z}\right)$, because by Lemma 6.7 every contractible open set of $X_{\sigma, o_{\sigma}}$ is contained in a contractible open subset of $X_{\tau, o}$. The desired result now follows from the proofs of Theorem 4.4 and Proposition 6.2.

For the proof of (g), let $F$ be a $K$-ad, and write

$$
F(\sigma, o)=\left(f_{\sigma}: X_{\sigma, o} \rightarrow Z, \xi_{\sigma, o}\right) .
$$

As in the proof of Proposition 6.2, we only need to specify $J(F)$ for oriented cells of the form $\left(\sigma \times I, o \times o^{\prime}\right)$ where $o^{\prime}$ is the standard orientation. Let

$$
g_{\sigma}: X_{\sigma, o} \times I \rightarrow Z
$$


be the composite of $f_{\sigma}$ with the projection. Let $s: \Delta^{1} \rightarrow I$ be the standard oriented homeomorphism. Recall the relative barycentric subdivision construction from [Mu84, Section 16]. For each $\sigma$ we can apply iterated barycentric subdivision to $\xi_{\sigma, o} \times s$, while holding fixed any faces that land in $X_{\sigma, o} \times\{0,1\}$, to obtain an element $\eta_{\sigma, o} \in I S^{\overline{0}, \mathcal{U}_{X_{\sigma, o}}}\left(X_{\sigma, o} \times I, \mathbb{Z}\right)$. Now let

$$
J(F)\left(\sigma \times I, o \times o^{\prime}\right)=\left(g_{\sigma}: X_{\sigma, o} \times I \rightarrow Z, \eta_{\sigma, o}\right) .
$$

The forgetful functor

$$
\Upsilon: \mathcal{A}_{\mathrm{IP}, Z, \mathrm{Fun}} \rightarrow \mathcal{A}_{\mathrm{IP}, Z}
$$

gives a morphism adIP, $Z$,Fun $\rightarrow \operatorname{ad}_{\mathrm{IP}, Z}$ of ad theories.

Proposition 10.30. The map

$$
\mathrm{Q}_{\Upsilon}: \mathrm{Q}_{\mathrm{IP}, Z, \text { Fun }} \rightarrow \mathrm{Q}_{\mathrm{IP}, Z}
$$

induced by $\Upsilon$ is a weak equivalence.

Proof. We need to show that the map of bordism groups

$$
\Omega_{\Upsilon}:\left(\Omega_{\mathrm{IP}, Z, F u n}\right)_{*} \rightarrow\left(\Omega_{\mathrm{IP}, Z}\right)_{*}
$$

is an isomorphism.

To see that it is onto, let $f: X \rightarrow Z$ be an element of $\operatorname{ad}_{\mathrm{IP}, Z}(*)$. Let $\xi \in$ $I S^{\overline{0}}(X ; \mathbb{Z})$ represent the fundamental class, and apply iterated barycentric subdivision to $\xi$ to get an element $\eta \in I S_{*}^{\overline{0}}, \mathcal{U}_{X}(X ; \mathbb{Z})$. Then $f: X \rightarrow Z$ is equal to $\Upsilon(f: X \rightarrow Z, \eta)$.

Next we claim that if $\left(f: X \rightarrow Z, \xi_{1}\right)$ and $\left(f: X \rightarrow Z, \xi_{2}\right)$ are elements of $\operatorname{ad}_{I P, Z, F u n}(*)$ with the same $f$ then they represent the same element of $\left(\Omega_{\mathrm{IP}, Z, \text { Fun }}\right)_{*}$. Let $g: X \times I \rightarrow Z$ be the composite of $f$ with the projection. The proof of [LM13, Lemma 8.2] gives an element $\eta \in I S^{\overline{0}}(X \times I ; \mathbb{Z})$ with $\partial \eta=\xi_{2}-\xi_{1}$. Applying iterated barycentric subdivision, holding fixed any faces that land in $X \times\{0,1\}$, gives an element $\eta^{\prime} \in I S^{\overline{0}, \mathcal{U}_{X \times I}}(X \times I ; \mathbb{Z})$ with $\partial \eta^{\prime}=\xi_{2}-\xi_{1}$. Now let $F$ be the $I$-ad which takes the cells 0,1 and $I$ (with their standard orientations) respectively to $\left(f, \xi_{1}\right),\left(f, \xi_{2}\right)$, and $\left(g, \eta^{\prime}\right)$; then $F$ is the desired bordism.

Now to see that $\Omega_{\Upsilon}$ is $1-1$, let $\left(f_{1}: X_{1} \rightarrow Z, \xi_{1}\right)$ and $\left(f_{2}: X_{2} \rightarrow Z, \xi_{2}\right)$ be elements of $\operatorname{ad}_{\mathrm{IP}, Z, F u n}(*)$, and let $F \in \operatorname{ad}_{\mathrm{IP}, Z}(I)$ be an ad which takes 0 and 1 (with their standard orientations) to $f_{1}$ and $f_{2}$. Write $g: Y \rightarrow Z$ for the image of $I$ (with its standard orientation) under $F$. Let $\eta \in I S^{\overline{0}}(Y ; \mathbb{Z})$ be a representative of the fundamental class of $Y$, and apply iterated barycentric subdivision to get an element $\eta^{\prime} \in I S^{\overline{0}, \mathcal{U}_{Y}}(Y ; \mathbb{Z})$. Then we can write

$$
\partial \eta^{\prime}=\theta_{1}-\theta_{2}
$$

where $\theta_{i} \in I S^{\overline{0}, \mathcal{U}_{X_{i}}}\left(X_{i} ; \mathbb{Z}\right)$ is a representative for the fundamental class of $X_{i}$. Now we have an ad $G$ in $\operatorname{ad}_{\mathrm{IP}, Z, F u n}(I)$ which takes 0,1 and $I$ (with their standard orientations) respectively to $\left(f_{1}: X_{1} \rightarrow Z, \theta_{1}\right),\left(f_{2}: X_{2} \rightarrow Z, \theta_{2}\right)$, 
and $\left(f: Y \rightarrow Z, \eta^{\prime}\right)$. This shows that $\left(f_{1}: X_{1} \rightarrow Z, \theta_{1}\right)$ and $\left(f_{2}: X_{2} \rightarrow Z, \theta_{2}\right)$ represent the same element of $\left(\Omega_{\mathrm{IP}, Z, F u n}\right)_{*}$, and by the previous paragraph we see that $\left(f_{1}: X_{1} \rightarrow Z, \xi_{1}\right)$ and $\left(f_{2}: X_{2} \rightarrow Z, \xi_{2}\right)$ represent the same element of $\left(\Omega_{\mathrm{IP}, Z, \mathrm{Fun}}\right)_{*}$.

Remark 10.31. We make $\mathcal{A}_{\mathrm{IP}, Z, \text { Fun }}$ a functor of $Z$ by letting $g: Z \rightarrow W$ take $(f: X \rightarrow Z, \xi)$ to $(g \circ f: X \rightarrow W, \xi)$; this takes ads to ads by Definition 10.28 .

10.10. The functor $\Phi^{\prime}$. We need a preliminary definition.

Definition 10.32. Let $f: X \rightarrow Z$ be an object of $\mathcal{A}_{\mathrm{IP}, Z}$.

(i) For each $z \in Z$, let $\tilde{Z}_{z} \rightarrow Z$ be the universal cover consisting of path homotopy classes of paths starting at $z$.

(ii) Let $C_{f: X \rightarrow Z}$ be the chain complex of left $\mathbb{Z}\left[\pi_{1} Z\right]$ modules with

$$
\left(C_{f: X \rightarrow Z}\right)_{z}=I S_{*}^{\bar{n}, \mathcal{U}_{f^{*}\left(\tilde{Z}_{z}\right)}}\left(f^{*}\left(\tilde{Z}_{z}\right) ; \mathbb{Z}\right)
$$

(iii) Let $E_{f: X \rightarrow Z}$ be the chain complex of left $\mathbb{Z}\left[\pi_{1} Z\right]^{\text {op }} \otimes \mathbb{Z}\left[\pi_{1} Z\right]$ modules with

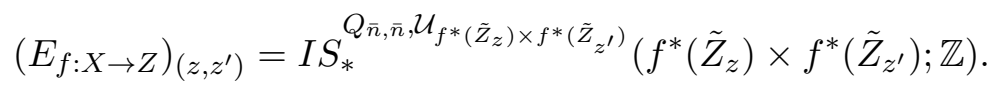

Now define a category $\mathcal{A}_{\mathrm{IP}, Z \text {,Fun,sch }}$ as follows. The objects are sextuples

$$
\left(f: X \rightarrow Z, \xi, \mathbb{C}, \lambda_{1}, \mathbb{E}, \lambda_{2}\right),
$$

where $(f: X \rightarrow Z, \xi)$ is an object of $\mathcal{A}_{\mathrm{IP}, Z, \text { Fun }},\left(\mathbb{C}, \lambda_{1}\right)$ is a schematic model

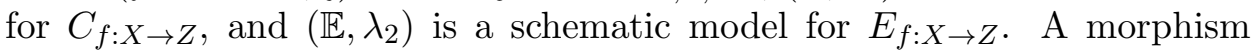
$(f: X \rightarrow Z, \xi, \mathbb{C}, \kappa, \mathbb{E}, \lambda) \rightarrow\left(f^{\prime}: X^{\prime} \rightarrow Z, \xi^{\prime}, \mathbb{C}^{\prime}, \kappa^{\prime}, \mathbb{E}^{\prime}, \lambda^{\prime}\right)$ is a morphism $(f: X \rightarrow Z, \xi) \rightarrow\left(f^{\prime}: X^{\prime} \rightarrow Z, \xi^{\prime}\right)$ in $\mathcal{A}_{\mathrm{IP}, Z \text {,Fun }}$.

There is a forgetful functor $\mathcal{A}_{\mathrm{IP}, Z \text {,Fun,sch }} \rightarrow \mathcal{A}_{\mathrm{IP}, Z \text {,Fun, }}$ and we define $\operatorname{ad}_{I P, Z, F u n, s c h}(K) \subset \operatorname{pre}_{\text {IP, } Z \text {,Fun,sch }}(K)$ to be the set of functors $F$ such that the composite of $F$ with the forgetful functor is an element of $\operatorname{ad}_{\mathrm{IP}, Z, F u n}^{k}(K)$. Lemma 10.3 shows that the forgetful functor is an equivalence of categories, and then the proof of Proposition 10.9 shows that $\operatorname{ad}_{\mathrm{IP}, Z, \mathrm{Fun}, \mathrm{sch}}$ is an ad theory and that the forgetful functor induces an isomorphism of bordism groups $\left(\Omega_{\mathrm{IP}, Z, \text { Fun }, \text { sch }}\right)_{*} \rightarrow\left(\Omega_{\mathrm{IP}, Z, \text { Fun }}\right)_{*}$.

In order to make $\operatorname{ad}_{\mathrm{IP}, Z, F u n, \text { sch }}$ a functor of $Z$ we need a lemma. Recall Notation 10.4 .

Lemma 10.33. Let $g: Z \rightarrow W$.

(i) For each $z \in Z$ there is a canonical isomorphism

$$
\mathbb{Z}\left[\pi_{1}(W, g(z))\right] \otimes_{\mathbb{Z}\left[\pi_{1}(Z, z)\right]}\left(C_{f: X \rightarrow Z}\right)_{z} \cong\left(C_{g \circ f: X \rightarrow W}\right)_{g(z)},
$$

and for each pair $\left(z, z^{\prime}\right)$ there is a canonical isomorphism

$$
\begin{aligned}
\mathbb{Z}\left[\pi_{1}\left(W \times W,\left(g(z), g\left(z^{\prime}\right)\right)\right)\right] \otimes_{\mathbb{Z}\left[\pi_{1}\left(Z \times Z,\left(z, z^{\prime}\right)\right)\right]}\left(E_{f: X \rightarrow Z}\right)_{\left(z, z^{\prime}\right)} \\
\\
\cong\left(E_{g \circ f: X \rightarrow W}\right)_{\left(g(z), g\left(z^{\prime}\right)\right)} .
\end{aligned}
$$


(ii) There are canonical isomorphisms

$$
\operatorname{Kan}_{g_{*}} C_{f: X \rightarrow Z} \cong C_{g \circ f: X \rightarrow W}
$$

and

$$
\operatorname{Kan}_{g_{*} \otimes g_{*}} E_{f: X \rightarrow Z} \cong E_{g \circ f: X \rightarrow W}
$$

Proof. We give the proofs for $C_{f: X \rightarrow Z}$; the proofs for $E_{f: X \rightarrow Z}$ are similar.

Proof of (i). The left action of $\pi_{1}(Z, z)$ on $\tilde{Z}_{z}$ is given by

$$
\delta \cdot \epsilon=\epsilon \delta^{-1} \text {. }
$$

There is a homeomorphism

$$
\pi_{1}(W, g(z)) \times_{\pi_{1}(Z, z)} \tilde{Z}_{z} \rightarrow g^{*} \tilde{W}_{g(z)}
$$

which takes $(\alpha, \epsilon)$ to $\left(\epsilon(1),(g \circ \epsilon) \alpha^{-1}\right)$. Pulling back to $X$ gives a homeomorphism

$$
\begin{aligned}
\pi_{1}(W, g(z)) \times_{\pi_{1}(Z, z)} f^{*} \tilde{Z}_{z} \approx f^{*}\left(\pi_{1}(W, g(z)) \times_{\pi_{1}(Z, z)} \tilde{Z}_{z}\right) & \approx f^{*} g^{*} \tilde{W}_{g(z)} \\
& \approx(g \circ f)^{*} \tilde{W}_{g(z)} .
\end{aligned}
$$

Since (by Definition 10.27) the restriction of $f^{*} \tilde{Z}_{z}$ to each $U \in \mathcal{U}_{f^{*} \tilde{Z}_{z}}$ is trivial, the canonical map

$$
\mathbb{Z}\left[\pi_{1}(W, g(z))\right] \otimes_{\mathbb{Z}\left[\pi_{1}(Z, z)\right]} I S_{*}^{\bar{n}}(T ; \mathbb{Z}) \rightarrow I S_{*}^{\bar{n}}\left(\pi_{1}(W, g(z)) \times_{\pi_{1}(Z, z)} T ; \mathbb{Z}\right)
$$

is an isomorphism for each $T \in \mathcal{U}_{f^{*} \tilde{Z}_{z}}$. Now the following composite (where the first and fourth isomorphisms are given by [FM13b, Proposition 6.3]) is the required isomorphism.

$$
\begin{aligned}
& \mathbb{Z}\left[\pi_{1}(W, g(z))\right] \otimes_{\mathbb{Z}\left[\pi_{1}(Z, z)\right]}\left(C_{f: X \rightarrow Z)_{z}}\right. \\
& =\mathbb{Z}\left[\pi_{1}(W, g(z))\right] \otimes_{\mathbb{Z}\left[\pi_{1}(Z, z)\right]} I S_{*}^{\bar{n}, \mathcal{U}_{f *} \tilde{z}_{z}}\left(f^{*} \tilde{Z}_{z} ; \mathbb{Z}\right) \\
& \cong \mathbb{Z}\left[\pi_{1}(W, g(z))\right] \otimes_{\mathbb{Z}\left[\pi_{1}(Z, z)\right]} \operatorname{colim} I S_{*}^{\bar{n}}(T ; \mathbb{Z}) \\
& \cong \operatorname{colim}\left(\mathbb{Z}\left[\pi_{1}(W, g(z))\right] \otimes_{\mathbb{Z}\left[\pi_{1}(Z, z)\right]} I S_{*}^{\bar{n}}(T ; \mathbb{Z})\right) \\
& \cong \operatorname{colim} I S_{*}^{\bar{n}}\left(\pi_{1}(W, g(z)) \times_{\pi_{1}(Z, z)} T ; \mathbb{Z}\right) \\
& \cong I S_{*}^{\bar{n}, \mathcal{U}_{(g \circ f) *} \tilde{W}_{g(z)}}\left((g \circ f)^{*} \tilde{W}_{g(z)} ; \mathbb{Z}\right)=\left(C_{g \circ f: X \rightarrow W}\right)_{g(z)}
\end{aligned}
$$

Proof of (ii). As $z$ varies, the following composite (where the second map is the isomorphism from part (i)) gives a map of $\mathbb{Z}\left[\pi_{1} Z\right]$ modules:

$$
\left(C_{f: X \rightarrow Z}\right)_{z} \rightarrow \mathbb{Z}\left[\pi_{1}(W, g(z))\right] \otimes_{\mathbb{Z}\left[\pi_{1}(Z, z)\right]}\left(C_{f: X \rightarrow Z}\right)_{z} \stackrel{\cong}{\rightrightarrows}\left(C_{g \circ f: X \rightarrow W}\right)_{g(z)},
$$

and this induces a map of $\mathbb{Z}\left[\pi_{1} W\right]$ modules

$$
h: \operatorname{Kan}_{g_{*}} C_{f: X \rightarrow Z} \rightarrow C_{g \circ f: X \rightarrow W} .
$$


It suffices to show that $h_{g(z)}$ is an isomorphism for any z. By Remark B.3, it suffices to show that the composite

$$
\begin{aligned}
\mathbb{Z}\left[\pi_{1}(W, g(z)] \otimes_{\mathbb{Z}\left[\pi_{1}(Z, z)\right]}\left(C_{f: X \rightarrow Z}\right)_{z} \cong\left(\operatorname{Kan}_{g_{*}}\left(C_{f: X \rightarrow Z}\right)\right)_{g(z)}\right. \\
\stackrel{h_{g(z)}}{\longrightarrow}\left(C_{g \circ f: X \rightarrow W}\right)_{g(z)}
\end{aligned}
$$

is an isomorphism. But this composite is the same as the map in part (i), since both are $\mathbb{Z}\left[\pi_{1}(W, g(z)]\right.$ linear and they agree on $\left(C_{f: X \rightarrow Z}\right)_{z}$.

Now for a map $g: Z \rightarrow W$ we define a functor

$$
g_{\text {Fun,sch }}: \mathcal{A}_{\mathrm{IP}, Z, \text { Fun,sch }} \rightarrow \mathcal{A}_{\mathrm{IP}, W, \text { Fun,sch }}
$$

by

$$
\begin{aligned}
g_{\text {Fun }, \mathrm{sch}}\left(f: X \rightarrow Z, \xi, \mathbb{C}, \lambda_{1}, \mathbb{E}, \lambda_{2}\right) & \\
& =\left(g \circ f: X \rightarrow W, \xi, g_{\mathrm{sch}} \mathbb{C}, \lambda_{1}^{\prime},(g \times g)_{\mathrm{sch}} \mathbb{E}, \lambda_{2}^{\prime}\right),
\end{aligned}
$$

where $\lambda_{1}^{\prime}$ is the composite

$$
\begin{aligned}
\mathbb{Z}\left[\pi_{1} W\right]\left\langle g_{\mathrm{sch}} \mathbb{C}\right\rangle \cong \operatorname{Kan}_{g_{*}} \mathbb{Z}\left[\pi_{1} Z\right]\langle\mathbb{C}\rangle \stackrel{\operatorname{Kan}_{g_{*}} \lambda_{1}}{\longrightarrow} \operatorname{Kan}_{g_{*}} C_{f: X \rightarrow Z} \\
\cong C_{g \circ f: X \rightarrow W}
\end{aligned}
$$

(where the first isomorphism is Lemma 10.5 and the second is Lemma 10.33) and $\lambda_{2}$ is the composite

$$
\begin{aligned}
& \left(\mathbb{Z}\left[\pi_{1} W\right]^{\text {op }} \otimes \mathbb{Z}\left[\pi_{1} W\right]\right)\left\langle(g \times g)_{\text {sch }} \mathbb{E}\right\rangle \\
& \cong \operatorname{Kan}_{g_{*} \otimes g_{*}}\left(\mathbb{Z}\left[\pi_{1} Z\right]^{\text {op }} \otimes \mathbb{Z}\left[\pi_{1} Z\right]\right)\langle\mathbb{E}\rangle \\
& \stackrel{\operatorname{Kan}_{g_{*} \otimes g_{*} \lambda_{2}}}{\longrightarrow} \operatorname{Kan}_{g_{*} \otimes g_{*}} E_{f: X \rightarrow Z} \cong E_{g \circ f: X \rightarrow W}
\end{aligned}
$$

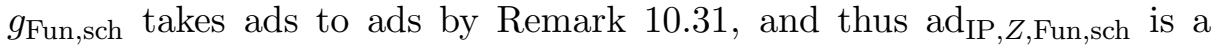
functor of $Z$ as required.

We now define

$$
\Phi^{\prime}(Z)=\mathrm{Q}_{\mathrm{IP}, Z, \text { Fun,sch }}
$$

10.11. Symmetric signature. First we give the analog of a definition from Section 8 .

Definition 10.34. For an object $f: X \rightarrow Z$ of $\mathcal{A}_{\mathrm{IP}, Z}$, define $A_{f: X \rightarrow Z}$ to be the chain complex of left $\mathbb{Z}\left[\pi_{1} Z\right]$ modules with

$$
\left(A_{f: X \rightarrow Z}\right)_{z}=I S_{*}^{\overline{0}, \mathcal{U}_{f *}^{*} Z_{z}}\left(f^{*} Z_{z} ; \mathbb{Z}\right) .
$$

For each $z \in Z$, the canonical map

$$
\mathbb{Z} \otimes_{\mathbb{Z}\left[\pi_{1}(Z, z)\right]}\left(A_{f: X \rightarrow Z)_{z}}=\mathbb{Z} \otimes_{\mathbb{Z}\left[\pi_{1}(Z, z)\right]} I S_{*}^{\overline{0}, \mathcal{U}_{f^{*} Z_{z}}}\left(f^{*} Z_{z} ; \mathbb{Z}\right) \rightarrow I S_{*}^{\overline{0}, \mathcal{U}_{X}}(X ; \mathbb{Z})\right.
$$


is an isomorphism, because it is the same as the following composite (where the first and fourth isomorphisms are given by [FM13b, Proposition 6.3]):

$$
\begin{aligned}
& \mathbb{Z} \otimes_{\mathbb{Z}\left[\pi_{1}(Z, z)\right]} I S_{*}^{\overline{0}, \mathcal{U}_{f^{*} Z_{z}}}\left(f^{*} Z_{z} ; \mathbb{Z}\right) \cong \mathbb{Z} \otimes_{\mathbb{Z}\left[\pi_{1}(Z, z)\right]} \operatorname{colim} I S_{*}^{\overline{0}}(V ; \mathbb{Z}) \\
& \quad \cong \operatorname{colim}\left(\mathbb{Z} \otimes_{\mathbb{Z}\left[\pi_{1}(Z, z)\right]} I S_{*}^{\overline{0}}(V ; \mathbb{Z})\right) \cong \operatorname{colim} I S_{*}^{\overline{0}}(U ; \mathbb{Z}) \cong I S_{*}^{\overline{0}, \mathcal{U}_{X}}(X ; \mathbb{Z}) .
\end{aligned}
$$

Recall that we write $\mathcal{Z}$ for the constant $\mathbb{Z}\left[\pi_{1} Z\right]$ module with value $\mathbb{Z}$. Using Remark 7.1, we obtain an isomorphism

$$
\mathcal{Z} \otimes_{\mathbb{Z}\left[\pi_{1} Z\right]} A_{f: X \rightarrow Z} \cong \mathbb{Z} \otimes_{\mathbb{Z}\left[\pi_{1}(Z, z)\right]}\left(A_{f: X \rightarrow Z)_{z}} \cong I S_{*}^{\overline{0}, \mathcal{U}_{X}}(X ; \mathbb{Z})\right.
$$

which the reader can check is independent of the choice of $z$.

Definition 10.35. Define a functor

$$
\operatorname{sig}: \mathcal{A}_{\mathrm{IP}, Z, \text { Fun,sch }} \rightarrow \mathcal{A}_{\mathrm{Rel}, \mathrm{sch}}^{\mathbb{Z}\left[\pi_{1} Z\right]}
$$

by

$$
\operatorname{sig}\left(f: X \rightarrow Z, \xi, \mathbb{C}, \lambda_{1}, \mathbb{E}, \lambda_{2}\right)=(\mathbb{C}, \mathbb{E}, \gamma, \phi),
$$

where $\gamma$ is the composite

$$
\begin{aligned}
\left(\mathbb{Z}\left[\pi_{1} Z\right]\langle\mathbb{C}\rangle\right)^{t} \otimes \mathbb{Z}\left[\pi_{1} Z\right]\langle\mathbb{C}\rangle \stackrel{\lambda_{1} \otimes \lambda_{1}}{\longrightarrow}\left(C_{f: X \rightarrow Z}\right)^{t} \otimes C_{f: X \rightarrow Z} \stackrel{\times}{\rightarrow} E_{f: X \rightarrow Z} \\
\stackrel{\lambda_{2}^{-1}}{\longrightarrow}\left(\mathbb{Z}\left[\pi_{1} Z\right]^{\text {op }} \otimes \mathbb{Z}\left[\pi_{1} Z\right]\right)\langle\mathbb{E}\rangle
\end{aligned}
$$

and $\phi$ is the image of $\xi$ under the following composite (where the arrow is induced by the diagonal map):

$$
\begin{aligned}
I S_{*}^{\overline{0}, \mathcal{U}_{X}}(X ; \mathbb{Z}) \cong \mathcal{Z} \otimes_{\mathbb{Z}\left[\pi_{1} Z\right]} A_{f: X \rightarrow Z} \rightarrow \mathcal{Z} \otimes_{\mathbb{Z}\left[\pi_{1} Z\right]} E_{f: X \rightarrow Z} \\
\stackrel{1 \otimes \lambda_{2}^{-1}}{\longrightarrow} \mathcal{Z} \otimes_{\mathbb{Z}\left[\pi_{1} Z\right]}\left(\mathbb{Z}\left[\pi_{1} Z\right]^{\text {op }} \otimes \mathbb{Z}\left[\pi_{1} Z\right]\right)\langle\mathbb{E}\rangle
\end{aligned}
$$

The definitions show that sig is a natural transformation as $Z$ varies.

Proposition 10.36. If $K$ is strict and $F \in \operatorname{ad}_{\mathrm{IP}, Z, F u n, s c h}^{k}(K)$ then $\operatorname{sig} \circ F \in$ $\left(\operatorname{ad}_{\mathrm{Rel}, \mathrm{sch}, \geq 0}^{\mathbb{Z}\left[\pi_{1} Z\right]}\right)^{k}(K)$.

Proof. Let $F \in \operatorname{ad}_{\mathrm{IP}, Z \text {,Fun,sch }}(K)$. By Definition 10.20 we need to show that $\Theta_{\text {Rel }} \circ$ sig $\circ F$ is an ad, and for this we need to show (see Remark 10.16) that $\Theta_{\text {Rel }} \circ \operatorname{sig} \circ F$ is closed and well-behaved and that its composite with the map

$$
\Lambda: \mathcal{A}_{\mathrm{Rel}}^{\mathbb{Z}\left[\pi_{1} Z\right]} \rightarrow \mathcal{A}_{\mathrm{rel}}^{\mathbb{Z}\left[\pi_{1} Z\right]}
$$

satisfies the analog of [LM, part (i)(b) of Definition 12.10].

$\Theta_{\text {Rel }} \circ \operatorname{sig} \circ F$ is closed by property (c) of Definition 10.28.

For the proof that $\Theta_{\text {Rel }} \circ$ sigo $F$ is well-behaved, first note that by Definition 10.28(a), $F$ is balanced, so we can write

$$
F(\sigma, o)=\left(f_{\sigma}: X_{\sigma, o} \rightarrow Z, \xi_{\sigma, o}, \mathbb{C}_{\sigma},\left(\lambda_{1}\right)_{\sigma}, \mathbb{E}_{\sigma},\left(\lambda_{2}\right)_{\sigma}\right) .
$$

Also write $Y_{\sigma}$ for the underlying $\partial$-IP-space of $X_{\sigma, o}$ (forgetting the orientation). It suffices to show that the functors $C_{f_{\sigma}: Y_{\sigma} \rightarrow Z}$ and $E_{f_{\sigma}: Y_{\sigma} \rightarrow Z}$ are 
well-behaved. We will prove this for the first, the proof for the second is similar.

Choose $z \in Z$. It suffices to show that the functor $I S_{i}^{\bar{n}, \mathcal{U}_{f_{\sigma}^{*}\left(\tilde{Z}_{z}\right)}}\left(f_{\sigma}^{*}\left(\tilde{Z}_{z}\right) ; \mathbb{Z}\right)$ is well-behaved. Let $\sigma \in K$, and for each $\tau \subset \sigma$ let $\mathcal{W}_{\tau}$ be the collection of all intersections of sets in $\mathcal{U}_{Y_{\tau}}$, and for each $U \in \mathcal{W}_{\tau}$ let $\tilde{U}$ be the inverse image of $U$ in $f_{\tau}^{*}\left(\tilde{Z}_{z}\right)$. The map

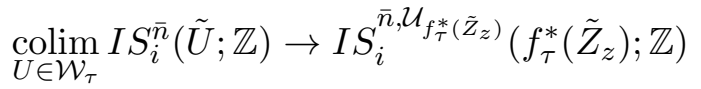

is an isomorphism for each $\tau$ by [FM13b, Proposition 6.3], so the wellbehavedness will be an immediate consequence of Lemma D.1(iii), with $\mathcal{S}=$ $\cup_{\tau \subset \sigma} \mathcal{W}_{\tau}$ and $\mathcal{S}^{\prime}=\cup_{\tau \in \partial \sigma} \mathcal{W}_{\tau}$, once we verify the hypotheses of that Lemma. Hypothesis (a) follows from Definition 10.27, and hypothesis (b) is part (iii) of Lemma 10.37 below. For hypothesis (c), it suffices to show that for each $U \in \mathcal{S}$ the map

$$
I S_{i}^{\bar{n}}(U ; \mathbb{Z}) / \underset{V \in \mathcal{S} \text { with } V \subsetneq U}{\operatorname{colim}} I S_{i}^{\bar{n}}(V ; \mathbb{Z}) \rightarrow S_{i}(U ; \mathbb{Z}) / \operatorname{V\in \mathcal {S}}_{\operatorname{colith} V \subsetneq U} S_{i}(V ; \mathbb{Z})
$$

is a monomorphism (because the target of this map is free over $\mathbb{Z}$ ) and this follows from parts (iii) and (ii) of Lemma 10.37. This concludes the proof that sig $\circ F$ is well-behaved.

To see that $\Lambda \circ \Theta_{\text {Rel }} \circ$ sig $\circ F$ satisfies the analog of [LM, part (i)(b) of Definition 12.10], we first observe that the map

$$
I S_{*}^{\bar{n}, \mathcal{U}_{f_{\sigma}^{*}\left(\tilde{Z}_{z}\right)}}\left(f_{\sigma}^{*}\left(\tilde{Z}_{z}\right) ; \mathbb{Z}\right) \rightarrow I S_{*}^{\bar{n}}\left(f_{\sigma}^{*}\left(\tilde{Z}_{z}\right) ; \mathbb{Z}\right)
$$

is a quasi-isomorphism by Remark 10.26, and that, if $\mathcal{S}^{\prime}$ is as above, the composite

$$
\operatorname{colim}_{\tau \subset \partial \sigma} I S_{*}^{\bar{n}, \mathcal{U} f_{\tau}^{*}\left(\tilde{Z}_{z}\right)}\left(f_{\tau}^{*}\left(\tilde{Z}_{z}\right) ; \mathbb{Z}\right) \cong \operatorname{colim}_{U \in \mathcal{S}^{\prime}} I S_{*}^{\bar{n}}(\tilde{U} ; \mathbb{Z}) \rightarrow I S_{*}^{\bar{n}}\left(\partial f_{\sigma}^{*}\left(\tilde{Z}_{z}\right) ; \mathbb{Z}\right)
$$

is a quasi-isomorphism by Lemma 10.37(iv). It therefore suffices to show that the map

$$
\begin{aligned}
& H_{*}\left(\operatorname{Hom}_{\mathbb{Z}\left[\pi_{1}(Z, z)\right]}\left(I S_{*}^{\bar{n}}\left(f_{\sigma}^{*}\left(\tilde{Z}_{z}\right) ; \mathbb{Z}\right), \mathbb{Z}\left[\pi_{1}(Z, z)\right]\right)\right. \\
& \stackrel{\backslash\left(\left(1 \otimes \gamma_{\sigma}\right)_{*}\right)^{-1}\left(\left[\phi_{\sigma}\right]\right)}{\longrightarrow} I H_{\operatorname{dim} \sigma-k-*}^{\bar{n}}\left(f_{\sigma}^{*}\left(\tilde{Z}_{z}\right), \partial f_{\sigma}^{*}\left(\tilde{Z}_{z}\right) ; \mathbb{Z}\right)
\end{aligned}
$$

is an isomorphism for each $\sigma$. The rest of the proof is the same as the corresponding part of the proof of Proposition [6.6. except that $Y_{\sigma}$ is replaced by $f_{\sigma}^{*}\left(\tilde{Z}_{z}\right)$.

With notation as in the proof of well-behavedness in Proposition 10.36, say that a subset $\mathcal{T}$ of $\mathcal{S}$ is saturated if for every $U \in \mathcal{T}$ we have $\{V \mid V \in$ $\mathcal{S}$ and $V \subset U\} \subset \mathcal{T}$. For every saturated $\mathcal{T}$, write $X_{\mathcal{T}}$ for $\operatorname{colim}_{U \in \mathcal{T}} U$, and give this the filtration which restricts to the filtration of Proposition A.2 on each $U \in \mathcal{T}$ (note that an open subset of a $\partial$-pseudomanifold is a $\partial$-pseudomanifold by [F, Lemma 2.7.7]). Write $\tilde{X}_{\mathcal{T}}$ for $\operatorname{colim}_{U \in \mathcal{T}} \tilde{U}$. 
Lemma 10.37. (i) For every pair of saturated sets $\mathcal{T}^{\prime} \subset \mathcal{T}$, there is a neighborhood $W$ of $X_{\mathcal{T}^{\prime}}$ in $X_{\mathcal{T}}$ such that the inclusion $X_{\mathcal{T}} \rightarrow W$ is a stratified deformation retract, where the retraction $r: W \rightarrow X_{\mathcal{T}}$, has the property that $r(x) \in U$ whenever $x \in U \in \mathcal{T}$.

(ii) For every saturated $\mathcal{T}$ and every $i \in \mathbb{Z}$, the intersection of $I S_{i}^{\bar{n}}\left(X_{K} ; \mathbb{Z}\right)$ and $\sum_{U \in \mathcal{T}} S_{i}(U ; \mathbb{Z})$ (considered as subgroups of $S_{i}\left(X_{K} ; \mathbb{Z}\right)$ ) is $\sum_{U \in \mathcal{T}} I S_{i}^{\bar{n}}(U)$.

(iii) If $K$ is strict then for every saturated $\mathcal{T}$ the map

$$
\underset{U \in \mathcal{T}}{\operatorname{colim}} I S_{*}^{\bar{n}}(U ; \mathbb{Z}) \rightarrow \sum_{U \in \mathcal{T}} I S_{*}^{\bar{n}}(U ; \mathbb{Z})
$$

is an isomorphism.

(iv) For every saturated $\mathcal{T}$ the map

$$
\underset{U \in \mathcal{T}}{\operatorname{colim}} I S_{*}^{\bar{n}}(\tilde{U} ; \mathbb{Z}) \rightarrow I S_{*}^{\bar{n}}\left(\tilde{X}_{\mathcal{T}}\right)
$$

is a quasi-isomorphism.

The proof is the same as the proof of Lemma 6.7.

10.12. Proof of Lemma 10.13. We need to show that $\alpha^{-1}[X]_{\mathrm{IP}}$ is taken to $[X]_{\mathbb{L}}$ by the composite

$$
\begin{aligned}
X_{+} \wedge \mathbf{Q}_{\mathrm{IP}} \stackrel{\simeq}{\longleftarrow} X_{+} \wedge \mathbf{Q}_{\mathrm{IP}, * \text { Fun }, \mathrm{sch}} \stackrel{1 \wedge \text { sig }}{\longrightarrow} X_{+} & \wedge \mathbf{Q}_{\text {Rel }, \mathrm{sch}, \geq 0}^{\mathbb{Z}} \stackrel{\simeq}{\longleftarrow} X_{+} \wedge \mathbf{Q}_{\mathrm{fin}, \mathrm{sch}, \geq 0}^{\mathbb{Z}} \\
& \rightarrow X_{+} \wedge \mathbf{Q}_{\mathrm{sch}, \geq 0}^{\mathbb{Z}} \rightarrow X_{+} \wedge \mathbf{Q}_{\geq 0}^{\mathbb{Z}} .
\end{aligned}
$$

This is the same as the composite

$$
\begin{aligned}
X_{+} \wedge \mathbf{Q}_{\mathrm{IP}} \stackrel{\simeq}{\longleftarrow} X_{+} \wedge \mathbf{Q}_{\mathrm{IP}, * \text { Fun }, \mathrm{sch}} \stackrel{1 \wedge \text { sig }}{\longrightarrow} X_{+} \wedge \mathbf{Q}_{\text {Rel }, \mathrm{sch}, \geq 0}^{\mathbb{Z}} \rightarrow X_{+} \wedge \mathbf{Q}_{\mathrm{Rel}, \geq 0}^{\mathbb{Z}} \\
\stackrel{\simeq}{\longleftarrow} X_{+} \wedge \mathbf{Q}_{\mathrm{fin}, \geq 0}^{\mathbb{Z}} \rightarrow X_{+} \wedge \mathbf{Q}_{\geq 0}^{\mathbb{Z}},
\end{aligned}
$$

which by Remark 10.22 is homotopic to the composite

$$
\begin{aligned}
X_{+} \wedge \mathbf{Q}_{\mathrm{IP}} \stackrel{\simeq}{\longleftarrow} X_{+} \wedge \mathbf{Q}_{\mathrm{IP}, * \text { Fun }, \mathrm{sch}} \stackrel{1 \wedge \mathrm{sig}}{\longrightarrow} & X_{+} \wedge \mathbf{Q}_{\mathrm{Rel}, \mathrm{sch}, \geq 0}^{\mathbb{Z}} \rightarrow X_{+} \wedge \mathbf{Q}_{\mathrm{Rel}, \geq 0}^{\mathbb{Z}} \\
& \stackrel{1 \wedge \Lambda}{\longrightarrow} X_{+} \wedge \mathbf{Q}_{\mathrm{rel}, \geq 0}^{\mathbb{Z}} \stackrel{\simeq}{\longleftarrow} X_{+} \wedge \mathbf{Q}_{\geq 0}^{\mathbb{Z}} .
\end{aligned}
$$

Now Lemma 10.13 follows from Definition 9.6(ii) and

Lemma 10.38. The diagram

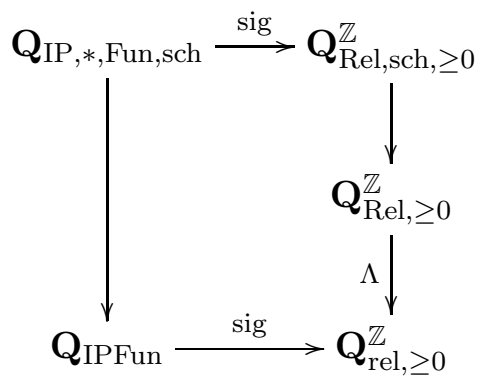


commutes up to homotopy, where the upper map was constructed in Subsection 10.11, the lower map in Subsection 6.3, and the left map is induced by the forgetful functor.

Proof. By the analog of [LM, Proposition 14.5], it suffices to give a natural quasi-isomorphism $\nu$ from the composite

$$
\mathcal{A}_{\mathrm{IP}, * \text { Fun }, \text { sch }} \stackrel{\text { sig }}{\rightarrow} \mathcal{A}_{\mathrm{Rel}, \mathrm{sch}}^{\mathbb{Z}} \rightarrow \mathcal{A}_{\mathrm{Rel}}^{\mathbb{Z}} \stackrel{\Lambda}{\rightarrow} \mathcal{A}_{\mathrm{rel}}^{\mathbb{Z}}
$$

to the composite

$$
\mathcal{A}_{\mathrm{IP}, * \text { Fun }, \text { sch }} \rightarrow \mathcal{A}_{\mathrm{IPFun}} \stackrel{\text { sig }}{\rightarrow} \mathcal{A}_{\mathrm{rel}}^{\mathbb{Z}} .
$$

By Definition 10.32, $C_{f: X \rightarrow *}=I S_{*}^{\bar{n}, \mathcal{U}_{X}}(X ; \mathbb{Z})$ and $E_{f: X \rightarrow *}=I S_{*}^{Q_{\bar{n}}, \bar{n}}, \mathcal{U}_{X \times X}(X \times$ $X ; \mathbb{Z})$, and by Definition 10.34 we have $A_{f: X \rightarrow *}=I S_{*}^{\overline{0}, \mathcal{U}_{X}}(X ; \mathbb{Z})$. Thus the first composite takes the object

$$
\left(X \rightarrow *, \xi, \mathbb{C}, \lambda_{1}, \mathbb{E}, \lambda_{2}\right)
$$

to

where $\beta$ is the map

$$
(\mathbb{Z}\langle\mathbb{C}\rangle, \mathbb{Z}\langle\mathbb{E}\rangle, \beta, \varphi)
$$

$$
\begin{aligned}
& (\mathbb{Z}\langle\mathbb{C}\rangle)^{t} \otimes \mathbb{Z}\langle\mathbb{C}\rangle \stackrel{\lambda_{1} \otimes \lambda_{1}}{\longrightarrow}\left(I S_{*}^{\bar{n}, \mathcal{U}_{X}}(X ; \mathbb{Z})\right)^{t} \otimes I S_{*}^{\bar{n}, \mathcal{U}_{X}}(X ; \mathbb{Z}) \stackrel{\times}{\longrightarrow} I S_{*}^{Q_{\bar{n}, \bar{n}}, \mathcal{U}_{X \times X}}(X \times X ; \mathbb{Z}) \\
& \stackrel{\lambda_{2}^{-1}}{\longrightarrow} \mathbb{Z}\langle\mathbb{E}\rangle
\end{aligned}
$$

and $\varphi$ is the image of $\xi$ under the diagonal map

$$
I S_{*}^{\overline{0}, \mathcal{U}_{X}}(X ; \mathbb{Z}) \rightarrow I S_{*}^{Q_{\bar{n}}, \bar{n}}, \mathcal{U}_{X \times X}(X \times X ; \mathbb{Z}) .
$$

The second composite takes $\left(X \rightarrow *, \xi, \mathbb{C}, \lambda_{1}, \mathbb{E}, \lambda_{2}\right)$ to

$$
\left(I S_{*}^{\bar{n}}(X ; \mathbb{Z}), I S_{*}^{Q_{\bar{n}}, \bar{n}}(X \times X ; \mathbb{Z}), \beta^{\prime}, \varphi^{\prime}\right),
$$

where $\beta^{\prime}$ is the map

$$
\left(I S_{*}^{\bar{n}}(X ; \mathbb{Z})\right)^{t} \otimes I S_{*}^{\bar{n}}(X ; \mathbb{Z}) \stackrel{\times}{\rightarrow} I S_{*}^{Q \bar{n}, \bar{n}}(X \times X ; \mathbb{Z})
$$

and $\varphi^{\prime}$ is the image of $\xi$ under the map

$$
I S_{*}^{\overline{0}, \mathcal{U}_{X}}(X ; \mathbb{Z}) \rightarrow I S_{*}^{\overline{0}}(X ; \mathbb{Z}) \rightarrow I S_{*}^{Q_{\bar{n}, \bar{n}}}(X \times X ; \mathbb{Z}) .
$$

The natural quasi-isomorphism is given by the maps

$$
\mathbb{Z}\langle\mathbb{C}\rangle \stackrel{\lambda_{1}}{\longrightarrow} I S_{*}^{\bar{n}, \mathcal{U}_{X}}(X ; \mathbb{Z}) \rightarrow I S_{*}^{\bar{n}}(X ; \mathbb{Z})
$$

and

$$
\mathbb{Z}\langle\mathbb{E}\rangle \stackrel{\lambda_{2}}{\longrightarrow} I S_{*}^{Q_{\bar{n}}, \bar{n}}, \mathcal{U}_{X \times X}(X \times X ; \mathbb{Z}) \rightarrow I S_{*}^{Q_{\bar{n}, \bar{n}}}(X \times X ; \mathbb{Z})
$$


10.13. Proof of Lemma 10.14. Let $X$ be a compact connected oriented IP-space of dimension $n$. Recall Definition [9.6, and let $[X \stackrel{\text { id }}{\rightarrow} X]$ denote the class of the identity map in $\left(\Omega_{\mathrm{IP}}\right)_{n}(X)$. We need to show that the image of $[X \stackrel{\text { id }}{\rightarrow} X]$ under the following composite is $\sigma_{\mathrm{IP}}^{*}(X)$.

$$
\begin{aligned}
& \left(\Omega_{\mathrm{IP}, X}\right)_{n} \stackrel{\simeq}{\longleftarrow}\left(\Omega_{\mathrm{IP}, X, \mathrm{Fun}, \mathrm{sch}}\right)_{n} \stackrel{\text { sig }}{\longrightarrow}\left(\Omega_{\mathrm{Rel}, \mathrm{sch}, \geq 0}^{\mathbb{Z}\left[\pi_{1} X\right]}\right)_{n} \stackrel{\simeq}{\stackrel{(}{\longleftarrow}}\left(\Omega_{\mathrm{fin}, \mathrm{sch}, \geq 0}^{\mathbb{Z}\left[\pi_{1} X\right]}\right)_{n} \rightarrow\left(\Omega_{\mathrm{sch}, \geq 0}^{\mathbb{Z}\left[\pi_{1} X\right]}\right)_{n} \\
& \rightarrow\left(\Omega_{\geq 0}^{\mathbb{Z}\left[\pi_{1} X\right]}\right)_{n}=L^{n}\left(\mathbb{Z}\left[\pi_{1} X\right]\right)
\end{aligned}
$$

Using Remark 10.22 we see that this is the same as the composite

$$
\begin{array}{r}
\left(\Omega_{\mathrm{IP}, X}\right)_{n} \stackrel{\simeq}{\longleftarrow}\left(\Omega_{\mathrm{IP}, X, \text { Fun,sch }}\right)_{n} \stackrel{\text { sig }}{\longrightarrow}\left(\Omega_{\mathrm{Rel}, \mathrm{sch}, \geq 0}^{\mathbb{Z}\left[\pi_{1} X\right]}\right)_{n} \rightarrow\left(\Omega_{\mathrm{Rel}, \geq 0}^{\mathbb{Z}\left[\pi_{1} X\right]}\right)_{n} \stackrel{\Lambda}{\rightarrow}\left(\Omega_{\mathrm{rel}, \geq 0}^{\mathbb{Z}\left[\pi_{1} X\right]}\right)_{n} \\
\stackrel{\cong}{\longleftarrow}\left(\Omega_{\geq 0}^{\mathbb{Z}\left[\pi_{1} X\right]}\right)_{n}=L^{n}\left(\mathbb{Z}\left[\pi_{1} X\right]\right)
\end{array}
$$

Recall that the first map in this composite is induced by the forgetful functor, so the image of $[X \stackrel{\text { id }}{\rightarrow} X]$ under this map is the class of any object of the form

$$
\left(\text { id }: X \rightarrow X, \xi, \mathbb{C}, \lambda_{1}, \mathbb{E}, \lambda_{2}\right) \text {. }
$$

By Definition 8.4 it suffices to show

Lemma 10.39. The image of the class of (id : $\left.X \rightarrow X, \xi, \mathbb{C}, \lambda_{1}, \mathbb{E}, \lambda_{2}\right)$ under the composite

$$
\left(\Omega_{\mathrm{IP}, X, \text { Fun }, \mathrm{sch}}\right)_{n} \stackrel{\mathrm{sig}}{\rightarrow}\left(\Omega_{\mathrm{Rel}, \mathrm{sch}, \geq 0}^{\mathbb{Z}\left[\pi_{1} X\right]}\right)_{n} \rightarrow\left(\Omega_{\mathrm{Rel}, \geq 0}^{\mathbb{Z}\left[\pi_{1} X\right]}\right)_{n} \stackrel{\Lambda}{\rightarrow}\left(\Omega_{\mathrm{rel}, \geq 0}^{\mathbb{Z}\left[\pi_{1} X\right]}\right)_{n}
$$

is the class of the object $\left(C_{X}, D_{X}, \beta_{X}, \varphi\right)$ given by Definition 8.2.

Proof. The image of (id : $X \rightarrow X, \xi, \mathbb{C}, \lambda_{1}, \mathbb{E}, \lambda_{2}$ ) under the composite is represented by the following $*$-ad (where $\beta$ is given by Definition 10.35 and Remark 10.16, and $\phi$ is given by Definition 10.35)

$$
F=\left(\mathbb{Z}\left[\pi_{1} X\right]\langle\mathbb{C}\rangle, \mathcal{Z} \otimes_{\mathbb{Z}\left[\pi_{1} X\right]}\left(\mathbb{Z}\left[\pi_{1} X\right]^{\text {op }} \otimes \mathbb{Z}\left[\pi_{1} X\right]\right)\langle\mathbb{E}\rangle, \beta, \phi\right) .
$$

The following maps give a morphism of ads from this to $\left(C_{X}, D_{X}, \beta_{X}, \varphi\right)$ :

$$
\mathbb{Z}\left[\pi_{1} X\right]\langle\mathbb{C}\rangle \stackrel{\lambda_{1}}{\rightarrow} C_{\mathrm{id}: X \rightarrow X} \rightarrow C_{X}
$$

and

$$
\begin{aligned}
\mathcal{Z} \otimes_{\mathbb{Z}\left[\pi_{1} X\right]}\left(\mathbb{Z}\left[\pi_{1} X\right]^{\text {op }} \otimes \mathbb{Z}\left[\pi_{1} X\right]\right)\langle\mathbb{E}\rangle \stackrel{1 \otimes \lambda_{2}}{\longrightarrow} \mathcal{Z} \otimes_{\mathbb{Z}\left[\pi_{1} X\right]} E_{\mathrm{id}: X \rightarrow X} & \rightarrow \mathcal{Z} \otimes_{\mathbb{Z}\left[\pi_{1} X\right]} E_{X} .
\end{aligned}
$$

The result now follows from [LM, Lemma 13.5]. 


\section{The Stratified Novikov Conjecture}

Let $G$ be a discrete group and $B G$ its classifying space. Recall that the strong Novikov conjecture for $G$ asserts that the assembly map

$$
\alpha: \mathrm{L}_{n}^{\bullet}(B G) \longrightarrow L^{n}(\mathbb{Z}[G])
$$

is rationally injective. The $\mathbb{Q}$-localization of any spectrum is a graded Eilenberg-MacLane spectrum, see e.g. [Rud98, Theorem 7.11]. Thus there is a splitting

$$
\mathbb{L} \cdot(\mathbb{Z}) \otimes \mathbb{Q} \simeq \prod_{j \geq 0} K(\mathbb{Q}, 4 j) .
$$

A morphism $\phi: E \rightarrow F$ between two ring $\mathbb{Q}$-local spectra $E, F$ is a ring morphism if and only if $\phi_{*}: \pi_{*}(E) \rightarrow \pi_{*}(F)$ is a ring homomorphism (loc. cit.). The product on $\pi_{*}\left(\mathbb{L}^{\bullet}\right)$ is

$$
L^{p}(\mathbb{Z}) \otimes L^{q}(\mathbb{Z}) \longrightarrow L^{p+q}(\mathbb{Z}),
$$

given by sending chain complexes $C, D$ to their tensor product $C \otimes D$. If $p$ and $q$ are divisible by 4 (which is the only nontrivial case after tensoring with $\mathbb{Q})$, then $L^{p}(\mathbb{Z}) \cong \mathbb{Z}$, a generator is given by the symmetric signature $\sigma^{*}\left(\mathbb{C} P^{p / 2}\right) \in L^{p}(\mathbb{Z})$, and the product sends $\sigma^{*}\left(\mathbb{C} P^{p / 2}\right) \otimes \sigma^{*}\left(\mathbb{C} P^{q / 2}\right)$ to $\sigma^{*}\left(\mathbb{C} P^{p / 2} \times \mathbb{C} P^{q / 2}\right)$. Under the isomorphism induced by the splitting (11.1) on $\pi_{*}$, this product induces a product on the homotopy groups of the graded Eilenberg-MacLane spectrum, which underlies a spectrum level ring structure. By the above remark, the equivalence (11.1) is then a morphism of ring spectra. It induces natural isomorphisms of homology theories

$$
S_{X}: \mathrm{L}_{n}^{\bullet}(X) \otimes \mathbb{Q} \stackrel{\cong}{\longrightarrow} \bigoplus_{j \geq 0} H_{n-4 j}(X ; \mathbb{Q}) \text {. }
$$

and cohomology theories

$$
S^{X}:(\mathrm{L} \bullet)^{n}(X) \otimes \mathbb{Q} \stackrel{\cong}{\longrightarrow} \prod_{j \geq 0} H^{n+4 j}(X ; \mathbb{Q}) .
$$

Since $S^{X}$ is induced by a morphism of ring spectra, it maps the unit $1 \in\left(\mathrm{L}^{\bullet}\right)^{0}(\mathrm{pt}) \otimes \mathbb{Q}$ to the unit $1 \in H^{0}(\mathrm{pt} ; \mathbb{Q})$ and preserves products.

An $n$-dimensional compact IP-space $X$ possesses characteristic classes $L_{j}(X) \in H_{j}(X ; \mathbb{Q})$, which are the Poincaré duals of the Hirzebruch $L$ classes when $X$ is a smooth manifold. These classes have been introduced by Goresky and MacPherson in GM80 (at least for spaces without odd codimensional strata, but the method works whenever one has a self-dual intersection chain sheaf, see Ban07]). Goresky and MacPherson adapt a method of Thom, which exploits the bordism invariance of the signatures of transverse inverse images of maps to spheres. For singular $X$, these classes need not lift to the cohomology of $X$ under capping with the (ordinary) fundamental class. We shall denote the total $L$-class by $L(X)$. An inclusion 
$Y \hookrightarrow X$ of $\mathrm{PL}$ spaces is called normally nonsingular if $Y$ possesses a regular neighborhood in $X$ which can be given the structure of a disk-block-bundle over $Y$.

Lemma 11.1. Let $X$ be an $n$-dimensional compact IP-space. The isomorphism $S_{X}$ maps the rational L-theory fundamental class $[X]_{\mathbb{L}}$ to the GoreskyMacPherson L-class $L(X)$.

Proof. Let $Y^{m}$ be a compact IP-space and $j: Y^{m} \hookrightarrow X^{n}$ a normally nonsingular inclusion with trivial normal bundle $\nu$. Let $D \nu=D^{n-m} \times Y$ be the total space of its disk bundle, $S \nu=S^{n-m-1} \times Y$ the total space of its sphere bundle. Note that $D \nu$ is a $\partial$-IP space. Let $u \in H^{n-m}(D \nu, S \nu ; \mathbb{Q})$ be the Thom class of the normal bundle. The composition

$$
\begin{aligned}
& H_{k}(X ; \mathbb{Q}) \rightarrow H_{k}(X, X-Y ; \mathbb{Q}) \stackrel{\cong}{\cong} H_{k}(D \nu, S \nu ; \mathbb{Q}) \\
& \quad \stackrel{u \cap-}{\cong} H_{k-n+m}(D \nu ; \mathbb{Q}) \stackrel{\pi_{*}}{\cong} H_{k-n+m}(Y ; \mathbb{Q}) \stackrel{(-1)^{s}}{\rightarrow} H_{k-n+m}(Y ; \mathbb{Q}),
\end{aligned}
$$

where $s=\frac{1}{2}(n-m+1)(n-m)$ and $\pi: D \nu \rightarrow Y$ is the bundle projection, defines a map

$$
j^{!}: H_{k}(X ; \mathbb{Q}) \longrightarrow H_{k-n+m}(Y ; \mathbb{Q}) .
$$

If $Z$ is any topological space and $R$ any coefficient ring, let $\epsilon_{*}: H_{0}(Z ; R) \rightarrow R$ be the augmentation map. By the Thom-Goresky-MacPherson construction, the $L$-classes are uniquely characterized by the following two properties ([Ban07, Proposition 8.2.11]):

- If $j: Y^{m} \hookrightarrow X^{n}$ is a normally nonsingular inclusion with trivial normal bundle, then

$$
L_{k-n+m}(Y)=j^{!} L_{k}(X) .
$$

- $\epsilon_{*} L_{0}(X)=\sigma(X)$, the signature of $X$.

Thus the lemma is proven if we show

(1) If $j: Y^{m} \hookrightarrow X^{n}$ is a normally nonsingular inclusion with trivial normal bundle, then

$$
\left(S_{Y}[Y]_{\mathbb{L}}\right)_{k-n+m}=j^{!}\left(S_{X}[X]_{\mathbb{L}}\right)_{k}
$$

and

(2) $\epsilon_{*}\left(S_{X}[X]_{\mathbb{L}}\right)_{0}=\sigma(X)$.

We turn to (1). Let $\nu$ be the normal bundle of $j$. We write $S^{0}$ as $S^{0}=$ $\left\{y_{-}, y_{+}\right\}$. Let $e \in H^{1}\left(D^{1}, S^{0} ; \mathbb{Q}\right)$ be the element obtained as the image of the unit $1 \in H^{0}\left(y_{+} ; \mathbb{Q}\right)$ under the composition

$$
H^{0}\left(y_{+} ; \mathbb{Q}\right) \stackrel{\cong}{\longleftarrow} H^{0}\left(S^{0}, y_{-} ; \mathbb{Q}\right) \stackrel{\delta}{\longrightarrow} H^{1}\left(D^{1}, S^{0} ; \mathbb{Q}\right),
$$

where the left arrow is an excision isomorphism and $\delta$ is the connecting homomorphism of the triple $\left(D^{1}, S^{0}, y_{-}\right)$. The $(n-m)$-fold cross-product $e \times \cdots \times e$ yields an element $e^{n-m} \in H^{n-m}\left(D^{n-m}, S^{n-m-1} ; \mathbb{Q}\right)$. The Thom class $u$ arising in the definition of the map $j^{!}$is then given by $u=e^{n-m} \times 1 \epsilon$ $H^{n-m}(D \nu, S \nu ; \mathbb{Q})$, where $1 \in H^{0}(Y ; \mathbb{Q})$. Analogous classes in $\mathbb{L} \bullet$-homology 
and $\Omega_{\mathrm{IP}}$ can be constructed in a similar fashion: If $A$ is any abelian group, we shall briefly write $A_{\mathbb{Q}}$ for $A \otimes_{\mathbb{Z}} \mathbb{Q}$. Let $e_{\mathbb{L}} \in\left(\mathrm{L}^{\bullet}\right)^{1}\left(D^{1}, S^{0}\right)_{\mathbb{Q}}$ be the element obtained as the image of the unit $1 \in\left(\mathrm{L}^{\bullet}\right)^{0}\left(y_{+}\right)_{\mathbb{Q}}$ under the composition

$$
\left(\mathrm{L}^{\bullet}\right)^{0}\left(y_{+}\right)_{\mathbb{Q}} \cong\left(\mathrm{L}^{\bullet}\right)^{0}\left(S^{0}, y_{-}\right)_{\mathbb{Q}} \stackrel{\delta}{\longrightarrow}\left(\mathrm{L}^{\bullet}\right)^{1}\left(D^{1}, S^{0}\right)_{\mathbb{Q}}
$$

The $(n-m)$-fold cross-product $e_{\mathbb{L}} \times \cdots \times e_{\mathbb{L}}$ yields an element $e_{\mathbb{L}}^{n-m} \in$ $\left(\mathrm{L}^{\bullet}\right)^{n-m}\left(D^{n-m}, S^{n-m-1}\right)_{\mathbb{Q}}$. Set $u_{\mathbb{L}}=e_{\mathbb{L}}^{n-m} \times 1 \in\left(\mathrm{L}^{\bullet}\right)^{n-m}(D \nu, S \nu)_{\mathbb{Q}}$, where $1 \in\left(\mathrm{L}^{\bullet}\right)^{0}(Y)_{\mathbb{Q}}$ is the unit.

We claim that

$$
S^{\left(D^{1}, S^{0}\right)}\left(e_{\mathbb{L}}\right)=e .
$$

To see this, we observe that as $S$ is a natural transformation of cohomology theories, the diagram

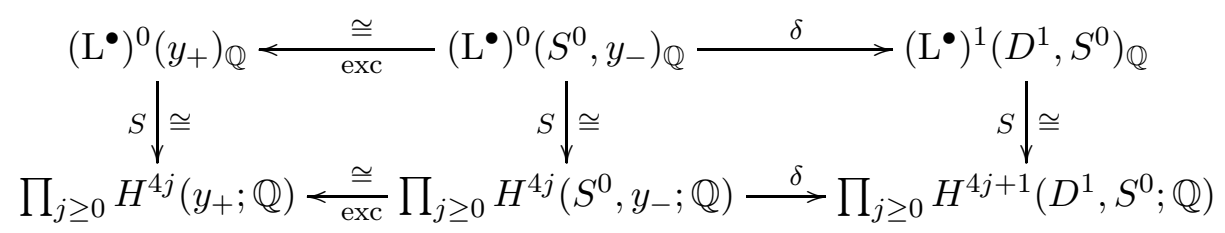

commutes and hence

$$
S\left(e_{\mathbb{L}}\right)=S \delta \operatorname{exc}^{-1}(1)=\delta \operatorname{exc}^{-1} S(1)=\delta \operatorname{exc}^{-1}(1)=e .
$$

We show next that

$$
S^{(D \nu, S \nu)}\left(u_{\mathbb{L}}\right)=u
$$

Since $S^{(D \nu, S \nu)}$ is induced by a morphism of ring spectra, it preserves products on cohomology. Thus, using (11.2),

$$
\begin{aligned}
S\left(u_{\mathbb{L}}\right) & =S\left(e_{\mathbb{L}} \times \cdots \times e_{\mathbb{L}} \times 1\right) \\
& =S\left(e_{\mathbb{L}}\right) \times \cdots \times S\left(e_{\mathbb{L}}\right) \times S(1) \\
& =e \times \cdots \times e \times 1 \\
& =u .
\end{aligned}
$$

Let $e_{\mathrm{IP}} \in\left(\Omega_{\mathrm{IP}}\right)^{1}\left(D^{1}, S^{0}\right)_{\mathbb{Q}}$ be the element obtained as the image of the unit $1 \in\left(\Omega_{\mathrm{IP}}\right)^{0}\left(y_{+}\right)_{\mathbb{Q}}$ under the composition

$$
\left(\Omega_{\mathrm{IP}}\right)^{0}\left(y_{+}\right)_{\mathbb{Q}} \stackrel{\cong}{\cong}\left(\Omega_{\mathrm{IP}}\right)^{0}\left(S^{0}, y_{-}\right)_{\mathbb{Q}} \stackrel{\delta}{\longrightarrow}\left(\Omega_{\mathrm{IP}}\right)^{1}\left(D^{1}, S^{0}\right)_{\mathbb{Q}} .
$$

The $(n-m)$-fold cross-product $e_{\mathrm{IP}} \times \cdots \times e_{\mathrm{IP}}$ yields an element $e_{\mathrm{IP}}^{n-m} \in$ $\left(\Omega_{\mathrm{IP}}\right)^{n-m}\left(D^{n-m}, S^{n-m-1}\right)_{\mathbb{Q}}$. Set $u_{\mathrm{IP}}=e_{\mathrm{IP}}^{n-m} \times 1 \in\left(\Omega_{\mathrm{IP}}\right)^{n-m}(D \nu, S \nu)_{\mathbb{Q}}$, where $1 \in\left(\Omega_{\mathrm{IP}}\right)^{0}(Y)_{\mathbb{Q}}$ is the unit. The cap-product of $\mu:=$ $\left[\operatorname{id}_{\left(D^{n-m}, S^{n-m-1}\right)}\right] \in\left(\Omega_{\mathrm{IP}}\right)_{n-m}\left(D^{n-m}, S^{n-m}\right)_{\mathbb{Q}}$ with $e_{\mathrm{IP}}^{n-m}$ is given by

$$
e_{\mathrm{IP}}^{n-m} \cap \mu=(-1)^{s}\left[\mathrm{pt} \hookrightarrow D^{n-m}\right] \in\left(\Omega_{\mathrm{IP}}\right)_{0}\left(D^{n-m}\right)_{\mathbb{Q}},
$$


as we shall now verify. Set $\mu_{1}=\left[\operatorname{id}_{\left(D^{1}, S^{0}\right)}\right] \in\left(\Omega_{\mathrm{IP}}\right)_{1}\left(D^{1}, S^{0}\right)_{\mathbb{Q}}$. Then $\mu$ is the $(n-m)$-fold cross product

$$
\mu=\mu_{1} \times \cdots \times \mu_{1}
$$

and thus

$$
\begin{aligned}
e_{\mathrm{IP}}^{n-m} \cap \mu & =\left(e_{\mathrm{IP}} \times \cdots \times e_{\mathrm{IP}}\right) \cap\left(\mu_{1} \times \cdots \times \mu_{1}\right) \\
& =(-1)^{s_{1}}\left(e_{\mathrm{IP}} \cap \mu_{1}\right) \times \cdots \times\left(e_{\mathrm{IP}} \cap \mu_{1}\right),
\end{aligned}
$$

where $s_{1}=\frac{1}{2}(n-m)(n-m-1)$. Let $i:\left(S^{0}, \varnothing\right) \hookrightarrow\left(S^{0}, y_{-}\right)$be the canonical inclusion. To compute $e_{\mathrm{IP}} \cap \mu_{1}$, we consider the diagram

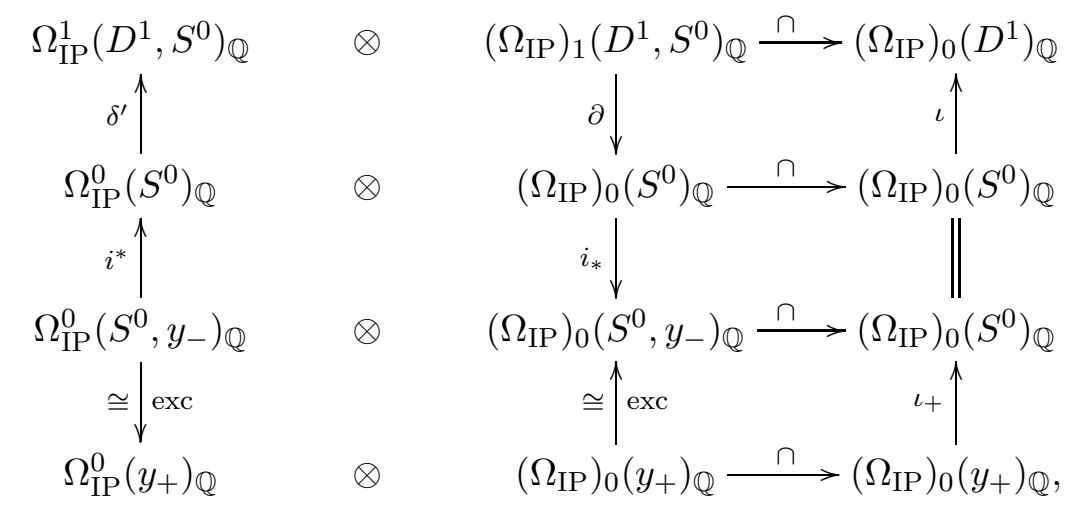

whose middle and bottom portion commute, while the top portion anticommutes, since

$$
\left(\delta^{\prime} a\right) \cap \alpha=(-1)^{\operatorname{deg}\left(\delta^{\prime} a\right)} a \cap \partial \alpha=-a \cap \partial \alpha
$$

for an IP-cobordism class $a$ of degree 0 . The image of $\mu_{1}$ under $i_{*} \partial$ is $\left[i:\left(S^{0}, \varnothing\right) \hookrightarrow\left(S^{0}, y_{-}\right)\right]$, while the image of $\left[\operatorname{id}_{y_{+}}\right] \in\left(\Omega_{\mathrm{IP}}\right)_{0}\left(y_{+}\right)$under the excision isomorphism is $\left[\left(y_{+}, \varnothing\right) \hookrightarrow\left(S^{0}, y_{-}\right)\right]$. Now

$$
\left[\left(y_{+}, \varnothing\right) \hookrightarrow\left(S^{0}, y_{-}\right)\right]=[i] \in\left(\Omega_{\mathrm{IP}}\right)_{0}\left(S^{0}, y_{-}\right)_{\mathbb{Q}}
$$

via the bordism $W=I \sqcup I$ (disjoint union of two intervals) and $F: W \rightarrow S^{0}$ defined by mapping the first copy of $I$ by the constant map to $y_{+}$and mapping the second copy of $I$ to $y_{-}$. Then the disjoint union $\left\{y_{+}\right\} \sqcup S^{0}$ is contained in $\partial W, F$ restricted to $\left\{y_{+}\right\} \sqcup S^{0}$ agrees with the disjoint union of the inclusion $y_{+} \hookrightarrow S^{0}$ and the identity map $S^{0} \rightarrow S^{0}$, while $F$ maps $\partial W-\left(\left\{y_{+}\right\} \sqcup S^{0}\right)$ to $y_{-}$. Hence $(W, F)$ is a valid bordism. Consequently,

$$
\begin{aligned}
e_{\mathrm{IP}} \cap \mu_{1} & =\delta^{\prime} i^{*} \operatorname{exc}^{-1}(1) \cap \mu_{1} \\
& =-\iota \iota_{+}\left(1 \cap \operatorname{exc}^{-1} i_{*} \partial\left(\mu_{1}\right)\right) \\
& =-\iota \iota_{+}\left(\operatorname{exc}^{-1} i_{*} \partial\left(\mu_{1}\right)\right) \\
& =-\iota \iota_{+}\left[\operatorname{id}_{y_{+}}\right] \\
& =-\left[\left\{y_{+}\right\} \hookrightarrow D^{1}\right]
\end{aligned}
$$


and so, with $s_{2}=n-m$,

$$
\begin{aligned}
e_{\mathrm{IP}}^{n-m} \cap \mu & =(-1)^{s_{1}}(-1)^{s_{2}}\left[\left\{y_{+}\right\} \hookrightarrow D^{1}\right] \times \cdots \times\left[\left\{y_{+}\right\} \hookrightarrow D^{1}\right] \\
& =(-1)^{s}\left[\mathrm{pt} \hookrightarrow D^{n-m}\right] .
\end{aligned}
$$

For any pair $(W, V)$ of IP-spaces, $n=\operatorname{dim} W$, let $\mathrm{Si}:\left(\Omega_{\mathrm{IP}}\right)_{n}(W, V)_{\mathbb{Q}} \rightarrow$ $\mathrm{L}_{n}^{\bullet}(W, V)_{\mathbb{Q}}$ be the composition

$$
\left(\Omega_{\mathrm{IP}}\right)_{n}(W, V) \stackrel{A}{\cong}\left(\mathbf{Q}_{\mathrm{IP}}\right)_{n}(W, V) \stackrel{\mathrm{Sig}}{\longrightarrow}\left(\mathbf{Q}_{\mathrm{rel}, \geq 0}^{\mathbb{Z}}\right)_{n}(W, V) \stackrel{\cong}{\longleftarrow} \mathrm{L}_{n}^{\bullet}(W, V)
$$

(which is just (9.3) in the absolute case), tensored with id $\mathbb{Q}$. By Section 13, we also have a corresponding multiplicative transformation of cohomology theories $\mathrm{Si}: \Omega_{\mathrm{IP}}^{*}(W, V) \rightarrow\left(\mathrm{L}^{\bullet}\right)^{*}(W, V)$. Thus the arguments used above for the transformation $S$ can be applied to Si to show that

$$
\operatorname{Si}\left(u_{\mathrm{IP}}\right)=u_{\mathbb{L}} \text {. }
$$

If $\phi: E \rightarrow F$ is any morphism of ring spectra, then the diagram

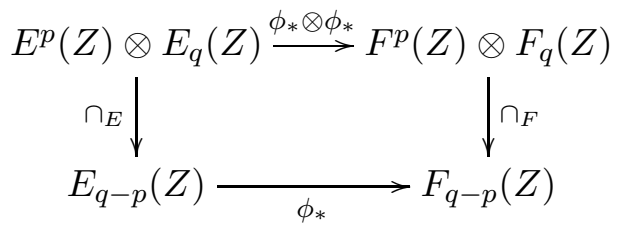

commutes (see e.g. Ada74, Prop. III.9.1 (v)].) Therefore, in view of (11.3) and (11.5), the following diagram commutes:

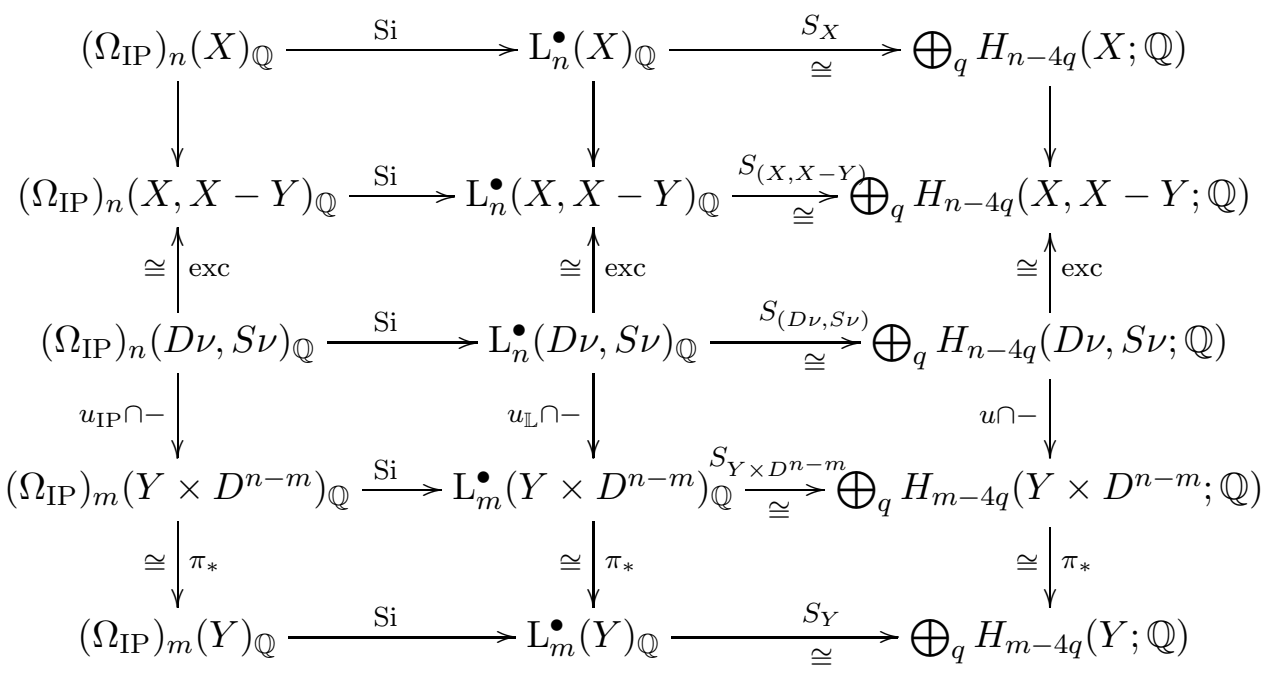

The left column, multiplied by $(-1)^{s}$, defines a map $j_{\mathrm{IP}}^{!}:\left(\Omega_{\mathrm{IP}}\right)_{n}(X)_{\mathbb{Q}} \rightarrow$ $\left(\Omega_{\mathrm{IP}}\right)_{m}(Y)_{\mathbb{Q}}$. The image of $[X]_{\mathrm{IP}}$ in $\left(\Omega_{\mathrm{IP}}\right)_{n}(X, X-Y)_{\mathbb{Q}}$ equals the image of $\left[\operatorname{id}_{(D \nu, S \nu)}\right] \in\left(\Omega_{\mathrm{IP}}\right)_{n}(D \nu, S \nu)_{\mathbb{Q}}$ under the excision isomorphism; the required bordism is given by the $\partial$-IP-space $W$ obtained from gluing the cylinder $X \times I$ to the cylinder $D \nu \times I$ along the canonical inclusion $D \nu \times\{1\} \hookrightarrow X \times\{0\}$. The map $F: W \rightarrow X$ is defined by $F(x, t)=x$ for $(x, t) \in X \times I$ and 
$(x, t) \in D \nu \times I$. Note that $F$ maps $\partial W-(X \times\{1\} \sqcup D \nu \times\{0\})$ to $X-Y$, whence $(W, F)$ is indeed a viable bordism. Using the cross product on IPbordism

$$
\left(\Omega_{\mathrm{IP}}\right)_{n-m}\left(D^{n-m}, S^{n-m-1}\right)_{\mathbb{Q}} \otimes\left(\Omega_{\mathrm{IP}}\right)_{m}(Y)_{\mathbb{Q}} \stackrel{\times}{\longrightarrow}\left(\Omega_{\mathrm{IP}}\right)_{n}(D \nu, S \nu)_{\mathbb{Q}},
$$

we may express the element $\left[\operatorname{id}_{(D \nu, S \nu)}\right]$ as

$$
\left[\operatorname{id}_{(D \nu, S \nu)}\right]=\mu \times[Y]_{\mathrm{IP}} .
$$

Thus, using (11.4), we find that

$$
\begin{aligned}
\pi_{*}\left(u_{\mathrm{IP}} \cap\left[\operatorname{id}_{(D \nu, S \nu)}\right]\right) & =\pi_{*}\left(\left(e_{\mathrm{IP}}^{n-m} \times 1\right) \cap\left(\mu \times[Y]_{\mathrm{IP}}\right)\right) \\
& =\pi_{*}\left((-1)^{\operatorname{deg}(1) \operatorname{deg}(\mu)}\left(e_{\mathrm{IP}}^{n-m} \cap \mu\right) \times\left(1 \cap[Y]_{\mathrm{IP}}\right)\right) \\
& =\pi_{*}\left(\left(e_{\mathrm{IP}}^{n-m} \cap \mu\right) \times\left(1 \cap[Y]_{\mathrm{IP}}\right)\right) \\
& =(-1)^{s} \pi_{*}\left(\left[\mathrm{pt} \hookrightarrow D^{n-m}\right] \times[Y]_{\mathrm{IP}}\right) \\
& =(-1)^{s}[Y]_{\mathrm{IP}} .
\end{aligned}
$$

This proves that

$$
j_{\mathrm{IP}}^{!}[X]_{\mathrm{IP}}=[Y]_{\mathrm{IP}}
$$

By the commutativity of the above diagram,

$$
j ! S_{X}[X]_{\mathbb{L}}=j ! S_{X} \operatorname{Si}[X]_{\mathrm{IP}}=S_{Y} \operatorname{Si} j_{\mathrm{IP}}^{!}[X]_{\mathrm{IP}}=S_{Y} \operatorname{Si}[Y]_{\mathrm{IP}}=S_{Y}[Y]_{\mathbb{L}},
$$

which proves property (1).

It remains to establish property (2). The map $f: X \rightarrow$ pt from $X$ to a point induces a homomorphism

$$
f_{*}: \bigoplus_{q} H_{n-4 q}(X ; \mathbb{Q}) \longrightarrow \bigoplus_{q} H_{n-4 q}(\mathrm{pt} ; \mathbb{Q})
$$

such that $\left(S_{X}[X]_{\mathbb{L}}\right)_{0}=f_{*} S_{X}[X]_{\mathbb{L}}$. If the dimension $n$ is not divisible by 4 , then $\bigoplus_{q} H_{n-4 q}(\mathrm{pt} ; \mathbb{Q})=0$ and thus $\epsilon_{*} f_{*} S_{X}[X]_{\mathbb{L}}=0=\sigma(X)$, that is, (2) holds. Assume that $n$ is divisible by 4 , so that $\bigoplus_{q} H_{n-4 q}(\mathrm{pt} ; \mathbb{Q})=H_{0}(\mathrm{pt} ; \mathbb{Q})$. Using the commutative diagram

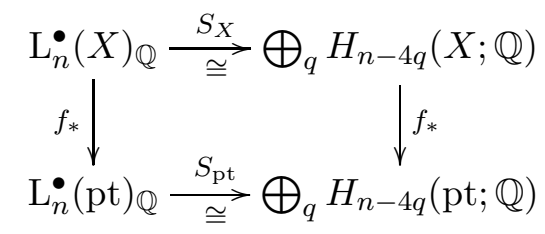

we can write

$$
f_{*} S_{X}[X]_{\mathbb{L}}=S_{\mathrm{pt}} f_{*}[X]_{\mathbb{L}} .
$$

Let $\{1\}=\pi_{1}(\mathrm{pt})$ denote the trivial fundamental group of the point. The associated assembly map

$$
\mathrm{L}_{n}^{\bullet}(\mathrm{pt})=\mathrm{L}_{n}^{\bullet}(B\{1\}) \stackrel{\alpha_{\{1\}}}{\longrightarrow} L^{n}(\mathbb{Z}[\{1\}])
$$


is an isomorphism. Recall ([Ran80, Prop. 7.2]) that when $n$ is divisible by 4 , there is an isomorphism $\sigma: L^{n}(\mathbb{Z}[\{1\}]) \cong \mathbb{Z}$ given by the signature $\sigma$ of a symmetric algebraic Poincaré complex. The diagram

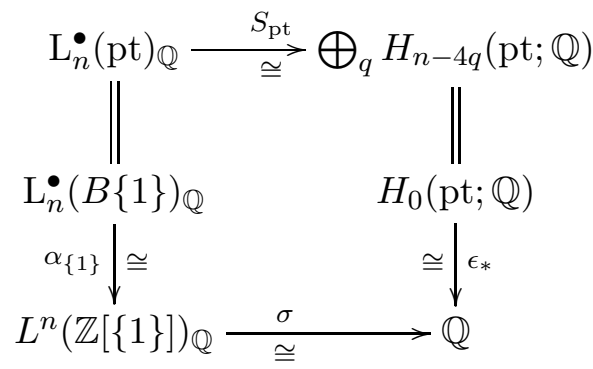

commutes, as the calculation

$$
\begin{aligned}
\epsilon_{*} S_{\mathrm{pt}}[\mathrm{pt}]_{\mathbb{L}} & =\epsilon_{*}\left(L^{*}(\mathrm{pt}) \cap[\mathrm{pt}]_{\mathbb{Q}}\right)=\epsilon_{*}\left(1 \cap[\mathrm{pt}]_{\mathbb{Q}}\right)=\epsilon_{*}[\mathrm{pt}]_{\mathbb{Q}}=1 \\
& =\sigma\left(\sigma^{*}(\mathrm{pt})\right)=\sigma\left(\alpha_{\{1\}}[\mathrm{pt}]_{\mathbb{L}}\right),
\end{aligned}
$$

using e.g. $\quad$ Ran92, Remark 16.17(i)], shows. (The formula $S_{M}[M]_{\mathbb{L}}=$ $L^{*}(M) \cap[M]_{\mathbb{Q}}$ holds for any closed smooth oriented $n$-manifold, where $L^{*}(M) \in H^{*}(M ; \mathbb{Q})$ is the Hirzebruch $L$-class and $[M]_{\mathbb{Q}} \in H_{n}(M ; \mathbb{Q})$ the rational fundamental class. Note that $\sigma\left(\sigma^{*}(\mathrm{pt})\right)=1$, since the composition

$$
\Omega_{0}^{\mathrm{SO}}(\mathrm{pt}) \stackrel{\sigma^{*}}{\longrightarrow} \mathrm{L}_{0}^{\bullet}(\mathrm{pt})=L^{0}(\mathbb{Z}) \stackrel{\sigma}{\longrightarrow} \mathbb{Z}
$$

is the signature homomorphism $\sigma: \Omega_{0}^{\mathrm{SO}}(\mathrm{pt}) \rightarrow \mathbb{Z}$, which sends the generator [id : pt $\rightarrow$ pt] to the signature of a point, which equals 1.) Using this diagram, we obtain

$$
\epsilon_{*} S_{\mathrm{pt}} f_{*}[X]_{\mathbb{L}}=\sigma \alpha_{\{1\}} f_{*}[X]_{\mathbb{L}} .
$$

Let $G=\pi_{1}(X)$ be the fundamental group of $X$ and $r: X \rightarrow B G$ a classifying map for the universal cover of $X$. The commutative diagram of spaces

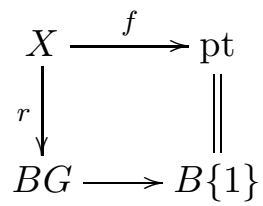

induces a commutative diagram

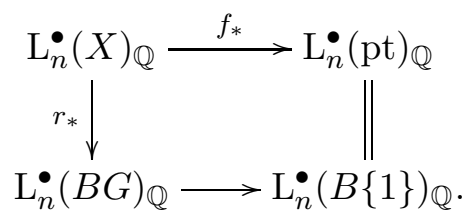

Since the assembly map, obtained e.g. by applying [WW95, Thm. 1.1] to the homotopy functor $Z \mapsto \mathbb{L}^{\bullet}\left(\mathbb{Z} \pi_{1}(Z)\right)$, is a natural transformation, the 
diagram

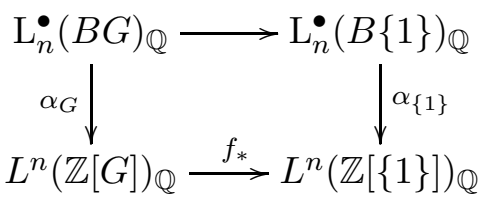

commutes. Using these two squares, we get

$$
f_{*} \alpha_{G} r_{*}[X]_{\mathbb{L}}=\alpha_{\{1\}} f_{*}[X]_{\mathbb{L}} .
$$

The ordinary signature information is contained in the symmetric signature by

$$
\sigma f_{*} \sigma_{\mathrm{IP}}^{*}(X)=\sigma(X)
$$

as follows from Remark 8.5. Putting equations (11.6), (11.7), (11.8) and (11.9) together, we compute

$$
\begin{aligned}
\epsilon_{*}\left(S_{X}[X]_{\mathbb{L}}\right)_{0} & =\epsilon_{*} f_{*} S_{X}[X]_{\mathbb{L}}=\epsilon_{*} S_{\mathrm{pt}} f_{*}[X]_{\mathbb{L}}=\sigma \alpha_{\{1\}} f_{*}[X]_{\mathbb{L}} \\
& =\sigma f_{*} \alpha_{G} r_{*}[X]_{\mathbb{L}}=\sigma f_{*} \sigma_{\mathrm{IP}}^{*}(X)=\sigma(X),
\end{aligned}
$$

as was to be shown.

Let $G=\pi_{1}(X)$ be the fundamental group and $r: X \rightarrow B G$ a classifying map for the universal cover of $X$. The map $r$ induces a homomorphism

$$
H_{*}(X ; \mathbb{Q}) \longrightarrow H_{*}(B G ; \mathbb{Q})
$$

on homology. The higher signatures of $X$ are the rational numbers

$$
\left\langle a, r_{*} L(X)\right\rangle, a \in H^{*}(B G ; \mathbb{Q}) .
$$

Theorem 11.2. Let $X$ be an $n$-dimensional compact IP-space whose fundamental group $G=\pi_{1}(X)$ satisfies the strong Novikov conjecture. Then the higher signatures of $X$ are stratified homotopy invariants.

Proof. Let $X$ and $X^{\prime}$ be $n$-dimensional compact IP-spaces with fundamental group $G$ and $f: X^{\prime} \rightarrow X$ an orientation preserving stratified homotopy equivalence. If $r: X \rightarrow B G$ is a classifying map for the universal cover of $X$, then $r^{\prime}=r \circ f: X^{\prime} \rightarrow B G$ is a classifying map for the universal cover of $X^{\prime}$. We must prove that

$$
r_{*}^{\prime} L\left(X^{\prime}\right)=r_{*} L(X) \in H_{*}(B G ; \mathbb{Q}) .
$$

By Theorem 9.3(ii), the assembly map

$$
\mathrm{L}_{n}^{\bullet}(X) \longrightarrow L^{n}(\mathbb{Z}[G])
$$

maps $[X]_{\mathbb{L}}$ to $\sigma_{\mathrm{IP}}^{*}(X)$. By naturality, the assembly map for the space $X$ factors through $B G$ (see also [Dav00, p. 216]):

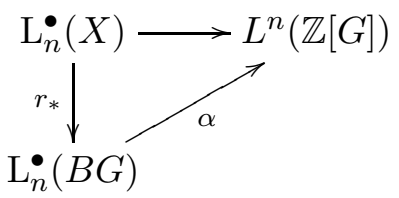


(similarly for $X^{\prime}$ ). Using this factorization, we may write

$$
\alpha r_{*}[X]_{\mathbb{L}}=\sigma_{\mathrm{IP}}^{*}(X), \alpha r_{*}^{\prime}\left[X^{\prime}\right]_{\mathbb{L}}=\sigma_{\mathrm{IP}}^{*}\left(X^{\prime}\right) .
$$

By the stratified homotopy invariance (8.2) of the IP symmetric signature,

$$
\sigma_{\mathrm{IP}}^{*}(X)=\sigma_{\mathrm{IP}}^{*}(r)=\sigma_{\mathrm{IP}}^{*}(r f)=\sigma_{\mathrm{IP}}^{*}\left(r^{\prime}\right)=\sigma_{\mathrm{IP}}^{*}\left(X^{\prime}\right) .
$$

As $\alpha$ is by assumption rationally injective, it follows that

$$
r_{*}[X]_{\mathbb{L}}=r_{*}^{\prime}\left[X^{\prime}\right]_{\mathbb{L}} \in \mathrm{L}_{n}^{\bullet}(B G) \otimes \mathbb{Q} \text {. }
$$

Using the commutative diagram

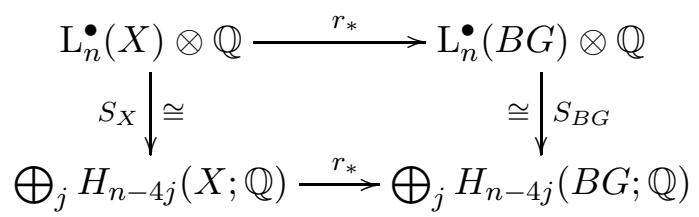

(and the analogous diagram for $X^{\prime}$ ), together with Lemma 11.1, we deduce

$$
\begin{aligned}
r_{*} L(X) & =r_{*} S_{X}[X]_{\mathbb{L}}=S_{B G} r_{*}[X]_{\mathbb{L}} \\
& =S_{B G} r_{*}^{\prime}\left[X^{\prime}\right]_{\mathbb{L}}=r_{*}^{\prime} S_{X^{\prime}}\left[X^{\prime}\right]_{\mathbb{L}}=r_{*}^{\prime} L\left(X^{\prime}\right) .
\end{aligned}
$$

An analytic version of Theorem 11.2 has been proven by Albin-LeichtnamMazzeo-Piazza in [ALMP13]. The scope of their theorem is in fact larger, as it applies even to those non-Witt spaces, for which a so-called analytic self-dual mezzoperversity exists. It was shown in [ABLMP13] that such perversity data corresponds topologically to the Lagrangian structures of Banagl as introduced in Ban02]. A comparison of the analytic argument to our argument shows that the role of our $\mathrm{L}_{n}^{\bullet}(X)$ is played in the analytic context by $K_{*}(X)$. The role of the isomorphisms $S_{X}$ is played by the Chern character. The group $L^{n}(\mathbb{Z}[G])$ corresponds to $K_{*}\left(C_{r}^{*} G\right)$, while our assembly map $\alpha$ corresponds to the assembly map $K_{*}(B G) \rightarrow K_{*}\left(C_{r}^{*} G\right)$ used in the analytic argument.

\section{Multiplicativity And COMmutativity}

Recall from Definition 6.1 that the symmetric signature map

$$
\text { Sig : } \mathbf{M}_{\mathrm{IP}} \rightarrow \mathbf{M}_{\mathrm{rel}, \geq 0}^{\mathbb{Z}}
$$

is the following composite in the homotopy category of spectra:

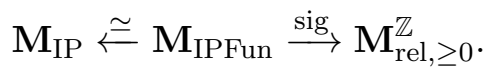

In this section we show that this composite is weakly equivalent to a composite of ring maps between commutative ring spectra. Specifically, we show the following. Recall Remarks 4.5 and 6.4 . 
Theorem 12.1. There are symmetric ring spectra $\mathbf{A}, \mathbf{B}$ and $\mathbf{C}$, a commutative symmetric ring spectrum $\mathbf{D}$, and a strictly commutative diagram

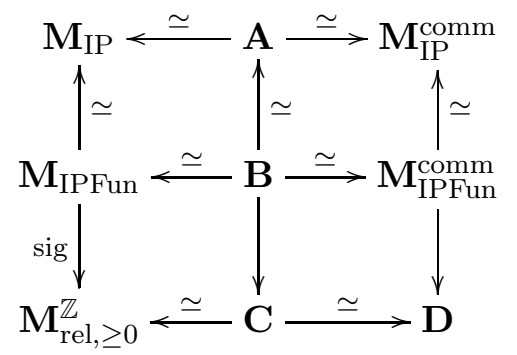

in which the horizontal arrows, the upper vertical arrows and the lower right vertical arrow are ring maps.

Remark 12.2. $\mathbf{D}$ is weakly equivalent to $\left(\mathbf{M}_{\mathrm{rel}, \geq 0}^{\mathbb{Z}}\right)^{\text {comm }}$ by $[\mathrm{LM}$, Remark 18.3].

The rest of this section is devoted to the proof of Theorem 12.1. The top half of the diagram has already been constructed in Remark 6.4. For the lower half we will use the method of the proof of [LM, Theorem 1.3] (it will be straightforward to check that the maps $\mathbf{M}_{\text {IPFun }} \leftarrow \mathbf{B} \rightarrow \mathbf{M}_{\text {IPFun }}^{\text {comm }}$ given by the proof in this section are the same as those given by Remark 6.4).

Remark 12.3. In order to apply the proof of [LM, Theorem 1.3] without change we would need to know (by analogy with the paragraph before [LM, Definition 15.5]) that the cross product gave a natural quasi-isomorphism from the functor

to the functor

$$
\left(\mathcal{A}_{\mathrm{IPFun}}\right)^{\times l} \stackrel{\text { sig } \times l}{\longrightarrow}\left(\mathcal{A}_{\mathrm{rel}}^{\mathbb{Z}}\right)^{\times l} \stackrel{\otimes}{\rightarrow} \mathcal{A}_{\mathrm{rel}}^{\mathbb{Z}}
$$

$$
\left(\mathcal{A}_{\text {IPFun }}\right)^{\times l} \rightarrow \mathcal{A}_{\text {IPFun }} \stackrel{\text { sig }}{\rightarrow} \mathcal{A}_{\text {rel }}^{\mathbb{Z}}
$$

(where the unmarked arrow is the product in $\mathcal{A}_{\text {IPFun }}$ ). But this is not the case, for the simple reason that the cross product does not give a map

$$
I S_{*}^{Q_{\bar{n}}, \bar{n}}(X \times X ; \mathbb{Z})^{\otimes l} \rightarrow I S_{*}^{Q_{\bar{n}, \bar{n}}}\left(X^{\times l} \times X^{\times l} ; \mathbb{Z}\right)
$$

(cf. [F, Lemma 6.4.1]). Our first task is to provide a suitable substitute, which will be given in Proposition 12.11.

Definition 12.4. Let $Y_{1}, \ldots, Y_{k}$ be stratified PL $\partial$-pseudomanifolds and give $Y_{1} \times \cdots \times Y_{k}$ the product stratification. Define a perversity $Q_{k}$ on $Y_{1} \times \cdots \times Y_{k}$ by

$$
Q_{k}\left(S_{1} \times \cdots \times S_{k}\right)=\left\{\begin{array}{l}
0 \text { if all } S_{i} \text { are regular, } \\
2 s-2+\sum \bar{n}\left(S_{i}\right) \text { otherwise }
\end{array}\right.
$$

where the $S_{i}$ are strata and $s$ is the number of $S_{i}$ that are singular.

In particular, $Q_{1}=\bar{n}$ and $Q_{2}=Q_{\bar{n}, \bar{n}}$. 
Lemma 12.5. The cross product induces a quasi-isomorphism $I S_{*}^{Q_{j}}\left(Y_{1} \times \cdots \times Y_{j} ; \mathbb{Z}\right) \otimes I S_{*}^{Q_{k}}\left(Y_{j+1} \times \cdots \times Y_{j+k} ; \mathbb{Z}\right) \rightarrow I S_{*}^{Q_{j+k}}\left(Y_{1} \times \cdots \times Y_{j+k} ; \mathbb{Z}\right)$.

This is immediate from [F, Theorem 6.4.6 and Remark 6.4.7]. We need a more general version of this.

Definition 12.6. (i) Let $A$ be a finite totally ordered set. A partition $\rho$ of $A$ is a collection $B_{1}, \ldots, B_{k}$ of disjoint subsets of $A$ such that $\cup B_{i}=A$ and $a<a^{\prime}$ whenever $a \in B_{i}, a^{\prime} \in B_{i^{\prime}}$ with $i<i^{\prime}$.

(ii) Let $X_{1}, \ldots, X_{l}$ be stratified PL $\partial$-pseudomanifolds and let

$$
\rho=\left\{B_{1}, \ldots, B_{k}\right\}
$$

be a partition of $\{1, \ldots, l\}$. Let $Y_{i}=\prod_{j \in B_{i}} X_{j}$ and give $Y_{i}$ the product stratification. Define

$$
I S_{*}^{\rho}\left(X_{1} \times \ldots \times X_{l} ; \mathbb{Z}\right)
$$

to be

$$
I S_{*}^{Q_{k}}\left(Y_{1} \times \ldots \times Y_{k} ; \mathbb{Z}\right) .
$$

Lemma 12.7. The cross product induces a quasi-isomorphism $I S_{*}^{\rho}\left(X_{1} \times \cdots \times X_{l} ; \mathbb{Z}\right) \otimes I S_{*}^{\rho^{\prime}}\left(X_{l+1} \times \cdots \times X_{l+m} ; \mathbb{Z}\right) \rightarrow I S_{*}^{\rho \cup \rho^{\prime}}\left(X_{1} \times \cdots \times X_{l+m} ; \mathbb{Z}\right)$.

This is immediate from Lemma 12.5

Definition 12.8. Let $\rho=\left\{B_{1}, \ldots, B_{j}\right\}$ and $\rho^{\prime}=\left\{C_{1}, \ldots, C_{k}\right\}$ be two partitions of a set $A$. Then $\rho^{\prime}$ is a refinement of $\rho$ if each $C_{i}$ is contained in some $B_{i}$.

Lemma 12.9. Let $\rho$ and $\rho^{\prime}$ be partitions of $\{1, \ldots, l\}$. If $\rho^{\prime}$ is a refinement of $\rho$ then

$$
I S_{*}^{\rho}\left(X_{1} \times \cdots \times X_{l} ; \mathbb{Z}\right) \subset I S_{*}^{\rho^{\prime}}\left(X_{1} \times \cdots \times X_{l} ; \mathbb{Z}\right)
$$

and the inclusion is a quasi-isomorphism.

Proof. First we claim that the perversity that gives $I S_{*}^{\rho}$ is $\leq$ the perversity that gives $I S_{*}^{\rho^{\prime}}$; the inclusion follows from this. It suffices to check the case where one piece in $\rho$ is divided into two pieces in $\rho^{\prime}$; this means that one of the factors $Y_{i}$ is replaced by two factors $Y^{\prime}$ and $Y^{\prime \prime}$. If $Y^{\prime}$ and $Y^{\prime \prime}$ are not both singular it's easy to verify the claim. Otherwise we need to show

$$
\bar{n}\left(Y^{\prime} \times Y^{\prime \prime}\right) \leq \bar{n}\left(Y^{\prime}\right)+\bar{n}\left(Y^{\prime \prime}\right)+2,
$$

and this follows by checking the cases where $\operatorname{codim} Y^{\prime}$ and $\operatorname{codim} Y^{\prime \prime}$ are both even, or both odd, or one is even.

To show the quasi-isomorphism it suffices to show that

$$
I S_{*}^{\bar{n}}\left(X_{1} \times \cdots \times X_{l} ; \mathbb{Z}\right) \hookrightarrow I S_{*}^{\rho}\left(X_{1} \times \cdots \times X_{l} ; \mathbb{Z}\right)
$$


is a quasi-isomorphism for every $\rho$. This in turn follows by induction from the following commutative diagram, where we let $\rho=\left\{B_{1}, \ldots, B_{k}\right\}$ and $\rho_{1}=\left\{B_{2}, \ldots, B_{k}\right\}$.

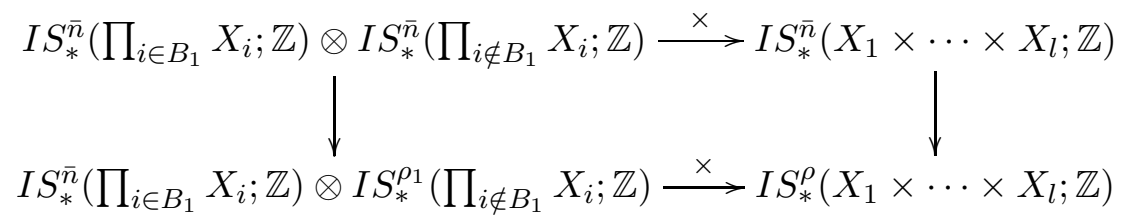

Here the horizontal arrows are quasi-isomorphisms by $[\mathrm{F}$, Theorem 6.4.6 and Remark 6.4.7] and the left vertical arrow is a quasi-isomorphism by the inductive hypothesis, so the right vertical arrow is a quasi-isomorphism as required.

Next is the analogue of Lemma 6.5 for this situation. Recall [LM, Definition 15.3(i)].

Lemma 12.10. (i) Let $l \geq 1$ and let $\rho=\left\{B_{1}, \ldots, B_{k}\right\}$ be a partition of $\{1, \ldots, l\}$. Let $\hat{\rho}$ be the partition $\left\{B_{1}, \ldots, B_{k}, B_{1}, \ldots, B_{k}\right\}$ of $\{1, \ldots, l\} \amalg\{1 \ldots, l\}$. Let $\left(X_{i}, \xi_{i}\right)$ for $1 \leq i \leq l$ be objects of $\mathcal{A}_{\mathrm{IPFun}}$. Give each $X_{i}$ the stratification of Proposition $A .2$ and give $X_{1} \times \cdots \times X_{l}$ and $\left(X_{1} \times \cdots \times X_{l}\right) \times\left(X_{1} \times \cdots \times X_{l}\right)$ the product stratifications. Let $\iota$ be the inclusion map

$I S_{*}^{Q \bar{n}, \bar{n}}\left(\left(X_{1} \times \cdots \times X_{l}\right) \times\left(X_{1} \times \cdots \times X_{l}\right) ; \mathbb{Z}\right) \subset I S_{*}^{\hat{\rho}}\left(\left(X_{1} \times \cdots \times X_{l}\right) \times\left(X_{1} \times \cdots \times X_{l}\right) ; \mathbb{Z}\right)$

given by Lemma 12.9. Then $(C, D, \beta, \varphi)$ is an object of $\mathcal{A}_{\text {rel }}^{\mathbb{Z}}$, where

$C=I S_{*}^{\rho}\left(X_{1} \times \cdots \times X_{l} ; \mathbb{Z}\right)$,

$D=I S_{*}^{\hat{\rho}}\left(\left(X_{1} \times \cdots \times X_{l}\right) \times\left(X_{1} \times \cdots \times X_{l}\right) ; \mathbb{Z}\right)$,

$\beta$ is the cross product followed by the inclusion $\iota$, and

$\varphi$ is the image of $\xi_{1} \times \cdots \times \xi_{l}$ under the map induced by the diagonal

$I S_{*}^{\overline{0}}\left(X_{1} \times \cdots \times X_{l} ; \mathbb{Z}\right) \rightarrow I S_{*}^{Q_{\bar{n}}, \bar{n}}\left(\left(X_{1} \times \cdots \times X_{l}\right) \times\left(X_{1} \times \cdots \times X_{l}\right) ; \mathbb{Z}\right)$

followed by $\iota$.

(ii) For $1 \leq i \leq l$, let $f_{i}:\left(X_{i}, \xi_{i}\right) \rightarrow\left(X_{i}^{\prime}, \xi_{i}^{\prime}\right)$ be a morphism in $\mathcal{A}_{\text {IPFun. }}$. Let $(C, D, \beta, \varphi)$ and $\left(C^{\prime}, D^{\prime}, \beta^{\prime}, \varphi^{\prime}\right)$ be the objects of $\mathcal{A}_{\mathrm{rel}}^{\mathbb{Z}}$ corresponding to the l-tuples $\left\{\left(X_{i}, \xi_{i}\right)\right\}$ and $\left\{\left(X_{i}^{\prime}, \xi_{i}^{\prime}\right)\right\}$. Then the $f_{i}$ induce a morphism $(C, D, \beta, \varphi) \rightarrow\left(C^{\prime}, D^{\prime}, \beta^{\prime}, \varphi^{\prime}\right)$.

Proof. Part (i) follows from Lemma 12.9 and the fact (shown in the proof of Lemma 6.5(i)) that $I S_{*}^{\bar{n}}\left(X_{1} \times \cdots \times X_{l} ; \mathbb{Z}\right)$ is homotopy finite. Part (ii) follows from the proof of Lemma 6.5)(ii).

Now we can give the statement promised in Remark 12.3. Let

$$
\operatorname{sig}_{\rho}:\left(\mathcal{A}_{\text {IPFun }}\right)^{\times l} \rightarrow \mathcal{A}_{\text {rel }}^{\mathbb{Z}}
$$

be the functor given by Lemma 12.10, and recall [LM, Definition 12.14]. 
Proposition 12.11. Let $\left\{B_{1}, \ldots, B_{k}\right\}$ be a partition of $\{1, \ldots, l\}$, let $\rho_{i}$ be a partition of $B_{i}$ for $1 \leq i \leq k$, let $\rho$ denote $\rho_{1} \cup \cdots \cup \rho_{k}$, and let $\rho^{\prime}$ be a refinement of $\rho$. There is a natural quasi-isomorphism from

$$
\left(\mathcal{A}_{\text {IPFun }}\right)^{\times l} \stackrel{\prod \operatorname{sig}_{\rho_{i}}}{\longrightarrow}\left(\mathcal{A}_{\text {rel }}^{\mathbb{Z}}\right)^{\times k} \stackrel{\otimes}{\rightarrow} \mathcal{A}_{\text {rel }}^{\mathbb{Z}}
$$

to

given by the maps

$$
\left(\mathcal{A}_{\text {IPFun }}\right)^{\times l} \stackrel{\operatorname{sig}_{\rho^{\prime}}}{\longrightarrow} \mathcal{A}_{\mathrm{rel}}^{\mathbb{Z}}
$$

$$
\begin{aligned}
I S_{*}^{\rho_{1}}\left(\prod_{i \in B_{1}} X_{i} ; \mathbb{Z}\right) \otimes & \cdots \otimes I S_{*}^{\rho_{k}}\left(\prod_{i \in B_{k}} X_{i} ; \mathbb{Z}\right) \\
& \stackrel{\times}{\rightarrow} I S_{*}^{\rho}\left(X_{1} \times \cdots \times X_{l} ; \mathbb{Z}\right) \rightarrow I S_{*}^{\rho^{\prime}}\left(X_{1} \times \cdots \times X_{l} ; \mathbb{Z}\right)
\end{aligned}
$$

and

$$
\begin{array}{r}
I S_{*}^{\hat{\rho_{1}}}\left(\left(\prod_{i \in B_{1}} X_{i}\right) \times\left(\prod_{i \in B_{1}} X_{i}\right) ; \mathbb{Z}\right) \otimes \cdots \otimes I S_{*}^{\hat{\rho_{k}}}\left(\left(\prod_{i \in B_{k}} X_{i}\right) \times\left(\prod_{i \in B_{k}} X_{i}\right) ; \mathbb{Z}\right) \\
\stackrel{\times}{\rightarrow} I S_{*}^{\widehat{\rho}}\left(\prod_{j=1}^{k}\left(\left(\prod_{i \in B_{f}} X_{i}\right) \times\left(\prod_{i \in B_{f}} X_{i}\right)\right) ; \mathbb{Z}\right) \cong I S_{*}^{\widehat{\rho}}\left(\left(\prod_{i=1}^{l} X_{i}\right) \times\left(\prod_{i=1}^{l} X_{i}\right) ; \mathbb{Z}\right) \\
\rightarrow I S_{*}^{\widehat{\rho^{\prime}}}\left(\left(\prod_{i=1}^{l} X_{i}\right) \times\left(\prod_{i=1}^{l} X_{i}\right) ; \mathbb{Z}\right) .
\end{array}
$$

This is immediate from Lemmas 12.7 and 12.9. Next we need the analogue of [LM, Definition 15.4]. Recall [LM, Definition 15.3(ii)].

Definition 12.12. Let $j \geq 0$ and let $r:\{1, \ldots, j\} \rightarrow\{u, v\}$. Let $\mathcal{A}_{i}$ denote $\mathcal{A}_{\text {IPFun }}$ if $r(i)=u$ and $\mathcal{A}_{\text {rel }}^{\mathbb{Z}}$ if $r(i)=v$.

(i) Let $1 \leq m \leq j$. A surjection

$$
h:\{1, \ldots, j\} \rightarrow\{1, \ldots, m\}
$$

is adapted to $r$ if $r$ is constant on each set $h^{-1}(i)$ and $h$ is injective on $r^{-1}(v)$.

(ii) Given a surjection

$$
h:\{1, \ldots, j\} \rightarrow\{1, \ldots, m\}
$$

which is adapted to $r$, and a partition $\rho_{i}$ of $h^{-1}(i)$ for $1 \leq i \leq m$, define

$$
\left(h, \rho_{1}, \ldots, \rho_{m}\right): \mathcal{A}_{1} \times \cdots \times \mathcal{A}_{j} \rightarrow\left(\mathcal{A}_{\mathrm{rel}}^{\mathbb{Z}}\right)^{\times m}
$$

by

$$
\left(h, \rho_{1}, \ldots, \rho_{m}\right) \bullet\left(x_{1}, \ldots, x_{j}\right)=\left(i^{\epsilon} y_{1}, \ldots, y_{m}\right),
$$

where $i^{\epsilon}$ is the sign that arises from putting the objects $x_{1}, \ldots, x_{j}$ into the order $x_{\theta(h)^{-1}(1)}, \ldots, x_{\theta(h)^{-1}(j)}$ and

$$
y_{i}= \begin{cases}\operatorname{sig}_{\rho_{i}}\left(\left\{x_{p}\right\}_{p \in h^{-1}(i)}\right) & \text { if } h^{-1}(i) \subset r^{-1}(u), \\ x_{h^{-1}(i)} & \text { if } h^{-1}(i) \in r^{-1}(v) .\end{cases}
$$


(iii) A datum of type $r$ is a tuple

$$
\left(h, \rho_{1}, \ldots, \rho_{m}, \eta\right)
$$

where $h$ is a surjection which is adapted to $r, \rho_{i}$ is a partition of $h^{-1}(i)$, and $\eta$ is an element of $\Sigma_{j}$ with the property that $h \circ \eta=h$.

(iv) Given a datum

$$
\mathbf{d}=\left(h, \rho_{1}, \ldots, \rho_{m}, \eta\right)
$$

of type $r$, define

$$
\mathrm{d} \boldsymbol{\Xi}: \mathcal{A}_{1} \times \cdots \times \mathcal{A}_{j} \rightarrow \mathcal{A}_{\mathrm{rel}}^{\mathbb{Z}}
$$

to be the composite

$$
\begin{aligned}
\mathcal{A}_{1} \times \cdots \times \mathcal{A}_{j} \stackrel{\eta}{\rightarrow} \mathcal{A}_{\eta^{-1}(1)} \times \cdots \times \mathcal{A}_{\eta^{-1}(j)}=\mathcal{A}_{1} \times \cdots \times \mathcal{A}_{j} \\
\stackrel{\left(h, \rho_{1}, \ldots, \rho_{m}\right)}{\longrightarrow}\left(\mathcal{A}_{\mathrm{rel}}^{\mathbb{Z}}\right)^{\times m} \stackrel{\otimes}{\rightarrow} \mathcal{A}_{\mathrm{rel}}^{\mathbb{Z}},
\end{aligned}
$$

where $\eta$ permutes the factors with the usual sign.

Finally, we have the analogue of [LM, Definition 15.5].

Definition 12.13. For data of type $r$, define

$$
\left(h, \rho_{1}, \ldots, \rho_{m}, \eta\right) \leq\left(h^{\prime}, \rho_{1}^{\prime}, \ldots, \rho_{m^{\prime}}^{\prime}, \eta^{\prime}\right)
$$

if for each $i \in\{1, \ldots, m\}$ there is a $p \in\left\{1, \ldots, m^{\prime}\right\}$ such that $\eta^{-1}\left(h^{-1}(i)\right)$ is contained in $\eta^{\prime-1}\left(h^{\prime-1}(p)\right)$ and $\eta^{\prime} \eta^{-1}$ takes each piece of the partition $\rho_{i}$ to a union of pieces of the partition $\rho_{p}^{\prime}$.

With these changes, the proof of [LM, Theorem 1.3] goes through to construct the lower half of Diagram (12.1). This completes the proof of Theorem 12.1.

\section{Multiplicativity of the $L$-TheORY Fundamental Class}

In this section we prove

Theorem 13.1. Let $X$ and $Y$ be compact oriented IP spaces. Then

$$
[X \times Y]_{\mathbb{L}}=[X]_{\mathbb{L}} \times[Y]_{\mathbb{L}} .
$$

Remark 13.2. We will use the results of Section 12 , but one could give a simpler proof of Theorem 13.1 using only part of the machinery of Section 12 .

The first step in the proof of Theorem 13.1 is to observe that we can replace the spectra $\mathbf{Q}$ in Definition 9.6 by the equivalent symmetric spectra M. Each of the symmetric spectra $\mathbf{M}_{\mathrm{IP}, Z}, Z_{+} \wedge \mathbf{M}_{\mathrm{IP}}, Z_{+} \wedge \mathbf{M}_{\mathrm{rel}, \geq 0}^{\mathbb{Z}}$ and $Z_{+} \wedge \mathbf{M}_{\geq 0}^{\mathbb{Z}}$ is semistable ([HSS00, Definition 5.6.1]) by [LM13, Corollary 17.9(i)] and [Sch08, Examples 4.2 and 4.7], and hence their "true" (i.e., derived) homotopy groups agree with their homotopy groups by Sch08, 
Example 5.5]. Thus for a compact oriented IP space $Z$ of dimension $l$ the class $[Z]_{\mathbb{L}}$ is the image of $[Z]_{\mathrm{IP}}$ under the composite

$\left(\Omega_{\mathrm{IP}}\right)_{l}(Z) \stackrel{\cong}{\rightrightarrows} \pi_{l} \mathbf{M}_{\mathrm{IP}, Z} \stackrel{\alpha}{\stackrel{\alpha}{\cong}} \pi_{l}\left(Z_{+} \wedge \mathbf{M}_{\mathrm{IP}}\right) \stackrel{\mathrm{Sig}}{\longrightarrow} \pi_{l}\left(Z_{+} \wedge \mathbf{M}_{\mathrm{rel}, \geq 0}^{\mathbb{Z}}\right) \stackrel{\cong}{\leftrightarrows} \pi_{l}\left(Z_{+} \wedge \mathbf{M}_{\geq 0}^{\mathbb{Z}}\right)$

(where the first map is given by Remark 3.1] and [LM13, Proposition 17.7]).

Next we observe that the functors in (13.1) have product operations. For the first functor (and for any spaces $X$ and $Y$ ), Cartesian product induces a map

$$
\left(\Omega_{\mathrm{IP}}\right)_{m}(X) \otimes\left(\Omega_{\mathrm{IP}}\right)_{n}(Y) \rightarrow\left(\Omega_{\mathrm{IP}}\right)_{m+n}(X \times Y)
$$

by [F, Lemma 2.11.7]. For the second functor, Cartesian product induces

$$
\mathcal{A}_{\mathrm{IP}, X} \times \mathcal{A}_{\mathrm{IP}, Y} \rightarrow \mathcal{A}_{\mathrm{IP}, X \times Y}
$$

and this induces a map

$$
\mathbf{M}_{\mathrm{IP}, X} \wedge \mathbf{M}_{\mathrm{IP}, Y} \rightarrow \mathbf{M}_{\mathrm{IP}, X \times Y}
$$

which gives the desired product. The third, fourth and fifth functors in (13.1) have products because $\mathbf{M}_{\mathrm{IP}}, \mathbf{M}_{\mathrm{rel}, \geq 0}^{\mathbb{Z}}$ and $\mathbf{M}_{\geq 0}^{\mathbb{Z}}$ are ring spectra.

It therefore suffices to show that the maps in the composite (13.1) preserve products. For the second map this follows from Proposition F.1, for the third map from Theorem 12.1, and for the fourth map from [LM, Remark 13.2]. We will denote the first map by $\chi$, so it remains to show

Lemma 13.3. The map

$$
\chi:\left(\Omega_{\mathrm{IP}}\right)_{*}(Z) \stackrel{\cong}{\rightrightarrows} \pi_{*} \mathbf{M}_{\mathrm{IP}, Z}
$$

preserves products.

The rest of this section gives the proof of this lemma.

Recall that for a spectrum $\mathbf{Q}$ or a symmetric spectrum $\mathbf{M}$ we write $Q_{k}$ and $M_{k}$ for the $k$-th space. The map $\chi$ can be written as the composite

$$
\left(\Omega_{\mathrm{IP}}\right)_{l}(Z) \stackrel{\cong}{\rightarrow} \pi_{l}\left(Q_{\mathrm{IP}, Z}\right)_{0} \rightarrow \pi_{l+k}\left(Q_{\mathrm{IP}, Z}\right)_{k} \rightarrow \pi_{l+k}\left(M_{\mathrm{IP}, Z}\right)_{k} \rightarrow \pi_{l} \mathbf{M}_{\mathrm{IP}, Z}
$$

for $k \geq 1$, where the first arrow is given by Remark [3.1, the second is the suspension map ([LM13, page 44] and Appendix G), the third is described in [LM13, top of page 53], and the fourth is given by the definition of the homotopy groups of a spectrum.

If $f: W \rightarrow Z$ is a map from an $l$-dimensional compact oriented IP space to $Z$, and if $p \leq l+2$, let us write $f^{[l+1]}$ (resp., $f^{[p, l+2-p]}$ ) for the $\Delta^{l+1}$-ad (resp., $\Delta^{p} \times \Delta^{l+2-p_{-a d}}$ ) which takes the top cell with its canonical orientation to $f$ and all other cells to $\emptyset \rightarrow Z$. Then $f^{[l+1]}$ is a simplex in $\left(M_{\mathrm{IP}, Z}\right)_{1}$ with all faces at the basepoint, so it determines an element of $\pi_{l+1}\left(M_{\mathrm{IP}, Z}\right)_{1}$ which we will denote by $\underline{f}^{[l+1]}$. Similarly, $f^{[p, l+2-p]}$ determines 
an element of $\pi_{l+2}\left(M_{\mathrm{IP}, Z}\right)_{2}$ which we will denote by $f^{[p, l+2-p]}$. From [LM13, Sections 15 and 17] (but using the signs in Appendix G) we see that

$$
\chi([f]) \text { is represented by } \underline{f}^{[l+1]} \in \pi_{l+1}\left(M_{\mathrm{IP}, Z}\right)_{1}
$$

and

$$
\chi([f]) \text { is represented by }-\underline{f}^{[0, l+2]} \in \pi_{l+2}\left(M_{\mathrm{IP}, Z}\right)_{2} .
$$

Now let $g: U \rightarrow X$ and $h: V \rightarrow Y$ be maps from compact oriented IP spaces of dimensions $m$ and $n$ respectively; we need to show that

$$
\chi([g]) \chi([h])=\chi([g \times h]) .
$$

By (13.3) and the proof of [Sch, Theorem I.4.54], $\chi([g]) \chi([h])$ is represented by the composite

$$
\begin{array}{r}
S^{1} \wedge S^{1} \wedge S^{m} \wedge S^{n} \rightarrow S^{1} \wedge S^{m} \wedge S^{1} \wedge S^{n} \stackrel{\underline{g}^{[m+1]} \wedge \underline{\underline{n}}^{[n+1]}}{\longrightarrow}\left(M_{\mathrm{IP}, X}\right)_{1} \wedge\left(M_{\mathrm{IP}, Y}\right)_{1} \\
\rightarrow\left(M_{\mathrm{IP}, X \times Y}\right)_{2}
\end{array}
$$

(cf. [Sch, I.4.55]). By [LM13, Section 18] this composite is equal to $-(g \times h)^{[m+1, n+1]}$ (for the sign, note that the first map has degree $(-1)^{m}$ and the last map includes a sign of $(-1)^{m+1}$ by [LM13, Remark 18.3]) so by (13.4) the proof of (13.5) reduces to showing that $(g \times h)^{[m+1, n+1]}=$

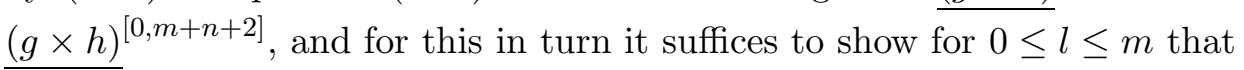

$$
\underline{(g \times h)}^{[m+1-l, n+1+l]}=\underline{(g \times h)}^{[m-l, n+2+l]} .
$$

To prove (13.6), let $F$ be the $\left(\Delta^{m+1-l} \times \Delta^{n+2+l}\right)$-ad which takes

- the top cell (with its canonical orientation) to the composite

$$
U \times V \times I \rightarrow U \times V \stackrel{g \times h}{\longrightarrow} X \times Y
$$

(where the first map is the projection),

- the cell $\partial_{0} \Delta^{m+1-l} \times \Delta^{n+2+l}$ (with its canonical orientation) to $(-1)^{m+n}$ times

$$
U \times V \times\{1\} \rightarrow U \times V \stackrel{g \times h}{\longrightarrow} X \times Y,
$$

- the cell $\Delta^{m+1-l} \times \partial_{0} \Delta^{n+2+l}$ (with its canonical orientation) to $(-1)^{n-l}$ times

$$
U \times V \times\{0\} \rightarrow U \times V \stackrel{g \times h}{\longrightarrow} X \times Y,
$$

- and all other cells to the map $\emptyset \rightarrow X \times Y$.

Then $F$ determines a map

$$
\Phi: \Delta^{m+1-l} \times \Delta^{n+1+l+1} \rightarrow\left(M_{\mathrm{IP}, X \times Y}\right)_{2},
$$

and the restriction of $\Phi$ to the boundary of $\Delta^{m+1-l} \times \Delta^{n+2+l}$ (which is nullhomotopic) is easily seen (using the signs in Appendix F below) to 
be $(-1)^{m+n}(g \times h)^{[m-l, n+2+l]}+(-1)^{m+1+n}(g \times h)^{[m+1-l, n+1+l]}$; this proves (13.6) and completes the proof of Lemma 13.3

\section{Proof of Theorem 9.5}

By [WW95, Observation 1.3] it suffices to show that $\Phi$ is strongly excisive. The fact that $\left(\Omega_{\mathrm{IP}}\right)_{*}$ is a strongly additive homology theory implies that, for any collection of spaces $\left\{X_{\alpha}\right\}$, the canonical map

$$
\begin{array}{r}
\pi_{*}\left(\bigvee \Phi\left(X_{\alpha}\right)\right) \cong \bigoplus \pi_{*}\left(\Phi\left(X_{\alpha}\right)\right) \cong \bigoplus \Omega_{\mathrm{IP} *}\left(X_{\alpha}\right) \longrightarrow \Omega_{\mathrm{IP} *}\left(\bigvee X_{\alpha}\right) \\
\cong \pi_{*}\left(\Phi\left(\bigvee X_{\alpha}\right)\right)
\end{array}
$$

is an equivalence. Hence, $\Phi$ preserves arbitrary coproducts and it suffices to show that $\Phi$ preserves homotopy cocartesian squares. First we observe that $\Phi$ takes monomorphisms to cofibrations in the level model structure given by [MMSS01, Theorem 6.5] (because a monomorphism $Z \rightarrow Z^{\prime}$ gives a map from the $k$-th space of $\mathbf{Q}_{\mathrm{IP}, Z}$ to the $k$-th space of $\mathbf{Q}_{\mathrm{IP}, Z^{\prime}}$ which is the inclusion of a sub-CW-complex). For a based space $W$, let

$$
\bar{\Phi}(W)=\Phi(W) / \Phi(*) \text {. }
$$

Then the natural map

$$
\Phi(W) \rightarrow \bar{\Phi}\left(W_{+}\right)
$$

(where + denotes a disjoint basepoint) is a weak equivalence because $\left(\Omega_{\mathrm{IP}}\right)_{*}$ is a homology theory and thus

$$
\pi_{*} \Phi(W) \cong \Omega_{\mathrm{IP} *}(W) \cong \Omega_{\mathrm{IP} *}\left(W_{+}\right) / \Omega_{\mathrm{IP} *}(*) \cong \pi_{*} \bar{\Phi}\left(W_{+}\right) .
$$

It therefore suffices to show that $\bar{\Phi}$ takes homotopy cocartesian squares of based spaces to homotopy cocartesian squares of spectra.

As a first step we give a relationship between $\Sigma \bar{\Phi}(W)$ and $\bar{\Phi}(\Sigma W)$. Let $C W$ be the cone $I \wedge W$, where 1 is the basepoint of $I$, and let $S(W)$ denote the pushout of the diagram

$$
\bar{\Phi}(C W) \leftarrow \bar{\Phi}(W) \rightarrow \bar{\Phi}(C W) .
$$

Since $\bar{\Phi}(C Z)$ is contractible, and since $\bar{\Phi}$ takes monomorphisms to cofibrations in the level model structure, $S(W)$ is weakly equivalent to $\Sigma \bar{\Phi}(W)$. Since $\Sigma W$ is the pushout of

$$
C W \leftarrow W \rightarrow C W
$$

there is an evident map

$$
\mathfrak{S}: S(W) \rightarrow \bar{\Phi}(\Sigma W) .
$$

Lemma 14.1. S is a weak equivalence for all $W$.

We defer the proof for a moment. Let

$$
B \stackrel{i}{\leftarrow} A \stackrel{j}{\rightarrow} C
$$


be a diagram of based spaces. The homotopy pushout of this diagram is the pushout of the diagram

$$
M i \hookleftarrow A \hookrightarrow M j
$$

where $M i$ and $M j$ are the mapping cylinders; we will denote this pushout by $D$. The homotopy pushout of

$$
\bar{\Phi}(B) \stackrel{\bar{\Phi}(i)}{\longleftarrow} \bar{\Phi}(A) \stackrel{\bar{\Phi}(j)}{\longrightarrow} \bar{\Phi}(C)
$$

is (up to weak equivalence) the pushout, which we will denote by $E$, of

$$
\bar{\Phi}(M i) \hookleftarrow \bar{\Phi}(A) \hookrightarrow \bar{\Phi}(M j) .
$$

It therefore suffices to show that the map $E \rightarrow \bar{\Phi}(D)$ is a weak equivalence. Consider the diagram

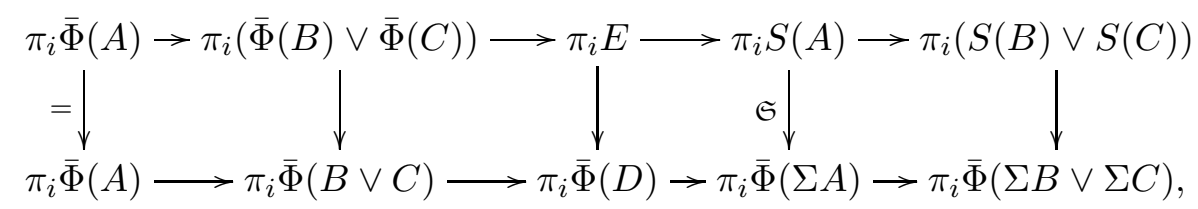

where the rightmost vertical arrow is induced by the maps

$$
S(B) \stackrel{\mathfrak{S}}{\rightarrow} \bar{\Phi}(\Sigma B) \rightarrow \bar{\Phi}(\Sigma B \vee \Sigma C)
$$

and

$$
S(C) \stackrel{\mathfrak{S}}{\rightarrow} \bar{\Phi}(\Sigma C) \rightarrow \bar{\Phi}(\Sigma B \vee \Sigma C) .
$$

The top row of the diagram is exact because it is $\pi_{i}$ of a cofiber sequence. The fact that $\left(\Omega_{\mathrm{IP}}\right)_{*}$ is a homology theory implies that the second row of the diagram is exact (because it is $\pi_{i} \bar{\Phi}$ of a cofiber sequence), and also that the second vertical arrow is an isomorphism. The fourth and fifth vertical arrows are isomorphisms by Lemma 14.1, and hence the middle vertical arrow is an isomorphism as required.

It remains to prove Lemma 14.1. We begin by describing a suspension map

$$
s: \pi_{i} \bar{\Phi}(W) \rightarrow \pi_{i+1} S(W) .
$$

Let $a \in \pi_{i} \bar{\Phi}(W)$. Let $\kappa_{i}(W)$ denote the kernel of the map $\pi_{i} \Phi(W) \rightarrow \pi_{i} \Phi(*)$; then $\kappa_{i}(W) \rightarrow \pi_{i} \bar{\Phi}(W)$ is an isomorphism (because the short exact sequence $\pi_{i} \Phi(*) \rightarrow \pi_{i} \Phi(W) \rightarrow \pi_{i} \bar{\Phi}(W)$ is split by the map $\left.W \rightarrow *\right)$, so $a$ comes from an element $\tilde{a} \in \kappa_{i}(W)$. Let $\Phi_{0}(W)$ denote the 0 -th space of the spectrum $\Phi(W)$ ([LM13, Definitions 15.8 and 15.4]). Since $\Phi(W)$ is an $\Omega$ spectrum ([LM13, Proposition 15.9]) and $\Phi_{0}(W)$ is a Kan complex ([LM13, Lemma 15.12]), $\tilde{a}$ is represented by an $i$-simplex $\sigma$ of $\Phi_{0}(W)$ with all faces at the basepoint. Let $\sigma^{\prime}$ be the image of $\sigma$ in $\Phi_{0}(C W)$; then $\sigma^{\prime}$ represents an element of $\kappa_{i}(C W)$. Since $\kappa_{i}(C W)=0$, there is an $(i+1)$-simplex $\tau$ of $\Phi_{0}(C W)$ with $\partial_{0}(\tau)=\sigma$ and all other faces at the basepoint. Let $\tau_{1}$ and $\tau_{2}$ be the images of $\tau$ under the two inclusions of $\bar{\Phi}_{0}(C W)$ into the 0 -th space of $S(W)$ and let

$$
D=\Delta^{i} \cup_{d^{0} \Delta^{i}} \Delta^{i}
$$


then $\tau_{1}$ and $\tau_{2}$ give a map

$$
\mathbf{a}: D / \partial D \rightarrow S(W)
$$

which represents $s(a)$.

Combining the map $s$ with the weak equivalence between $S(W)$ and $\Sigma \bar{\Phi}(W)$ gives the usual suspension map $\pi_{i} \bar{\Phi}(W) \rightarrow \pi_{i+1} \Sigma \bar{\Phi}(W)$, as the reader can verify, so $s$ is an isomorphism.

It therefore suffices to show that the composite

$$
\begin{array}{r}
\left(\Omega_{\mathrm{IP}}\right)_{i}(W, *) \cong \operatorname{ker}\left(\left(\Omega_{\mathrm{IP}}\right)_{i}(W) \rightarrow\left(\Omega_{\mathrm{IP}}\right)_{i}(*)\right) \cong \kappa_{i}(W) \cong \pi_{i} \bar{\Phi}(W) \\
\stackrel{s}{\rightarrow} \pi_{i+1} S(W) \stackrel{\mathfrak{S}}{\rightarrow} \pi_{i+1} \bar{\Phi}(\Sigma W) \cong\left(\Omega_{\mathrm{IP}}\right)_{i+1}(\Sigma W, *)
\end{array}
$$

is an isomorphism. We will show that this composite is equal to the suspension isomorphism

$$
s^{\prime}:\left(\Omega_{\mathrm{IP}}\right)_{i}(W, *) \rightarrow\left(\Omega_{\mathrm{IP}}\right)_{i+1}(\Sigma W, *)
$$

of the homology theory $\left(\Omega_{\mathrm{IP}}\right)_{*}$. First we give an explicit description of the composite (14.2). Let $b \in\left(\Omega_{\mathrm{IP}}\right)_{i}(W, *)$ and let $\tilde{b}$ be the corresponding element of $\operatorname{ker}\left(\left(\Omega_{\mathrm{IP}}\right)_{i}(W) \rightarrow\left(\Omega_{\mathrm{IP}}\right)_{i}(*)\right)$. Then $\tilde{b}$ is represented by a map $f: M \rightarrow W$ where $M$ is an $i$-dimensional IP-space which is a boundary; say $M=\partial N$. Recall that $k$-simplices of $\Phi_{0}(W)$ are the same thing as elements of $\operatorname{ad}_{\mathrm{IP}, W}^{0}\left(\Delta^{k}\right)$. Let $\sigma$ be the $i$-simplex of $\Phi_{0}(W)$ which takes $\Delta^{i}$ to $f$ and all faces of $\Delta^{i}$ to $\emptyset \rightarrow W$, and let $\sigma^{\prime}$ be the image of $\sigma$ in $\Phi_{0}(C W)$. We can construct an $(i+1)$-simplex $\tau$ of $\Phi_{0}(C W)$ with $\partial_{0}(\tau)=\sigma^{\prime}$ and all other faces at the basepoint as follows. Let $P$ be

$$
(I \times M) \cup_{1 \times M} N
$$

let $g: P \rightarrow C W$ take $(t, x) \in I \times M$ to $[t, f(x)] \in C W$ and $N$ to $[1, *]$, and finally let $\tau$ take $\Delta^{i+1}$ to $g, d^{0} \Delta^{i+1}$ to $f$, and the remaining faces to $\emptyset \rightarrow W$. Let $\tau_{1}$ and $\tau_{2}$ be the images of $\tau$ under the two maps $\Phi_{0}(C W) \rightarrow \bar{\Phi}_{0}(\Sigma W)$; then (with the notation of Equation (14.1) $) \tau_{1}$ and $\tau_{2}$ give a map

$$
\mathbf{b}: D / \partial D \rightarrow \bar{\Phi}_{0} \Sigma W
$$

which represents an element of $\pi_{i+1} \bar{\Phi}(\Sigma W)$, and the image of this element in $\left(\Omega_{\mathrm{IP}}\right)_{i+1}(\Sigma W, *)$ is the image of $b$ under the composite (14.2).

Next we show that this description of the image of $b$ in $\pi_{i+1} \bar{\Phi}(\Sigma W)$ can be simplified. Let the two copies of $C W$ in $\Sigma W$ be $[-1,0] \wedge W$ and $[0,1] \wedge W$, where the basepoints of $[-1,0]$ and $[0,1]$ are -1 and 1 . Let

$$
Q=N \cup_{-1 \times M}([-1,1] \times M) \cup_{1 \times M} N,
$$

and let $h: Q \rightarrow \Sigma W$ take $(t, x)$ to $[t, f(x)]$ and both copies of $N$ to the basepoint. Let $\tau_{3}$ be the $(i+1)$-simplex of $\Phi_{0}(\Sigma W)$ which takes $\Delta^{i+1}$ to $h$ and all faces of $\Delta^{i+1}$ to $\emptyset \rightarrow \Sigma W$; then $\tau_{3}$ gives a map

$$
\mathbf{b}^{\prime}: \Delta^{i+1} / \partial \Delta^{i+1} \rightarrow \bar{\Phi}(\Sigma W),
$$

and we claim that $\mathbf{b}$ and $\mathbf{b}^{\prime}$ represent the same element of $\pi_{i+1} \bar{\Phi}(\Sigma W)$. To see this, let $v$ be the $(i+2)$-simplex of $\Phi_{0}(\Sigma W)$ such that 
- $v\left(\Delta^{i+2}\right)$ is the composite

$$
I \times Q \stackrel{p}{\rightarrow} Q \stackrel{h}{\rightarrow} \Sigma W
$$

(where $p$ is the projection),

- $v\left(d^{0} \Delta^{i+2}\right)=\left.h \circ p\right|_{0 \times Q}$,

- $v\left(d^{1} \Delta^{i+2}\right)=\left.h \circ p\right|_{1 \times\left(N \cup_{-1 \times M}([-1,0] \times M)\right)}$,

- $v\left(d^{2} \Delta^{i+2}\right)=\left.h \circ p\right|_{1 \times\left(([0,1] \times M) \cup_{1 \times M} N\right)}$,

- $v$ takes all other faces to $\emptyset \rightarrow \Sigma W$.

Then $v$ gives a homotopy between $\mathbf{b}$ and $\mathbf{b}^{\prime}$ which verifies the claim.

The element of $\left(\Omega_{\mathrm{IP}}\right)_{i+1}(\Sigma W, *)$ corresponding to $\mathbf{b}^{\prime}$ is represented by the map $h: Q \rightarrow \Sigma W$; this completes our calculation of the image of $b$ under the composite (14.2).

Next we claim that the image of $b$ under the map (14.3) is represented by $\left.h\right|_{Q^{\prime}}$, where

$$
Q^{\prime}=([-1,1] \times M) \cup_{1 \times M} N .
$$

To see this, recall that the suspension map $s^{\prime}$ is defined to be the inverse of the composite

$$
\left(\Omega_{\mathrm{IP}}\right)_{i+1}(\Sigma W, *) \underset{\cong}{\stackrel{q}{\cong}}\left(\Omega_{\mathrm{IP}}\right)_{i+1}\left(C^{\prime} W, W\right) \stackrel{\partial}{\rightarrow}\left(\Omega_{\mathrm{IP}}\right)_{i}(W, *),
$$

where $C^{\prime} W=[-1,1] \wedge W$ (with the basepoint of $[-1,1]$ at 1$), q$ is the quotient map, and $\partial$ is the boundary map of the homology theory $\left(\Omega_{\mathrm{IP}}\right)_{*}$. Now consider the map

$$
k: Q^{\prime} \rightarrow C^{\prime} W
$$

which takes $(t, x)$ to $[t, f(x)]$ and $N$ to the basepoint. Recall from Par90, Section 5] that the boundary map $\partial$ is defined as in [C79, Section 4]; thus $\partial[k]=[f]=b$. We also have $q \circ k=\left.h\right|_{Q^{\prime}}$, so $s^{\prime}(b)$ is represented by $\left.h\right|_{Q^{\prime}}$ as claimed.

To complete the proof of Lemma 14.1 we observe that $h$ and $\left.h\right|_{Q^{\prime}}$ represent the same element of $\left(\Omega_{\mathrm{IP}}\right)_{i+1}(\Sigma W, *)$ because the composite

$$
I \times Q \stackrel{p}{\rightarrow} Q \stackrel{h}{\rightarrow} \Sigma W
$$

(where $p$ is the projection) is a bordism, in the sense of [C79, Section 4], between $h$ and $\left.h\right|_{Q^{\prime}}$.

\section{Appendix A. The intrinsic filtration of a Finite-Dimensional PL SPACE}

Let $X$ be a PL space. Say that two points $x_{1}, x_{2} \in X$ are equivalent if there are neighborhoods $U_{1}$ of $x_{1}$ and $U_{2}$ of $x_{2}$ with a PL homeomorphism of pairs $\left(U_{1}, x_{1}\right) \approx\left(U_{2}, x_{2}\right)$. Let $X$ be finite dimensional. Choose a triangulation of $X$, and let $X(i)$ be the $i$-skeleton of this triangulation. The intrinsic filtration of $X$ is the filtration $X^{i}$ for which $x \in X^{i}$ if and only if all points equivalent to $x$ are in $X(i)$. This filtration is independent of the chosen 
triangulation because it is the coarsest PL CS stratification 12 of $X$ (cf. [F, Remark 2.10.7]). We will use the notation $X^{i}$ throughout this appendix to denote the intrinsic filtration of $X$.

Proposition A.1. Let $X$ be a finite-dimensional PL space.

(i) If $U$ is an open subset of $X$ then $U^{i}=X^{i} \cap U$.

(ii) If $M$ is a PL manifold of dimension $m$ then

$$
(X \times M)^{i}= \begin{cases}X^{i-m} \times M & m \leq i \leq \operatorname{dim} X+m, \\ \emptyset & \text { otherwise }\end{cases}
$$

(iii) If $f: X \rightarrow Y$ is a PL homeomorphism then $f\left(X^{i}\right)=Y^{i}$.

(iv) If $X$ is a $P L$ pseudomanifold then the intrinsic filtration on $X$ is a stratification in the sense of Definition 2.5.

Proof. Part (i) is [F, Lemma 2.10.16], part (ii) is [F, Lemma 2.10.17], part (iii) is immediate from the definition of the intrinsic filtration, and part (iv) is [F, Proposition 2.10.18].

The following fact is [F, Proposition 2.10.23].

Proposition A.2. Let $X$ be a $P L$-pseudomanifold, and define subsets $X[i]$ by letting $X[i] \cap(X-\partial X)=(X-\partial X)^{i}$ and

$$
X[i] \cap \partial X= \begin{cases}(\partial X)^{i-1} & 1 \leq i \leq \operatorname{dim} X, \\ \emptyset & \text { otherwise. }\end{cases}
$$

Then the filtration $X[i]$ gives $X$ the structure of a stratified $P L \partial$ pseudomanifold.

\section{Appendix B. Modules over AdDitive CATEGories.}

See [Lück, Section 9A] for an introduction to this topic.

An additive category is a small category in which the morphism sets are abelian groups and the composition is bilinear. A functor between additive categories is additive if it is a homomorphism on each morphism set.

Example B.1. A ring is the same thing as an additive category with a single object, and a ring homomorphism is an additive functor.

By a left (resp., right) module over an additive category $\mathcal{C}$ we mean a covariant (resp., contravariant) additive functor $\mathcal{M}$ from $\mathcal{C}$ to the category of abelian groups; we write $\mathcal{M}_{c}$ for the value of $\mathcal{M}$ at an object $c$ of $\mathcal{C}$.

If $\mathcal{M}$ and $\mathcal{N}$ are left modules over $\mathcal{C}$ we define $\operatorname{Hom}_{\mathcal{C}}(\mathcal{M}, \mathcal{N})$ to be the abelian group of natural transformations.

If $\mathcal{M}$ is a right module over $\mathcal{C}$ and $\mathcal{N}$ is a left module, the tensor product $\mathcal{M} \otimes_{\mathcal{C}} \mathcal{N}$ is the abelian group

$$
\bigoplus_{c \in \mathrm{Ob}(\mathcal{C})}\left(\mathcal{M}_{c} \otimes \mathcal{N}_{c}\right) / Q
$$

\footnotetext{
${ }^{12}$ The definition of CS stratification is a weaker version of Definition 2.5
} 
here $Q$ is generated by the elements $\mathcal{M}(f)(m) \otimes n-m \otimes \mathcal{N}(f)(n)$, where $f$ runs through the morphisms in $\mathcal{C}, m \in \mathcal{M}_{\text {target }(f)}$, and $n \in \mathcal{N}_{\text {source }(f)}$.

The tensor product of two additive categories $\mathcal{C}$ and $\mathcal{C}^{\prime}$ is the additive category whose object set is $\mathrm{Ob}(\mathcal{C}) \times \mathrm{Ob}\left(\mathcal{C}^{\prime}\right)$, and whose morphism set from $\left(c_{1}, c_{1}^{\prime}\right)$ to $\left(c_{2}, c_{2}^{\prime}\right)$ is $\operatorname{Mor}_{\mathcal{C}}\left(c_{1}, c_{2}\right) \otimes \operatorname{Mor}_{\mathcal{C}}^{\prime}\left(c_{1}^{\prime}, c_{2}^{\prime}\right)$.

A $\left(\mathcal{C}, \mathcal{C}^{\prime}\right)$ bimodule is a left module over $\mathcal{C}^{\text {op }} \otimes \mathcal{C}^{\prime}$.

Let $F: \mathcal{C} \rightarrow \mathcal{C}^{\prime}$ be an additive functor. The canonical bimodule determined by $F$ is the $\left(\mathcal{C}, \mathcal{C}^{\prime}\right)$ bimodule $\mathcal{P}_{F}$ which takes $\left(c, c^{\prime}\right)$ to $\operatorname{Mor}_{\mathcal{C}^{\prime}}\left(F(c), c^{\prime}\right)$, with the evident action on morphisms. If $\mathcal{M}$ is a left $\mathcal{C}$ module, the Kan extension of $\mathcal{M}$ along $F$, denoted $\operatorname{Kan}_{F} \mathcal{M}$, is the left $\mathcal{C}^{\prime}$ module that takes $c^{\prime}$ to $\mathcal{P}_{F}\left(-, c^{\prime}\right) \otimes_{\mathcal{C}} \mathcal{M}$, with the evident action on morphisms.

Example B.2. If $h: R \rightarrow S$ is a ring homomorphism and $M$ is an $R$-module then $\operatorname{Kan}_{h} M$ is isomorphic to $S \otimes_{R} M$.

There is a natural map $\iota: \mathcal{M} \rightarrow \operatorname{Kan}_{F} \mathcal{M}$ which takes $m \in \mathcal{M}_{c}$ to $\operatorname{id}_{F(c)} \otimes m$. There is a natural bijection between $\operatorname{Hom}_{\mathcal{C}^{\prime}}\left(\operatorname{Kan}_{F} \mathcal{M}, \mathcal{N}\right)$ (where $\mathcal{N}$ is a $\mathcal{C}^{\prime}$ module) and $\operatorname{Hom}_{\mathcal{C}}(\mathcal{M}, \mathcal{N})$ which takes $h$ to $h \circ \iota$.

If $G: \mathcal{C}^{\prime} \rightarrow \mathcal{C}^{\prime \prime}$ is an additive functor there is an isomorphism

$$
\mathcal{P}_{G} \otimes \mathcal{C}^{\prime} \mathcal{P}_{F} \cong \mathcal{P}_{G \circ F}
$$

of $\left(\mathcal{C}, \mathcal{C}^{\prime \prime}\right)$ bimodules which on objects is induced by the composition map $\operatorname{Mor}_{\mathcal{C}^{\prime \prime}}\left(G\left(c^{\prime}\right), c^{\prime \prime}\right) \otimes \operatorname{Mor}_{\mathcal{C}^{\prime}}\left(F(c), c^{\prime}\right) \rightarrow \operatorname{Mor}_{\mathcal{C}^{\prime \prime}}\left(G(F(c)), c^{\prime \prime}\right)$; thus there is an isomorphism of $\mathcal{C}^{\prime \prime}$ modules

$$
\operatorname{Kan}_{G}\left(\operatorname{Kan}_{F}(\mathcal{M})\right) \cong \operatorname{Kan}_{G \circ F} \mathcal{M} .
$$

For use in Section 10 we record

Remark B.3. Recall the definition of the additive category $\mathbb{Z}\left[\pi_{1} Z\right]$ from Section 7 (and also recall that we denote composition of path homotopy classes by letting $\delta \gamma$ be "first $\gamma$, then $\delta$ ", analogously to composition of functions). Let $g: Z \rightarrow Z^{\prime}$ be a map of connected spaces, and let $g_{*}$ : $\mathbb{Z}\left[\pi_{1} Z\right] \rightarrow \mathbb{Z}\left[\pi_{1} Z^{\prime}\right]$ be the induced functor. Let $\mathcal{M}$ be a $\mathbb{Z}\left[\pi_{1} Z\right]$ module and let $z \in Z$. We can give an explicit description of $\operatorname{Kan}_{g_{*}}(\mathcal{M})_{g(z)}$, as follows. By definition, $\operatorname{Kan}_{g_{*}}(\mathcal{M})_{g(z)}$ is $\mathcal{P}_{g_{*}}(-, g(z)) \otimes_{\mathbb{Z}\left[\pi_{1} Z\right]} \mathcal{M}$, and $\mathcal{P}_{g_{*}}(w, g(z))$ is the free abelian group generated by the path homotopy classes $\delta$ from $g(w)$ to $g(z)$. There is a map

$$
\mathbb{Z}\left[\pi_{1}\left(Z^{\prime}, g(z)\right] \otimes_{\mathbb{Z}\left[\pi_{1}(Z, z)\right]} \mathcal{M}_{z} \rightarrow \operatorname{Kan}_{g_{*}}(\mathcal{M})_{g(z)}\right.
$$

which takes $\gamma \otimes m$ to the class of $\gamma \otimes m$. This map is an isomorphism (it's straightforward to check that its inverse takes $\delta \otimes n$, where $\delta$ is a path homotopy class from $g(w)$ to $g(z)$ and $n \in \mathcal{M}_{w}$, to $\delta(g \circ \epsilon)^{-1} \otimes \epsilon n$, where $\epsilon$ 
is any path homotopy class from $w$ to $z$ ). The diagram

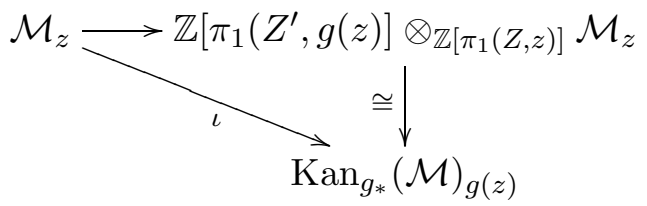

commutes.

Similarly, for $z_{1}, z_{2} \in Z$ and modules $\mathcal{M}_{1}, \mathcal{M}_{2}$ over $\mathbb{Z}\left[\pi_{1} Z\right]$, there is an isomorphism

$$
\begin{aligned}
\left(\mathbb{Z}\left[\pi_{1}\left(Z^{\prime}, g\left(z_{1}\right)\right] \otimes_{\mathbb{Z}\left[\pi_{1}\left(Z, z_{1}\right)\right]}\left(\mathcal{M}_{1}\right)_{z_{1}}\right)\right. & \otimes\left(\mathbb{Z}\left[\pi_{1}\left(Z^{\prime}, g\left(z_{2}\right)\right] \otimes_{\mathbb{Z}\left[\pi_{1}\left(Z, z_{2}\right)\right]}\left(\mathcal{M}_{2}\right)_{z_{2}}\right)\right. \\
& \rightarrow \operatorname{Kan}_{g_{*} \otimes g_{*}}\left(\mathcal{M}_{1} \otimes \mathcal{M}_{2}\right)_{\left(g\left(z_{1}\right), g\left(z_{2}\right)\right)} .
\end{aligned}
$$

\section{Appendix C. Subdivision of Singular simplices}

In this section we record some facts related to subdivision which will be needed in the next section.

Recall that intersection chains can be defined for any filtered space ( F03, Section 2]). For a chain $\eta$, write $\operatorname{supp}(\eta)$ for the support of $\eta$, that is, the union of the images of the singular simplices that have nonzero coefficient in $\eta$.

Proposition C.1. Let $Z$ be a filtered space, let $U \subset Z$ be open, and let $\bar{p}$ be a perversity. Then there is an operation which takes each singular simplex $s$ to a chain $\bar{s}$, with the following properties.

(i) If $\operatorname{supp}(s) \subset U$ then $\bar{s}=s$.

(ii) $\operatorname{supp}(\bar{s}) \subset U \cap \operatorname{supp}(s)$.

(iii) If $s$ is allowable then so are all singular simplices that belong to $\bar{s}$.

(iv) If $\xi=\sum a_{i} s_{i}$ is an element of $I S_{*}^{\bar{p}}(Z ; \mathbb{Z})$ with $a_{i} \in \mathbb{Z}$, write

$$
\bar{\xi}=\sum a_{i} \bar{s}_{i}
$$

Then $\bar{\xi} \in I S_{*}^{\bar{p}}(U ; \mathbb{Z})$.

Proof. In the construction of [FM13b, page 155 line -7 to page 156 line 19], ignore $\xi_{i}$, replace $\mathfrak{B}$ by the set of all singular simplices, replace $\mathfrak{B}^{j}$ by the set of singular simplices of dimension $j$, and replace $U_{1}$ by $U$. Define $\bar{s}$ by the equation on line 15 of page 156 of [FM13b]. Then (i) and (ii) are immediate, and (iii) follows from [F07, Lemma 2.6]. To see (iv), first note that Equation (9) on page 156 of [FM13b] remains valid with the same proof. Let $k$ be the dimension of $\xi$. Then

$$
\partial \bar{\xi} \equiv \sum_{t \in \mathfrak{B}^{k-1}}\left[\sum_{\text {notallowable }} c_{s \in \mathfrak{B}^{k}}(s) c_{\partial s}(t)\right] \bar{t}
$$

modulo allowable singular simplices. The expression in brackets is equal to the coefficient of $t$ in $\partial \xi$, and this is zero because $\xi$ is an intersection chain. Hence $\bar{\xi}$ is allowable. 
Lemma C.2. Let $Z$ be a filtered space, let $\left\{W_{1}, \ldots, W_{n}\right\}$ be an open cover of $Z$, let $\bar{p}$ be a perversity and let $i \in \mathbb{Z}$. Then

(i) the intersection of $I S_{i}^{\bar{p}}(Z ; \mathbb{Z})$ with $\sum_{j} S_{i}\left(W_{j} ; \mathbb{Z}\right)$ (considered as subgroups of $\left.S_{i}(Z ; \mathbb{Z})\right)$ is $\sum_{j} I S_{i}^{\bar{p}}\left(W_{j} ; \mathbb{Z}\right)$, and

(ii) $I S_{i}^{\bar{p}}(Z ; \mathbb{Z}) / \sum_{j} I S_{i}^{\bar{p}}\left(W_{j} ; \mathbb{Z}\right)$ is free over $\mathbb{Z}$.

Proof of Lemma C.2. For part (i), the proof is by induction on $n$. Let

$$
\xi \in I S_{i}^{\bar{p}}(Z ; \mathbb{Z}) \cap \sum_{j=1}^{n} S_{i}\left(W_{j} ; \mathbb{Z}\right) .
$$

Write

$$
\xi=\sum a_{m} s_{m}
$$

then every $\operatorname{supp}\left(s_{m}\right)$ is contained in some $W_{j}$. Apply Proposition C.1 with $U=W_{1}$ to get $\bar{\xi} \in I S_{*}^{\bar{p}}\left(W_{1} ; \mathbb{Z}\right)$. Parts (i) and (ii) of Proposition C.1 show that every singular simplex that belongs to $\xi-\bar{\xi}$ has support in some $W_{j}$ with $j \geq 2$. Let $Z^{\prime}=\cup_{j=2}^{n} W_{j}$. Then $\xi-\bar{\xi}$ is an element of

$$
I S_{i}^{\bar{p}}\left(Z^{\prime} ; \mathbb{Z}\right) \cap \sum_{j=2}^{n} S_{i}\left(W_{j} ; \mathbb{Z}\right),
$$

so by the inductive hypothesis

$$
\xi-\bar{\xi} \in \sum_{j=2}^{n} I S_{i}^{\bar{p}}\left(W_{j} ; \mathbb{Z}\right),
$$

and therefore

$$
\xi \in \sum_{j=1}^{n} I S_{i}^{\bar{p}}\left(W_{j} ; \mathbb{Z}\right)
$$

as required.

For part (ii), note that by part (i) the map

$$
I S_{i}^{\bar{p}}(Z ; \mathbb{Z}) / \sum_{j} I S_{i}^{\bar{p}}\left(W_{j} ; \mathbb{Z}\right) \rightarrow S_{i}(Z, \mathbb{Z}) / \sum_{j} S_{i}\left(W_{j} ; \mathbb{Z}\right)
$$

is 1-1 and that $S_{i}(Z, \mathbb{Z}) / \sum_{j} S_{i}\left(W_{j} ; \mathbb{Z}\right)$ is freely generated by the singular simplices that do not land in any $W_{j}$.

Remark C.3. Part (i) of this result gives a simple proof of [FM13b, Lemma 6.11], as follows. With the notation of that Lemma,

$$
\xi_{1}=-\xi_{2}-\cdots-\xi_{m},
$$

so every singular simplex that belongs to $\xi_{1}$ has support in some $U_{i}$ with $i \geq 2$. Thus

$$
\xi_{1} \in I S_{*}^{\bar{p}}(X ; \mathbb{Z}) \cap \sum_{i=2}^{m} S_{*}\left(U_{1} \cap U_{i} ; \mathbb{Z}\right),
$$


and by Lemma $\underline{\text { C.2 }}(\mathrm{i})$ there exist $\eta_{i} \in I S_{*}^{\bar{p}}\left(U_{i} \cap U_{1} ; \mathbb{Z}\right)$ for $2 \leq i \leq m$ with

$$
\xi_{1}=-\eta_{2}-\cdots-\eta_{m}
$$

as required.

\section{Appendix D. Extensions of some Results from [FM13b]}

In this appendix we show that the results of FM13b, Subsections 6.2 and $6.3]$ remain valid with the field $F$ replaced by $\mathbb{Z}$. We will use the notation of those subsections, except that we denote singular chains by $S_{*}$ instead of $C_{*}$ and we write $I S_{*}^{\bar{p}}$ instead of $I^{\bar{p}} C_{*}$ and $I S_{*}^{\bar{p}, \mathcal{U}}$ instead of $I_{\mathcal{U}}^{\bar{p}} C_{*}$.

First we have an analog of [FM13b, Lemma 6.6] (the extra generality in the statement is used in Subsections 6.3 and 10.11).

We need some definitions and notation. Let $Z$ be a filtered space and let $\bar{p}$ be a perversity. Let $p: \tilde{Z} \rightarrow Z$ be a regular cover with automorphism group $\pi$, and for a subset $A \subset Z$ write $\tilde{A}$ for $p^{-1}(A)$. Recall that $A$ is said to be evenly covered if the cover $\tilde{A} \rightarrow A$ is trivial. Let $\mathcal{S}$ be a finite collection of subsets of $Z$, and let $\mathcal{C}(\mathcal{S})$ be the category whose objects are the elements of $\mathcal{S}$ and whose morphisms are inclusions. Let

$$
\Phi: \mathcal{C}(\mathcal{S}) \rightarrow \mathbb{Z}[\pi]-\bmod
$$

be the functor that takes $A$ to $I S_{*}^{\bar{p}}(\tilde{A} ; \mathbb{Z})$. For each element $A$ of $S$ let $\mathcal{C}(A)$ be the full subcategory of $\mathcal{C}(\mathcal{S})$ whose objects are the elements of $\mathcal{S}$ which are proper subsets of $A$, and let $\mathbf{C}(A)$ be the cokernel of the map

$$
\underset{B \in \mathcal{C}(A)}{\operatorname{colim}} I S_{*}^{\bar{p}}(B ; \mathbb{Z}) \rightarrow I S_{*}^{\bar{p}}(A ; \mathbb{Z})
$$

Let

$$
\Psi: \mathcal{C}(\mathcal{S}) \rightarrow \mathbb{Z}[\pi]-\bmod
$$

be the functor which takes $A$ to

$$
\bigoplus_{B \in \mathcal{S} \text { and } B \subset A} \mathbb{Z}[\pi] \otimes \mathbf{C}(B)
$$

and has the obvious definition on morphisms.

Lemma D.1. Suppose that, for every $A \in \mathcal{S}$,

(a) A is evenly covered,

(b) the map

$$
\operatorname{colim}_{B \in \mathcal{C}(A)} I S_{*}^{\bar{p}}(B ; \mathbb{Z}) \rightarrow I S_{*}^{\bar{p}}(A ; \mathbb{Z})
$$

is a monomorphism, and

(c) $\mathbf{C}(A)$ is free over $\mathbb{Z}$.

Then

(i) $\Phi$ is naturally isomorphic to $\Psi$, and

(ii) $\operatorname{colim}_{A \in \mathcal{C}(\mathcal{S})} I S_{*}^{\bar{p}}(\tilde{A} ; \mathbb{Z}) \cong \bigoplus_{A \in \mathcal{S}} \mathbb{Z}[\pi] \otimes \mathbf{C}(A)$, and

(iii) for each $\mathcal{S}^{\prime} \subset \mathcal{S}$, the map

$$
\operatorname{colim}_{A \in \mathcal{C}\left(\mathcal{S}^{\prime}\right)} I S_{*}^{\bar{p}}(\tilde{A} ; \mathbb{Z}) \rightarrow \operatorname{colim}_{A \in \mathcal{C}(\mathcal{S})} I S_{*}^{\bar{p}}(\tilde{A} ; \mathbb{Z})
$$


has a left inverse over $\mathbb{Z}[\pi]$.

Proof of Lemma D.1. Part (ii) follows from part (i), since $\bigoplus_{A \in \mathcal{S}} \mathbb{Z}[\pi] \otimes \mathbf{C}(A)$ satisfies the universal property to be $\operatorname{colim}_{A \in \mathcal{C}(\mathcal{S})} \Psi(A)$, and (iii) is immediate from part (ii).

Proof of (i). For each $A \in \mathcal{S}$, choose an isomorphism $\iota_{A}$ from the trivial cover $\pi \times A \rightarrow A$ to $\left.p\right|_{A}$ and a splitting $s_{A}$ of the map $I S_{*}^{\bar{p}}(A ; \mathbb{Z}) \rightarrow \mathbf{C}(A)$. Let

$$
\nu_{A}: \Psi(A) \rightarrow \Phi(A)
$$

be the map whose restriction to $\mathbb{Z}[\pi] \otimes \mathbf{C}(B)$ is the composite

$\mathbb{Z}[\pi] \otimes \mathbf{C}(B) \stackrel{1 \otimes s_{B}}{\longrightarrow} \mathbb{Z}[\pi] \otimes I S_{*}^{\bar{p}}(B ; \mathbb{Z}) \cong I S_{*}^{\bar{p}}(\pi \times B ; \mathbb{Z}) \stackrel{\iota_{B}}{\longrightarrow} I S_{*}^{\bar{p}}(\tilde{B} ; \mathbb{Z}) \rightarrow I S_{*}^{\bar{p}}(\tilde{A} ; \mathbb{Z})$.

This is a natural transformation $\Psi \rightarrow \Phi$. To see that $\nu_{A}$ is an isomorphism, let us assume inductively that this has been shown for all $B \subset A$. Consider the diagram

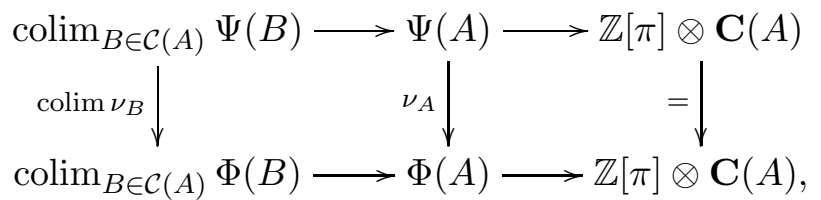

where the upper right arrow is the projection and the lower right arrow is the composite

$$
\Phi(A) \stackrel{\iota_{A}^{-1}}{\longrightarrow} I S_{*}^{\bar{p}}(\pi \times A ; \mathbb{Z}) \cong \mathbb{Z}[\pi] \otimes I S_{*}^{\bar{p}}(A ; \mathbb{Z}) \rightarrow \mathbb{Z}[\pi] \otimes \mathbf{C}(A) .
$$

The diagram commutes, and the left vertical arrow is an isomorphism, so it suffices to show that the rows are short exact. For the upper row this is clear, and for the lower row this follows from hypothesis (b) and the definition of $\mathbf{C}(A)$.

Remark D.2. If all $A \in \mathcal{S}$ are open and $\mathcal{S}$ is closed under intersection then hypotheses (a) and (b) of Lemma D.1 are satisfied: (a) follows from FM13b, Proposition 6.3] and (b) from [FM13b, Proposition 6.3] and Lemma C.2(ii).

Next we have the analog of [FM13b, Proposition 6.4].

Proposition D.3. Let $Z$ be a filtered space and let $A$ be an open subset of $Z$. Let $\bar{p}$ be a perversity.

(i) If $Z$ has a finite covering by evenly covered open sets (in particular, if $Z$ is compact) then $I S_{*}^{\bar{p}}(\tilde{Z}, \tilde{A} ; \mathbb{Z})$ is chain homotopy equivalent over $\mathbb{Z}[\pi]$ to a nonnegatively-graded chain complex of free $\mathbb{Z}[\pi]$-modules.

(ii) For all $Z, I S_{*}^{\bar{p}}(\tilde{Z}, \tilde{A} ; \mathbb{Z})$ is chain homotopy equivalent over $\mathbb{Z}[\pi]$ to a nonnegatively-graded chain complex of flat $\mathbb{Z}[\pi]$-modules.

This follows from the proof of [FM13b, Proposition 6.4], using Lemma D.1 and Remark D.2(ii) instead of [FM13b, Lemma 6.6].

Our next goal is to prove an analogue of [FM13b, Proposition 6.5]. First recall that with $\mathbb{Z}$ coefficients the Künneth theorem that was used in the 
proof of [FM13b, Proposition 6.5] requires a hypothesis about torsion; see [F, Definition 6.4.5 and Theorem 6.4.7] for the precise statement. It will be useful to have the following terminology from GS83.

Definition D.4. Let $\bar{p}$ be any perversity. A pseudomanifold is called $l o-$ cally $\bar{p}$-torsion free if $I H_{c-2-\bar{p}(c)}^{\bar{p}}\left(L^{\prime}\right)$ is torsion free for every link $L^{\prime}$, where $\operatorname{dim} L^{\prime}=c-1$.

Remark D.5. IP-spaces are locally $\bar{m}$-torsion free, since if $c-1$ is even, $c-2-\bar{m}(c)=\frac{1}{2}(c-1)$ and $I H_{(c-1) / 2}\left(L^{\prime}\right)=0$, while if $c-1$ is odd, $c-2-\bar{m}(c)=\frac{c}{2}-1$ and $I H_{c / 2-1}\left(L^{\prime}\right)$ is torsion free.

The proof of [FM13b, Proposition 6.5] goes through without change to show

Proposition D.6. Let $X$ be a stratified PL $\partial$-pseudomanifold which is locally $\bar{p}$-torsion free or locally $\bar{q}$-torsion free and let $A$ be an open subset of $X$. Then the cross product

$$
I S_{*}^{\bar{p}}(\tilde{X}, \tilde{A} ; \mathbb{Z}) \otimes_{\mathbb{Z}} I S_{*}^{\bar{q}}(\tilde{X}, \tilde{A} ; \mathbb{Z}) \rightarrow I S_{*}^{Q_{\bar{p}}, \bar{q}}(\tilde{X} \times \tilde{X}, \tilde{A} \times \tilde{X} \cup \tilde{X} \times \tilde{A} ; \mathbb{Z})
$$

induces a quasi-isomorphism

$$
\begin{aligned}
\mathbb{Z} \otimes_{\mathbb{Z}[\pi]}\left(I S_{*}^{\bar{p}}(\tilde{X}, \tilde{A} ; \mathbb{Z}) \otimes_{\mathbb{Z}}\right. & \left.I S_{*}^{\bar{q}}(\tilde{X}, \tilde{A} ; \mathbb{Z})\right) \\
& \rightarrow \mathbb{Z} \otimes_{\mathbb{Z}[\pi]} I S_{*}^{Q_{\bar{p}}, \bar{q}}(\tilde{X} \times \tilde{X}, \tilde{A} \times \tilde{X} \cup \tilde{X} \times \tilde{A} ; \mathbb{Z}) .
\end{aligned}
$$

Remark D.7. Let us say that a perversity is classical if it satisfies the original definition given by Goresky and MacPherson in GM80]. By [F, Theorem 5.5.1], if $X$ is a stratified PL pseudomanifold, $\mathfrak{X}$ is the same PL pseudomanifold with the intrinsic stratification, and $\bar{p}$ is a classical perversity, then the canonical map

$$
I S_{*}^{\bar{p}}(X ; \mathbb{Z}) \rightarrow I S_{*}^{\bar{p}}(\mathfrak{X} ; \mathbb{Z})
$$

is a quasi-isomorphism.

Next we have the analogue of [FM13b, Proposition 5.15], except that we require the perversity to be classical.

Proposition D.8. Let $X$ be a compact stratified PL $\partial$-pseudomanifold. Let $\bar{p}$ be a classical perversity. Then $I S_{*}^{\bar{p}}(\tilde{X} ; \mathbb{Z})$ is quasi-isomorphic over $\mathbb{Z}[\pi]$ to a finite $\mathbb{Z}[\pi]$ chain complex.

Proof. It suffices to show that [FM13b, Lemma 6.7] remains valid with $F$ replaced by $\mathbb{Z}$. The only place in the proof of that result where the hypothesis that $F$ is a field is used is in the second paragraph of the proof of Lemma 6.9 , as part of the verification that $I S_{*}^{\bar{p}}(\operatorname{St}(s) ; F)$ is homotopy finite over $F$, so we need to prove that $I S_{*}^{\bar{p}}(\operatorname{St}(s) ; \mathbb{Z})$ is homotopy finite over $\mathbb{Z}$, where $s$ is a simplex with no vertices in $\partial X$.

For the remainder of the proof, for a PL space $Y$, we will write $Y^{*}$ for $Y$ with its intrinsic filtration. 
It suffices to show that $I S_{*}^{\bar{p}}\left(\operatorname{St}(s)^{*} ; \mathbb{Z}\right)$ is homotopy finite over $\mathbb{Z}$, because this is quasi-isomorphic to $I S_{*}^{\bar{p}}(\operatorname{St}(s) ; \mathbb{Z})$ by Remark D.7, and hence it is chain homotopy equivalent because both chain complexes are free over $\mathbb{Z}$.

It is shown in the proof of [FM13b, Lemma 6.9] that $\operatorname{St}(s)$ is homeomorphic as a PL space to the open cone $c^{\circ} A$, where $A=(\partial s) * \operatorname{Lk}(s)$, so it suffices to show that $I S_{*}^{\bar{p}}\left(\left(c^{\circ} A\right)^{*} ; \mathbb{Z}\right)$ is homotopy finite over $\mathbb{Z}$. Now by Proposition A.1 the restriction of the filtration of $\left(c^{\circ} A\right)^{*}$ to $A \times(0,1)$ is the same as the filtration of $A^{*} \times(0,1)$. Hence if we let let $B$ be

$$
\left(\left[0, \frac{1}{2}\right] \times A\right) /(0 \times x \sim 0 \times y),
$$

with the filtration inherited from $\left(c^{\circ} A\right)^{*}$, then the evident homotopy equivalence from $\left(c^{\circ} A\right)^{*}$ to $B$ is a stratified homotopy equivalence (see [FM13a, Appendix A] for the definition of stratified homotopy equivalence). Thus (by the last paragraph of [FM13a, Appendix A]) it suffices to show that the intersection chain complex of $B$ with this filtration is homotopy finite over $\mathbb{Z}$.

Next we need a lemma:

Lemma D.9. $B$, with the filtration inherited from $\left(c^{\circ} A\right)^{*}$, is a stratified $P L$ D-pseumanifold.

Before proving this, we note that it implies, by [F, Corollary 5.4.6], that the intersection chain complex of $B$ is quasi-isomorphic (and hence chain homotopy equivalent) to the simplicial intersection chain complex $I C_{*}^{\bar{p}, T}(B ; \mathbb{Z})$ for any suitable triangulation $T$. Since $B$ is compact, $I C_{*}^{\bar{p}, T}(B ; \mathbb{Z})$ is a finite chain complex, which completes the proof of Proposition D.8.

Proof of Lemma D.9. First we show that $\operatorname{Lk}(s)$ is a PL pseudomanifold. The fact that it satisfies part (a) of Definition 2.1 is immediate from the corresponding condition for $X$ and the definition of $\operatorname{Lk}(s)$. The fact that $\operatorname{Lk}(s)$ satisfies part (b) of Definition 2.1 follows easily from the corresponding condition for $X$, the definition of $\operatorname{Lk}(s)$, and the fact that $s$ is not contained in $\partial X$.

Now it follows that $A=(\partial s) * \operatorname{Lk}(s)$ is a PL pseudomanifold, and hence $A^{*}$ is a stratified PL pseudomanifold by Proposition A.1(iv).

Next we observe that $B$ is the union of two open sets $U_{1}$ and $U_{2}$, each of which (with its inherited filtration) is a $\partial$-stratified PL pseudomanifold: let $U_{1}=\left(\left[0, \frac{1}{2}\right) \times A\right) /(0 \times x \sim 0 \times y)$, which is a stratified PL pseudomanifold because it is homeomorphic as a filtered space to $\left(c^{\circ} A\right)^{*}$ and hence to $\operatorname{St}(s)^{*}$, and let $U_{2}$ be $\left(\left(0, \frac{1}{2}\right] \times A\right)$, which is a stratified PL $\partial$-pseudomanifold because (by Proposition A.1) it is homeomorphic as a filtered space to $\left(\left(0, \frac{1}{2}\right] \times\right.$ $\left.A^{*}\right)$.

\section{Appendix E. Universal Poincaré and Lefschetz DUality}

In this appendix we give the analogues of [FM13b, Theorems 4.1 and 4.5] for $\mathbb{Z}$ coefficients. 
First we construct a suitable cap product, following the method of FM13b, Section 3].

Let $X$ be a stratified PL $\partial$-pseudomanifold.

Let $p: \tilde{X} \rightarrow X$ be a regular cover with group $\pi$. For any subset $A$ of $X$ we write $\tilde{A}$ for $p^{-1}(A)$. We assume that $\tilde{X}$ is stratified by the preimages of the strata of $X$. Note that $I S_{*}^{\bar{p}}(\tilde{X} ; \mathbb{Z})$ possesses a left $\mathbb{Z}[\pi]$-module structure induced by the geometric action of $\pi$ on $\tilde{X}$.

Notation E.1. (i) Given a perversity $\bar{p}$ on $X$, the perversity on $\tilde{X}$ which takes a stratum $S$ to $\bar{p}(p(S))$ will also be denoted by $\bar{p}$.

(ii) We will write $I \bar{S}_{\bar{p}}^{*}(\tilde{X} ; \mathbb{Z})$ for $\operatorname{Hom}_{\mathbb{Z}[\pi]}\left(I S_{*}^{\bar{p}}(\tilde{X} ; \mathbb{Z}), \mathbb{Z}[\pi]\right)$ and $I \bar{H}_{\bar{p}}^{*}(\tilde{X} ; \mathbb{Z})$ for the cohomology groups of this complex.

Now recall Definition D.4 and suppose that $\bar{p}, \bar{q}$ and $\bar{r}$ are perversities with $D \bar{r} \geq D \bar{p}+D \bar{q}$ and that $X$ is either locally $\bar{p}$-torsion free or locally $\bar{q}$-torsion free.

Let

$$
\tilde{d}: I H_{*}^{\bar{r}}(X ; \mathbb{Z}) \rightarrow H_{*}\left(I S_{*}^{\bar{p}}(\tilde{X} ; \mathbb{Z})^{t} \otimes_{\mathbb{Z}[\pi]} I S_{*}^{\bar{q}}(\tilde{X} ; \mathbb{Z})\right)
$$

be the composition

$$
\begin{aligned}
I H_{*}^{\bar{r}}(X ; \mathbb{Z}) & \cong H_{*}\left(\mathbb{Z} \otimes_{\mathbb{Z}[\pi]} I S_{*}^{\bar{r}}(\tilde{X} ; \mathbb{Z})\right) \\
& \stackrel{1 \otimes d}{\longrightarrow} H_{*}\left(\mathbb{Z} \otimes_{\mathbb{Z}[\pi]} I S_{*}^{Q_{\bar{p}}, \bar{q}}(\tilde{X} \times \tilde{X} ; \mathbb{Z})\right) \\
& \cong H_{*}\left(\mathbb{Z} \otimes_{\mathbb{Z}[\pi]}\left(I S_{*}^{\bar{p}}(\tilde{X} ; \mathbb{Z}) \otimes_{\mathbb{Z}} I S_{*}^{\bar{q}}(\tilde{X} ; \mathbb{Z})\right)\right) \\
& \cong H_{*}\left(I S_{*}^{\bar{p}}(\tilde{X} ; \mathbb{Z})^{t} \otimes_{\mathbb{Z}[\pi]} I S_{*}^{\bar{q}}(\tilde{X} ; \mathbb{Z})\right) .
\end{aligned}
$$

Here $d$ is the diagonal map given by [FM13a, Proposition 4.2.1], the first isomorphism is given by Proposition [FM13b, Proposition 6.1.3], and the second isomorphism is given by Proposition D.6 (this is why the torsion free assumption is needed). The third isomorphism is elementary.

Now we can define the cap product

$$
I \bar{H}_{\bar{q}}^{i}(\tilde{X} ; \mathbb{Z}) \otimes I H_{j}^{\bar{r}}(X ; \mathbb{Z}) \rightarrow I H_{j-i}^{\bar{p}}(\tilde{X}, ; \mathbb{Z})
$$

by

$$
\alpha \frown x=(1 \otimes \alpha) \tilde{d}(x)
$$

(using the fact that $H_{*}\left(I S_{*}^{\bar{p}}(\tilde{X} ; \mathbb{Z})^{t}\right)$ is the same $\mathbb{Z}$-module as $I H_{*}^{\bar{p}}(\tilde{X} ; \mathbb{Z})$ ).

Similarly, we get a cap product

$$
I \bar{H}_{\bar{q}}^{i}(\tilde{X}, \tilde{A} ; \mathbb{Z}) \otimes I H_{j}^{\bar{r}}(X, A \cup B ; \mathbb{Z}) \rightarrow I H_{j-i}^{\bar{p}}(\tilde{X}, \tilde{B} ; \mathbb{Z})
$$

when $A$ and $B$ are open subsets of $X$.

Remark E.2. If the covering $p: \tilde{X} \rightarrow X$ is trivial then $I \bar{S}_{\bar{p}}^{*}(\tilde{X} ; \mathbb{Z})$ is canonically isomorphic to $\operatorname{Hom}\left(I S_{*}^{\bar{p}}(X ; \mathbb{Z}), \mathbb{Z}[\pi]\right)$ and hence $I \bar{H}_{\bar{p}}^{*}(\tilde{X} ; \mathbb{Z})$ is canonically isomorphic to $I H_{*}^{\bar{p}}(X ; \mathbb{Z}[\pi])$. Under this isomorphism the cap product (E.1) corresponds to the cap product

$$
I \bar{H}_{\bar{q}}^{i}(X ; \mathbb{Z}[\pi]) \otimes I H_{j}^{\bar{r}}(X ; \mathbb{Z}) \rightarrow I H_{j-i}^{\bar{p}}(X, ; \mathbb{Z}[\pi]) .
$$


Let us write

$$
\left(I \bar{H}_{\bar{p}}^{i}\right)_{c}(\tilde{X} ; \mathbb{Z})
$$

for

$$
\operatorname{colim}_{K} I \bar{H}_{\bar{p}}^{i}(\tilde{X}, \tilde{X}-\tilde{K} ; \mathbb{Z})
$$

where $K$ runs through the compact subsets of $X$ (the subscript $c$ stands for "compact supports"; cf. [F, Section 7.4]).

As in [FM13b, Section 4], we obtain a map

$$
\mathcal{D}:\left(I \bar{H}_{\bar{p}}^{i}\right)_{c}(\tilde{X} ; \mathbb{Z}) \rightarrow I H_{n-i}^{D \bar{p}}(\tilde{X} ; \mathbb{Z})
$$

Now we can state the analogue of [FM13b, Theorem 4.1] for $\mathbb{Z}$ coefficients.

Theorem E.3 (Universal Poincaré duality). Let $X$ be a $\mathbb{Z}$-oriented $P L$ pseudomanifold and let $p: \tilde{X} \rightarrow X$ be a regular covering of $X$. Suppose that $X$ is locally $\bar{p}$-torsion free (in particular this is the case if $X$ is an IP space and $\bar{p}=\bar{m}$, by Remark D.5). Then $\mathcal{D}$ is an isomorphism.

For the proof of Theorem E.3 we use [F, Theorem 5.1.4], with $F_{*}(U)=$ $\left(I \bar{H}_{\bar{p}}^{*}\right)_{c}(\tilde{U} ; \mathbb{Z}), G_{*}(U)=I H_{n-i}^{D \bar{p}}(\tilde{U} ; \mathbb{Z})$, and $\Phi=\mathcal{D}$. We need to verify the four conditions in [F, Theorem 5.1.4].

For condition 1, we first observe that the Mayer-Vietoris sequence for $F_{*}$ exists because if $U \subset V$ are open subsets of $X$ then the inclusion $I S_{*}^{\bar{p}}(\tilde{U} ; F) \hookrightarrow I S_{*}^{\bar{p}}(\tilde{V} ; F)$ is split as a map of $\mathbb{Z}[\pi]$-modules (this follows from the proof of [F07, Proposition 2.9]: use the construction in that proof with $X$ taken to be $V$ and the ordered open cover taken to be $(U, V))$. The rest of the verification of condition 1 is the same as the proof of $[\mathrm{F}$, Lemma 7.4.8], except that we use the following instead of [F, Proposition 7.3.59]:

Lemma E.4. There exist chains

$$
\begin{gathered}
\beta_{U-L} \in I S_{*}^{\bar{p}}(U-L) \otimes_{\mathbb{Z}[\pi]} I S_{*}^{\bar{q}}(U-L, U-K \cup L), \\
\beta_{U \cap V} \in I S_{*}^{\bar{p}}(U \cap V) \otimes_{\mathbb{Z}[\pi]} I S_{*}^{\bar{q}}(U \cap V, U \cap V-K \cup L)
\end{gathered}
$$

and

$$
\beta_{V-K} \in I S_{*}^{\bar{p}}(V-K) \otimes_{\mathbb{Z}[\pi]} I S_{*}^{\bar{q}}(V-K, V-K \cup L)
$$

such that $\beta_{U-L}+\beta_{U \cap V}+\beta_{V-K}$ represents $\tilde{d}\left(\Gamma_{K \cup L}\right) \in H_{*}\left(I S_{*}^{\bar{p}}(X) \otimes_{\mathbb{Z}[\pi]}\right.$ $\left.I S_{*}^{\bar{q}}(X, X-K \cup L)\right)$.

The proof of Lemma E.4 is entirely parallel to the proof of FM13a, Lemma 6.9], except that we use [FM13b, Proposition 6.1.2] to show that the map

$$
\begin{aligned}
\lambda: H_{*}\left(\mathbb{Z} \otimes_{\mathbb{Z}[\pi]} \operatorname{colim}_{W \in \mathcal{C}} I S_{*}^{Q_{\bar{p}, \bar{q}}}(\tilde{W} \times \tilde{W}, \tilde{W} \times(\tilde{W}-\tilde{K} \cup \tilde{L}))\right) \\
\rightarrow H_{*}\left(\mathbb{Z} \otimes_{\mathbb{Z}[\pi]} I S_{*}^{Q_{\bar{p}, \bar{q}}}(\tilde{Y}, \tilde{Y}-(\tilde{X} \times(\tilde{K} \cup \tilde{L})))\right)
\end{aligned}
$$

is an isomorphism.

Returning to the proof of TheoremE.3, the verification for condition 2 is the same as the corresponding part of the proof of [F, Theorem 8.2.4]; the 
excision needed for part of the argument is given by [FM13b, Proposition 6.1.2].

As background for conditions 3 and 4, we note that [F, Theorem 8.2.4] remains valid with $\mathbb{Z}[\pi]$ coefficients instead of $\mathbb{Z}$ coefficients, with the same proof.

Now for condition 3 , we observe that the covering $p$ pulls back trivially to $\mathbb{R}^{i} \times c L$, so condition 3 follows from Remark E.2 and the $\mathbb{Z}[\pi]$ version of $[\mathrm{F}$, Theorem 8.2.4] (the hypothesis of condition 3 isn't needed for this purpose).

The verification of condtion 4 is the same as for condition 3 . This concludes the proof of Theorem E.3.

Finally, we have the analog of [FM13b, Theorem 4.5].

Theorem E.5 (Universal Lefschetz Duality). Let $X$ be an $n$-dimensional compact $P L \partial$-pseudomanifold with a $\mathbb{Z}$-orientation of $X-\partial X$ and let $p$ : $\tilde{X} \rightarrow X$ be a regular covering of $X$. Suppose that $X$ is locally $\bar{p}$-torsion free (in particular this is the case if $X$ is an IP space and $\bar{p}=\bar{m}$, by Remark D.5). Then the cap product with $\Gamma_{X}$ gives isomorphisms

$$
I \bar{H}_{\bar{p}}^{i}\left(\tilde{X}, p^{-1}(\partial X) ; F\right) \rightarrow I H_{n-i}^{D \bar{p}}(\tilde{X} ; F)
$$

and

$$
I \bar{H}_{\bar{p}}^{i}(\tilde{X} ; F) \rightarrow I H_{n-i}^{D \bar{p}}\left(\tilde{X}, p^{-1}(\partial X) ; F\right) .
$$

The proof is the same as for [FM13b, Theorem 4.5].

\section{Appendix F. MUltiplicativity OF THE ASSEMBLY MAP}

This appendix gives the proof of

Proposition F.1. Let $\mathbf{F}$ be a homotopy invariant functor from spaces to spectra, and suppose that there is a natural transformation

$$
\mu: \mathbf{F}(X) \wedge \mathbf{F}(Y) \rightarrow \mathbf{F}(X \times Y) .
$$

Then the diagram

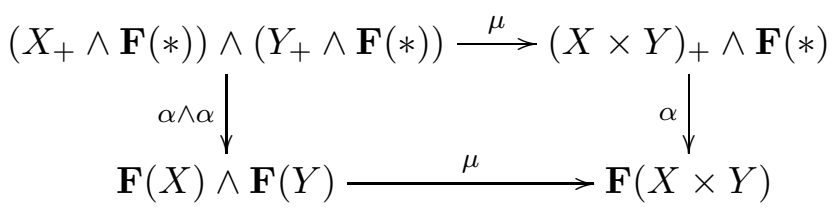

commutes up to homotopy, where $\alpha$ denotes the assembly map.

Let us recall the definition of the assembly map from [WW95, page 334]. The construction uses homotopy colimits (see [WW95, Section 1] for a brief description of the homotopy colimit construction). For a space $X$, let $\mathcal{C}_{X}$ (which is denoted $\operatorname{simp}(X)$ in [WW95]) be the category whose objects are 
maps $\Delta^{n} \rightarrow X$, and whose morphisms are commutative triangles

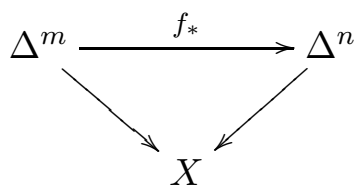

where $f_{*}$ is the map induced by a monotone injection 13 from $\{0, \ldots, m\}$ to $\{0, \ldots, n\}$. There is a natural equivalence in the homotopy category

$$
\lambda: X \rightarrow \underset{\mathcal{C}_{X}}{\operatorname{hocolim}} *
$$

(see below) where $*$ denotes the functor which takes all objects to a point. Let $D$ be the functor from $\mathcal{C}_{X}$ to spaces which takes $\Delta^{n} \rightarrow X$ to $\Delta^{n}$. The assembly map is the following composite in the homotopy category of spectra (where $\wedge$ is the derived smash product)

$$
\begin{aligned}
&\left.X_{+} \wedge \mathbf{F}(*) \stackrel{\lambda \wedge 1}{\longrightarrow} \underset{\mathcal{C}_{X}}{\operatorname{hocolim}} *\right)_{+} \wedge \mathbf{F}(*) \cong \underset{\mathcal{C}_{X}}{\operatorname{hocolim}} \mathbf{F}(*) \\
& \simeq \underset{\mathcal{C}_{X}}{\operatorname{hocolim}} \mathbf{F} \circ D \rightarrow F(X) .
\end{aligned}
$$

Our first task is to give an explicit description of $\lambda$ (this was left as an exercise for the reader in [WW95]). We need a lemma.

Lemma F.2. The map

$$
\underset{\mathcal{C}_{X}}{\operatorname{hocolim}} D \rightarrow \underset{\mathcal{C}_{X}}{\operatorname{colim}} D
$$

is a weak equivalence.

Proof. The category $\mathcal{C}_{X}$ is a Reedy category ([H03, Def 15.1.2]; the subcategory $\overrightarrow{\mathcal{C}_{X}}$ is equal to $\mathcal{C}_{X}$ and the subcategory $\overleftarrow{\mathcal{C}_{X}}$ has only identity morphisms) which has fibrant constants ([H03, Definition 15.10.1]) by the proof of [H03, Proposition 15.10.4(1)]. The functor $D$ is Reedy cofibrant ([H03, Definition 15.3.3(2)] ) and the result follows by [H03, Theorem 19.9.1(1)].

Now let $S X$ be the semisimplicial set whose $n$-simplices are the maps $\Delta^{n} \rightarrow X$. Then $\lambda$ can be chosen 14 to be the composite

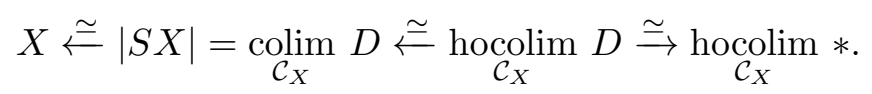

Our next lemma gives a multiplicative property of $\lambda$. Let

$$
d: \mathcal{C}_{X \times Y} \rightarrow \mathcal{C}_{X} \times \mathcal{C}_{Y}
$$

\footnotetext{
${ }^{13}$ It is not clear why WW95 does not use all monotone maps in this definition. The reader can check that using all monotone maps would give the same assembly map, and would allow us to work with simplicial rather than semisimplicial sets in what follows.

${ }^{14}$ To know that this is a correct choice, the paragraph after Observation 1.3 in WW95. says that one simply has to show that it gives an assembly map with the properties in WW95, Theorem 1.1], and this follows from the fact that this choice of $\lambda$ is natural with respect to $X$.
} 
be the functor which takes $f: \Delta^{n} \rightarrow X \times Y$ to the pair $\left(p_{1} \circ f, p_{2} \circ f\right)$, where $p_{1}$ and $p_{2}$ are the projections.

Lemma F.3. (i) The diagram

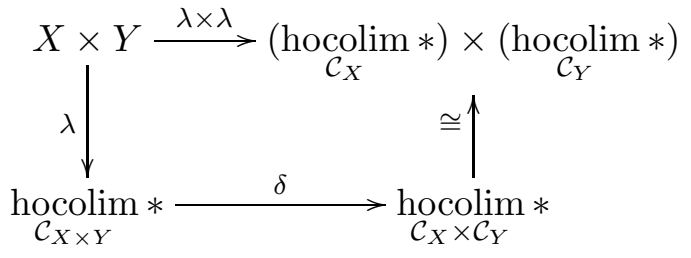

commutes, where $\delta$ is induced by $d$ and the verical arrow is induced by the projections $\mathcal{C}_{X \times Y} \rightarrow \mathcal{C}_{X}$ and $\mathcal{C}_{X \times Y} \rightarrow \mathcal{C}_{Y}$.

(ii) The map $\delta$ in part (i) is a weak equivalence.

The proof of part (i) is left to the reader. Part (ii) follows from (i) and the fact that $\lambda$ is a weak equivalence.

Next we need some notation. For a space $Z$ let $\Lambda$ denote the composite

$$
\left.Z_{+} \wedge \mathbf{F}(*) \stackrel{\lambda \wedge 1}{\longrightarrow} \underset{\mathcal{C}_{Z}}{\operatorname{hocolim}} *\right)_{+} \wedge \mathbf{F}(*) \cong \underset{\mathcal{C}_{Z}}{\operatorname{hocolim}} \mathbf{F}(*)
$$

Let

$$
\delta^{\prime}: \underset{\mathcal{C}_{X \times Y}}{\operatorname{hocolim}} \mathbf{F}(*) \rightarrow \underset{\mathcal{C}_{X} \times \mathcal{C}_{Y}}{\operatorname{hocolim}} \mathbf{F}(*)
$$

be the map induced by $d$; this is a weak equivalence by Lemma F.3(ii). Let $E$ be the functor from $\mathcal{C}_{X} \times \mathcal{C}_{Y}$ to spaces that takes $\left(\Delta^{m} \rightarrow X, \Delta^{n} \rightarrow Y\right)$ to $\Delta^{m} \times \Delta^{n}$, and let

$$
\delta^{\prime \prime}: \underset{\mathcal{C}_{X \times Y}}{\operatorname{hocolim}} \mathbf{F} \circ D \rightarrow \underset{\mathcal{C}_{X} \times \mathcal{C}_{Y}}{\operatorname{hocolim}} \mathbf{F} \circ E
$$

be the map induced by $d$ and the diagonal maps $\Delta^{n} \rightarrow \Delta^{n} \times \Delta^{n}$.

To complete the proof of Proposition F.1, we need only observe that the following diagram is commutative:

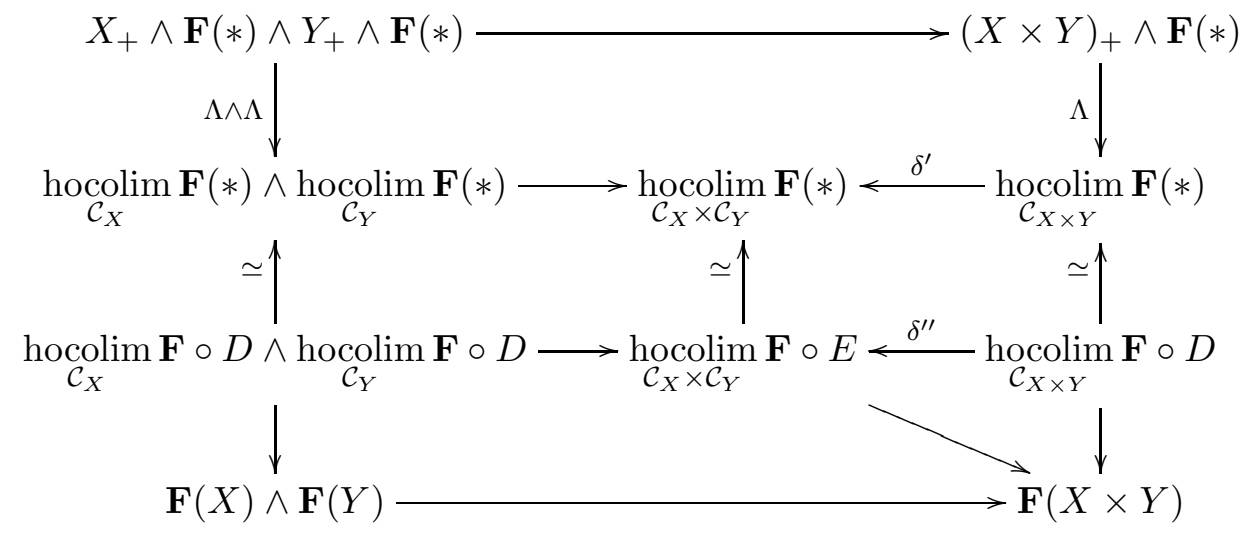




\section{Appendix G. Corrections to some signs in [LM13]}

This appendix is only relevant for the proof of Lemma 13.3. All references are to LM13.

In Definition 15.4(i), the $i$-th face map $\operatorname{ad}^{k}\left(\Delta^{n}\right) \rightarrow \operatorname{ad}^{k}\left(\Delta^{n-1}\right)$ should be $(-1)^{i}$ times the composite with the map induced by the $i$-th coface map $\Delta^{n-1} \rightarrow \Delta^{n}$. There should also be an analogous sign in Definition 17.2.

In order for Proposition 17.8 to be true with this sign, the paragraph that comes after Lemma 15.7 needs to be replaced by the following.

"Next observe that for each $n$ there is an isomorphism of $\mathbb{Z}$-graded categories

$$
\nu: \mathcal{C} \operatorname{ell}\left(\Delta^{n+1}, \partial_{0} \Delta^{n+1} \cup\{0\}\right) \rightarrow \mathcal{C} \operatorname{ell}\left(\partial_{0} \Delta^{n+1}\right)
$$

which lowers degrees by 1 , defined as follows: a simplex $\sigma$ of $\Delta^{n+1}$ which is not in $\partial_{0} \Delta^{n+1} \cup\{0\}$ contains the vertex 0 . Let $\nu$ take $\sigma$ (with its canonical orientation) to the simplex of $\partial_{0} \Delta^{n}$ spanned by the vertices of $\sigma$ other than 0 (with its canonical orientation). Let

$$
\theta: \mathcal{C} \operatorname{ell}\left(\Delta^{n+1}, \partial_{0} \Delta^{n+1} \cup\{0\}\right) \rightarrow \mathcal{C} \operatorname{ell}\left(\Delta^{n}\right)
$$

be the composite of $\eta$ with the isomorphism induced by the face map $\Delta^{n} \rightarrow$ $\partial_{0} \Delta^{n+1}$. $\theta$ is incidence-compatible, so by part (e) of Definition 3.10 it induces a bijection

$$
\theta^{*}: \operatorname{ad}^{k}\left(\Delta^{n}\right) \rightarrow \operatorname{ad}^{k+1}\left(\Delta^{n+1}, \partial_{0} \Delta^{n+1} \cup\{0\}\right) . "
$$

This change leads to corresponding changes in Section 16 and Lemma 17.11, which we leave to the interested reader.

\section{REFERENCES}

[Ada74] J. F. Adams, Stable Homotopy and Generalised Homology, Chicago Lectures in Mathematics, The University of Chicago Press, 1974.

[ABLMP13] P. Albin, M. Banagl, E. Leichtnam, R. Mazzeo, P. Piazza, Refined intersection homology on non-Witt spaces, arXiv:1308.3725.

[ALMP12] P. Albin, E. Leichtnam, R. Mazzeo, P. Piazza, The signature package on Witt spaces, Ann. Sci. École. Norm. Supérieure. (4) 45 (2012), 241 - 310.

[ALMP13] - The Novikov conjecture on Cheeger spaces, arXiv:1308.2844.

[BL16] N. Baas, G. Laures, Singularities and Quinn spectra, appears in Münster J. of Math., arXiv:1404.5395.

[Ban02] M. Banagl, Extending intersection homology type invariants to non-Witt spaces, Memoirs Amer. Math. Soc. 160 (2002), no. 760, 1 - 83.

[Ban07] Topological invariants of stratified spaces, Springer Monographs in Mathematics, Springer-Verlag Berlin Heidelberg, 2007.

[Ban11] _ The Signature of Singular Spaces and its Refinements to Generalized Homology Theories, in: Topology of Stratified Spaces (Friedman, Hunsicker, Libgober, Maxim, eds.), Mathematical Sciences Research Institute Publications 58, Cambridge University Press, New York, 2011, 223 - 249.

[BCS03] M. Banagl, S. E. Cappell, and J. L. Shaneson, Computing twisted signatures and L-classes of stratified spaces, Math. Ann. 326 (2003), no. 3, 589 - 623.

[BM12] A. Blumberg and M. Mandel, Localization theorems in topological Hochschild homology and topological cyclic homology, Geometry and Topology 16 (2012), 1053-1120. 
[Bo84] A. Borel et al., Intersection cohomology, Progr. Math., no. 50, Birkhäuser Verlag, Boston, 1984.

[CSW91] S. E. Cappell, J. L. Shaneson, and S. Weinberger, Classes topologiques caractéristiques pour les actions de groupes sur les espaces singuliers, C. R. Acad. Sci. Paris Sér. I Math. 313 (1991), 293-295.

[CGJ92] D. C. Cohen, M. Goresky, and L. Ji, On the Künneth formula for intersection cohomology, Trans. Amer. Math. Soc. 333 (1992), 63-69.

[C79] P. Conner, Differentiable periodic maps, Second edition, Lecture Notes in Mathematics, 738, Springer Verlag, 1979.

[Dav00] J. Davis, Manifold aspects of the Novikov conjecture, in: Surveys on Surgery Theory, volume 1 (S. Cappell, A. Ranicki, J. Rosenberg, eds.), Annals of Math. Studies 145, Princeton University Press, 2000, 195 - 224.

[DK01] J. Davis and P. Kirk, Lecture Notes in Algebraic Topology, Graduate Studies in Mathematics, volume 35, American Mathematical Society, Providence, 2001.

[Epp07] T. Eppelmann, Signature homology and symmetric L-theory, Ph.D. thesis, Ruprecht-Karls Universität Heidelberg, 2007.

[F03] Stratified fibrations and the intersection homology of the regular neighborhoods of bottom strata, Topology and its Applications 134 (2003), $69-109$.

[F07] G. Friedman, Singular chain intersection homology for traditional and superperversities. Trans. Amer. Math. Soc. 359 (2007), 1977-2019.

[F] Singular intersection homology, in preparation.

[Fa] $\quad$ Stratified and unstratified bordism of pseudomanifolds, in preparation.

[FM13a] G. Friedman, J. McClure, Cup and cap products in intersection (co)homology, Advances in Mathematics 240 (2013), 383-426.

[FM13b] The symmetric signature of a Witt space, Journal of Topology and Analysis 5 (2013), 121-159.

[GM80] M. Goresky and R. D. MacPherson, Intersection homology theory, Topology 19 (1980), 135 - 162.

[GM83] ㄴ. Intersection homology II, Invent. Math. 71 (1983), 77 - 129.

[GS83] R. M. Goresky and P. H. Siegel, Linking Pairings on Singular Spaces, Comm. Math. Helv. 58 (1983), 96 - 110.

[HS71] P. Hilton and U. Stammbach, A course in homological algebra, Graduate Texts in Mathematics, Volume 4, Springer-Verlag. 1971.

[H03] P. Hirschhorn, Model categories and their localizations, Mathematical Surveys and Monographs, Volume 99, American Mathematical Society, 2003.

[HSS00] M. Hovey, B. Shipley, J. Smith, Symmetric spectra, JAMS 13 (2000), 149208.

[KW06] F. Kirwan and J. Woolf, An introduction to intersection homology theory, second ed., Chapman \& Hall/CRC, 2006.

[KMM13] P. Kühl, T. Macko, A. Mole, The total surgery obstruction revisited Münster J. Math. 6 (2013), 181-269.

[LM13] G. Laures and J. E. McClure, Multiplicative properties of Quinn spectra, Forum Math. 264 (2014), 1117-1185.

[LM] Commutativity properties of Quinn spectra, http://front.math.ucdavis.edu/1304.4759

[Lück] W. Lück, Transformation groups and algebraic K-theory, Lecture Notes in Mathematics, 1408, Springer Verlag, 1989.

[M71] A. S. Miščenko, Homotopy invariants of non-simply connected manifolds. III. Higher signatures, Izv. Akad. Nauk SSSR, Ser. mat. 35 (1971), 1316 - 1355. 
[MMSS01] M. Mandell, J. P. May, S. Schwede and B. Shipley, Model categories of diagram spectra, Proc. London Math. Soc. (3) 82 (2001), 441 - 512.

[May67] Simplicial objects in algebraic topology Reprint of the 1967 original. Chicago Lectures in Mathematics. University of Chicago Press, Chicago, IL, 1992.

[Mu84] J. Munkres, Elements of Algebraic Topology, Perseus Book Publishing, 1984.

[Par90] W. L. Pardon, Intersection homology, Poincaré spaces and the characteristic variety theorem, Comment. Math. Helv. 65 (1990), 198 - 233.

[Q95] F. Quinn, Assembly maps in bordism-type theories, in: Novikov Conjectures, Index Theorems and Rigidity (Oberwolfach, 1993), volume 1, London Mathematical Society Lecture Series 226, Cambridge University Press, 1995.

[Ran80] A. A. Ranicki, The algebraic theory of surgery, I. Foundations, Proc. Lond. Math. Soc. 40 (1980), 87 - 192.

[Ran92] _ Algebraic L-theory and topological manifolds, Cambridge Tracts in Math., no. 102, Cambridge University Press, 1992.

[Rezk13] C. Rezk, Frobenius pairs and Atiyah duality, arXiv:1303.3567.

[RS71] C. P. Rourke and B. J. Sanderson, On $\Delta$-sets I, Quart. J. Math. Oxford (2), 22 (1971), 321-338.

[RS72] _ Introduction to piecewise-linear topology, Ergebnisse der Mathematik und ihrer Grenzgebiete, no. 69, Springer Verlag, 1972.

[Rud98] Yu. B. Rudyak, On Thom spectra, orientability, and cobordism, Springer Monographs in Mathematics, Springer-Verlag Berlin Heidelberg, 1998.

[Sch08] S. Schwede, On the homotopy groups of symmetric spectra, Geometry \& Topology 12 (2008), 1313-1344.

[Sch] An untitled book project about symmetric spectra, version 2.4, July 12, 2007, available at http://www.math.uni-bonn.de/ schwede/SymSpec.pdf

[Sie83] P. H. Siegel, Witt spaces: A geometric cycle theory for KO-homology at odd primes, Amer. J. Math. 105 (1983), 1067-1105.

[St68] R. Stong, Notes on Cobordism Theory, Princeton University Press, 1968.

[Weib94] C. Weibel, An introduction to homological algebra, Cambridge studies in advanced mathematics 38, Cambridge University Press, 1994

[W94] S. Weinberger, The topological classification of stratified spaces, Chicago Lecture Notes in Mathematics, University of Chicago Press, 1994.

[WW89] M. Weiss and B. Williams, Automorphisms of manifolds and algebraic Ktheory. II, J. Pure Appl. Algebra 62 (1989), 47 - 107.

[WW95] Assembly, in: Novikov Conjectures, Index Theorems and Rigidity (Oberwolfach, 1993), volume 2, London Mathematical Society Lecture Series 226, Cambridge University Press, 1995.

[Wh78] G. Whitehead, Elements of Homotopy Theory, Graduate Texts in Math., no. 61, Springer-Verlag, 1978.

Mathematisches Institut, Universität Heidelberg, INF 288, D-69120 HeidelBERG, GERMANY

E-mail address: banagl@mathi.uni-heidelberg.de

FAkUltät für Mathematik, Ruhr-Universität Bochum, NA1/66, D-44780 Bochum, Germany

E-mail address: gerd@laures.de

Department of Mathematics, Purdue University, 150 N. University Street, WEST LAFAYETTE, IN 47907-2067, USA

E-mail address: mcclure@math.purdue.edu 